Supporting Information for

\title{
De novo assembly of the benzenoid ring as a core strategy for synthesis of the isoindolinone natural products isohericerin, erinacerin $\mathrm{A}$, and sterenin $\mathrm{A}$
}

\author{
Chenlong Zhu, Juntian Zhang, and Thomas R. Hoye* \\ Department of Chemistry, 207 Pleasant St. SE, University of Minnesota, Minneapolis, MN 55455 \\ email: hoye@umn.edu
}

\section{Table of Contents}

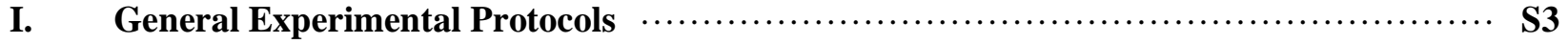

II. Experimental Procedures and Characterization Data for New Compounds $\cdots \ldots \ldots \ldots$ S4-S48

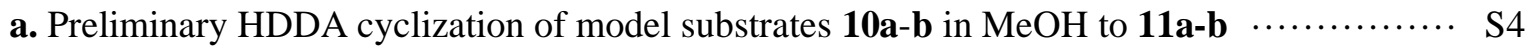

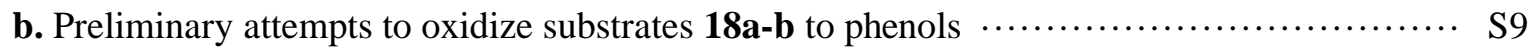

c. Experimental details for preparation and characterization data for S8-S13 and 13-3 $\ldots \ldots \ldots$ S20

III. Tabulated NMR Spectral Data for Comparison of Reported vs. from This Work $\cdots$ S49-S51

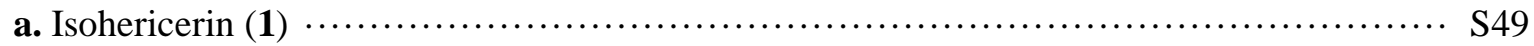

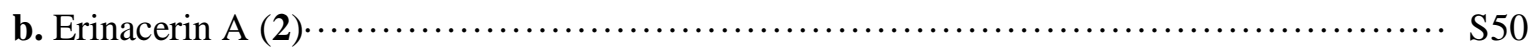

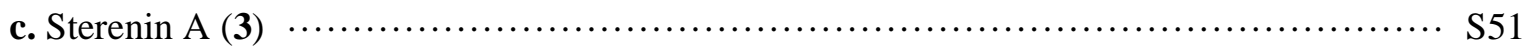

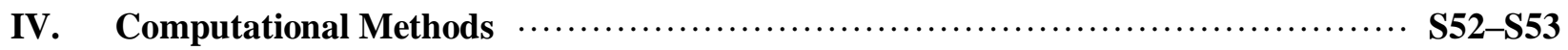

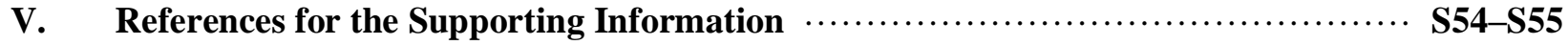

Citations to all 20 examples $^{1}$ of cyclizations of HDDA substrates having amide linkers with the carbonyl group attached to the diynophile rather than the 1,3-diyne, as here. $\cdots . . .$. S5 54

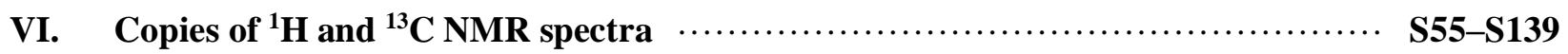

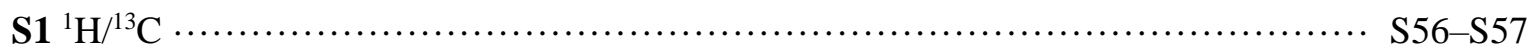

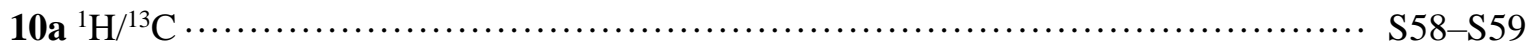

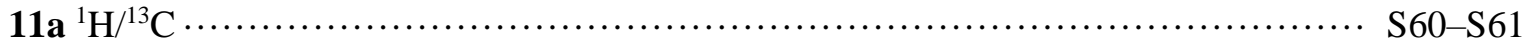

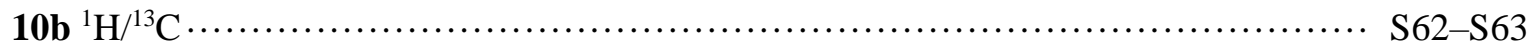

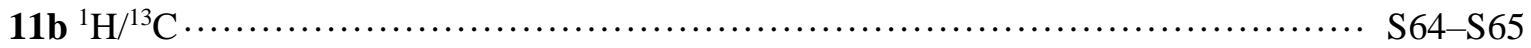

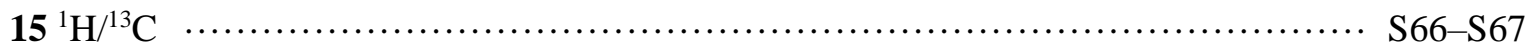

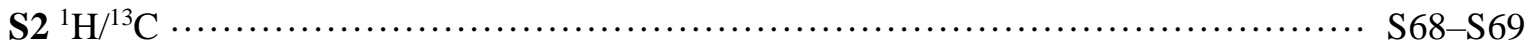




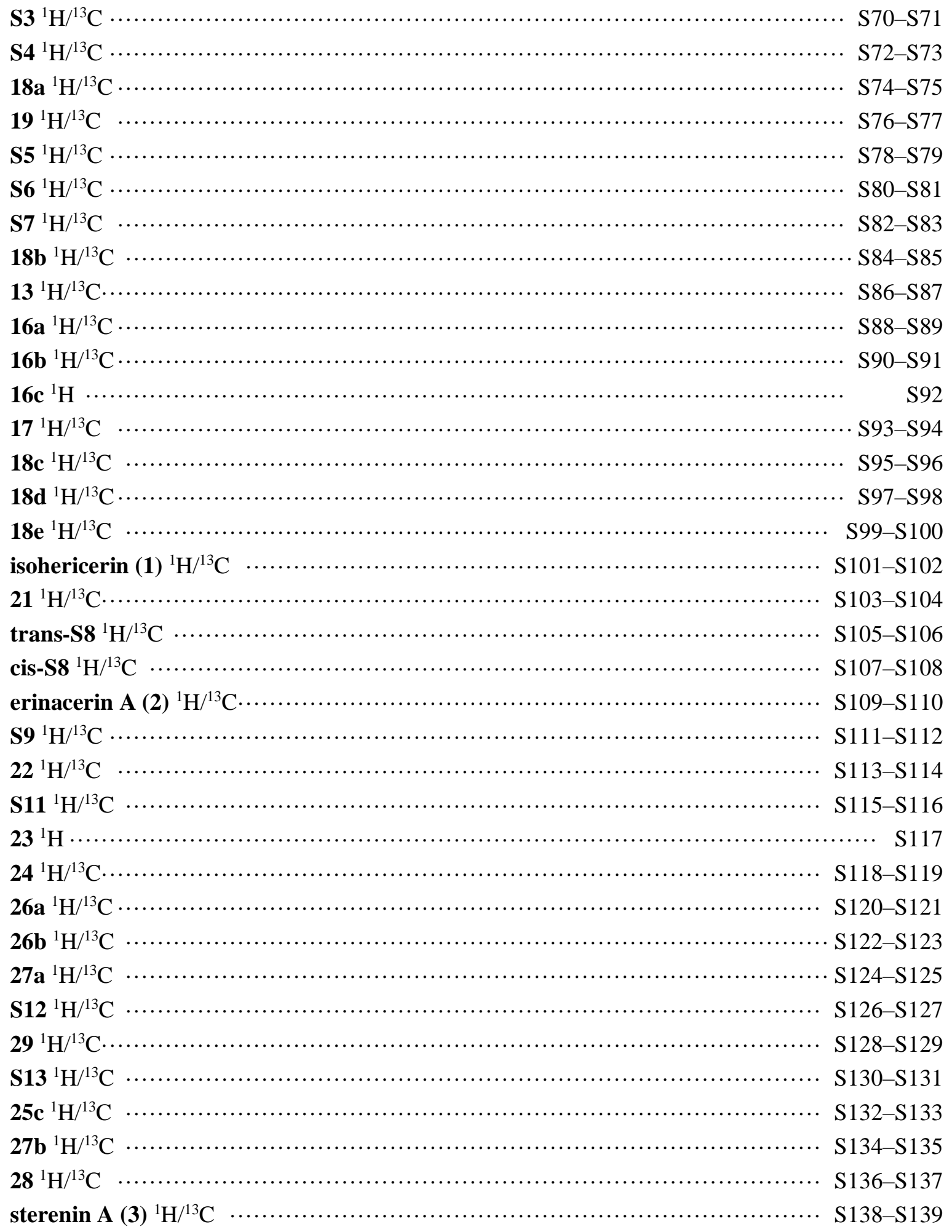




\section{General Experimental Protocols:}

Nuclear magnetic resonance (NMR) spectra $\left({ }^{1} \mathrm{H}\right.$ and $\left.{ }^{13} \mathrm{C}\right)$ were recorded using either a Bruker AX-400 or AX-500 spectrometer. Chemical shifts in ${ }^{1} \mathrm{H}$ NMR spectra recorded in $\mathrm{CDCl}_{3}$ are referenced to TMS $(\delta 0.00)$ or $\mathrm{CD}_{3} \mathrm{OD}(\delta 3.31)$. Data are reported according to the following format: chemical shift (ppm) [multiplicity (e.g., s, d, t, q, etc.), coupling constant(s) (in Hz), integral value (to the nearest whole integer), and structural assignment of the proton]. Coupling constant values have been determined using reported methods. ${ }^{2,3}$ Non-first order triplets in a ${ }^{1} \mathrm{H}$ NMR spectrum (e.g., the chemically equivalent methylene protons in $\mathrm{PhCH}_{a} \mathrm{H}_{\mathrm{a}}{ } \mathrm{CH}_{\mathrm{b}} \mathrm{H}_{\mathrm{b}} \mathrm{NR}_{2}$ ) are designated by 'nfot' and the apparent triplet coupling constant is indicated as $J_{a p p}$. Non-first order multiplets in a ${ }^{1} \mathrm{H}$ NMR spectrum are designated by 'nfom'. Chemical shifts in ${ }^{13} \mathrm{C}$ NMR spectra are referenced to the carbon atom of $\mathrm{CDCl}_{3}(\delta 77.16)$ or $\mathrm{CD}_{3} \mathrm{OD}(\delta 49.15)$. Non-first order doublets or "triplets" in a ${ }^{1} \mathrm{H}$ NMR spectrum (e.g., present in the ortho or meta positions, respectively of monosubstituted benzene ring) are designated by 'nfod' or 'nfot' and the apparent coupling constant is indicated as $J_{a p p}$.

For many of the compounds containing a trisubstituted alkene with two methyl groups (viz. i), the two were distinguished by labeling as $\mathrm{E}$ or $\mathrm{Z}$ according to the convention shown in i. For example, $\mathrm{C}_{12} \mathrm{ECH}_{3}$ refers to the resonance of the methyl group attached to $\mathrm{C} 12$ that is cis to the substituent at $\mathrm{C} 11$ and trans to the hydrogen atom at $\mathrm{C} 11$. In most instances, the chemical shift of the $\mathrm{ECH}_{3}$ protons is further downfield than those of the $\mathrm{ZCH}_{3}$ protons

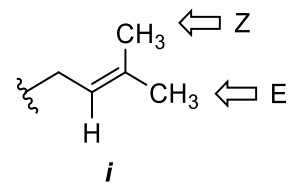
(by $0.05-0.1 \mathrm{ppm}$ ).

Infrared (IR) spectra were measured on a Bruker model Alpha II IR spectrometer. The samples were placed on a diamond window as thin films (solids by evaporation from a $\mathrm{CH}_{2} \mathrm{Cl}_{2}$ solution and liquids by direct deposition) and recorded in the attenuated total reflectance (ATR) mode. The absorption peak maxima are given in $\mathrm{cm}^{-1}$.

High-resolution mass spectrometry (HRMS) measurements were performed in the ESI mode using a Thermo Orbitrap Velos instrument or in CI mode $\left(\mathrm{NH}_{3}\right.$ gas) using an Agilent 7200 GC/QTOF-MS instrument. Pierce ${ }^{T M}$ LTQ or perfluorotributylamine was used as external calibrants. The samples were either directly injected as a solution of the analyte in acetonitrile and carried directly into the ESI source with an elution solvent of 50:50 AN/ $\mathrm{H}_{2} \mathrm{O}$ containing $0.1 \%$ formic acid in the former or injected as an ethyl acetate solution onto a 60 m DB-5 GC column.

Reactions performed above the ambient laboratory temperature were heated in silicone oil baths that had been pre-equilibrated to the temperature of choice before the reaction vessel was immersed. When used as a reaction solvent, chloroform was of a source that did not have ethanol present as a stabilizer. 


\section{Experimental Procedures and Characterization Data for New Compounds}

\section{IIa. Preliminary HDDA cyclization of model substrates 10a-b in MeOH to 11a-b}

\section{$N$-Butylhexa-2,4-diynamide (S1)}

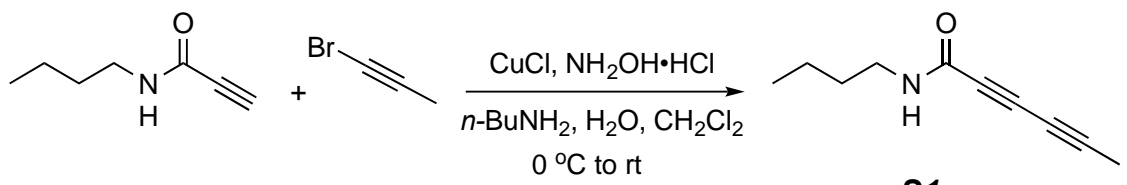

S1

To a stirred solution of $40 \% n-\mathrm{BuNH}_{2} / \mathrm{H}_{2} \mathrm{O}(5 \mathrm{~mL})$ and $\mathrm{CH}_{2} \mathrm{Cl}_{2}(3 \mathrm{~mL})$ was added cuprous chloride (20 mg, $0.2 \mathrm{mmol})$ and hydroxylamine hydrochloride $(128 \mathrm{mg}, 2.0 \mathrm{mmol})$ at $0{ }^{\circ} \mathrm{C}$. In the meantime, a solution of $N$-butylpropiolamide $(250 \mathrm{mg}, 2.0 \mathrm{mmol})$ in $\mathrm{CH}_{2} \mathrm{Cl}_{2}(2 \mathrm{~mL})$ was added in a dropwise fashion into the reaction vial. The mixture was stirred at $0{ }^{\circ} \mathrm{C}$ for $5 \mathrm{~min}$, and a solution of 1bromoprop-1-yne (1.1 M in hexane, $2 \mathrm{~mL}, 2.2 \mathrm{mmol})$ in $\mathrm{CH}_{2} \mathrm{Cl}_{2}(2 \mathrm{~mL})$ was added slowly (1 drop per 2 seconds). After the addition, the reaction mixture was allowed to warm to ambient temperature for $30 \mathrm{~min}$ before it was quenched by the addition of $\mathrm{NH}_{4} \mathrm{Cl}(10 \mathrm{~mL}$, satd aq). The layers were separated and the aqueous layer was extracted with $\mathrm{CH}_{2} \mathrm{Cl}_{2}(3 \times 10 \mathrm{~mL})$. The combined organic layers were washed with brine $(20 \mathrm{~mL})$, dried $\left(\mathrm{Na}_{2} \mathrm{SO}_{4}\right)$, and concentrated in vacuo. Flash column chromatography (silica gel, hexanes:EtOAc 2:1) afforded the diyne S1 (275 mg, 85\%) as a light yellow oil.

Two rotamers for this secondary amide (ca. 7:1) were evident in the NMR spectral data:

${ }^{1} \mathbf{H}$ NMR $\left(500 \mathrm{MHz}, \mathrm{CDCl}_{3}\right): \delta 5.96\left(\right.$ br s, $\left.1 \mathrm{H}, \mathrm{CH}_{2} \mathrm{NH}\right), 3.40\left(\mathrm{nfot}, J_{\text {app }}=6.9 \mathrm{~Hz}, 0.25 \mathrm{H}\right.$, $\mathrm{NCH}_{2} \mathrm{CH}_{2}$ ), 3.49 (nfot, $J_{\text {app }}=7.0 \mathrm{~Hz}, 1.75 \mathrm{H}, \mathrm{NCH}_{2} \mathrm{CH}_{2}$ ), $2.03(\mathrm{~s}, 0.38 \mathrm{H}, \mathrm{C} \equiv \mathrm{CCH}$ ), $2.00(\mathrm{~s}, 2.62 \mathrm{H}$, $\mathrm{C} \equiv \mathrm{CCH}_{3}$ ), $1.53-1.47$ (apparent pentet, $J=7.5 \mathrm{~Hz}, 2 \mathrm{H}, \mathrm{NCH}_{2} \mathrm{CH}_{2} \mathrm{CH}_{2} \mathrm{CH}_{3}$ ), 1.40-1.31 (apparent sextet, $J=7.5 \mathrm{~Hz}, 2 \mathrm{H}, \mathrm{NCH}_{2} \mathrm{CH}_{2} \mathrm{CH}_{2} \mathrm{CH}_{3}$ ), and $0.96-0.91$ (two overlapping triplets, $J=7.3 \mathrm{~Hz}$, $3 \mathrm{H}, \mathrm{NCH}_{2} \mathrm{CH}_{2} \mathrm{CH}_{2} \mathrm{CH}_{3}$ ).

${ }^{13} \mathrm{C}$ NMR $\left(125 \mathrm{MHz}, \mathrm{CDCl}_{3}\right): \delta$ 155.2,* 152.4, 83.8,* 82.1, 70.2, 67.6, 63.12,*63.10, 43.1,* 39.9, $32.7, * 31.4,20.1,19.8 *{ }^{*} 13.77,13.75, *$ and $4.8 *{ }^{*} 4.7$.

*from minor rotamer (two not definitively identified)

HRMS (ESI) $m / z:[\mathrm{M}+\mathrm{H}]^{+}$Calcd for $\mathrm{C}_{10} \mathrm{H}_{14} \mathrm{NO}^{+}$164.1070; Found 164.1064.

IR (neat): 3265, 2962, 2932, 2870, 2247, and $1627 \mathrm{~cm}^{-1}$. 


\section{$N$-Butyl- $N$-(prop-2-yn-1-yl)hexa-2,4-diynamide (10a)}

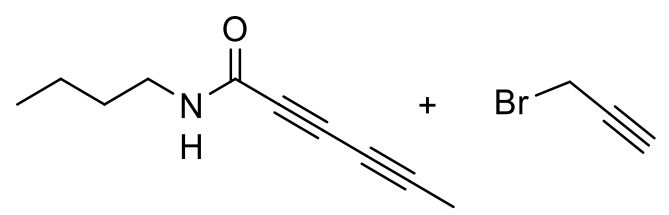

S1

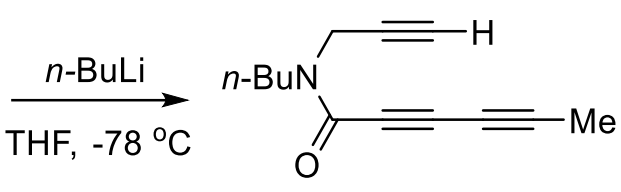

$10 a$

To a stirred solution of amide S1 $(250 \mathrm{mg}, 1.5 \mathrm{mmol})$ in THF $(5 \mathrm{~mL})$ was added a solution of $n$ $\operatorname{BuLi}(2.5 \mathrm{M}$ in hexanes, $0.55 \mathrm{~mL}, 0.9 \mathrm{mmol})$ dropwise at $-78^{\circ} \mathrm{C}$. The resulting solution was stirred for an additional $30 \mathrm{~min}$, followed by the dropwise addition of propargyl bromide ( $80 \mathrm{wt} . \%$ in toluene, $170 \mu \mathrm{L}, 1.5 \mathrm{mmol}$ ) at $-78{ }^{\circ} \mathrm{C}$. The reaction mixture was allowed to warm to ambient temperature and further stirred for $1 \mathrm{~h}$. The light yellow slurry was quenched with satd $\mathrm{NH}_{4} \mathrm{Cl}$ solution, extracted with hexanes $(3 \times 10 \mathrm{~mL})$, and washed with brine $(20 \mathrm{~mL})$. The combined organic layer was dried and concentrated. The residue was purified by flash chromatography (hexanes:EtOAc 2:1) to give the amide 10a as a light yellow oil (250 mg, 90\%).

Two rotamers for the tertiary amide (ca. 3:2) were evident in the NMR spectral data:

${ }^{1} \mathbf{H}$ NMR $\left(500 \mathrm{MHz}, \mathrm{CDCl}_{3}\right): \delta 4.34\left(\mathrm{~d}, J=2.5 \mathrm{~Hz}, 0.8 \mathrm{H}, \mathrm{C} \equiv \mathrm{CCH}_{2} \mathrm{~N}\right.$, minor), $4.22(\mathrm{~d}, J=1.1 \mathrm{~Hz}$, 1.2H, $\mathrm{C} \equiv \mathrm{CCH}_{2} \mathrm{~N}$, major), 3.67 (nfot, $J_{\text {app }}=7.4 \mathrm{~Hz}, 1.2 \mathrm{H}, \mathrm{NCH}_{2} \mathrm{CH}_{2}$ ), 3.49 (nfot, $J_{\text {app }}=7.7 \mathrm{~Hz}$, $\left.0.8 \mathrm{H}, \mathrm{NCH}_{2} \mathrm{CH}_{2}\right), 2.32(\mathrm{t}, J=2.5 \mathrm{~Hz}, 0.4 \mathrm{H}, \mathrm{C} \equiv \mathrm{CH}), 2.22(\mathrm{t}, J=2.5 \mathrm{~Hz}, 0.6 \mathrm{H}, \mathrm{C} \equiv \mathrm{CH}), 2.02(\mathrm{~s}, 3 \mathrm{H}$, $\mathrm{C} \equiv \mathrm{CCH}_{3}$ ), 1.66-1.60 (apparent pentet, $J=7.5 \mathrm{~Hz}, 2 \mathrm{H}, \mathrm{NCH}_{2} \mathrm{CH}_{2} \mathrm{CH}_{2} \mathrm{CH}_{3}$ ), 1.41-1.29 (apparent sextet, $J=7.5 \mathrm{~Hz}, 2 \mathrm{H}, \mathrm{NCH}_{2} \mathrm{CH}_{2} \mathrm{CH}_{2} \mathrm{CH}_{3}$ ), and $0.99-0.90$ (two overlapping triplets, $J=7.3 \mathrm{~Hz}$, $3 \mathrm{H}, \mathrm{NCH}_{2} \mathrm{CH}_{2} \mathrm{CH}_{2} \mathrm{CH}_{3}$ ).

${ }^{13} \mathrm{C}$ NMR $\left(125 \mathrm{MHz}, \mathrm{CDCl}_{3}\right): \delta 153.4,153.2,84.0,83.8,78.04,77.96,76.3,76.2,73.1,72.3,65.5$, $65.4,63.22,63.21,48.2,44.5,38.6,33.6,30.5,29.2,20.2,19.9,13.91,13.86$, and 4.8 .

HRMS (ESI) $m / z:[\mathrm{M}+\mathrm{H}]^{+}$Calcd for $\mathrm{C}_{13} \mathrm{H}_{16} \mathrm{NO}^{+}$202.1226; Found 202.1216.

IR (neat): 3304, 3230, 2960, 2931, 2874, 2246, and $1640 \mathrm{~cm}^{-1}$. 


\section{2-Butyl-6-methoxy-5-methylisoindolin-1-one (11a)}

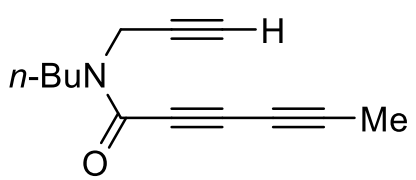

$10 a$
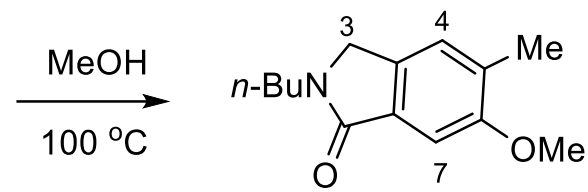

$11 a$

A solution of the amide $10 \mathrm{a}(150 \mathrm{mg}, 0.75 \mathrm{mmol})$ in methanol $(5 \mathrm{~mL})$ was heated in a screwcapped culture tube in an oil bath held at $100{ }^{\circ} \mathrm{C}$. After $4 \mathrm{~h}$ the slightly yellow-colored solution was allowed to cool to room temperature and concentrated in vacuo. Flash column chromatography (silica gel, hexanes:EtOAc 4:1) afforded the lactam 11a $(153 \mathrm{mg}, 88 \%)$ as a light yellow oil.

${ }^{1} \mathbf{H}$ NMR $\left(400 \mathrm{MHz}, \mathrm{CDCl}_{3}\right): \delta 7.26(\mathrm{~s}, 1 \mathrm{H}, \mathrm{C} 7 H), 7.18(\mathrm{~s}, 1 \mathrm{H}, \mathrm{C} 4 H), 4.27(\mathrm{~s}, 2 \mathrm{H}, \mathrm{C} 3 H), 3.89$ (s, $\left.3 \mathrm{H}, \mathrm{OCH}_{3}\right), 3.60$ (t, $\left.J=7.3 \mathrm{~Hz}, 2 \mathrm{H}, \mathrm{NCH}_{2} \mathrm{CH}_{2}\right) 2.29$ (s, $3 \mathrm{H}, \mathrm{ArCH}_{3}$ ), 1.64 (apparent pentet, $J=7.4$ $\mathrm{Hz}, 2 \mathrm{H}, \mathrm{NCH}_{2} \mathrm{CH}_{2} \mathrm{CH}_{2} \mathrm{CH}_{3}$ ), 1.38 (apparent sextet, $J=7.4 \mathrm{~Hz}, 2 \mathrm{H}, \mathrm{NCH}_{2} \mathrm{CH}_{2} \mathrm{CH}_{2} \mathrm{CH}_{3}$ ), and 0.95 (t, $J=7.4 \mathrm{~Hz}, 2 \mathrm{H}, \mathrm{NCH}_{2} \mathrm{CH}_{2} \mathrm{CH}_{2} \mathrm{CH}_{3}$ ).

${ }^{13} \mathrm{C}$ NMR $\left(101 \mathrm{MHz}, \mathrm{CDCl}_{3}\right): \delta 169.0,158.2,133.2,132.1,131.2,124.4,104.2,55.8,49.5,42.3$, $30.7,20.2,17.2$, and 13.9.

HRMS (ESI) $m / z:[\mathrm{M}+\mathrm{H}]^{+}$Calcd for $\mathrm{C}_{14} \mathrm{H}_{20} \mathrm{NO}_{2}{ }^{+}$234.1489; Found 234.1479.

IR (neat): 2962, 2931, 2863, 1680, and $1620 \mathrm{~cm}^{-1}$. 


\section{$N$-Butyl- $N$-(3-(trimethylsilyl)prop-2-yn-1-yl)hexa-2,4-diynamide (10b)}

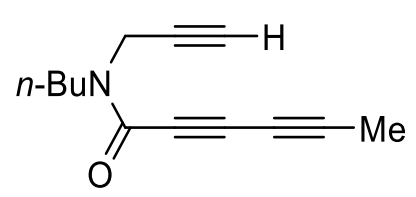

$10 a$

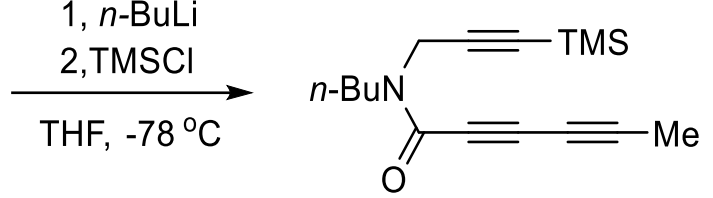

$10 b$

To a stirred solution of amide $10 \mathrm{a}(200 \mathrm{mg}, 1.0 \mathrm{mmol})$ in THF (5 mL) was added a solution of $n$ $\operatorname{BuLi}(2.5 \mathrm{M}$ in hexanes, $0.36 \mathrm{~mL}, 0.9 \mathrm{mmol})$ dropwise at $-78^{\circ} \mathrm{C}$. The resulting lithium acetylide solution was stirred for an additional $30 \mathrm{~min}$, followed by the dropwise addition of TMSCl (126 $\mu \mathrm{L}, 1.0 \mathrm{mmol})$ at $-78{ }^{\circ} \mathrm{C}$. The reaction mixture was allowed to warm to ambient temperature and further stirred for $1 \mathrm{~h}$. The light yellow slurry was quenched with satd $\mathrm{NH}_{4} \mathrm{Cl}$ solution, extracted with hexanes $(3 \times 10 \mathrm{~mL})$, and washed with brine $(20 \mathrm{~mL})$. The combined organic layer was dried and concentrated. The residue was purified by flash chromatography (hexanes:EtOAc 5:1) to give the amide $\mathbf{1 0 b}$ as a light yellow oil (205 $\mathrm{mg}, 84 \%)$.

Two rotamers for the tertiary amide (ca. 3:2) were evident in the NMR spectral data:

${ }^{1} \mathrm{H}$ NMR $\left(500 \mathrm{MHz}, \mathrm{CDCl}_{3}\right): \delta 4.35\left(\mathrm{~s}, 0.8 \mathrm{H}, \mathrm{C} \equiv \mathrm{CCH}_{2} \mathrm{~N}\right.$, minor), $4.24\left(\mathrm{~s}, 1.2 \mathrm{H}, \mathrm{C} \equiv \mathrm{CCH}_{2} \mathrm{~N}\right.$, major), 3.66 (nfot, $J_{\text {app }}=7.3 \mathrm{~Hz}, 1.2 \mathrm{H}, \mathrm{NCH}_{2} \mathrm{CH}_{2}$ ), 3.48 (nfot, $J_{\text {app }}=7.6 \mathrm{~Hz}, 0.8 \mathrm{H}, \mathrm{NCH}_{2} \mathrm{CH}_{2}$ ), 2.02 (s, 3H, C $\equiv \mathrm{CCH}_{3}$ ), 1.63 (apparent pentet, $J=7.5 \mathrm{~Hz}, 1.8 \mathrm{H}, \mathrm{NCH}_{2} \mathrm{CH}_{2} \mathrm{CH}_{2} \mathrm{CH}_{3}$ ), 1.57 (apparent pentet, $J=7.6 \mathrm{~Hz}, 1 \mathrm{H}, \mathrm{NCH}_{2} \mathrm{CH}_{2} \mathrm{CH}_{2} \mathrm{CH}_{3}$ ), 1.37 and 1.32 (overlapping apparent sextets, $J=7.5$ $\mathrm{Hz}, 2 \mathrm{H}, \mathrm{NCH}_{2} \mathrm{CH}_{2} \mathrm{CH}_{2} \mathrm{CH}_{3}$ ), 0.97 (t, $\left.J=7.3 \mathrm{~Hz}, 2.0 \mathrm{H}, \mathrm{NCH}_{2} \mathrm{CH}_{2} \mathrm{CH}_{2} \mathrm{CH}_{3}\right), 0.92$ (t, $J=7.4 \mathrm{~Hz}$, 1.5H, $\left.\mathrm{NCH}_{2} \mathrm{CH}_{2} \mathrm{CH}_{2} \mathrm{CH}_{3}\right), 0.17$ [s, 3.6H, $\mathrm{Si}\left(\mathrm{CH}_{3}\right)_{3}$, minor], and 0.16 [s, 5.4H, $\mathrm{Si}\left(\mathrm{CH}_{3}\right)_{3}$, major].

${ }^{13} \mathrm{C}$ NMR $\left(125 \mathrm{MHz}, \mathrm{CDCl}_{3}\right): \delta 153.3,153.1,99.51,99.48,90.3,89.5,83.8,83.6,76.3,76.0,65.7$, 65.6, 63.3, 63.2, 48.1, 44.5, 39.7, 34.5, 30.6, 29.2, 20.2, 19.9, 13.93, 13.89, 4.823, 4.812, -0.09, and -0.13 .

HRMS (ESI) $m / z$ : $[\mathrm{M}+\mathrm{H}]^{+}$Calcd for $\mathrm{C}_{16} \mathrm{H}_{24} \mathrm{NOSi}^{+} 274.1622$; Found 274.1612.

IR (neat): 2959, 2932, 2873, 2245, and $1632 \mathrm{~cm}^{-1}$. 


\section{2-Butyl-6-methoxy-5-methyl-4-(trimethylsilyl)isoindolin-1-one (11b)}

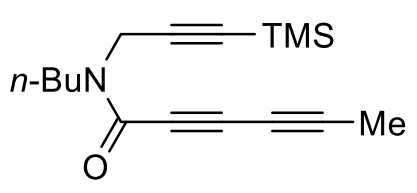

$10 b$

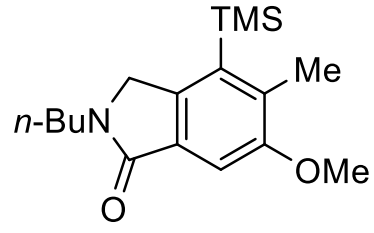

$11 b$

A solution of the amide $10 \mathrm{~b}(102 \mathrm{mg}, 0.37 \mathrm{mmol})$ in methanol $(5 \mathrm{~mL})$ was heated in a screwcapped culture tube in an oil bath held at $100{ }^{\circ} \mathrm{C}$. After $4 \mathrm{~h}$ the slightly yellow-colored solution was allowed to cool to room temperature and concentrated in vacuo. Flash column chromatography (silica gel, hexanes:EtOAc 4:1) afforded the lactam 11b (85 mg, 75\%) as a light yellow oil.

${ }^{1} \mathbf{H}$ NMR $\left(500 \mathrm{MHz}, \mathrm{CDCl}_{3}\right): \delta 7.31$ (s, 1H, $\left.\mathrm{ArH}\right), 4.32$ (s, 2H, NCH $\left.\mathrm{NH}_{2} \mathrm{Ar}\right), 3.88\left(\mathrm{~s}, 3 \mathrm{H}, \mathrm{OCH}_{3}\right), 3.60$ (t, $\left.J=7.3 \mathrm{~Hz}, 2 \mathrm{H}, \mathrm{NCH}_{2} \mathrm{CH}_{2}\right) 2.39$ (s, 3H, $\mathrm{ArCH}_{3}$ ), 1.64 (apparent pentet, $J=7.4 \mathrm{~Hz}, 2 \mathrm{H}$, $\mathrm{NCH}_{2} \mathrm{CH}_{2} \mathrm{CH}_{2} \mathrm{CH}_{3}$ ), 1.39 (apparent sextet, $J=7.4 \mathrm{~Hz}, 2 \mathrm{H}, \mathrm{NCH}_{2} \mathrm{CH}_{2} \mathrm{CH}_{2} \mathrm{CH}_{3}$ ), 0.96 (t, $J=7.4$ $\left.\mathrm{Hz}, 2 \mathrm{H}, \mathrm{NCH}_{2} \mathrm{CH}_{2} \mathrm{CH}_{2} \mathrm{CH}_{3}\right)$, and 0.41 [s, 9H, $\left.\mathrm{Si}\left(\mathrm{CH}_{3}\right)_{3}\right]$.

${ }^{13}$ C NMR (125 MHz, $\left.\mathrm{CDCl}_{3}\right): \delta 168.7,157.9,138.5,137.0,134.0,131.4,105.3,55.8,52.1,42.2$, $30.8,20.3,17.4,14.0$, and 2.0.

HRMS (ESI) $m / z:[\mathrm{M}+\mathrm{H}]^{+}$Calcd for $\mathrm{C}_{17} \mathrm{H}_{28} \mathrm{NO}_{2} \mathrm{Si}^{+}$306.1884; Found 306.1871.

IR (neat): 2958, 2931, 2878, and $1688(\mathrm{~s}, \mathrm{C}=\mathrm{O}) \mathrm{cm}^{-1}$. 


\section{IIb. Preliminary attempts to oxidize substrates $18 \mathrm{a}-\mathrm{b}$ to phenols}

\section{(E)-5,9-Dimethyldeca-4,8-dien-1-yne (15)}

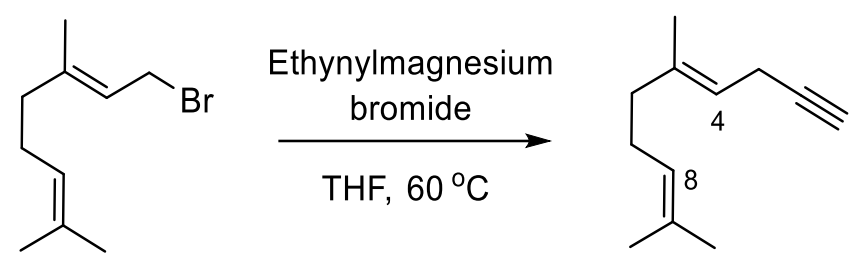

15

To a stirred culture tube containing neat geranyl bromide $(1.09 \mathrm{~g}, 5.0 \mathrm{mmol})$ was added a solution of ethynyl magnesium bromide $(0.5 \mathrm{M}$ in THF, $15 \mathrm{~mL}, 7.5 \mathrm{mmol})$. The reaction mixture was heated overnight in a screw-capped culture tube in an oil bath held at ca. $60{ }^{\circ} \mathrm{C}$. The solution was then quenched with satd $\mathrm{NH}_{4} \mathrm{Cl}$ solution, extracted with hexanes $(3 \times 10 \mathrm{~mL})$, and washed with brine $(20 \mathrm{~mL})$. The combined organic layer was dried and concentrated. The residue was purified by flash chromatography (hexanes:EtOAc 11:1) to give dienyne 15 as a yellow oil (772 mg, 95\%).

${ }^{1} \mathbf{H}$ NMR $\left(500 \mathrm{MHz}, \mathrm{CDCl}_{3}\right): \delta 5.21$ (ttq, $\left.J=6.9,1.4,1.4 \mathrm{~Hz}, 1 \mathrm{H}, \mathrm{C} 4 H\right), 5.09$ (tqq, $J=6.9,1.3$, $1.3 \mathrm{~Hz}, 1 \mathrm{H}, \mathrm{C} 8 H$ ), 2.90 (ddtq, $J=6.9,2.7,0.8,0.8 \mathrm{~Hz}, 2 \mathrm{H}, \mathrm{C} \equiv \mathrm{CCH}_{2} \mathrm{CH}=\mathrm{C}$ ), 2.08 (br dt, $J=7,7$ $\mathrm{Hz}, 2 \mathrm{H}, \mathrm{C} 7 \mathrm{H}_{2}$ ), 2.01 (br t, $J=7 \mathrm{~Hz}, 2 \mathrm{H}, \mathrm{C} 6 \mathrm{H}_{2}$ ), 1.97 (t, $J=2.8 \mathrm{~Hz}, 1 \mathrm{H}, \mathrm{C} \equiv \mathrm{CH}$ ), 1.68 (br d, $J=1.2$

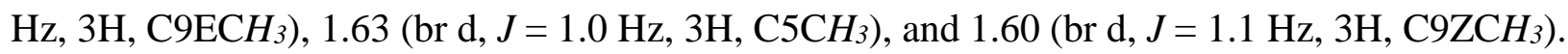

${ }^{13}$ C NMR (125 MHz, $\left.\mathrm{CDCl}_{3}\right): \delta 138.0,131.8,124.1,118.4,83.6,67.7,39.6,26.6,25.9,17.8,17.7$, and 16.3.

HRMS (CI) $m / z:\left[\mathrm{M}+\mathrm{NH}_{4}\right]^{+}$Calcd for $\mathrm{C}_{12} \mathrm{H}_{22} \mathrm{~N}^{+}$180.1747; Found 180.1741.

IR (neat): 3310, 2968, 2917, 2856, and $2729 \mathrm{~cm}^{-1}$. 


\section{(E)-1-Bromo-5,9-dimethyldeca-4,8-dien-1-yne (S2)}

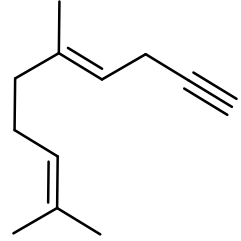

15

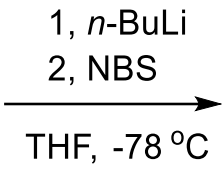

52

To a stirred solution of dienyne $15(650 \mathrm{mg}, 4.0 \mathrm{mmol})$ in THF (5 mL) was added a solution of $n$ $\operatorname{BuLi}(2.5 \mathrm{M}$ in hexanes, $1.68 \mathrm{~mL}, 4.2 \mathrm{mmol})$ dropwise at $-78{ }^{\circ} \mathrm{C}$. The resulting lithium acetylide solution was stirred for an additional $10 \mathrm{~min}$, followed by the dropwise addition of a solution of NBS (854 mg, $4.8 \mathrm{mmol})$ in THF $(8 \mathrm{~mL})$ at $-78^{\circ} \mathrm{C}$. The reaction mixture was allowed to warm to ambient temperature and further stirred for $2 \mathrm{~h}$. The light yellow slurry was quenched with satd $\mathrm{NH}_{4} \mathrm{Cl}$ solution, extracted with hexanes $(3 \times 10 \mathrm{~mL})$, and washed with brine $(20 \mathrm{~mL})$. The combined organic layer was dried and concentrated. The residue was purified by flash chromatography (hexanes:EtOAc 11:1) to give the bromoalkyne $\mathbf{S 2}$ as an orange oil (963 $\mathrm{mg}, 99 \%$ ).

${ }^{1} \mathbf{H}$ NMR $\left(500 \mathrm{MHz}, \mathrm{CDCl}_{3}\right): \delta 5.17(\mathrm{ttq}, J=6.8,1.4,1.4 \mathrm{~Hz}, 1 \mathrm{H}, \mathrm{C} 4 H), 5.08(\mathrm{tqq}, J=6.8,1.3$, $1.3 \mathrm{~Hz}, 1 \mathrm{H}, \mathrm{C} 8 H), 2.91\left(\mathrm{dtq}, J=6.9,0.8,0.8 \mathrm{~Hz}, 2 \mathrm{H}, \mathrm{C} \equiv \mathrm{CCH}_{2} \mathrm{CH}=\mathrm{C}\right), 2.07$ (br dt, $J=7,7 \mathrm{~Hz}$, 2H, C7H2), 2.00 (br t, $\left.J=7 \mathrm{~Hz}, 2 \mathrm{H}, \mathrm{C} 6 \mathrm{H}_{2}\right), 1.68$ (dt, $J=1.3,1.3 \mathrm{~Hz}, 3 \mathrm{H}, \mathrm{C} 9 \mathrm{ECH}$ ), 1.62 (dt, $J=$ $1.4,0.9 \mathrm{~Hz}, 3 \mathrm{H}, \mathrm{C}_{5} \mathrm{CH}_{3}$ ), and 1.60 (br d, $J=1.0 \mathrm{~Hz}, 3 \mathrm{H}, \mathrm{C}_{2} \mathrm{ZCH}_{3}$ ).

${ }^{13} \mathrm{C}$ NMR (125 MHz, $\left.\mathrm{CDCl}_{3}\right): \delta 138.2,131.9,124.1,117.9,79.2,39.5,26.6,25.8,18.9,17.8$, and 16.3. (bromine-bearing alkyne carbon not identified)

HRMS (CI) $m / z$ : $\left[\mathrm{M}+\mathrm{NH}_{4}\right]^{+}$Calculated for $\mathrm{C}_{12} \mathrm{H}_{21}{ }^{79} \mathrm{BrN}^{+} 258.0852$; Found 258.0852.

IR (neat): 2961, 2928, 2855, and $2174 \mathrm{~cm}^{-1}$. 


\section{$N$-(3-(Benzyldimethylsilyl)prop-2-yn-1-yl)-N-phenethylpropiolamide (S3)}

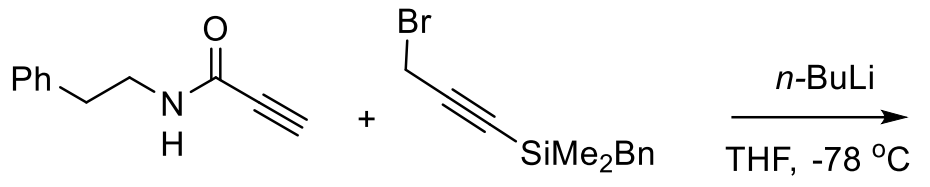

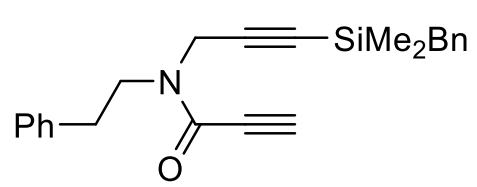

S3

To a stirred solution of $N$-phenethylpropiolamide $(150 \mathrm{mg}, 0.86 \mathrm{mmol})$ in THF $(4 \mathrm{~mL})$ was added a solution of $n$-BuLi $(2.5 \mathrm{M}$ in hexanes, $0.35 \mathrm{~mL}, 0.86 \mathrm{mmol})$ dropwise at $-78{ }^{\circ} \mathrm{C}$. The resulting solution was stirred for an additional $30 \mathrm{~min}$, followed by the dropwise addition of benzyl(3bromoprop-1-yn-1-yl)dimethylsilane ${ }^{4}(230 \mathrm{mg}, 0.86 \mathrm{mmol})$ at $-78^{\circ} \mathrm{C}$. The reaction mixture was allowed to warm to ambient temperature and further stirred for $1 \mathrm{~h}$. The light yellow slurry was quenched with satd $\mathrm{NH}_{4} \mathrm{Cl}$ solution, extracted with hexanes $(3 \times 10 \mathrm{~mL})$, and washed with brine (20 $\mathrm{mL}$ ). The combined organic layer was dried and concentrated. The residue was purified by flash chromatography (hexanes:EtOAc 2:1) to give the amide $\mathbf{S 3}$ as a light yellow oil (230 mg, 76\%).

Two rotamers for the tertiary amide (ca. 3:2) were evident in the NMR spectral data:

${ }^{1} \mathbf{H}$ NMR $\left(500 \mathrm{MHz}, \mathrm{CDCl}_{3}\right): \delta 7.33-7.28(\mathrm{~m}, 2 \mathrm{H}, \mathrm{PhH}), 7.27-7.16(\mathrm{~m}, 5 \mathrm{H}, \mathrm{PhH}), 7.10-7.03(\mathrm{~m}$, $3 \mathrm{H}, \mathrm{PhH}), 4.26\left(\mathrm{~s}, 0.8 \mathrm{H}, \mathrm{C} \equiv \mathrm{CCH}_{2} \mathrm{~N}\right.$, minor), $4.25\left(\mathrm{~s}, 1.2 \mathrm{H}, \mathrm{C} \equiv \mathrm{CCH}_{2} \mathrm{~N}\right.$, major), 3.87 (nfot, $J=7.8$ $\mathrm{Hz}, 1.2 \mathrm{H}, \mathrm{NCH}_{2} \mathrm{CH}_{2}$, major), 3.68 (nfot, $J=7.8 \mathrm{~Hz}, 0.8 \mathrm{H}, \mathrm{NCH}_{2} \mathrm{CH}_{2}$, minor), 3.13 (s, $0.4 \mathrm{H}$, $\mathrm{C} \equiv \mathrm{CH}$, minor), 3.09 (s, 0.6H, $\mathrm{C} \equiv \mathrm{CH}$, major), 2.94 (nfot, $J=7.9 \mathrm{~Hz}, 1.2 \mathrm{H}, \mathrm{NCH}_{2} \mathrm{CH}_{2}$, major), 2.89 (nfot, $J=7.6 \mathrm{~Hz}, 0.8 \mathrm{H}, \mathrm{NCH}_{2} \mathrm{CH}_{2}$, minor), 2.20 (s, 1.2H, $\mathrm{PhCH}_{2} \mathrm{Si}$ major), 2.19 (s, 0.8H, $\mathrm{PhCH} 2 \mathrm{Si}$, minor), 0.142 [s, 2.4H, $\mathrm{Si}\left(\mathrm{CH}_{3}\right)_{2}$, minor], and 0.140 [s, 3.6H, $\mathrm{Si}\left(\mathrm{CH}_{3}\right)_{2}$, major].

${ }^{13} \mathrm{C}$ NMR (125 MHz, $\left.\mathrm{CDCl}_{3}\right): \delta 153.0,152.9,138.8,138.7,138.6,138.0,128.92,128.77,128.8$, $128.5,128.44,128.38,128.38,128.36,126.9,126.7,124.7,124.6,100.73,100.65,88.9,88.5,79.4$, $78.9,77.5,75.6,49.8,46.5,40.3,35.3,34.9,33.5,26.15,26.10,-2.07$ and -2.09 .

HRMS (ESI) $m / z:[\mathrm{M}+\mathrm{H}]^{+}$Calculated for $\mathrm{C}_{23} \mathrm{H}_{26} \mathrm{NOSi}^{+} 360.1778$; Found 360.1764.

IR (neat): 2957, 2928, 1738, and $1646 \mathrm{~cm}^{-1}$. 


\section{(E)- $N$-(3-(Benzyldimethylsilyl)prop-2-yn-1-yl)-8,12-dimethyl- $N$-phenethyltrideca-7,11-dien- 2,4-diynamide (S4)}

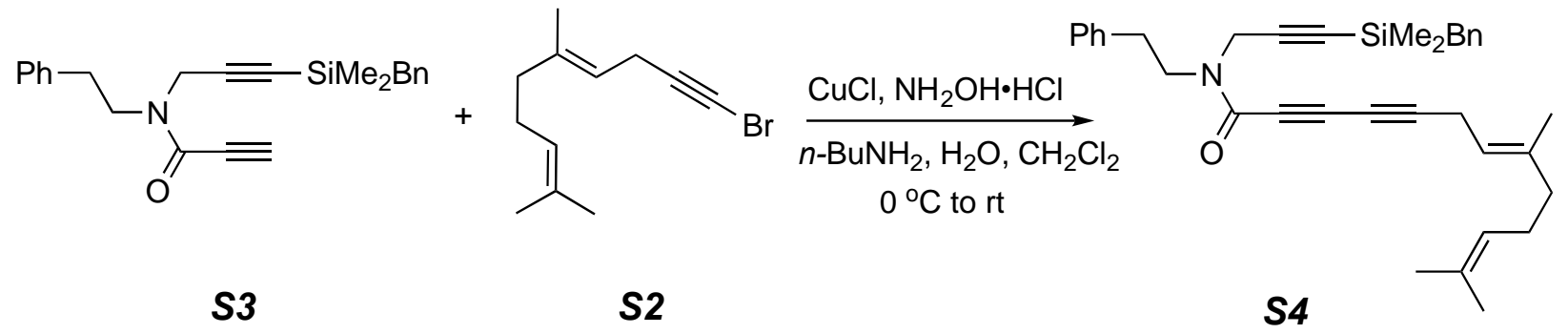

To a stirred solution of $40 \% n-\mathrm{BuNH}_{2} / \mathrm{H}_{2} \mathrm{O}(1 \mathrm{~mL})$ and $\mathrm{CH}_{2} \mathrm{Cl}_{2}(1 \mathrm{~mL})$ were added cuprous chloride (4.4 $\mathrm{mg}, 0.0445 \mathrm{mmol})$ and hydroxylamine hydrochloride $(12.4 \mathrm{mg}, 0.178 \mathrm{mmol})$ at $0{ }^{\circ} \mathrm{C}$. In the meantime, a solution of diyne $\mathbf{S 3}$ (32 $\mathrm{mg}, 0.089 \mathrm{mmol})$ in $\mathrm{CH}_{2} \mathrm{Cl}_{2}(1 \mathrm{~mL})$ was added in a dropwise fashion into the reaction vial. The reaction was stirred at $0{ }^{\circ} \mathrm{C}$ for $5 \mathrm{~min}$, and bromoalkyne $\mathbf{S 2}$ (42.9 $\left.\mathrm{mg}, 0.178 \mathrm{mmol}\right)$ in $\mathrm{CH}_{2} \mathrm{Cl}_{2}(1 \mathrm{~mL})$ was added slowly (1 drop per 2 seconds). After the addition, the reaction mixture was allowed to warm to ambient temperature for $30 \mathrm{~min}$ before it was quenched by the addition of $\mathrm{NH}_{4} \mathrm{Cl}(10$ $\mathrm{mL}$, satd aq). The layers were separated and the aqueous layer was extracted with $\mathrm{CH}_{2} \mathrm{Cl}_{2}(3 \times 15 \mathrm{~mL})$. The combined organic layers were washed with brine $(20 \mathrm{~mL})$, dried $\left(\mathrm{Na}_{2} \mathrm{SO}_{4}\right)$, and concentrated in vacuo. Flash column chromatography (silica gel, hexanes:EtOAc 5:1) afforded amide $\mathbf{S 4}$ as a colorless oil (28.0 mg, $61 \%)$.

Two rotamers for the tertiary amide (ca. 3:2) were evident in the NMR spectral data:

${ }^{1} \mathbf{H}$ NMR (500 MHz, $\left.\mathrm{CDCl}_{3}\right): \delta$ 7.34-7.27 (m, 2H, PhH), 7.26-7.16 (m, 5H, PhH), 7.09-7.02 (m, 3H, PhH), 5.20-5.14 (m, 1H, C7H), 5.11-5.05 (m, 1H, C11H), $4.22\left(\mathrm{~s}, 1.2 \mathrm{H}, \mathrm{C} \equiv \mathrm{CCH}_{2} \mathrm{~N}\right.$, major), $4.21(\mathrm{~s}, 0.8 \mathrm{H}$, $\mathrm{C} \equiv \mathrm{CCH}_{2} \mathrm{~N}$, minor), 3.83 (nfot, $J=7.6 \mathrm{~Hz}, 1.2 \mathrm{H}, \mathrm{NCH}_{2} \mathrm{CH}_{2}$, major), 3.67 (nfot, $J=7.8 \mathrm{~Hz}, 0.8 \mathrm{H}, \mathrm{NCH}_{2} \mathrm{CH}_{2}$, minor), 3.08 (br d, $J=6.8 \mathrm{~Hz}, 1.2 \mathrm{H}, \mathrm{C} \equiv \mathrm{CCH}_{2} \mathrm{C}=\mathrm{C}$, major), 3.06 (br d, $J=6.9 \mathrm{~Hz}, 0.8 \mathrm{H}, \mathrm{C} \equiv \mathrm{CCH} \mathrm{C}_{2} \mathrm{C}$, minor), 2.92 (nfot, $J=7.7 \mathrm{~Hz}, 1.2 \mathrm{H}, \mathrm{NCH}_{2} \mathrm{CH}_{2}$, major), 2.87 (nfot, $J=7.7 \mathrm{~Hz}, 0.8 \mathrm{H}, \mathrm{NCH}_{2} \mathrm{CH}_{2}$, minor),

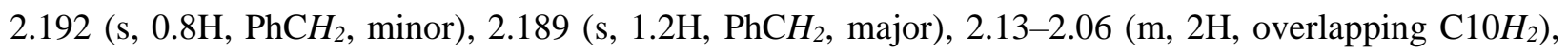
2.06-1.99 (m, 2H, overlapping $\left.\mathrm{C}^{2} H_{2}\right), 1.69$ (br s, 3H, overlapping $\left.\mathrm{C} 12 \mathrm{ECH} H_{3}\right), 1.66(\mathrm{dt}, J=1.3,0.7 \mathrm{~Hz}$, $1.8 \mathrm{H}, \mathrm{C} 8 \mathrm{CH}_{3}$, major), 1.64 (dt, $J=1.3,0.7 \mathrm{~Hz}, 1.2 \mathrm{H}, \mathrm{C} 8 \mathrm{CH}_{3}$, minor), 1.61 (br s, $3 \mathrm{H}$, overlapping

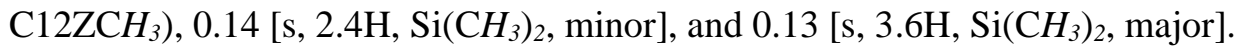

${ }^{13}$ C NMR (125 MHz, $\left.\mathrm{CDCl}_{3}\right): \delta 153.2,153.1,139.7,139.7,138.8,138.69,138.65,138.0,132.00,131.99$, $129.0,128.9,128.8,128.7,128.44,128.42,128.4,128.3,126.9,126.7,124.62,124.59,123.9,115.9,115.8$, $100.9,100.7,88.9,88.3,86.8,86.7,76.5,76.1,66.34,66.26,63.5,63.4,49.8,46.5,40.3,39.50,39.48,35.4$, $35.0,33.5,26.51,26.49,26.14,26.10,25.8$ (x2), 18.8, 18.7, 17.85 (x2), 16.41, 16.40, -2.08, and -2.11.

HRMS (ESI) $m / z:[\mathrm{M}+\mathrm{H}]^{+}$Calculated for $\mathrm{C}_{35} \mathrm{H}_{42} \mathrm{NOSi}^{+}$520.3030; Found 520.3010.

IR (neat): 2962, 2923, 2857, 2241, and $1637 \mathrm{~cm}^{-1}$. 
(E)-4-(Benzyldimethylsilyl)-5-(3,7-dimethylocta-2,6-dien-1-yl)-6-methoxy-2phenethylisoindolin-1-one (18a)
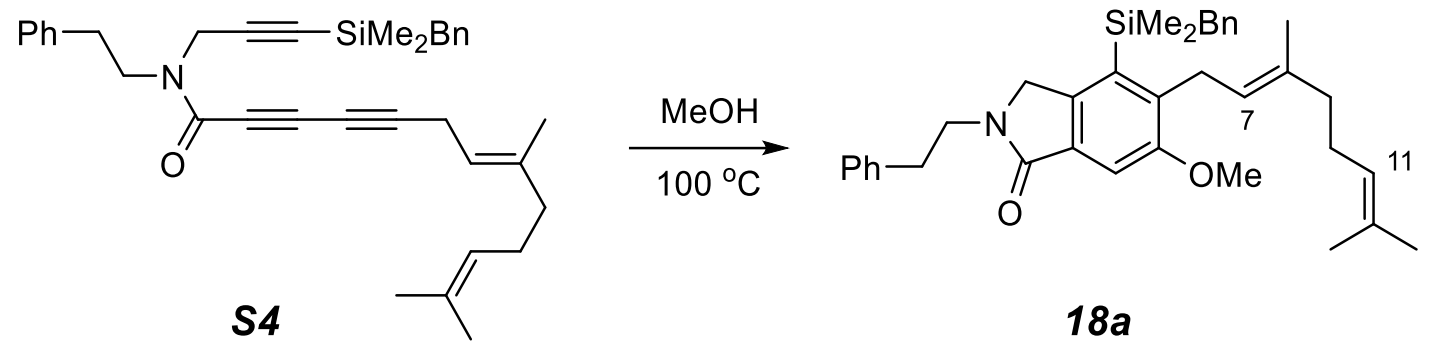

$18 a$

A solution of the amide $\mathbf{S 4}(100 \mathrm{mg}, 0.19 \mathrm{mmol})$ in methanol $(5 \mathrm{~mL})$ was heated in a screw-capped culture tube in an oil bath held at $100^{\circ} \mathrm{C}$. After $4 \mathrm{~h}$ the slightly yellow-colored solution was allowed to cool to room temperature and concentrated in vacuo. Flash column chromatography (silica gel, hexanes:EtOAc 4:1) afforded the lactam 18a (85 mg, 80\%) as a light yellow oil.

${ }^{1} \mathbf{H}$ NMR (500 MHz, $\left.\mathrm{CDCl}_{3}\right): \delta 7.35(\mathrm{~s}, 1 \mathrm{H}, \mathrm{Ar} H), 7.28$ (br t, $\left.J=8 \mathrm{~Hz}, 2 \mathrm{H}, \mathrm{Ph} H_{m}\right), 7.21(\mathrm{br} \mathrm{d}, J=$ $7 \mathrm{~Hz}, 2 \mathrm{H}, \mathrm{Ph} H_{o}$ ), 7.20 (tt, $J=6.5,1.4 \mathrm{~Hz}, 1 \mathrm{H}, \mathrm{Ph} H_{p}$ ), 7.12 (br dd, $J=7.4,7.4 \mathrm{~Hz}, 2 \mathrm{H}, \mathrm{Ph} H_{m}{ }^{\prime}$ ), 7.04 (tt, $J=6.7,1.3 \mathrm{~Hz}, 1 \mathrm{H}, \mathrm{Ph} H_{p}{ }^{\prime}$ ), 6.80 (br d, $J 7.4 \mathrm{~Hz}, 2 \mathrm{H}, \mathrm{Ph} H_{o}{ }^{\prime}$ ), 5.05 (tqq, $J=6.6,1.3,1.3$ $\mathrm{Hz}, 1 \mathrm{H}, \mathrm{C} 6 H$ ) , 4.96 (ttq, $J=5.9,1.3,1.3 \mathrm{~Hz}, 1 \mathrm{H}, \mathrm{C} 2 H$ ), 3.87 (s, 3H, OCH3), 3.78 (br t, $J=7.5 \mathrm{~Hz}$, $\left.2 \mathrm{H}, \mathrm{NCH}_{2} \mathrm{CH}_{2}\right), 3.75\left(\mathrm{~s}, 2 \mathrm{H}, \mathrm{ArCH}_{2} \mathrm{~N}\right), 3.52\left(\mathrm{~d}, J=5.9 \mathrm{~Hz}, 2 \mathrm{H}, \mathrm{ArCH}_{2} \mathrm{CH}=\mathrm{C}\right), 2.89$ (t, $J=7.4 \mathrm{~Hz}$, 2H, $\mathrm{NCH}_{2} \mathrm{CH}_{2}$ ), 2.30 (s, 2H, $\mathrm{PhCH}_{2}$ ), 2.04 (br dt, $J=7,7 \mathrm{~Hz}, 2 \mathrm{H}, \mathrm{C} 10 H_{2}$ ), 1.99 (br t, $J=7.5 \mathrm{~Hz}$, $\left.2 \mathrm{H}, \mathrm{C}_{2} \mathrm{H}_{2}\right), 1.74\left(\mathrm{dt}, J=1.2 \mathrm{~Hz}, 3 \mathrm{H}, \mathrm{C} 12 \mathrm{ECH}_{3}\right), 1.61\left(\mathrm{dt}, J=1.1,1.1 \mathrm{~Hz}, 3 \mathrm{H}, \mathrm{C}_{\mathrm{CH}}\right.$ ), 1.55 (br s,

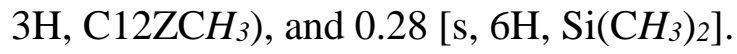

${ }^{13} \mathrm{C}$ NMR (125 MHz, $\left.\mathrm{CDCl}_{3}\right): \delta$ 168.5, 158.2, 149.2, 141.2, 139.5, 139.3, 139.2, 135.5, 132.2, $131.4,128.9,128.7,128.4,128.3,126.6,124.5,124.3,123.7,106.0,55.8,52.8,44.0,39.7,35.2$, $30.4,27.1,26.7,25.8,17.8,16.7$, and 0.8 .

HRMS (ESI) $m / z:[\mathrm{M}+\mathrm{H}]^{+}$Calculated for $\mathrm{C}_{36} \mathrm{H}_{46} \mathrm{NO}_{2} \mathrm{Si}^{+}$552.3292; Found 552.3269.

IR (neat): 3057, 2984, 2937, 2856, 1734, and $1687 \mathrm{~cm}^{-1}$. 
Attempted oxidation of 18 a to give, instead, the protio-desilylated product (E)-5-(3,7-Dimethylocta-2,6-dien-1-yl)-6-methoxy-2-phenethylisoindolin-1-one (19)

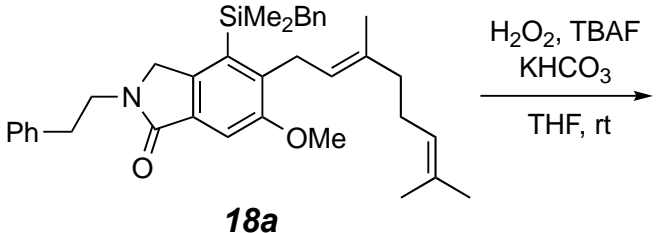

$18 a$

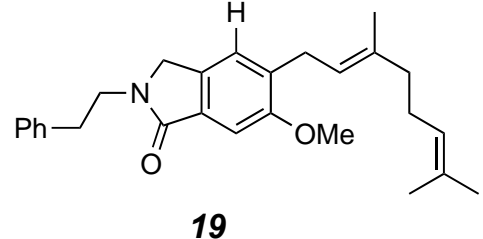

19

To a stirred solution of $\mathbf{1 8 a}(55 \mathrm{mg}, 0.1 \mathrm{mmol})$ in THF $(3 \mathrm{~mL})$ were added $\quad(20 \quad \mathrm{mg}, \quad 0.2$ mmol), $\mathrm{H}_{2} \mathrm{O}_{2}$ (30 wt.\% in $\mathrm{H}_{2} \mathrm{O}, 0.24 \mathrm{~mL}, 2 \mathrm{mmol}$ ), and TBAF (1.0 M in THF, $0.4 \mathrm{~mL}, 0.4 \mathrm{mmol}$ ). The resulting mixture was stirred at room temperature for $3 \mathrm{~h}$ before it was quenched by the addition of $\mathrm{NaHCO}_{3}(10 \mathrm{~mL}$, satd aq). The layers were separated and the aqueous layer was extracted with $\mathrm{CH}_{2} \mathrm{Cl}_{2}(3 \times 10 \mathrm{~mL})$. The combined organic layers were washed with brine $(20 \mathrm{~mL})$, dried $\left(\mathrm{Na}_{2} \mathrm{SO}_{4}\right)$, and concentrated in vacuo. Flash column chromatography (silica gel, hexanes:EtOAc 4:1) afforded $19(28 \mathrm{mg}, 65 \%)$ as a colorless oil.

${ }^{1} \mathbf{H}$ NMR (400 MHz, $\left.\mathrm{CDCl}_{3}\right): \delta 7.28\left(\mathrm{~m}, 2 \mathrm{H}, \mathrm{Ph} H_{m}\right), 7.26\left(\mathrm{~s}, 1 \mathrm{H}, \mathrm{C} 4{ }^{\prime} H\right), 7.23\left(\mathrm{~m}, 2 \mathrm{H}, \mathrm{Ph} H_{o}\right), 7.19$ (tt, $\left.J=7.1,1.4 \mathrm{~Hz}, 1 \mathrm{H}, \mathrm{Ph} H_{p}\right), 7.12$ (s, 1H, C4H), 5.29 (dqt, $J=7.1,1.1,1.1 \mathrm{~Hz}, 1 \mathrm{H}, \mathrm{C} 7 H$ ), 5.10 (dqq, $J=6.8,1.4,1.4 \mathrm{~Hz}, 1 \mathrm{H}, \mathrm{C} 7 \mathrm{H}), 4.10\left(\mathrm{~s}, 2 \mathrm{H}, \mathrm{ArCH}_{2} \mathrm{~N}\right.$ ), 3.89 (s, 3H, OCH $), 3.86$ (nfot, 2H, $\mathrm{NCH}_{a} H_{a} \cdot \mathrm{CH}_{\mathrm{b}} \mathrm{H}_{\mathrm{b}} \mathrm{Ph}$ ), 3.37 (dqt, 2H, $J=7.2,1.0,1.0 \mathrm{~Hz}, 2 \mathrm{H}, \mathrm{ArCH}_{2} \mathrm{CH}=\mathrm{C}$ ), 2.97 (nfot, 2H, $\mathrm{NCH}_{\mathrm{a}} \mathrm{H}_{\mathrm{a}}{ }^{\mathrm{C}} \mathrm{CH}_{b} \mathrm{H}_{b} \cdot \mathrm{Ph}$ ), 2.09 (br dt, $J=\sim 7, \sim 7 \mathrm{~Hz}, 2 \mathrm{H}, \mathrm{C} 10 H_{2}$ ), 2.06 (br t, $J=\sim 7 \mathrm{~Hz}, 2 \mathrm{H}, \mathrm{C} H_{2}$ ), $1.68\left(\mathrm{dt}, J=1.1,1.1 \mathrm{~Hz}, 3 \mathrm{H}, \mathrm{C} 12 \mathrm{ECH}_{3}\right), 1.66(\mathrm{dt}, J=1.4,1.4 \mathrm{~Hz}, 3 \mathrm{H}, \mathrm{C} 8 \mathrm{CH} 3$ ), and 1.59 (br d, $J$ $\left.=1.4 \mathrm{~Hz}, 3 \mathrm{H}, \mathrm{C} 12 \mathrm{ZCH}_{3}\right)$.

${ }^{13}$ C NMR (125 MHz, $\left.\mathrm{CDCl}_{3}\right): \delta 169.0,157.8,139.1,137.1,134.7,133.4,131.8,131.6,128.9$, $128.7,126.6,124.4,123.0,121.7,104.4,55.86,50.4$, 44.3, 39.9, 35.2, 28.8, 26.7, 25.9, 17.9, and 16.2 .

HRMS (ESI) $m / z:[\mathrm{M}+\mathrm{H}]^{+}$Calculated for $\mathrm{C}_{27} \mathrm{H}_{34} \mathrm{NO}_{2}{ }^{+} 404.2584$; Found 404.2564.

IR (neat): 2960, 2929, 2856, 1685, and $1598 \mathrm{~cm}^{-1}$. 


\section{1-(3-Bromoprop-1-yn-1-yl)-1,1,2,2,2-pentamethyldisilane (S5)}
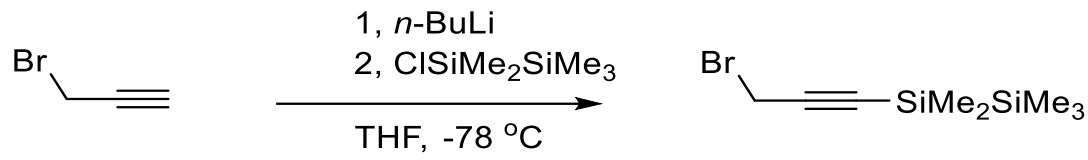

S5

To a stirred solution of propargyl bromide ( $80 \mathrm{wt} \%$ in toluene, $781 \mathrm{mg}, 0.6 \mathrm{~mL}, 5.25 \mathrm{mmol})$ in THF $(5 \mathrm{~mL})$ was added a solution of $n-\operatorname{BuLi}(2.5 \mathrm{M}$ in hexanes, $2.0 \mathrm{~mL}, 5.0 \mathrm{mmol})$ dropwise at $78^{\circ} \mathrm{C}$. The reaction mixture was stirred at $-78^{\circ} \mathrm{C}$ for $30 \mathrm{~min}$, followed by the addition of 1-chloro1,1,2,2,2-pentamethyldisilane ( $870 \mathrm{mg}, 5.25 \mathrm{mmol})$ in THF $(2 \mathrm{~mL})$. The reaction mixture was kept at $-78{ }^{\circ} \mathrm{C}$ for an additional $30 \mathrm{~min}$, allowed to warm to $0{ }^{\circ} \mathrm{C}$, and stirred for an additional $1 \mathrm{~h}$. The light yellow reaction mixture was quenched by the addition of saturated $\mathrm{NH}_{4} \mathrm{Cl}$ solution $(5 \mathrm{~mL})$, and the aqueous layer was extracted with hexanes $(3 \times 10 \mathrm{~mL})$. The combined organic layer was washed with brine $(10 \mathrm{~mL})$, dried with $\mathrm{MgSO}_{4}$, filtered, and concentrated. The resulting residue was purified by flash column chromatography (100\% hexanes) to give silane $\mathbf{S 5}$ as a colorless oil $(1.0 \mathrm{~g}, 82 \%)$.

${ }^{1} \mathrm{H}$ NMR (400 MHz, $\left.\mathrm{CDCl}_{3}\right): \delta 3.94\left(\mathrm{~s}, 2 \mathrm{H}, \mathrm{BrCH}_{2}\right), 0.20$ [s, 6H, $\left.\mathrm{Si}\left(\mathrm{CH}_{3}\right)_{2} \mathrm{Si}\left(\mathrm{CH}_{3}\right)_{3}\right]$, and 0.13 [s, 9H, $\left.\mathrm{Si}\left(\mathrm{CH}_{3}\right)_{2} \mathrm{Si}\left(\mathrm{CH}_{3}\right)_{3}\right]$.

${ }^{13}$ C NMR (101 MHz, $\left.\mathrm{CDCl}_{3}\right): \delta 102.2,91.9,15.2,-2.5$, and -3.2.

HRMS (CI) $m / z:\left[\mathrm{M}+\mathrm{NH}_{4}\right]^{+}$Calcd for $\mathrm{C}_{8} \mathrm{H}_{21}{ }^{81} \mathrm{BrNSi}^{+}$268.0370; Found 268.0375.

IR (neat): 2986, 2957 and $2894 \mathrm{~cm}^{-1}$. 
$N$-(3-(1,1,2,2,2-Pentamethyldisilaneyl)prop-2-yn-1-yl)- $N$-phenethylpropiolamide (S6)

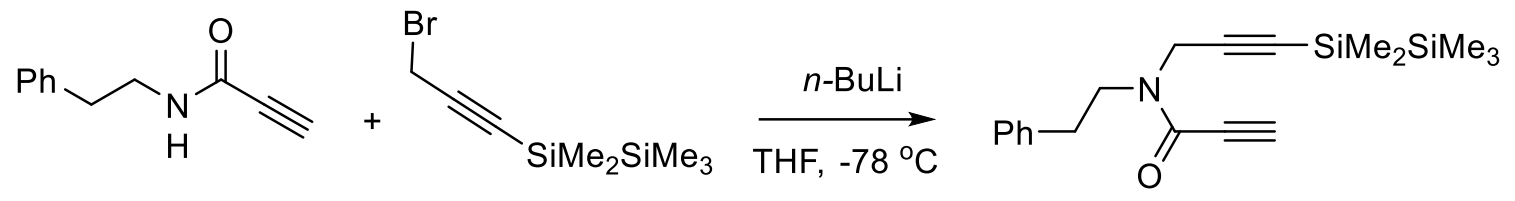

S5

S6

To a stirred solution of $N$-phenethylpropiolamide $(160 \mathrm{mg}, 0.92 \mathrm{mmol})$ in THF (4 mL) was added a solution of $n$-BuLi $(2.5 \mathrm{M}$ in hexanes, $0.36 \mathrm{~mL}, 0.9 \mathrm{mmol})$ dropwise at $-78{ }^{\circ} \mathrm{C}$. The resulting solution was stirred for an additional $30 \mathrm{~min}$, followed by the dropwise addition of bromide $\mathbf{S 5}$ (228 mg, $0.92 \mathrm{mmol}$ ) at $-78^{\circ} \mathrm{C}$. The reaction mixture was allowed to warm to ambient temperature and further stirred for $1 \mathrm{~h}$. The light yellow slurry was quenched with satd $\mathrm{NH}_{4} \mathrm{Cl}$ solution, extracted with hexanes $(3 \times 10 \mathrm{~mL})$, and washed with brine $(20 \mathrm{~mL})$. The combined organic layer was dried and concentrated. The residue was purified by flash chromatography (hexanes:EtOAc 2:1) to give the amide $\mathbf{S 6}$ as a light yellow oil (250 $\mathrm{mg}, 80 \%)$.

Two rotamers for the tertiary amide (ca. 3:2) were evident in the NMR spectral data:

${ }^{1} \mathbf{H}$ NMR $\left(500 \mathrm{MHz} \mathrm{CDCl}_{3}\right.$ ): $\delta 7.31$ (br dd, $J=7.3,7.3 \mathrm{~Hz}, 0.8 \mathrm{H}, \mathrm{Ph} H_{m}$, minor), 7.30 (br dd, $J=$ 7, $7 \mathrm{~Hz}, 1.2 \mathrm{H}, \mathrm{Ph} H_{m}$, major), 7.27-7.16 (m, 3H, $\mathrm{Ph} H_{o}$ and $\left.\mathrm{Ph} H_{p}\right), 4.28\left(\mathrm{~s}, 2 \mathrm{H}, \mathrm{C} \equiv \mathrm{CCH} \mathrm{C}_{2} \mathrm{~N}\right), 3.93$ (nfot, $J=7.5 \mathrm{~Hz}, 1.2 \mathrm{H}, \mathrm{NCH}_{2} \mathrm{CH}_{2}$, major), 3.72 (nfot, $J=7.5 \mathrm{~Hz}, 0.8 \mathrm{H}, \mathrm{NCH}_{2} \mathrm{CH}_{2}$, minor), 3.12 (s, 0.4H, $\mathrm{C} \equiv \mathrm{CH}$, minor), 3.08 (s, 0.6H, $\mathrm{C} \equiv \mathrm{CH}$, major), 2.98 (nfot, $J=8.0 \mathrm{~Hz}, 1.2 \mathrm{H}, \mathrm{NCH}_{2} \mathrm{CH}_{2}$, major), 2.92 (nfot, $J=8.0 \mathrm{~Hz}, 0.8 \mathrm{H}, \mathrm{NCH}_{2} \mathrm{CH}_{2}$, minor), 0.20 [s, 3.6H, $\mathrm{Si}\left(\mathrm{CH}_{3}\right)_{2}$, major], 0.19 [s, 2.4H, $\mathrm{Si}\left(\mathrm{CH}_{3}\right)_{2}$, minor], and $0.11\left[\mathrm{~s}, 9 \mathrm{H}, \mathrm{Si}\left(\mathrm{CH}_{3}\right)_{3}\right]$.

${ }^{13} \mathrm{C}$ NMR $\left(125 \mathrm{MHz}, \mathrm{CDCl}_{3}\right): \delta 153.0,152.9,138.7,138.0,128.92,128.90,128.9,128.7,126.9$, $126.7,101.2,101.2,89.5,89.1,79.3,78.8,75.7,75.6,49.7,46.5,40.5,35.3,35.0,33.6,-2.45$, $2.48,-3.0$, and -3.1 .

HRMS (ESI) $m / z:[\mathrm{M}+\mathrm{H}]^{+}$Calculated for $\mathrm{C}_{19} \mathrm{H}_{28} \mathrm{NOSi}_{2}{ }^{+} 342.1704$, found 342.1688.

IR (neat): 3054, 2986, 2953, 2235, and $1637 \mathrm{~cm}^{-1}$. 


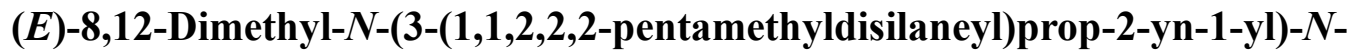 phenethyltrideca-7,11-dien-2,4-diynamide (S7)}

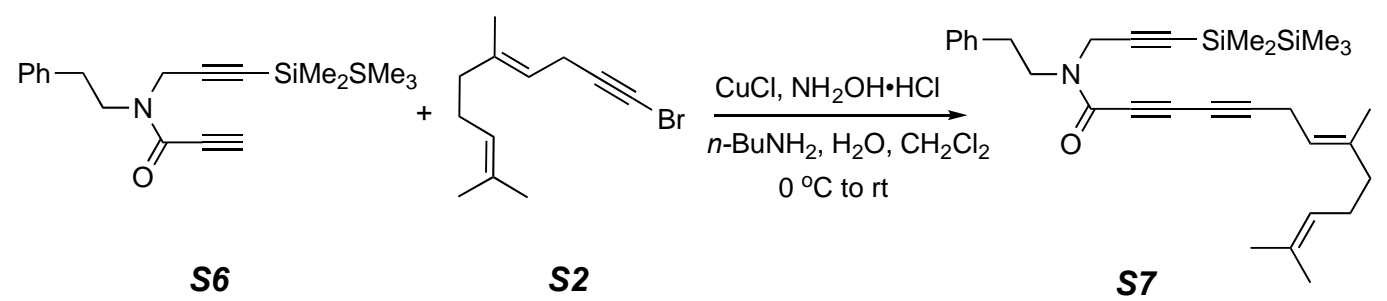

To a stirred solution of $40 \% n-\mathrm{BuNH}_{2} / \mathrm{H}_{2} \mathrm{O}(1 \mathrm{~mL})$ and $\mathrm{CH}_{2} \mathrm{Cl}_{2}(1 \mathrm{~mL})$ were added cuprous chloride (4.0 $\mathrm{mg}, 0.04 \mathrm{mmol})$ and hydroxylamine hydrochloride $(12 \mathrm{mg}, 0.2 \mathrm{mmol})$ at $0{ }^{\circ} \mathrm{C}$. In the meantime, a solution of diyne $\mathbf{S 6}(70 \mathrm{mg}, 0.2 \mathrm{mmol})$ in $\mathrm{CH}_{2} \mathrm{Cl}_{2}(1 \mathrm{~mL})$ was added in a dropwise fashion into the reaction vial. The reaction was stirred at $0{ }^{\circ} \mathrm{C}$ for $5 \mathrm{~min}$, and bromoalkyne $\mathbf{S 2}(72 \mathrm{mg}, 0.3 \mathrm{mmol})$ in $\mathrm{CH}_{2} \mathrm{Cl}_{2}(1 \mathrm{~mL})$ was added slowly ( 1 drop per 2 seconds). After the addition, the reaction mixture was allowed to warm to ambient temperature for $30 \mathrm{~min}$ before it was quenched by the addition of $\mathrm{NH}_{4} \mathrm{Cl}(10 \mathrm{~mL}$, satd aq). The layers were separated and the aqueous layer was extracted with $\mathrm{CH}_{2} \mathrm{Cl}_{2}(3 \times 10 \mathrm{~mL})$. The combined organic layers were washed with brine $(20 \mathrm{~mL})$, dried $\left(\mathrm{Na}_{2} \mathrm{SO}_{4}\right)$, and concentrated in vacuo. Flash column chromatography (silica gel, hexanes:EtOAc 5:1) afforded amide $\mathbf{S 7}$ as a colorless oil (80 mg, 80\%).

Two rotamers for the tertiary amide (ca. 3:2) were evident in the NMR spectral data:

${ }^{1}$ H NMR $\left(500 \mathrm{MHz}, \mathrm{CDCl}_{3}\right.$ ): $\delta 7.31$ (br dd, $J=7.3,7.3 \mathrm{~Hz}, 0.8 \mathrm{H}, \mathrm{Ph} H_{m}$, minor), 7.30 (br dd, $J=7,7 \mathrm{~Hz}$, 1.2H, $\mathrm{Ph} H_{m}$, major), 7.25-7.20 (m, 3H, $\mathrm{Ph} H_{o}$ and $\mathrm{Ph} H_{p}$ ), 5.17 (br t, $J=7.0 \mathrm{~Hz}, 0.6 \mathrm{H}, \mathrm{C} 7 \mathrm{H}$, maj), 5.16 (br $\mathrm{t}, J=7.0 \mathrm{~Hz}, 0.4 \mathrm{H}, \mathrm{C} 7 \mathrm{H}, \mathrm{min}), 5.11-5.05(\mathrm{~m}, 1 \mathrm{H}, \mathrm{C} 11 \mathrm{H}), 4.26\left(\mathrm{~s}, 1.2 \mathrm{H}, \mathrm{C} \equiv \mathrm{CCH}_{2} \mathrm{~N}\right.$, major), $4.24(\mathrm{~s}, 0.8 \mathrm{H}$, $\mathrm{C} \equiv \mathrm{CCH}_{2} \mathrm{~N}$, minor), 3.89 (nfot, $J=7.4 \mathrm{~Hz}, 1.2 \mathrm{H}, \mathrm{NCH}_{2} \mathrm{CH}_{2}$, major), 3.71 (nfot, $J=7.7 \mathrm{~Hz}, 0.8 \mathrm{H}, \mathrm{NCH}_{2} \mathrm{CH}_{2}$, minor), 3.07 (br d, $J=7.0 \mathrm{~Hz}, 1.2 \mathrm{H}, \mathrm{C} \equiv \mathrm{CCH}_{2} \mathrm{C}=\mathrm{C}$, major), 3.05 (br d, $J=7.0 \mathrm{~Hz}, 0.8 \mathrm{H}, \mathrm{C} \equiv \mathrm{CCH} \mathrm{C}_{2} \mathrm{C}$, minor), 2.96 (nfot, $J=8.1 \mathrm{~Hz}, 1.2 \mathrm{H}, \mathrm{NCH}_{2} \mathrm{CH}_{2}$, major), 2.90 (nfot, $J=8.1 \mathrm{~Hz}, 0.8 \mathrm{H}, \mathrm{NCH}_{2} \mathrm{CH}_{2}$, minor),

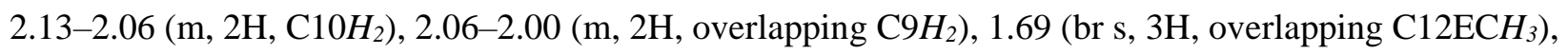
$1.65\left(\mathrm{dt}, J=1.2,0.8 \mathrm{~Hz}, 1.8 \mathrm{H}, \mathrm{C} 8 \mathrm{CH}_{3}\right.$, major), 1.64 (dt, $J=1.2,0.8 \mathrm{~Hz}, 1.2 \mathrm{H}, \mathrm{C} 8 \mathrm{CH} H_{3}$, minor), 1.61 (br s, $3 \mathrm{H}$, overlapping $\left.\mathrm{C}^{2} 2 \mathrm{ZCH}_{3}\right), 0.20$ [s, 3.6H, $\mathrm{Si}\left(\mathrm{CH}_{3}\right)_{2}$, minor], 0.19 [s, 2.4H, $\mathrm{Si}\left(\mathrm{CH}_{3}\right)_{2}$, major], 0.11 [s, 3.6H, $\mathrm{Si}\left(\mathrm{CH}_{3}\right)_{3}$, minor], and 0.10 [s, $5.4 \mathrm{H}, \mathrm{Si}\left(\mathrm{CH}_{3}\right)_{3}$, major].

${ }^{13} \mathrm{C}$ NMR (125 MHz, $\left.\mathrm{CDCl}_{3}\right): \delta 153.18,153.11,139.7,138.7,138.0,132.01,132.00,129.0,128.9,128.8$, 128.7, 126.9, 126.6, 123.9, 116.0, 115.9, 101.3, 89.6, 89.0, 86.63, 86.61, 76.4, 76.0, 66.4, 66.3, 63.5, 63.4, $49.7,46.5,40.4,39.51,39.49,35.5,35.1,33.6,26.52$, 26.50, 25.9, 18.8, 18.7, 17.9, 16.42, 16.40, -2.4, -2.5, -3.0 , and -3.1 .

HRMS (ESI) $m / z:[\mathrm{M}+\mathrm{H}]^{+}$Calculated for $\mathrm{C}_{31} \mathrm{H}_{44} \mathrm{NOSi}_{2}{ }^{+}$502.2956; Found 502.2935.

IR (neat): 2955, 2929, 2895, 2856, 2242, and $1638 \mathrm{~cm}^{-1}$. 
(E)-5-(3,7-Dimethylocta-2,6-dien-1-yl)-6-methoxy-4-(1,1,2,2,2-pentamethyldisilaneyl)-2phenethylisoindolin-1-one (18b)

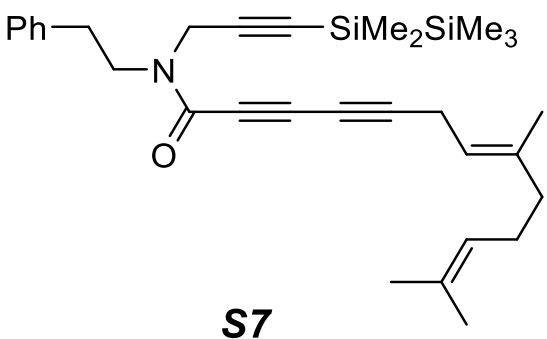

S7

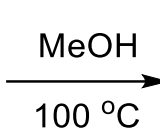

A solution of the amide $\mathbf{S 7}(100 \mathrm{mg}, 0.2 \mathrm{mmol})$ in methanol $(4 \mathrm{~mL})$ was heated in a screw-capped culture tube in an oil bath held at $100^{\circ} \mathrm{C}$. After $4 \mathrm{~h}$ the slightly yellow-colored solution was allowed to cool to room temperature and concentrated in vacuo. Flash column chromatography (silica gel, hexanes:EtOAc 4:1) afforded the lactam $\mathbf{1 8 b}(80 \mathrm{mg}, 75 \%)$ as a light yellow oil.

${ }^{1}$ H NMR (500 MHz, $\mathrm{CDCl}_{3}$ ): $\delta 7.294$ (s, 1H, ArH), 7.287 (br t, $J=7.3 \mathrm{~Hz}, 2 \mathrm{H}, \mathrm{PhHm}$ ), 7.24 (br d, $\left.J=7.3 \mathrm{~Hz}, 2 \mathrm{H}, \mathrm{Ph} H_{o}\right), 7.21\left(\mathrm{tt}, J=7.2,1.4 \mathrm{~Hz}, \mathrm{Ph} H_{p}\right), 5.06$ (tqq, $J=6.8,1.2,1.2 \mathrm{~Hz}, 1 \mathrm{H}, \mathrm{C} 6 H$ ), 4.90 (ttq, $J=5.7,1.2,1.2 \mathrm{~Hz}, 1 \mathrm{H}, \mathrm{C} 2 \mathrm{H}), 4.05\left(\mathrm{~s}, 2 \mathrm{H}, \mathrm{ArCH}_{2} \mathrm{~N}\right), 3.87$ (t, $J=7.2 \mathrm{~Hz}, 2 \mathrm{H}, \mathrm{NCH}_{2} \mathrm{CH}_{2}$ ), $3.84\left(\mathrm{~s}, 3 \mathrm{H}, \mathrm{OCH}_{3}\right), 3.38$ (d, $\left.J=5.5 \mathrm{~Hz}, 2 \mathrm{H}, \mathrm{ArCH}_{2} \mathrm{CH}=\mathrm{C}\right), 2.99$ (t, $J=7.2 \mathrm{~Hz}, 2 \mathrm{H}, \mathrm{NCH}_{2} \mathrm{CH}_{2}$ ), 2.05 (br dt, $J=7.2,7.2 \mathrm{~Hz}, 2 \mathrm{H}, \mathrm{C} 5 \mathrm{H}_{2}$ ), 1.99 (br t, $J=7.4 \mathrm{~Hz}, 2 \mathrm{H}, \mathrm{C} 4 \mathrm{H}_{2}$ ), 1.72 (dt, $J=1.1,1.1 \mathrm{~Hz}$, $3 \mathrm{H}, \mathrm{C} \mathrm{ECH}$ ), 1.64 (dt, $J=1.1,1.1 \mathrm{~Hz}, 3 \mathrm{H}, \mathrm{C} 3 \mathrm{CH}_{3}$ ), 1.57 (br s, 3H, C7ZCH3), 0.35 [s, 6H, $\left.\mathrm{Si}\left(\mathrm{CH}_{3}\right)_{2}\right]$, and 0.28 [s, 9H, $\left.\mathrm{Si}\left(\mathrm{CH}_{3}\right)_{3}\right]$.

${ }^{13} \mathrm{C}$ NMR $\left(125 \mathrm{MHz}, \mathrm{CDCl}_{3}\right): \delta 168.8,158.1,141.0,139.3,139.0,135.2,133.4,131.6,131.4$, 128.9, 128.8, 126.7, 124.4, 124.0, 105.4, 55.8, 53.1, 44.2, 39.7, 35.3, 31.8, 26.7, 25.9, 17.8, 16.6, 0.1 , and -1.4.

HRMS (ESI) $m / z:[\mathrm{M}+\mathrm{H}]^{+}$Calculated for $\mathrm{C}_{32} \mathrm{H}_{48} \mathrm{NO}_{2} \mathrm{Si}_{2}+534.3218$; Found 534.3195.

IR (neat): 2984, 2940, 2896, and $1733 \mathrm{~cm}^{-1}$. 
Attempted oxidation of $18 \mathrm{~b}$ to give, instead, the proto-desilylated (E)-5-(3,7-Dimethylocta2,6-dien-1-yl)-6-methoxy-2-phenethylisoindolin-1-one (19)

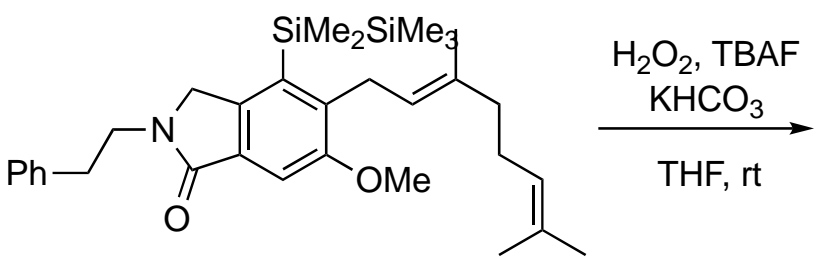

$18 b$<smiles>COc1cc2c(cc1C/C=C(\C)CCC=C(C)C)CN(CCc1ccccc1)C2=O</smiles>

19

To a stirred solution of $\mathbf{1 8 b}$ (32 $\mathrm{mg}, 0.06 \mathrm{mmol})$ in THF (3 mL) were added $\mathrm{KHCO}_{3}(12 \mathrm{mg}, 0.12$ mmol), $\mathrm{H}_{2} \mathrm{O}_{2}\left(30\right.$ wt. $\%$ in $\mathrm{H}_{2} \mathrm{O}, 72 \mu \mathrm{L}, 0.6 \mathrm{mmol}$ ), and TBAF (1.0 M in THF, $\left.240 \mu \mathrm{L}, 0.24 \mathrm{mmol}\right)$ at room temperature. The resulting mixture was stirred at room temperature for $2 \mathrm{~h}$ before it was quenched by the addition of $\mathrm{NaHCO}_{3}(10 \mathrm{~mL}$, satd aq). The layers were separated and the aqueous layer was extracted with $\mathrm{CH}_{2} \mathrm{Cl}_{2}(3 \times 10 \mathrm{~mL})$. The combined organic layers were washed with brine $(20 \mathrm{~mL})$, dried $\left(\mathrm{Na}_{2} \mathrm{SO}_{4}\right)$, and concentrated in vacuo. Flash column chromatography (silica gel, hexanes:EtOAc 4:1) afforded $19(20 \mathrm{mg}, 80 \%)$ as a colorless oil. 
IIc. Experimental details for preparation and characterization data for S8-S13 and 13-3

\section{3-(Dimethylsilyl)- $N$-phenethylprop-2-yn-1-amine (13)}

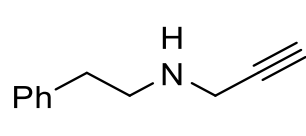

12

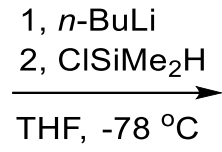

THF, $-78^{\circ} \mathrm{C}$

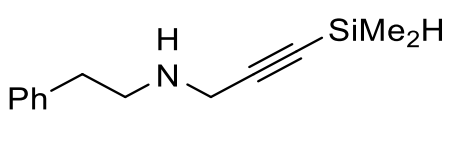

13

To a stirred solution of $12(800 \mathrm{mg}, 5.0 \mathrm{mmol})$ in THF $(10 \mathrm{~mL})$ was added a solution of $n$-BuLi $(2.5 \mathrm{M}$ in hexanes, $2.0 \mathrm{~mL}, 5.0 \mathrm{mmol})$ dropwise at $-78^{\circ} \mathrm{C}$. The reaction mixture was stirred at $78{ }^{\circ} \mathrm{C}$ for $30 \mathrm{~min}$, followed by the addition of chlorodimethylsilane $(0.67 \mathrm{~mL}, 6 \mathrm{mmol})$ in THF (2 $\mathrm{mL}$ ). The resulting mixture was allowed to warm to $0^{\circ} \mathrm{C}$ and stirred for an additional 30 min before it was quenched by the addition of $\mathrm{NH}_{4} \mathrm{Cl}(15 \mathrm{~mL}$, satd aq). The layers were separated and the aqueous layer was extracted with $\mathrm{CH}_{2} \mathrm{Cl}_{2}(3 \times 20 \mathrm{~mL})$. The combined organic layers were washed with brine $(20 \mathrm{~mL})$, dried $\left(\mathrm{Na}_{2} \mathrm{SO}_{4}\right)$, and concentrated in vacuo. Flash column chromatography (silica gel, hexanes:EtOAc 4:1) afforded 13 (960 mg, 88\%) as a light yellow oil.

${ }^{1} \mathbf{H}$ NMR (400 MHz, $\left.\mathrm{CDCl}_{3}\right): \delta 7.33-7.27\left(\mathrm{~m}, 2 \mathrm{H}, \mathrm{Ph} H_{m}\right), 7.23-7.19\left(\mathrm{~m}, 3 \mathrm{H}, \mathrm{Ph} H_{o}\right.$ and $\left.\mathrm{Ph} H_{p}\right)$, 4.12 [sept, $J=3.8 \mathrm{~Hz}, 1 \mathrm{H}, \mathrm{SiH}\left(\mathrm{CH}_{3}\right)_{2}$ ] $3.45\left(\mathrm{~s}, 1 \mathrm{H}, \mathrm{C} \equiv \mathrm{CCH}_{2} \mathrm{~N}\right), 2.97$ (br t, $J=7.1 \mathrm{~Hz}, 2 \mathrm{H}$, $\mathrm{NCH}_{2} \mathrm{CH}_{2}$ ), 2.82 (t, $J=7.0 \mathrm{~Hz}, 2 \mathrm{H}, \mathrm{NCH}_{2} \mathrm{CH}_{2}$ ), 1.39 (br s, $1 \mathrm{H}, \mathrm{NH}$ ), and 0.22 [d, $J=3.8 \mathrm{~Hz}, 6 \mathrm{H}$, $\left.\mathrm{SiH}\left(\mathrm{CH}_{3}\right)_{2}\right]$.

${ }^{13}$ C NMR (101 MHz, $\left.\mathrm{CDCl}_{3}\right): \delta$ 139.9, 128.8, 128.6, 126.4, 106.0, 85.1, 49.9, 39.3, 36.2, and -2.8.

HRMS (ESI) $m / z:[\mathrm{M}+\mathrm{H}]^{+}$Calcd for $\mathrm{C}_{13} \mathrm{H}_{20} \mathrm{NSi}^{+}$218.1360; Found 218.1351.

IR (neat): 3062, 3034, 3028, 2928, 2962, 2874, 2171 (s), $2136(\mathrm{~m}), 1652$, and $1600 \mathrm{~cm}^{-1}$. 
(E)-8,12-Dimethyltrideca-7,11-dien-2,4-diyn-1-ol (16a)

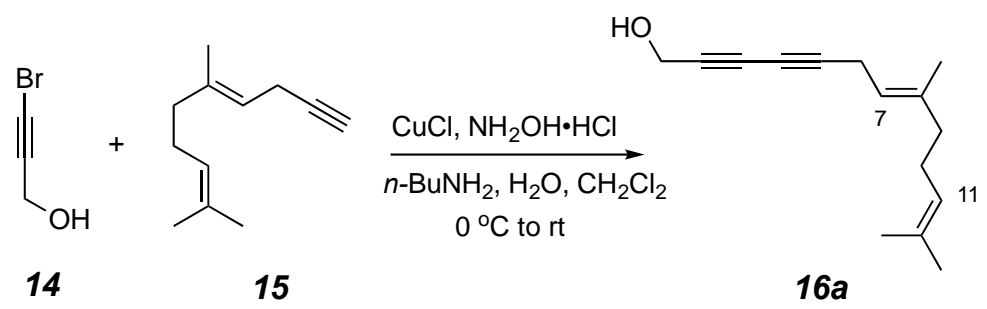

To a stirred solution of $40 \% n-\mathrm{BuNH}_{2} / \mathrm{H}_{2} \mathrm{O}(5 \mathrm{~mL})$ and $\mathrm{CH}_{2} \mathrm{Cl}_{2}(5 \mathrm{~mL})$ were added cuprous chloride $(61 \mathrm{mg}, 0.61 \mathrm{mmol})$ and hydroxylamine hydrochloride $(214.5 \mathrm{mg}, 3.08 \mathrm{mmol})$ at $0{ }^{\circ} \mathrm{C}$. In the meantime, a solution of dienyne $15(500 \mathrm{mg}, 3.08 \mathrm{mmol})$ in $\mathrm{CH}_{2} \mathrm{Cl}_{2}(2 \mathrm{~mL})$ was added in a dropwise fashion into the reaction vial. The reaction was stirred at $0{ }^{\circ} \mathrm{C}$ for $5 \mathrm{~min}$, and a solution of 3-bromoprop-2-yn-1-ol 14 (492.6 mg, $3.7 \mathrm{mmol})$ in $\mathrm{CH}_{2} \mathrm{Cl}_{2}(2 \mathrm{~mL})$ was added slowly (1 drop per 2 seconds). After the addition, the reaction mixture was allowed to warm to ambient temperature for $30 \mathrm{~min}$ before it was quenched by the addition of $\mathrm{NH}_{4} \mathrm{Cl}(10 \mathrm{~mL}$, satd aq). The layers were separated and the aqueous layer was extracted with $\mathrm{CH}_{2} \mathrm{Cl}_{2}(3 \times 15 \mathrm{~mL})$. The combined organic layers were washed with brine $(20 \mathrm{~mL})$, dried $\left(\mathrm{Na}_{2} \mathrm{SO}_{4}\right)$, and concentrated in vacuo. Flash column chromatography (silica gel, hexanes:EtOAc 5:1) afforded the diyne 16a (567 mg, 85\%) as a colorless oil.

${ }^{1} \mathbf{H}$ NMR (500 MHz, $\left.\mathrm{CDCl}_{3}\right): \delta 5.15$ (ttq, $J=6.9,1.4,1.4 \mathrm{~Hz}, 1 \mathrm{H}, \mathrm{C} 7 H$ ), 5.08 (tqq, $J=6.9,1.2$, $1.2 \mathrm{~Hz}, 1 \mathrm{H}, \mathrm{C} 11 H$ ), 4.31 (br dt, $J=5.8,1.1 \mathrm{~Hz}, 2 \mathrm{H}, \mathrm{C} 2 \mathrm{H}_{2}$ ), 2.98 (dttq, $J=6.8,1.0,1.0,1.0 \mathrm{~Hz}$, $2 \mathrm{H}, \mathrm{C} \equiv \mathrm{CCH}_{2} \mathrm{CH}=\mathrm{C}$ ), 2.08 (br dt, $J=\sim 7, \sim 7 \mathrm{~Hz}, 2 \mathrm{H}, \mathrm{C} 10 H_{2}$ ), 2.00 (br t, $J=7.5 \mathrm{~Hz}, 2 \mathrm{H}, \mathrm{C} \mathrm{H}_{2}$ ), $1.68\left(\mathrm{dt}, J=1.4,1.4 \mathrm{~Hz}, 3 \mathrm{H}, \mathrm{C} 12 \mathrm{ECH} H_{3}\right), 1.62\left(\mathrm{dt}, J=1.4,1.0 \mathrm{~Hz}, 3 \mathrm{H}, \mathrm{C} 8 \mathrm{CH} H_{3}\right)$, and $1.60(\mathrm{dt}, J=$ 1.2, $\left.1.0 \mathrm{~Hz}, 3 \mathrm{H}, \mathrm{C} 12 \mathrm{ZCH}_{3}\right)$.

${ }^{13} \mathrm{C}$ NMR $\left(125 \mathrm{MHz}, \mathrm{CDCl}_{3}\right): \delta 138.9,131.9,124.0,116.9,80.4,74.0,71.0,64.0,51.6,39.5,26.5$, $25.8,18.4,17.8$, and 16.3.

HRMS (CI) $m / z:\left[\mathrm{M}+\mathrm{NH}_{4}\right]^{+}$Calcd for $\mathrm{C}_{15} \mathrm{H}_{24} \mathrm{NO}^{+}$234.1852; Found 234.1835.

IR (neat): 3362, 3053, 2979, 2935, 2866, and $1659 \mathrm{~cm}^{-1}$. 


\section{(E)-8,12-Dimethyltrideca-7,11-dien-2,4-diynal (16b)}

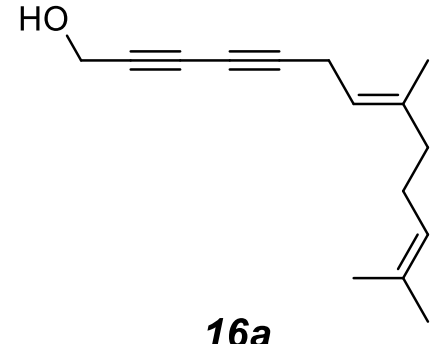

$16 a$

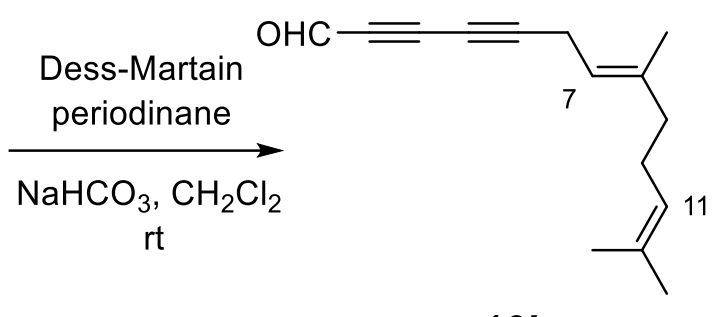

$16 b$

To a stirred solution of alcohol $\mathbf{1 6 a}(800 \mathrm{mg}, 3.7 \mathrm{mmol})$ in $\mathrm{CH}_{2} \mathrm{Cl}_{2}(10 \mathrm{~mL})$ were added $\mathrm{NaHCO}_{3}$ (622 mg, $7.4 \mathrm{mmol})$ and the Dess-Martin periodinane $(1.88 \mathrm{~g}, 4.44 \mathrm{mmol})$. The resulting mixture was stirred at room temperature for $30 \mathrm{~min}$ before it was quenched by the addition of $\mathrm{NaHCO}_{3}$ ( 5 $\mathrm{mL}$, satd aq) and $\mathrm{Na}_{2} \mathrm{SO}_{3}(5 \mathrm{~mL}$, satd aq). The layers were separated and the aqueous layer was extracted with $\mathrm{CH}_{2} \mathrm{Cl}_{2}(3 \times 10 \mathrm{~mL})$. The combined organic layers were washed with brine $(20 \mathrm{~mL})$, dried $\left(\mathrm{Na}_{2} \mathrm{SO}_{4}\right)$, and concentrated in vacuo. Flash column chromatography (silica gel, hexanes:EtOAc 9:1) afforded $\mathbf{1 6 b}(715 \mathrm{mg}, 90 \%)$ as a colorless oil.

${ }^{1} \mathbf{H}$ NMR $\left(500 \mathrm{MHz}, \mathrm{CDCl}_{3}\right): \delta 9.19(\mathrm{t}, J=0.7 \mathrm{~Hz}, 1 \mathrm{H}, \mathrm{CHO}), 5.15(\mathrm{ttq}, J=6.9,1.4,1.4 \mathrm{~Hz}, 1 \mathrm{H}$, $\mathrm{C} 7 H$ ), 5.07 (tqq, $J=6.9,1.4,1.4 \mathrm{~Hz}, 1 \mathrm{H}, \mathrm{C} 11 H$ ), 3.10 (br d, $J=6.9 \mathrm{~Hz}, 2 \mathrm{H}, \mathrm{C} \equiv \mathrm{CCH}_{2} \mathrm{CH}=\mathrm{C}$ ), 2.09 (br dt, $J=\sim 7, \sim 7 \mathrm{~Hz}, 2 \mathrm{H}, \mathrm{C} 10 H_{2}$ ), 2.03 (br t, $J=7.5 \mathrm{~Hz}, 2 \mathrm{H}, \mathrm{C} 9 \mathrm{H}_{2}$ ), 1.69 (dt, $J=1.3,1.3 \mathrm{~Hz}, 3 \mathrm{H}$, $\left.\mathrm{C} 12 \mathrm{ECH}_{3}\right), 1.64$ (br s, 3H, $\mathrm{C} 8 \mathrm{CH}_{3}$ ), and 1.60 (br s, 3H, $\mathrm{C} 12 \mathrm{ZCH}_{3}$ ).

${ }^{13} \mathrm{C}$ NMR (125 MHz, $\left.\mathrm{CDCl}_{3}\right): \delta 176.2,140.1,132.0,123.8,115.3,91.5,80.8,72.7,63.3,39.5$, 26.4, 25.8, 18.9, 17.8, and 16.4.

HRMS (CI) $m / z:\left[\mathrm{M}+\mathrm{NH}_{4}\right]^{+}$Calcd for $\mathrm{C}_{15} \mathrm{H}_{22} \mathrm{NO}^{+} 232.1696$ Found 232.1677.

IR (neat): 2967, 2916, 2856, 2740, 2226, 2130, and $1656 \mathrm{~cm}^{-1}$. 
(E)-8,12-Dimethyltrideca-7,11-dien-2,4-diynoic acid (16c)

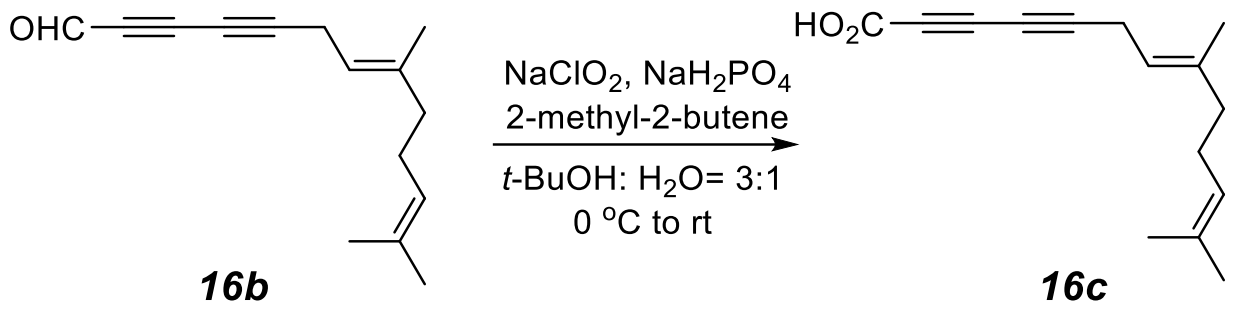

To a stirred solution of aldehyde $\mathbf{1 6 b}(600 \mathrm{mg}, 2.8 \mathrm{mmol})$ in $t-\mathrm{BuOH}(6 \mathrm{~mL})$ and $\mathrm{H}_{2} \mathrm{O}(2 \mathrm{ml})$ were added $\mathrm{NaH}_{2} \mathrm{PO}_{4}$ (672 mg, $\left.5.6 \mathrm{mmol}\right)$, 2-methyl-2-butene (0.6 mL, $\left.5.6 \mathrm{mmol}\right)$, and $\mathrm{NaClO}_{2}(380$ $\mathrm{mg}, 3.36 \mathrm{mmol}$ ) at $0{ }^{\circ} \mathrm{C}$. The reaction mixture was then allowed to warm to room temperature. After being stirred for $1 \mathrm{~h}$ at room temperature the reaction mixture was concentrated under reduced pressure, water was added, and the product was extracted with ethyl acetate. The ethyl acetate layer was washed with water and brine, dried over anhydrous $\mathrm{Na}_{2} \mathrm{SO}_{4}$, filtered, and concentrated under reduced pressure to get the crude acid 16c, which was used for the next reaction without further purification.

${ }^{1} \mathrm{H}$ NMR $\left(500 \mathrm{MHz}, \mathrm{CDCl}_{3}\right): \delta 7.84$ (br s, $\left.1 \mathrm{H}, \mathrm{COOH}\right), 5.15$ (ttq, $\left.J=6.9,1.4,1.4 \mathrm{~Hz}, 1 \mathrm{H}, \mathrm{C} 7 H\right)$, 5.07 (tqq, $J=6.9,1.4,1.4 \mathrm{~Hz}, 1 \mathrm{H}, \mathrm{C} 11 H$ ), 3.10 (br d, $J=6.9 \mathrm{~Hz}, 2 \mathrm{H}, \mathrm{C} \equiv \mathrm{CCH}_{2} \mathrm{CH}=\mathrm{C}$ ), 2.09 (br dt, $J=\sim 7, \sim 7 \mathrm{~Hz}, 2 \mathrm{H}, \mathrm{C} 10 \mathrm{H}_{2}$ ), 2.03 (br t, $J=7.5 \mathrm{~Hz}, 2 \mathrm{H}, \mathrm{C} 9 \mathrm{H}_{2}$ ), 1.69 (dt, $J=1.3,1.3 \mathrm{~Hz}, 3 \mathrm{H}$, $\mathrm{C} 12 \mathrm{ECH}_{3}$ ), 1.64 (br s, 3H, $\mathrm{C} 8 \mathrm{CH}_{3}$ ), and 1.60 (br s, 3H, $\mathrm{C} 12 \mathrm{ZCH}_{3}$ ). 


\section{(E)-N-(3-(Dimethylsilyl)prop-2-yn-1-yl)-8,12-dimethyl- $N$-phenethyltrideca-7,11-dien-2,4-diynamide} (17)

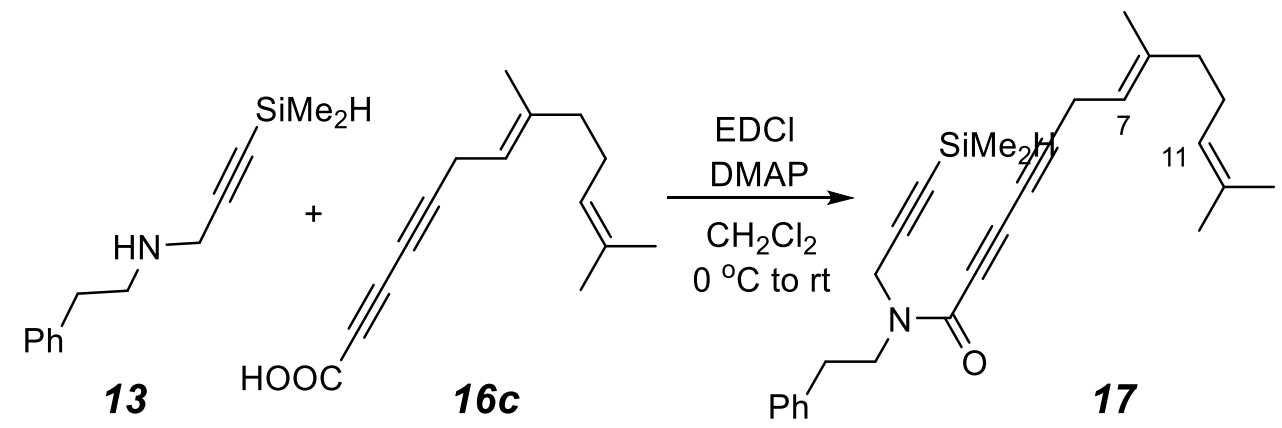

To a stirred solution of the amine $\mathbf{1 3}(434 \mathrm{mg}, 2.0 \mathrm{mmol})$ in $\mathrm{CH}_{2} \mathrm{Cl}_{2}(5 \mathrm{~mL})$ at $0{ }^{\circ} \mathrm{C}$ was added EDCI (460 $\mathrm{mg}, 2.4 \mathrm{mmol})$ followed immediately by a solution of the acid $16 \mathrm{c}(552 \mathrm{mg}, 2.4 \mathrm{mmol})$ and DMAP (4.9 mg, $0.04 \mathrm{mmol})$ in $\mathrm{CH}_{2} \mathrm{Cl}_{2}(5 \mathrm{~mL})$ dropwise over ca. $5 \mathrm{~min}$. The resulting mixture was stirred at room temperature for $1 \mathrm{~h}$ before it was quenched by the addition of $\mathrm{NH}_{4} \mathrm{Cl}(10 \mathrm{~mL}$, satd aq). The layers were separated and the aqueous layer was extracted with $\mathrm{CH}_{2} \mathrm{Cl}_{2}(3 \times 20 \mathrm{~mL})$. The combined organic layers were washed with brine $(20 \mathrm{~mL})$, dried $\left(\mathrm{Na}_{2} \mathrm{SO}_{4}\right)$, and concentrated in vacuo. Flash column chromatography (silica gel, hexanes:EtOAc 10:1) afforded the amide $17(640 \mathrm{mg}, 75 \%)$ as a light yellow oil.

Two rotamers for the tertiary amide (ca. 3:2) were evident in the NMR spectral data:

${ }^{1}$ H NMR $\left(400 \mathrm{MHz}, \mathrm{CDCl}_{3}\right): \delta 7.37-7.28\left(\mathrm{~m}, 2 \mathrm{H}, \mathrm{Ph} H_{m}\right), 7.26-7.19\left(\mathrm{~m}, 3 \mathrm{H}, \mathrm{Ph} H_{o}\right.$ and $\left.\mathrm{Ph} H_{p}\right), 5.20-5.13$ (m, $1 \mathrm{H}, \mathrm{C} 7 H), 5.11-5.05(\mathrm{~m}, 1 \mathrm{H}, \mathrm{C} 11 H), 4.24\left(\mathrm{~d}, J=1.0 \mathrm{~Hz}, 0.8 \mathrm{H}, \mathrm{C} \equiv \mathrm{CCH}_{2} \mathrm{~N}\right.$, minor), $4.23(\mathrm{~d}, J=1.1 \mathrm{~Hz}$, $1.2 \mathrm{H}, \mathrm{C} \equiv \mathrm{CCH}_{2} \mathrm{~N}$, major), $4.12^{+}$[br sept $J=4 \mathrm{~Hz}$, ca. $1 \mathrm{H}, \mathrm{Si} H\left(\mathrm{CH}_{3}\right)_{2}$ ], 4.12- [sept of $\mathrm{t}, J=3.8,1.2 \mathrm{~Hz}$, ca. $1 \mathrm{H}, \mathrm{SiH}\left(\mathrm{CH}_{3}\right)_{2}$ ], 3.88 (nfot, $J_{\text {app }}=7.7 \mathrm{~Hz}, 1.2 \mathrm{H}, \mathrm{NCH}_{2} \mathrm{CH}_{2}$ ), 3.70 (nfot, $J_{\text {app }}=7.7 \mathrm{~Hz}, 0.8 \mathrm{H}, \mathrm{NCH}_{2} \mathrm{CH}_{2}$ ), 3.07 (br t, $J=6.2 \mathrm{~Hz}, 2 \mathrm{H}, \mathrm{C} \equiv \mathrm{CCH}_{2}=\mathrm{C}$ ), 2.97 (nfot, $J_{\text {app }}=7.7 \mathrm{~Hz}, 1.2 \mathrm{H}, \mathrm{NCH}_{2} \mathrm{CH}_{2}$ ), 2.91 (nfot, $J_{\text {app }}=7.7$ $\left.\mathrm{Hz}, 0.8 \mathrm{H}, \mathrm{NCH}_{2} \mathrm{CH}_{2}\right), 2.13-2.05\left(\mathrm{~m}, 2 \mathrm{H}, \mathrm{C} 10 H_{2}\right.$ or $\left.\mathrm{C}_{2} \mathrm{H}_{2}\right), 2.05-1.87$ (m, 2H, C10H or $\left.\mathrm{C}_{2} \mathrm{H}_{2}\right), 1.74(1.69$ (br s, 3H, C12ECH $H_{3}$ ) 1.65 (s, 2H, C8CH $\mathrm{CH}_{3}$, major), 1.64 (s, $1 \mathrm{H}, \mathrm{C} 8 \mathrm{CH}_{3}$, minor), 1.61 (br s, $J=1.3 \mathrm{~Hz}, 3 \mathrm{H}$, $\mathrm{C}_{12} \mathrm{ZCH}_{3}, 0.24\left[\mathrm{~d}, J=3.8 \mathrm{~Hz}, 2.3 \mathrm{H}, \mathrm{SiH}\left(\mathrm{CH}_{3}\right)_{2}\right.$, minor], and 0.24 [d, $J=3.8 \mathrm{~Hz}, 3.7 \mathrm{H}, \mathrm{SiH}\left(\mathrm{CH}_{3}\right)_{2}$, major].

${ }^{13}$ C NMR (101 MHz, $\left.\mathrm{CDCl}_{3}\right): \delta 153.3,153.1,139.74,139.72,138.7,138.0,132.0,129.0,128.92,128.87$, $128.76,126.9,126.7,123.9,115.92,115.86,101.0,100.8,87.6,87.0,86.8,86.7,76.5,76.1,66.3,66.2$, $63.43,63.40,49.9,46.6,40.3,39.51,39.50,35.5,35.1,33.6,26.53,26.50,25.8,18.76,18.74,17.8,16.42$, $16.41,-3.06$, and -3.09 .

HRMS (ESI) $m / z:[\mathrm{M}+\mathrm{H}]^{+}$Calcd for $\mathrm{C}_{28} \mathrm{H}_{36} \mathrm{NOSi}^{+} 430.2561$; Found 430.2551.

IR (neat): 3063, 3027, 2965, 2926, 2857, 2238 (s), 2180 (m), 2147 (m), 2018, and $1658 \mathrm{~cm}^{-1}$. 


\section{(E)-5-(3,7-Dimethylocta-2,6-dien-1-yl)-4-(dimethylsilyl)-6-methoxy-2-phenethylisoindolin- 1-one (18c)}

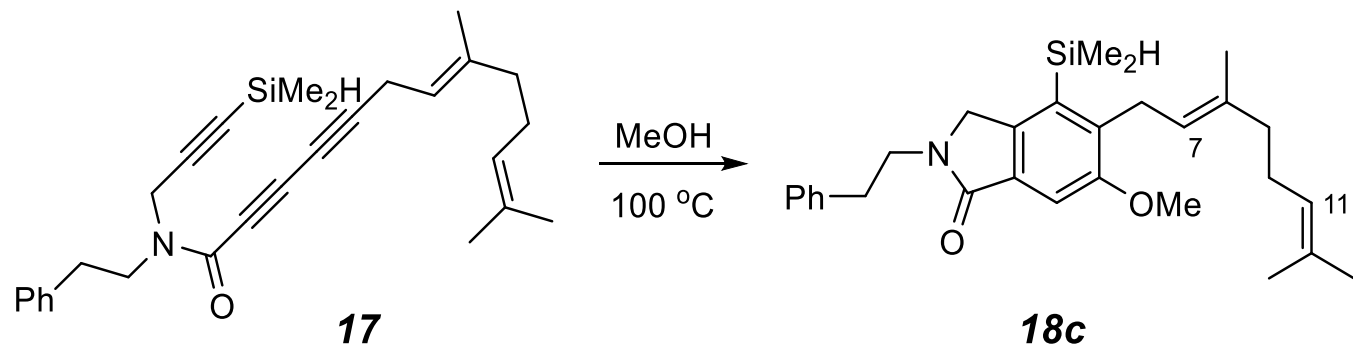

A solution of the amide $17(200 \mathrm{mg}, 0.46 \mathrm{mmol})$ in methanol $(5 \mathrm{~mL})$ was heated in a screw-capped culture tube in an oil bath held at $100^{\circ} \mathrm{C}$. After $2 \mathrm{~h}$ the slightly yellow-colored solution was allowed to cool to room temperature and concentrated in vacuo. Flash column chromatography (silica gel, hexanes:EtOAc 5:1) afforded the lactam 18c (180 $\mathrm{mg}, 84 \%)$ as a light yellow oil.

${ }^{1}$ H NMR $\left(500 \mathrm{MHz}, \mathrm{CDCl}_{3}\right): \delta 7.33(\mathrm{~s}, 1 \mathrm{H}, \mathrm{Ar} H), 7.31-7.26\left(\mathrm{~m}, 2 \mathrm{H}, \mathrm{Ph} H_{m}\right), 7.25\left(\mathrm{~m}, 2 \mathrm{H}, \mathrm{Ph} H_{o}\right)$, 7.21 (tt, $J=7.0,1.4 \mathrm{~Hz}, 1 \mathrm{H}, \mathrm{Ph} H_{p}$ ), 5.05 (tqq, $J=6.7,1.4,1.4 \mathrm{~Hz}, 1 \mathrm{H}, \mathrm{C} 11 H$ ), 4.98 (ttq, $J=6.2$, $1.3,1.3 \mathrm{~Hz}, 1 \mathrm{H}, \mathrm{C} 7 H), 4.50$ [sept, $J=4.0 \mathrm{~Hz}, 1 \mathrm{H}, \mathrm{Si} H\left(\mathrm{CH}_{3}\right)_{2}$ ], 4.17 (s, $\left.2 \mathrm{H}, \mathrm{ArCH}_{2} \mathrm{~N}\right), 3.88-3.84$ (nfot, $2 \mathrm{H}, \mathrm{NCH}_{a} H_{a} \cdot \mathrm{CH}_{\mathrm{b}} \mathrm{H}_{\mathrm{b}} \cdot \mathrm{Ph}$ ), 3.86 (s, $3 \mathrm{H}, \mathrm{OCH}_{3}$ ), 3.50 (dqt, $2 \mathrm{H}, J=6.2,1.1,1.1 \mathrm{~Hz}, 2 \mathrm{H}$, $\mathrm{ArCH}_{2} \mathrm{CH}=\mathrm{C}$ ), 2.99 (nfot, $2 \mathrm{H}, \mathrm{NCH}_{a} \mathrm{H}_{a^{\prime}} \mathrm{CH}_{b} H_{b} \cdot \mathrm{Ph}$ ), 2.05 (br dt, $J=\sim 7, \sim 7 \mathrm{~Hz}, 2 \mathrm{H}, \mathrm{Cl} 0 H_{2}$ ), 1.98 (br t, $J=7.5 \mathrm{~Hz}, 2 \mathrm{H}, \mathrm{C} 9 H_{2}$ ), 1.69 (dt, $\left.J=1.1,1.1 \mathrm{~Hz}, 3 \mathrm{H}, \mathrm{C} 12 \mathrm{EC} H_{3}\right), 1.64$ (dt, $J=1.3,1.3 \mathrm{~Hz}$, $\left.3 \mathrm{H}, \mathrm{C} 8 \mathrm{CH}_{3}\right), 1.57\left(\mathrm{dt}, J=1.2,0.7 \mathrm{~Hz}, 3 \mathrm{H}, \mathrm{C}_{2} 2 \mathrm{ZCH}_{3}\right)$, and 0.35 [d, $\left.J=4.0 \mathrm{~Hz}, 6 \mathrm{H}, \mathrm{SiH}\left(\mathrm{CH}_{3}\right)_{2}\right]$.

${ }^{13}$ C NMR $\left(125 \mathrm{MHz}, \mathrm{CDCl}_{3}\right): \delta 168.9,158.0,140.9,139.4,139.1,135.5,131.8,131.44,131.39$, 128.9, 128.7, 126.6, 124.4, 123.2, 106.1, 55.8, 52.2, 44.3, 39.8, 35.2, 30.1, 26.7, 25.8, 17.8, 16.5, and -2.7 .

HRMS (ESI) $m / z:[\mathrm{M}+\mathrm{H}]^{+}$Calcd for $\mathrm{C}_{29} \mathrm{H}_{40} \mathrm{NO}_{2} \mathrm{Si}^{+} 462.2823$; Found 462.2812 .

IR (neat): 3062, 3026, 2960, 2919, 2855, 2123 (s), 1680, and $1598 \mathrm{~cm}^{-1}$. 


\section{(E)-5-(3,7-Dimethylocta-2,6-dien-1-yl)-6-methoxy-4-(methoxydimethylsilyl)-2-} phenethylisoindolin-1-one (18d)

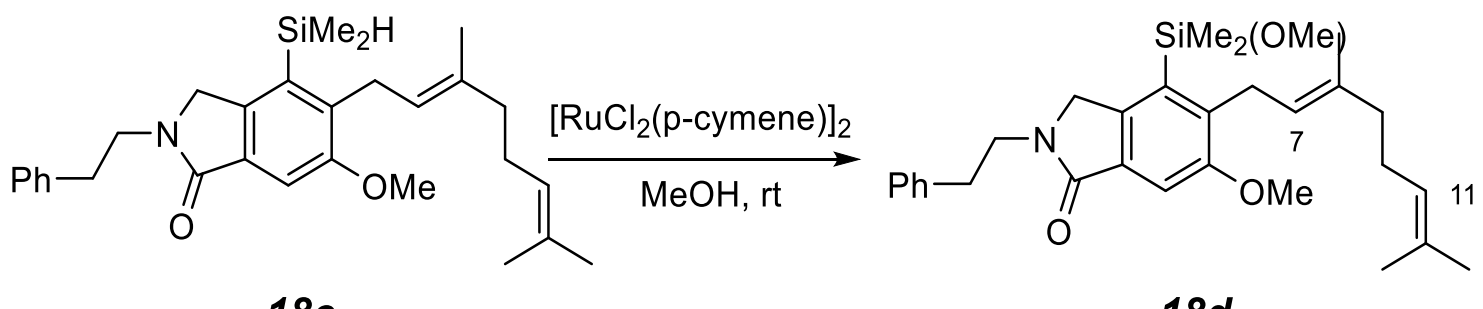

$18 c$

$18 d$

To a stirred solution of $18 \mathrm{c}(50 \mathrm{mg}, 0.11 \mathrm{mmol})$ in $\mathrm{MeOH}(3 \mathrm{~mL})$ was added $\left[\mathrm{RuCl}_{2}(p \text {-cymene })\right]_{2}$ ( $4 \mathrm{mg}, 0.006 \mathrm{mmol}$ ). The resulting mixture was stirred at room temperature for $5 \mathrm{~min}$ before it was quenched by the addition of $\mathrm{NaHCO}_{3}(10 \mathrm{~mL}$, satd aq). The layers were separated and the aqueous layer was extracted with $\mathrm{CH}_{2} \mathrm{Cl}_{2}(3 \times 10 \mathrm{~mL})$. The combined organic layers were washed with brine $(15 \mathrm{~mL})$, dried $\left(\mathrm{Na}_{2} \mathrm{SO}_{4}\right)$, and concentrated in vacuo. Flash column chromatography (silica gel, hexanes:EtOAc 3:1) afforded the methoxysilane 18d (40 mg, 75\%) as a colorless oil.

${ }^{1} \mathbf{H}$ NMR (500 MHz, $\left.\mathrm{CDCl}_{3}\right): \delta 7.35(\mathrm{~s}, 1 \mathrm{H}, \mathrm{Ar} H), 7.29\left(\mathrm{~m}, 2 \mathrm{H}, \mathrm{Ph} H_{m}\right), 7.25-7.19\left(\mathrm{~m}, 3 \mathrm{H}, \mathrm{Ph} H_{o}\right.$ and $\left.\mathrm{Ph} H_{p}\right), 5.05$ (tqq, $\left.J=6.8,1.3,1.3 \mathrm{~Hz}, 1 \mathrm{H}, \mathrm{C} 11 H\right), 4.94$ (ttq, $J=6.0,1.2,1.2 \mathrm{~Hz}, 1 \mathrm{H}, \mathrm{C} 7 H$ ), 4.23 (s, 2H, $\mathrm{ArCH}_{2} \mathrm{~N}$ ), 3.88 (br t, $J=7.5 \mathrm{~Hz}, 2 \mathrm{H}, \mathrm{NCH}_{a} H_{a} \cdot \mathrm{CH}_{\mathrm{b}} \mathrm{H}_{\mathrm{b}}, \mathrm{Ph}$ ), 3.85 (s, 3H, OCH $), 3.46$ (dqt, $2 \mathrm{H}, J=5.8,1.0,1.0 \mathrm{~Hz}, 2 \mathrm{H}, \mathrm{ArCH}_{2} \mathrm{CH}=\mathrm{C}$ ), 3.40 [s, 3H, $\left.\mathrm{Si}\left(\mathrm{CH}_{3}\right)_{2}\left(\mathrm{OCH}_{3}\right)\right], 3.00$ (br t, $J=7.6$ $\mathrm{Hz}, 2 \mathrm{H}, \mathrm{NCH}_{\mathrm{a}} \mathrm{H}_{\mathrm{a}} \mathrm{CH}_{b} \mathrm{H}_{b}$, $\mathrm{Ph}$ ), 2.04 (br dt, $J=\sim 7, \sim 7 \mathrm{~Hz}, 2 \mathrm{H}, \mathrm{C} 10 H_{2}$ ), 1.98 (br t, $J=7.5 \mathrm{~Hz}, 2 \mathrm{H}$, $\left.\mathrm{C} \mathrm{H}_{2}\right), 1.73\left(\mathrm{dt}, J=1.1,1.1 \mathrm{~Hz}, 3 \mathrm{H}, \mathrm{C} 12 \mathrm{ECH}_{3}\right), 1.64(\mathrm{dt}, J=1.3,1.3 \mathrm{~Hz}, 3 \mathrm{H}, \mathrm{C} 8 \mathrm{CH}$ ) $), 1.57$ (br d, $\left.J=1.3 \mathrm{~Hz}, 3 \mathrm{H}, \mathrm{C} 12 \mathrm{ZCH}_{3}\right)$, and $0.39\left[\mathrm{~s}, 6 \mathrm{H}, \mathrm{Si}\left(\mathrm{CH}_{3}\right)_{2}\left(\mathrm{OCH}_{3}\right)\right]$.

${ }^{13} \mathrm{C}$ NMR (125 MHz, $\left.\mathrm{CDCl}_{3}\right): \delta$ 168.7, 158.0, 140.4, 139.3, 139.3, 135.4, 132.5, 131.8, 131.4, $128.9,128.7,126.6,124.4,123.5,106.2,55.8,52.9,50.3,44.2,39.7,35.2,29.9,26.7,25.8,17.8$, 16.5 , and 0.5 .

HRMS (ESI) $m / z$ : $[\mathrm{M}+\mathrm{H}]^{+}$Calcd for $\mathrm{C}_{30} \mathrm{H}_{42} \mathrm{NO}_{3} \mathrm{Si}^{+}$492.2928; Found 492.2899.

IR (neat): 2927, 2932, 2910, and $1673 \mathrm{~cm}^{-1}$. 


\section{(E)-5-(3,7-Dimethylocta-2,6-dien-1-yl)-4-(hydroxydimethylsilyl)-6-methoxy-2- phenethylisoindolin-1-one (18e)}

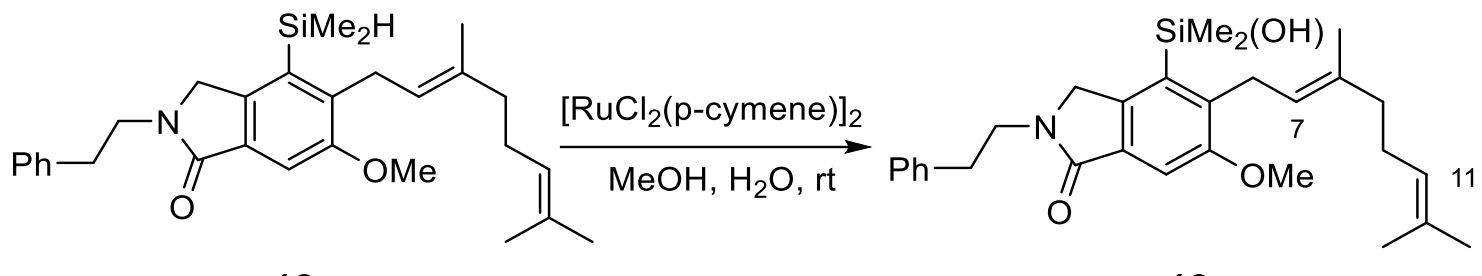

$18 c$

$18 e$

To a stirred solution of $\mathbf{1 8 c}(80 \mathrm{mg}, 0.17 \mathrm{mmol})$ in $\mathrm{MeOH}(3 \mathrm{~mL})$ and $\mathrm{H}_{2} \mathrm{O}(1 \mathrm{~mL})$ was added $\left[\mathrm{RuCl}_{2}(p \text {-cymene })\right]_{2}(5 \mathrm{mg}, 0.009 \mathrm{mmol})$. The resulting mixture was stirred at room temperature for $30 \mathrm{~min}$ before it was quenched by the addition of $\mathrm{NaHCO}_{3}(10 \mathrm{~mL}$, satd aq). The layers were separated and the aqueous layer was extracted with $\mathrm{CH}_{2} \mathrm{Cl}_{2}(3 \times 10 \mathrm{~mL})$. The combined organic layers were washed with brine $(15 \mathrm{~mL})$, dried $\left(\mathrm{Na}_{2} \mathrm{SO}_{4}\right)$, and concentrated in vacuo. Flash column chromatography (silica gel, hexanes:EtOAc 3:1) afforded the silanol 18e (72 $\mathrm{mg}, 87 \%)$ as a colorless oil.

${ }^{1} \mathbf{H}$ NMR (500 MHz, $\left.\mathrm{CDCl}_{3}\right): \delta 7.33(\mathrm{~s}, 1 \mathrm{H}, \mathrm{Ar} H), 7.26\left(\mathrm{~m}, 2 \mathrm{H}, \mathrm{Ph} H_{m}\right), 7.22-7.18\left(\mathrm{~m}, 3 \mathrm{H}, \mathrm{Ph} H_{o}\right.$ and $\left.\mathrm{Ph} H_{p}\right), 5.04$ (tqq, $J=6.7,1.3,1.3 \mathrm{~Hz}, 1 \mathrm{H}, \mathrm{C} 11 H$ ), 4.97 (ttq, $J=5.9,1.2,1.2 \mathrm{~Hz}, 1 \mathrm{H}, \mathrm{C} 7 H$ ), $4.33\left(\mathrm{~s}, 2 \mathrm{H}, \mathrm{ArCH}_{2} \mathrm{~N}\right), 3.83$ (s, 3H, $\left.\mathrm{OCH}_{3}\right), 3.79$ (br t, $\left.J=7.5 \mathrm{~Hz}, 2 \mathrm{H}, \mathrm{NCH}_{a} H_{a} \cdot \mathrm{CH}_{\mathrm{b}} \mathrm{H}_{\mathrm{b}} \mathrm{Ph}\right), 3.48$ (dqt, $2 \mathrm{H}, J=5.9,1.0,1.0 \mathrm{~Hz}, 2 \mathrm{H}, \mathrm{ArCH}_{2} \mathrm{CH}=\mathrm{C}$ ), 2.99 (br t, $J=7.6 \mathrm{~Hz}, 2 \mathrm{H}, \mathrm{NCH}_{\mathrm{a}} \mathrm{H}_{\mathrm{a}} \mathrm{CH}_{b} H_{b}, \mathrm{Ph}$ ), 2.05 (br dt, $J=\sim 7, \sim 7 \mathrm{~Hz}, 2 \mathrm{H}, \mathrm{C} 10 \mathrm{H}_{2}$ ), 1.98 (br t, $J=7.5 \mathrm{~Hz}, 2 \mathrm{H}, \mathrm{C} 9 \mathrm{H}_{2}$ ), 1.73 (dt, $J=1.1,1.1 \mathrm{~Hz}$, $\left.3 \mathrm{H}, \mathrm{C} 12 \mathrm{ECH}_{3}\right), 1.64$ (dt, $\left.J=1.3,1.3 \mathrm{~Hz}, 3 \mathrm{H}, \mathrm{C} 8 \mathrm{CH}_{3}\right), 1.57$ (br d, $J=1.3 \mathrm{~Hz}, 3 \mathrm{H}, \mathrm{C} 12 \mathrm{ZCH}$ ), and $0.43\left[\mathrm{~s}, 6 \mathrm{H}, \mathrm{SiH}\left(\mathrm{CH}_{3}\right)_{2}\right]$.

${ }^{13} \mathrm{C}$ NMR $\left(125 \mathrm{MHz}, \mathrm{CDCl}_{3}\right): \delta 168.7,158.0,140.0,139.1,138.9,136.1,133.7,131.8,131.6$, 128.9, 128.7, 126.6, 124.3, 123.7, 106.1, 55.8, 52.9, 44.2, 39.7, 35.1, 30.0, 26.7, 25.8, 17.8, 16.6, and 3.1.

HRMS (ESI) $m / z:[\mathrm{M}+\mathrm{H}]^{+}$Calcd for $\mathrm{C}_{29} \mathrm{H}_{40} \mathrm{NO}_{3} \mathrm{Si}^{+} 478.2772$; Found 478.2751.

IR (neat): 2984, 2942, 2909, and $1650 \mathrm{~cm}^{-1}$. 


\section{Isohericerin (1)}

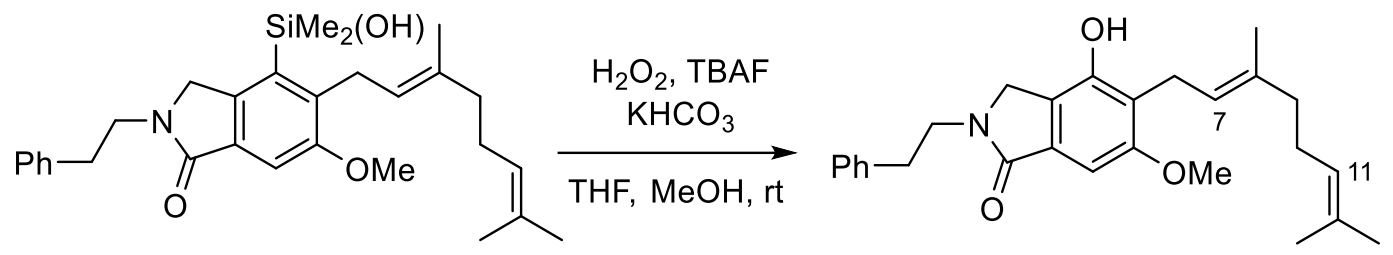

$18 e$

isohericerin (1)

To a stirred solution of $\mathbf{1 8 e}(50 \mathrm{mg}, 0.1 \mathrm{mmol})$ in $\mathrm{MeOH}(2 \mathrm{~mL})$ and THF $(2 \mathrm{~mL})$ were added $\mathrm{KHCO}_{3}(5.2 \mathrm{mg}, 0.05 \mathrm{mmol}), \mathrm{H}_{2} \mathrm{O}_{2}\left(30 \mathrm{wt} . \%\right.$ in $\left.\mathrm{H}_{2} \mathrm{O}, 71 \mu \mathrm{L}, 0.6 \mathrm{mmol}\right)$ and TBAF (1.0 M in THF, $10 \mu \mathrm{L}, 0.01 \mathrm{mmol})$. The resulting mixture was stirred at room temperature for $2 \mathrm{~h}$ before it was quenched by the addition of $\mathrm{NaHCO}_{3}(10 \mathrm{~mL}$, satd aq). The layers were separated and the aqueous layer was extracted with $\mathrm{CH}_{2} \mathrm{Cl}_{2}(3 \times 10 \mathrm{~mL})$. The combined organic layers were washed with brine (20 mL), dried $\left(\mathrm{Na}_{2} \mathrm{SO}_{4}\right)$, and concentrated in vacuo. Flash column chromatography (silica gel, hexanes:EtOAc 4:1) afforded isohericerin (1) (37 mg, 82\%) as a white solid. Crystals grown by vapor diffusion $\left(\mathrm{CH}_{2} \mathrm{Cl}_{2}\right.$ /pentane) gave the $\mathrm{mp}$ data below, which matched well the literature reported value.

${ }^{1} \mathbf{H}$ NMR (500 MHz, $\mathrm{CDCl}_{3}$ ): $\delta 7.26$ (nfom, 2H, $\mathrm{Ph} H_{m}$ ), 7.21 (nfom, 2H, $\mathrm{PhH}$ ), 7.19 (tt, $J=7.1$, $1.4 \mathrm{~Hz}, 1 \mathrm{H}, \mathrm{Ph} H_{p}$ ), 6.96 (s, 1H, ArH), 6.31 (br s, 1H, ArOH), 5.24 (dqt, J = 7.1, 1.3, 1.3 Hz, 1H, $\mathrm{C} 7 \mathrm{H}$ ), 5.03 (dqq, $J=6.8,1.4,1.4 \mathrm{~Hz}, 1 \mathrm{H}, \mathrm{C} 7 \mathrm{H}$ ), 4.17 (s, 2H, $\mathrm{ArCH}_{2} \mathrm{~N}$ ), 3.84 (nfot, 2H, $\mathrm{NCH}_{a} H_{a} \cdot \mathrm{CH}_{\mathrm{b}} \mathrm{H}_{\mathrm{b}} \mathrm{Ph}$ ), $3.83\left(\mathrm{~s}, 3 \mathrm{H}, \mathrm{OCH}_{3}\right), 3.49$ (dqt, $2 \mathrm{H}, J=7.2,1.0,1.0 \mathrm{~Hz}, 2 \mathrm{H}, \mathrm{ArCH} \mathrm{CH}_{2} \mathrm{C}$ ), 2.97 (nfot, $2 \mathrm{H}, \mathrm{NCH}_{\mathrm{a}} \mathrm{H}_{\mathrm{a}} \mathrm{CH}_{b} \mathrm{H}_{b} \mathrm{Ph}$ ), 2.10 (br dt, $J=\sim 7, \sim 7 \mathrm{~Hz}, 2 \mathrm{H}, \mathrm{C} 10 H_{2}$ ), 2.06 (br t, $J=\sim 7 \mathrm{~Hz}$, $\left.2 \mathrm{H}, \mathrm{C} 9 \mathrm{H}_{2}\right), 1.81\left(\mathrm{dt}, J=1.1,1.1 \mathrm{~Hz}, 3 \mathrm{H}, \mathrm{C} 12 \mathrm{ECH}_{3}\right), 1.66(\mathrm{dt}, J=1.4,1.4 \mathrm{~Hz}, 3 \mathrm{H}, \mathrm{C} 8 \mathrm{CH}$ ), and 1.58 (br d, $J=1.4 \mathrm{~Hz}, 3 \mathrm{H}, \mathrm{C}^{2} 2 \mathrm{ZCH}_{3}$ ).

${ }^{13} \mathrm{C}$ NMR $\left(125 \mathrm{MHz}, \mathrm{CDCl}_{3}\right): \delta 169.1,158.6,150.7,139.5,138.9,132.30,132.29,128.8,128.7$, $126.6,123.8,121.4,121.3,118.6,97.7,56.2$, 48.3, 44.4, 39.8, 35.0, 26.4, 25.8, 22.9, 17.8, and 16.3 .

HRMS (ESI) $m / z:[\mathrm{M}+\mathrm{H}]^{+}$Calcd for $\mathrm{C}_{27} \mathrm{H}_{34} \mathrm{NO}_{3}{ }^{+} 420.2533$; Found 420.2515.

IR (neat): 3020 (br), 2924, 2854, 1655, and $1595 \mathrm{~cm}^{-1}$.

mp: $158-160{ }^{\circ} \mathrm{C}\left(\right.$ lit. $\left.{ }^{5} 157-158.5^{\circ} \mathrm{C}\right)$. 


\section{(E)-2-(4-Hydroxy-4-methylpent-2-en-1-yl)-5-methoxy-2-methyl-8-phenethyl-3,4,8,9- tetrahydropyrano[2,3-e]isoindol-7(2H)-one (21)}

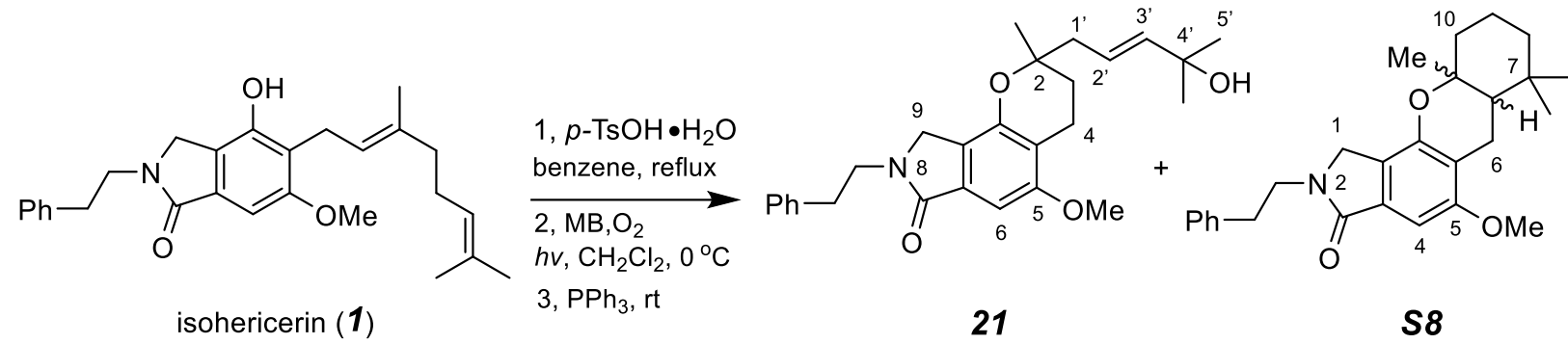

To a stirred solution of isohericerin (1) $(50 \mathrm{mg}, 0.143 \mathrm{mmol})$ in benzene $(5 \mathrm{~mL})$ was added $p$ toluenesulfonic acid monohydrate $(3 \mathrm{mg}, 0.0143 \mathrm{mmol})$. The resulting mixture was refluxed for 30 min while being stirred before it was cooled to room temperature and then quenched by the addition of $\mathrm{NaHCO}_{3}(10 \mathrm{~mL}$, satd aq). The layers were separated and the aqueous layer was extracted with $\mathrm{CH}_{2} \mathrm{Cl}_{2}(3 \times 5 \mathrm{~mL})$. The combined organic layers were washed with brine $(10 \mathrm{~mL})$, dried $\left(\mathrm{Na}_{2} \mathrm{SO}_{4}\right)$, and concentrated in vacuo. Flash column chromatography (silica gel, hexanes:EtOAc 1:1) afforded a mixture of coeluting compounds (45 mg, 90\%) as a colorless oil. This mixture, whose ${ }^{1} \mathrm{H}$ NMR spectrum suggested the presence of $\mathbf{2 0}$ and two diastereomers of $\mathbf{S 8}$, was used in the next reaction without further purification.

To a stirred solution of the mixture containing $\mathbf{2 0}$ in the estimated amount of $30 \mathrm{mg}(0.07 \mathrm{mmol})$ in $\mathrm{CH}_{2} \mathrm{Cl}_{2}(10 \mathrm{~mL})$ was added methylene blue $(1 \mathrm{mg}, 0.007 \mathrm{mmol})$. The mixture was purged several times with $\mathrm{O}_{2}$ (balloon), cooled to $0{ }^{\circ} \mathrm{C}$, and irradiated with a $100 \mathrm{~W}$ lamp for $30 \mathrm{~min}$, stirring under the oxygen atmosphere. The mixture was warmed to room temperature and the solvent was evaporated to leave an oily residue ( $31 \mathrm{mg}, 0.07 \mathrm{mmol}$ ). This was dissolved in $\mathrm{CH}_{2} \mathrm{Cl}_{2}$ $(5 \mathrm{~mL})$ and triphenylphosphine $(18 \mathrm{mg}, 0.07 \mathrm{mmol})$ was added. The resulting mixture was stirred at room temperature for $5 \mathrm{~min}$ and $\mathrm{NaHCO}_{3}(10 \mathrm{~mL}$, satd aq) was added. The layers were separated and the aqueous layer was extracted with $\mathrm{CH}_{2} \mathrm{Cl}_{2}(3 \times 5 \mathrm{~mL})$. The combined organic layers were washed with brine $(10 \mathrm{~mL})$, dried $\left(\mathrm{Na}_{2} \mathrm{SO}_{4}\right)$, and concentrated in vacuo. Flash column chromatography (silica gel, hexanes:EtOAc 1:1) afforded the diastereomeric mixture of $\mathbf{S 8}$ followed by the slower eluting alcohol 21 (15 mg, 30\%, 3 steps from isohericerin) as a colorless oil.

\section{(E)-2-(4-Hydroxy-4-methylpent-2-en-1-yl)-5-methoxy-2-methyl-8-phenethyl-3,4,8,9- tetrahydropyrano[2,3-e]isoindol-7(2H)-one (21)}

${ }^{1} \mathbf{H}$ NMR $\left(500 \mathrm{MHz}, \mathrm{CDCl}_{3}\right): \delta$ 7.30-7.27 (m, 2H, $\left.\mathrm{Ph} H_{m}\right), 7.26-7.23\left(\mathrm{~m}, 2 \mathrm{H}, \mathrm{Ph} H_{o}\right), 7.20$ (tt, $J=$ 7.1, $1.4 \mathrm{~Hz}, 1 \mathrm{H}, \mathrm{Ph} H_{p}$ ), 6.88 (s, $1 \mathrm{H}, \mathrm{C} 6 H$ ), 5.70-5.65 (overlapping nfom's, 2H, C2 ' $H+\mathrm{C} 3$ ' $H$ ), 4.15 (d, $J=16.8 \mathrm{~Hz}, 1 \mathrm{H}, \mathrm{C} 9 \mathrm{H}_{a} \mathrm{Hb}$ ), $4.14\left(\mathrm{~d}, J=16.7 \mathrm{~Hz}, 1 \mathrm{H}, \mathrm{C} \mathrm{H}_{a} H_{b}\right), 3.87$ (s, $\left.3 \mathrm{H}, \mathrm{OCH}_{3}\right), 3.83$ (nfot, $2 \mathrm{H}, \mathrm{NCH}_{a} \mathrm{H}_{a} \cdot \mathrm{CH} b \mathrm{Hb}_{b} \mathrm{Ph}$ ), 2.97 (nfot, $2 \mathrm{H}, \mathrm{NCH}_{a} \mathrm{H}_{\mathrm{a}} \mathrm{CH}_{b} \mathrm{H}_{b} \cdot \mathrm{Ph}$ ), 2.70 (ddd, $J=17.8,6.5,6.5 \mathrm{~Hz}, 1 \mathrm{H}$, $\mathrm{C} 4 H_{a} \mathrm{H}_{\mathrm{b}}$ ), 2.66 (ddd, $J=17.9,7.2,6.5 \mathrm{~Hz}, 1 \mathrm{H}, \mathrm{C}_{4} \mathrm{H}_{\mathrm{a}} H_{b}$ ), 2.36-2.28 (overlapping nfom's, $2 \mathrm{H}$, $\mathrm{Cl}^{\prime} H_{2}$ ), 1.83 (ddd, $\left.J=13.8,7.1,7.1 \mathrm{~Hz}, 1 \mathrm{H}, \mathrm{C} 3 \mathrm{H}_{a} \mathrm{Hb}_{\mathrm{b}}\right), 1.76$ (ddd, $J=13.6,6.8,6.8 \mathrm{~Hz}, 1 \mathrm{H}$, 
$\left.\mathrm{C}^{3} \mathrm{H}_{\mathrm{a}} \mathrm{H}_{b}\right), 1.320$ [s, 3H, C4 $\left.{ }^{\prime}\left(\mathrm{CH}_{3}\right) \mathrm{CH}_{3}{ }^{\prime}\right], 1.317$ [s, 3H, C4 $\left.{ }^{\prime}\left(\mathrm{CH}_{3}\right) \mathrm{CH}_{3}{ }^{\prime}\right]$, and $1.26\left(\mathrm{br} \mathrm{s}, 3 \mathrm{H}, \mathrm{C} 2 \mathrm{CH}_{3}\right)$.

${ }^{13} \mathrm{C}$ NMR (125 MHz, $\left.\mathrm{CDCl}_{3}\right): \delta 169.1,158.6,149.2,142.2,139.0,132.3,128.87,128.7,126.6$, 122.1, 121.4, 113.6, 96.0, 76.4, 70.9, 56.0, 48.1, 44.3, 42.2, 35.1, 30.2, 30.1(x2), 24.4, and 17.4.

HRMS (ESI) $m / z:[\mathrm{M}+\mathrm{H}]^{+}$Calcd for $\mathrm{C}_{27} \mathrm{H}_{34} \mathrm{NO}_{4}{ }^{+}$436.2482; Found 436.2465.

IR (neat): 3396, 3060, 3020, 2966, 2928, 2860, 1672, and $1608 \mathrm{~cm}^{-1}$.

$( \pm)-(6 \mathrm{a} R, 10 \mathrm{a} R)-5-M e t h o x y-7,7,10 \mathrm{a}-t$ rimethyl-2-phenethyl-1,2,6a,7,8,9,10,10aoctahydrochromeno[2,3-e]isoindol-3(6H)-one (trans-S8)

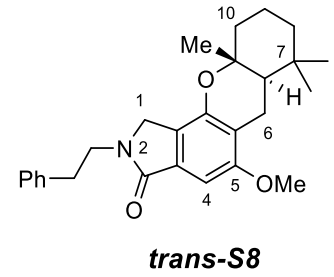

${ }^{1} \mathbf{H}$ NMR $\left(500 \mathrm{MHz} \mathrm{CDCl}_{3}\right): \delta 7.29$ (nfot, $\left.J_{\text {app }}=7.7 \mathrm{~Hz}, 2 \mathrm{H}, \mathrm{Ph} H_{m}\right), 7.24\left(\right.$ nfod, $J_{\text {app }}=7.3 \mathrm{~Hz}, 2 \mathrm{H}$, $\mathrm{Ph} H_{o}$ ), 7.21 (tt, $\left.J=7.2,1.4 \mathrm{~Hz}, 1 \mathrm{H}, \mathrm{Ph} H_{p}\right), 6.88$ (s, $\left.1 \mathrm{H}, \mathrm{C} 4 H\right), 4.15\left(\mathrm{~d}, J=17.0 \mathrm{~Hz}, 1 \mathrm{H}, \mathrm{C} 1 H_{a} \mathrm{H}_{\mathrm{b}}\right)$, $4.14\left(\mathrm{~d}, J=17.0 \mathrm{~Hz}, 1 \mathrm{H}, \mathrm{C} 1 \mathrm{H}_{\mathrm{a}} H_{b}\right.$ ), 3.89 (s, 3H, OCH ), 3.83 (nfot, 2H, NCH $H_{a} \mathrm{CH}_{\mathrm{b}} \mathrm{H}_{\mathrm{b}} \cdot \mathrm{Ph}$ ), 2.98 (nfot, $2 \mathrm{H}, \mathrm{NCH}_{\mathrm{a}} \mathrm{H}_{\mathrm{a}} \mathrm{CH}_{b} \mathrm{H}_{b} \cdot \mathrm{Ph}$ ), 2.81 (dd, $J=18.0,5.0 \mathrm{~Hz}, 1 \mathrm{H}, \mathrm{C} 6 H_{a} \mathrm{H}_{\mathrm{b}}$ ), 2.31 (dd, $J=17.6,13.3$ $\left.\mathrm{Hz}, 1 \mathrm{H}, \mathrm{C} 6 \mathrm{H}_{\mathrm{a}} H_{b}\right), 1.64(\mathrm{dd}, J=13.3,4.8 \mathrm{~Hz}, 1 \mathrm{H}, \mathrm{C} 6 \mathrm{a} H$ ), br overlapping multiplets (aka envelope)

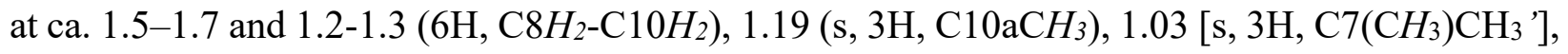
and $0.93\left[3 \mathrm{H}, \mathrm{C} 7\left(\mathrm{CH}_{3}\right) \mathrm{CH}_{3}{ }^{\prime}\right]$.

${ }^{13} \mathrm{C}$ NMR (125 MHz, $\left.\mathrm{CDCl}_{3}\right): \delta 169.1,158.6,148.7,139.0,132.1,128.9,128.7,126.6,122.0$, 115.1, 96.0, 77.8, 56.0, 48.2, 47.7, 44.3, 41.7, 39.9, 35.1, 33.7, 32.3, 20.7, 20.0, 19.9, and 18.8.

HRMS (ESI) $m / z:[\mathrm{M}+\mathrm{H}]^{+}$Calcd for $\mathrm{C}_{27} \mathrm{H}_{34} \mathrm{NO}_{3}{ }^{+} 420.2533$; Found 420.2513.

IR (neat): 2929, 2874, 1688, and $1607 \mathrm{~cm}^{-1}$.

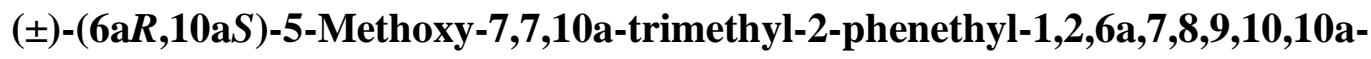
octahydrochromeno $[2,3-e]$ isoindol-3(6H)-one (cis-S8)

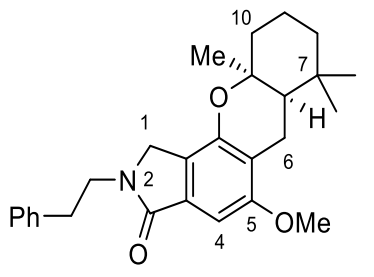

cis-S8

${ }^{1} \mathbf{H}$ NMR $\left(500 \mathrm{MHz}, \mathrm{CDCl}_{3}\right.$ ): $\delta 7.29$ (nfot, $\left.J_{\text {app }}=7.3 \mathrm{~Hz}, 2 \mathrm{H}, \mathrm{Ph} H_{m}\right), 7.25\left(\mathrm{nfod}, J_{\text {app }}=\sim 8 \mathrm{~Hz}, 2 \mathrm{H}\right.$, $\left.\mathrm{Ph} H_{o}\right), 7.22$ (tt, $\left.J=7.1,1.4 \mathrm{~Hz}, 1 \mathrm{H}, \mathrm{Ph} H_{p}\right), 6.87$ (s, 1H, C4H), 4.14 (s, 2H, C1H $), 3.89$ (s, 3H, $\mathrm{OCH}_{3}$ ), 3.81 (nfot, 2H, $\mathrm{NCH}_{a} H_{a} \cdot \mathrm{CH}_{\mathrm{b}} \mathrm{H}_{\mathrm{b}}$, $\mathrm{Ph}$ ), 2.98 (nfot, $2 \mathrm{H}, \mathrm{NCH}_{a} \mathrm{H}_{\mathrm{a}} \cdot \mathrm{CH}_{b} H_{b}, \mathrm{Ph}$ ), 2.83 (ddd, $J=$ 
18.6, 0.8, $\left.0.8 \mathrm{~Hz}, 1 \mathrm{H}, \mathrm{C} 6 H_{a} \mathrm{H}_{\mathrm{b}}\right), 2.71\left(\mathrm{dd}, J=18.6,8.0 \mathrm{~Hz}, 1 \mathrm{H}, \mathrm{C}_{6} \mathrm{H}_{\mathrm{a}} H_{b}\right.$ ), 2.01 (nfom includes an ca. $13 \mathrm{~Hz}$ doublet, $1 \mathrm{H}), 1.8$ (nfom, $1 \mathrm{H}), 1.50(\mathrm{dd}, J=8.0,0.9 \mathrm{~Hz}, 1 \mathrm{H}, \mathrm{C} 6 \mathrm{a} H), 1.55-1.4$ (overlapped m's, ca. 3H), 1.27 (nfom, 1H), 1.18 (s, 3H, C10aCH3), 0.97 (s, 3H, C7( $\left.\mathrm{CH}_{3}\right) \mathrm{CH}_{3}$ '), and 0.58 (br s, $\left.3 \mathrm{H}, \mathrm{C} 7\left(\mathrm{CH}_{3}\right) \mathrm{CH}_{3}{ }^{\prime}\right)$.

${ }^{13} \mathrm{C}$ NMR $\left(125 \mathrm{MHz}, \mathrm{CDCl}_{3}\right): \delta 169.2,158.2,149.9,139.0,131.7,128.9,128.7,126.6,122.0$, 114.6, 96.0, 75.9, 56.0, 48.1, 44.4, 43.8, 41.7, 39.4, 35.1, 34.2, 32.4, 27.1, 21.4, 19.1, and 18.1.

HRMS (ESI) $m / z:[\mathrm{M}+\mathrm{H}]^{+}$Calcd for $\mathrm{C}_{27} \mathrm{H}_{34} \mathrm{NO}_{3}{ }^{+}$420.2533; Found 420.2512.

IR (neat): 2972, 2855, 1692, and $1610 \mathrm{~cm}^{-1}$.

\section{Assignment of the stereochemical relationship in trans-S8 vs. cis-S8.}

There are several features that can be seen in the 1D ${ }^{1} \mathrm{H}$ NMR spectral data of these diastereomers to provide the indicated assignment, but one definitively diagnostic trait is the presence of a highly shielded singlet for one of the two methyl groups in the cis-isomer. A significantly populated conformation is one in which the Me (green) trans to the angular hydrogen atom lies within the concave cavity of the bicyclic oxadecalin, where it is anisotropically shielded by the nearby arene ring. There are numerous published examples that demonstrate this; two are shown below (an Xray diffraction structure of the first confirms its assignment).

\begin{tabular}{|c|c|c|}
\hline $\begin{array}{c}\text { ref } \\
6\end{array}$ & $\begin{array}{l}\text { "1 H NMR }\left(\mathbf{C D C l}_{3}, 400 \text { MHz) } \delta 6.34(\mathrm{~s}, 1 \mathrm{H}), 2.62(\mathrm{dd}, \mathrm{J}=16.8,4.8 \mathrm{~Hz},\right. \\
1 \mathrm{H}), 2.57(\mathrm{~s}, 3 \mathrm{H}), 2.22(\mathrm{dd}, \mathrm{J}=16.8,13.3 \mathrm{~Hz}, 1 \mathrm{H}), 2.01-1.94(\mathrm{~m}, 1 \mathrm{H}), \\
1.74(\mathrm{~s}, 3 \mathrm{H}), 1.70(\mathrm{~s}, 3 \mathrm{H}), 1.68-1.64(\mathrm{~m}, 1 \mathrm{H}), 1.60(\mathrm{dd}, \mathrm{J}=13.4,4.8 \mathrm{~Hz}, \\
1 \mathrm{H}), 1.60-1.55(\mathrm{~m}, 2 \mathrm{H}), 1.54-1.47(\mathrm{~m}, 1 \mathrm{H}), 1.37-1.28(\mathrm{~m}, 1 \mathrm{H}), 1.21(\mathrm{~s}, \\
3 \mathrm{H}), 1.03(\mathrm{~s}, 3 \mathrm{H}), 0.93(\mathrm{~s}, 3 \mathrm{H}) . "\end{array}$ & \\
\hline
\end{tabular}

\begin{tabular}{|c|l|}
\hline ref & “1H NMR $\left(\mathbf{C D C l}_{3}, \mathbf{5 0 0} \mathbf{M H z}\right) \delta: 7.05(\mathrm{dd}, \mathrm{J}=7.6,7.6 \mathrm{~Hz}, 1 \mathrm{H}), 7.04(\mathrm{~d}$, \\
7 & $\mathrm{~J}=7.6 \mathrm{~Hz}, 1 \mathrm{H}), 6.81(\mathrm{dd}, \mathrm{J}=7.3,7.3 \mathrm{~Hz}, 1 \mathrm{H}), 6.75(\mathrm{~d}, \mathrm{~J}=8 \mathrm{~Hz}, 1 \mathrm{H})$, \\
& $3.04(\mathrm{dd}, \mathrm{J}=17.6,8.0 \mathrm{~Hz}, 1 \mathrm{H}), 2.77(\mathrm{~d}, \mathrm{~J}=17.6 \mathrm{~Hz}, 1 \mathrm{H}), 2.04(\mathrm{br} \mathrm{d}, \mathrm{J}=$ \\
& $\begin{array}{l}\text { 14.6 Hz, } 1 \mathrm{H}), 1.86(\mathrm{~m}, 1 \mathrm{H}), 1.59-1.36(\mathrm{~m}, 4 \mathrm{H}), 1.27(\mathrm{t}, \mathrm{J}=11.9 \mathrm{~Hz} \text { or } \\
\mathrm{dd}, \mathrm{J}=26.0,15.5 \mathrm{~Hz}, 1 \mathrm{H}), 1.21(\mathrm{~s}, 3 \mathrm{H}), 0.97(\mathrm{~s}, 3 \mathrm{H}), 0.65(\mathrm{~s}, 3 \mathrm{H}) . ”\end{array}$ \\
\hline
\end{tabular}




\section{Erinacerin A (2)}

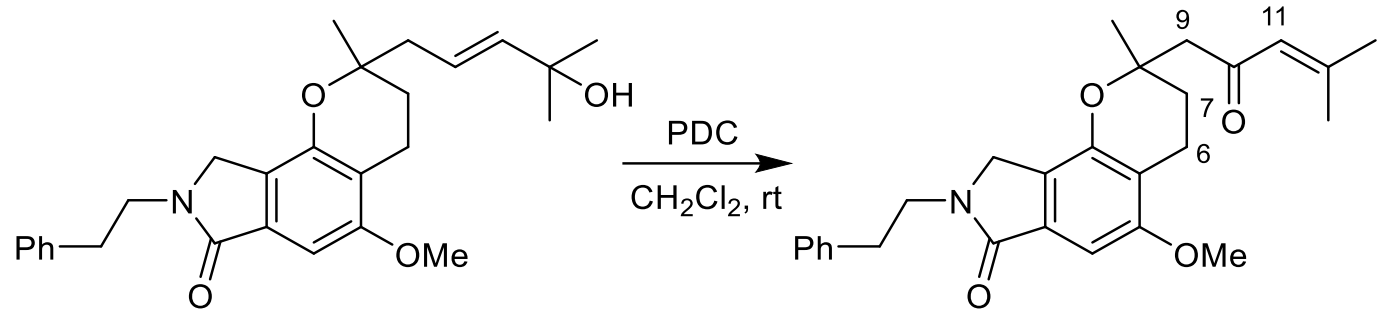

21

erinacerin A (2)

To a stirred solution of $21(10 \mathrm{mg}, 0.023 \mathrm{mmol})$ in $\mathrm{CH}_{2} \mathrm{Cl}_{2}(2 \mathrm{~mL})$ was added pyridinium dichromate $(10 \mathrm{mg}, 0.027 \mathrm{mmol})$. The resulting mixture was stirred at room temperature for $4 \mathrm{~h}$ before it was quenched by the addition of $\mathrm{NaHCO}_{3}(5 \mathrm{~mL}$, satd aq). The layers were separated and the aqueous layer was extracted with $\mathrm{CH}_{2} \mathrm{Cl}_{2}(3 \times 10 \mathrm{~mL})$. The combined organic layers were washed with brine $(10 \mathrm{~mL})$, dried $\left(\mathrm{Na}_{2} \mathrm{SO}_{4}\right)$, and concentrated in vacuo. Flash column chromatography (silica gel, hexanes:EtOAc 1:1) afforded erinacerin A (2) (8 $\mathrm{mg}, 80 \%)$ as a colorless oil.

${ }^{1} \mathbf{H}$ NMR (500 MHz, $\left.\mathrm{CDCl}_{3}\right): \delta$ 7.31-7.28 (m, 2H, $\left.\mathrm{Ph} H_{m}\right), 7.25-7.21\left(\mathrm{~m}, 2 \mathrm{H}, \mathrm{Ph} H_{o}\right), 7.20(\mathrm{tt}, J=$ 7.1, $\left.1.4 \mathrm{~Hz}, 1 \mathrm{H}, \mathrm{Ph} H_{p}\right), 6.90$ (s, $\left.1 \mathrm{H}, \mathrm{Ar} H\right), 6.05$ (qq, $\left.J=1.2,1.2 \mathrm{~Hz}, 1 \mathrm{H}, \mathrm{C} 11 H\right), 4.16(\mathrm{~d}, J=17.0$ $\mathrm{Hz}, 1 \mathrm{H}, \mathrm{ArCH}_{a} \mathrm{HbN}_{b}$ ), 4.12 (d, $J=16.8 \mathrm{~Hz}, 1 \mathrm{H}, \mathrm{ArCH}_{\mathrm{a}} H_{b} \mathrm{~N}$ ), 3.87 (s, 3H, OCH3), 3.84 (nfot, 2H, $\mathrm{NCH}_{a} H_{a} \cdot \mathrm{CH}_{\mathrm{b}} \mathrm{H}_{\mathrm{b}} \mathrm{Ph}$ ), 2.98 (nfot, 2H, $\mathrm{NCH}_{a} \mathrm{H}_{\mathrm{a}} \mathrm{CH}_{b} H_{b}, \mathrm{Ph}$ ), 2.76 (d, $J=14.1 \mathrm{~Hz}, 1 \mathrm{H}, \mathrm{C} H_{a} \mathrm{H}_{\mathrm{b}}$ ), 2.73-2.67 (two overlapping nfom, $2 \mathrm{H}, \mathrm{C} 6 \mathrm{H}_{2}$ ), 2.64 (d, J=14.4 Hz, 1H, C9Ha $H_{b}$ ), 2.15 (d, 3H, J $\left.=1.2 \mathrm{~Hz}, \mathrm{C} 12 \mathrm{ECH}_{3}\right), 2.02\left(\mathrm{dt}, 1 \mathrm{H}, J=14,6.6 \mathrm{~Hz}, \mathrm{C} 7 \mathrm{H}_{a} \mathrm{Hb}_{\mathrm{b}}\right), 1.90\left(\mathrm{dt}, 1 \mathrm{H}, J=14,6.9 \mathrm{~Hz}, \mathrm{C} \mathrm{H}_{\mathrm{a}} H_{b}\right)$, $1.85\left(\mathrm{~d}, 3 \mathrm{H}, J=1.2 \mathrm{~Hz}, \mathrm{C} 12 \mathrm{ZCH}_{3}\right)$, and $1.41\left(\mathrm{~s}, 3 \mathrm{H}, \mathrm{C} 8 \mathrm{CH}_{3}\right)$.

${ }^{13} \mathrm{C}$ NMR (125 MHz, $\left.\mathrm{CDCl}_{3}\right): \delta 198.2,169.1,158.6,155.8,148.7,138.9,132.4,128.9,128.8$, 126.6, 125.2, 122.0, 113.7, 96.3, 76.1, 56.0, 52.4, 48.1, 44.3, 35.1, 30.8, 28.0, 24.9, 20.9, and 17.5.

HRMS (ESI) $m / z:[\mathrm{M}+\mathrm{H}]^{+}$Calcd for $\mathrm{C}_{27} \mathrm{H}_{32} \mathrm{NO}_{4}{ }^{+}$434.2326; Found 434.2307.

IR (neat): 2985, 2925, 2856, 1736, 1686, and $1607 \mathrm{~cm}^{-1}$. 
N-(2-((tert-Butyldiphenylsilyl)oxy)ethyl)prop-2-yn-1-amine (S9)

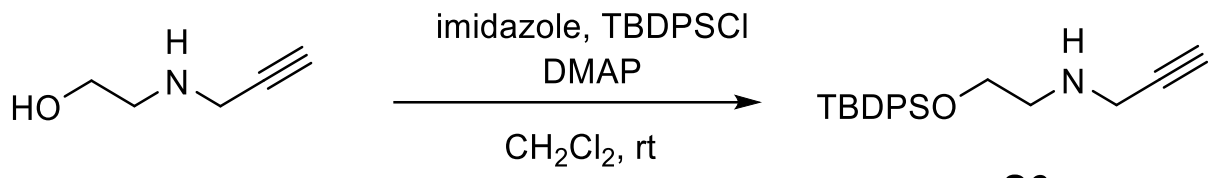

S9

To a stirred solution of 2-(prop-2-yn-1-ylamino)ethan-1-ol ${ }^{8}$ (1.58 g, $\left.15.9 \mathrm{mmol}\right)$ in THF (15 mL) was added imidazole $(1.20 \mathrm{~g}, 17.5 \mathrm{mmol})$, followed by tert-butylchlorodiphenylsilane $(4.81 \mathrm{~g}, 17.5$ mmol) and DMAP (194 mg, $1.59 \mathrm{mmol})$. The resulting mixture was stirred at room temperature for $2 \mathrm{~h}$ before it was quenched by the addition of $\mathrm{NH}_{4} \mathrm{Cl}(10 \mathrm{~mL}$, satd aq). The layers were separated and the aqueous layer was extracted with $\mathrm{CH}_{2} \mathrm{Cl}_{2}(3 \times 20 \mathrm{~mL})$. The combined organic layers were washed with brine $(20 \mathrm{~mL})$, dried $\left(\mathrm{Na}_{2} \mathrm{SO}_{4}\right)$, and concentrated in vacuo. Flash column chromatography (silica gel, hexanes:EtOAc 10:1) afforded $\mathbf{S 9}(4.74 \mathrm{~g}, 90 \%)$ as a colorless oil.

${ }^{1} \mathbf{H}$ NMR (400 MHz, $\left.\mathrm{CDCl}_{3}\right): \delta$ 7.69-7.65 (m, 4H, $\left.\mathrm{Ph} H_{m}\right), 7.44-7.35\left(\mathrm{~m}, 6 \mathrm{H}, \mathrm{Ph} H_{o}\right.$ and $\left.\mathrm{Ph} H_{p}\right)$, $3.79\left(\mathrm{t}, J=5.2 \mathrm{~Hz}, 2 \mathrm{H}, \mathrm{OCH}_{2} \mathrm{CH}_{2}\right), 3.45\left(\mathrm{~d}, J=2.4 \mathrm{~Hz}, 2 \mathrm{H}, \mathrm{CH}_{2} \mathrm{C} \equiv \mathrm{CH}\right), 2.84(\mathrm{t}, J=5.2 \mathrm{~Hz}, 2 \mathrm{H}$, $\left.\mathrm{OCH}_{2} \mathrm{CH}_{2}\right), 2.22\left(\mathrm{t}, J=2.4 \mathrm{~Hz}, 1 \mathrm{H}, \mathrm{CH}_{2} \mathrm{C} \equiv \mathrm{CH}\right)$, and $1.06\left[\mathrm{~s}, 9 \mathrm{H}, \mathrm{SiC}(\mathrm{CH})_{3}\right]$.

${ }^{13} \mathrm{C}$ NMR $\left(101 \mathrm{MHz}, \mathrm{CDCl}_{3}\right): \delta 135.7,133.7,130.3,127.8,82.4,71.9,65.8 .50 .1,36.5,27.0$, and 19.9 .

HRMS (ESI) $m / z:[\mathrm{M}+\mathrm{H}]^{+}$Calcd for $\mathrm{C}_{21} \mathrm{H}_{28} \mathrm{NOSi}^{+}$338.1935; Found 338.1921.

IR (neat): 3298, 3071, 3049, 2999, and $2890 \mathrm{~cm}^{-1}$. 


\section{$N$-(2-((tert-Butyldiphenylsilyl)oxy)ethyl)-3-(dimethylsilyl)prop-2-yn-1-amine (22)}

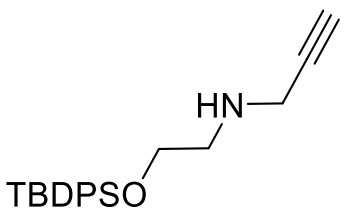

S9

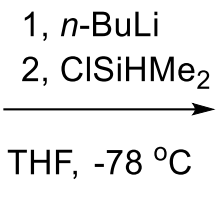

$\mathrm{THF},-78^{\circ} \mathrm{C}$

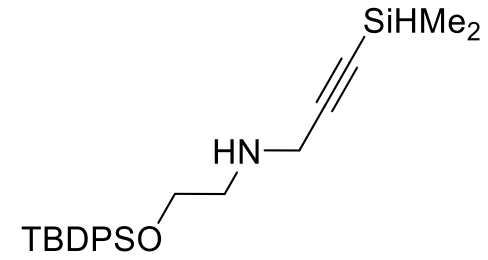

22

To a stirred solution of $\mathbf{S 9}(1.50 \mathrm{~g}, 4.44 \mathrm{mmol})$ in THF $(15 \mathrm{~mL})$ was added a solution of $n$-BuLi $(2.5 \mathrm{M}$ in hexanes, $1.78 \mathrm{~mL}, 4.44 \mathrm{mmol})$ dropwise at $-78^{\circ} \mathrm{C}$. The reaction mixture was stirred at $78{ }^{\circ} \mathrm{C}$ for $30 \mathrm{~min}$, followed by the addition of chlorodimethylsilane $(0.60 \mathrm{~mL}, 5.33 \mathrm{mmol})$ in THF $(2 \mathrm{~mL})$. The resulting mixture was allowed to warm to $0{ }^{\circ} \mathrm{C}$ and stirred for an additional $30 \mathrm{~min}$ before it was quenched by the addition of $\mathrm{NH}_{4} \mathrm{Cl}(15 \mathrm{~mL}$, satd aq). The layers were separated and the aqueous layer was extracted with $\mathrm{CH}_{2} \mathrm{Cl}_{2}(3 \times 20 \mathrm{~mL})$. The combined organic layers were washed with brine $(20 \mathrm{~mL})$, dried $\left(\mathrm{Na}_{2} \mathrm{SO}_{4}\right)$, and concentrated in vacuo. Flash column chromatography (silica gel, hexanes:EtOAc 4:1) afforded 22 (1.50 g, 87\%) as a light yellow oil.

${ }^{1} \mathbf{H}$ NMR (400 MHz, $\left.\mathrm{CDCl}_{3}\right): \delta$ 7.69-7.65 (m, 4H, $\left.\mathrm{Ph} H_{m}\right), 7.44-7.35\left(\mathrm{~m}, 6 \mathrm{H}, \mathrm{Ph} H_{o}\right.$ and $\left.\mathrm{Ph} H_{p}\right)$, 4.14 [sept, $J=3.7 \mathrm{~Hz}, 1 \mathrm{H}, \mathrm{Si} H\left(\mathrm{CH}_{3}\right)_{2}$ ], 3.79 (t, $\left.J=5.2 \mathrm{~Hz}, 2 \mathrm{H}, \mathrm{OCH}_{2} \mathrm{CH}_{2}\right), 3.48\left(\mathrm{~s}, 2 \mathrm{H}, \mathrm{CH}_{2} \mathrm{C} \equiv \mathrm{C}\right)$, $2.84\left(\mathrm{t}, J=5.2 \mathrm{~Hz}, 2 \mathrm{H}, \mathrm{OCH}_{2} \mathrm{CH}_{2}\right), 1.06\left[\mathrm{~s}, 9 \mathrm{H}, \mathrm{SiC}\left(\mathrm{CH}_{3}\right)_{3}\right]$, and $0.24[\mathrm{~d}, J=3.8 \mathrm{~Hz}, 6 \mathrm{H}$, $\left.\mathrm{SiH}\left(\mathrm{CH}_{3}\right)_{2}\right]$.

${ }^{13} \mathrm{C}$ NMR (101 MHz, $\left.\mathrm{CDCl}_{3}\right): \delta 135.7,133.7,129.8,127.8,106.2,85.0,63.3,50.6,39.4,27.0$, 19.4 , and -2.8 .

HRMS (ESI) $m / z:[\mathrm{M}+\mathrm{H}]^{+}$Calcd for $\mathrm{C}_{23} \mathrm{H}_{34} \mathrm{NOSi}_{2}+396.2173$; Found 396.2160.

IR (neat): 3302, 3070, 3049, 2999, 2956, 2890, 1825 (m), and $1589 \mathrm{~cm}^{-1}$. 


\section{8-Methylnona-7-en-2,4-diynal (S11)}
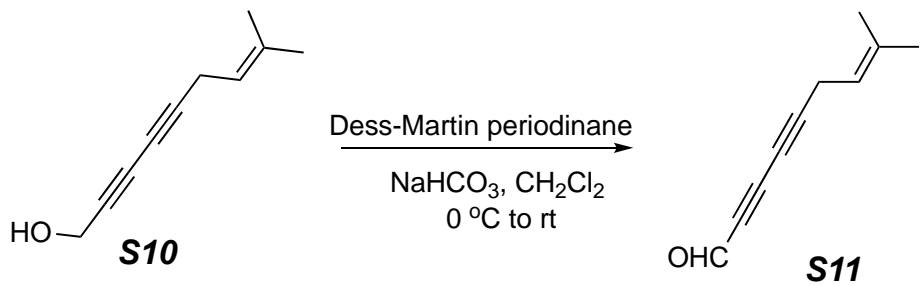

To a stirred solution of alcohol $\mathbf{S 1 0}^{9}$ (500 mg, $3.4 \mathrm{mmol}$ ) in $\mathrm{CH}_{2} \mathrm{Cl}_{2}\left(10 \mathrm{~mL}\right.$ ) were added $\mathrm{NaHCO}_{3}$ $(571 \mathrm{mg}, 6.8 \mathrm{mmol})$ and the Dess-Martin periodinane $(1.73 \mathrm{~g}, 4.08 \mathrm{mmol})$. The resulting mixture was stirred at room temperature for $30 \mathrm{~min}$ before it was quenched by the addition of $\mathrm{NaHCO}_{3}(5$ $\mathrm{mL}$, satd aq) and $\mathrm{Na}_{2} \mathrm{SO}_{3}(5 \mathrm{~mL}$, satd aq). The layers were separated and the aqueous layer was extracted with $\mathrm{CH}_{2} \mathrm{Cl}_{2}(3 \times 10 \mathrm{~mL})$. The combined organic layers were washed with brine $(20 \mathrm{~mL})$, dried $\left(\mathrm{Na}_{2} \mathrm{SO}_{4}\right)$, and concentrated in vacuo. Flash column chromatography (silica gel, hexanes:EtOAc 9:1) afforded $\mathbf{S 1 1}(420 \mathrm{mg}, 85 \%)$ as a colorless oil.

${ }^{1} \mathbf{H}$ NMR (400 MHz, $\mathrm{CDCl}_{3}$ ): $\delta 9.18(\mathrm{~s}, 1 \mathrm{H}, \mathrm{CHO}), 5.15$ (tqq, $\left.J=6.9,1.5,1.5 \mathrm{~Hz}, 1 \mathrm{H}, \mathrm{C} 7 \mathrm{H}\right), 3.08$ (dqq, $\left.J=6.9,1.1,1.1 \mathrm{~Hz}, 2 \mathrm{H}, \mathrm{C} \equiv \mathrm{CCH}_{2} \mathrm{CH}=\mathrm{C}\right), 1.73(\mathrm{dt}, J=1.4,1.4 \mathrm{~Hz}, 3 \mathrm{H}, \mathrm{C} 8 \mathrm{ECH})_{3}$ ), and 1.65 (dt, $J=1.3,0.9 \mathrm{~Hz}, 3 \mathrm{H}, \mathrm{C}_{2} \mathrm{ZCH} \mathrm{H}_{3}$ ).

${ }^{13} \mathrm{C}$ NMR (101 MHz, $\left.\mathrm{CDCl}_{3}\right): \delta 176.2,136.6,115.5,91.5,80.8,72.8,63.3,25.6,19.0$, and 18.0.

HRMS (CI) $m / z:\left[\mathrm{M}+\mathrm{NH}_{4}\right]^{+}$Calcd for $\mathrm{C}_{10} \mathrm{H}_{14} \mathrm{NO}^{+}$164.1070; Found 164.1068

IR (neat): 2972, 2915, 2858, 2736, 2227, 2130, and $1656 \mathrm{~cm}^{-1}$. 
8-Methylnona-7-en-2,4-diynoic acid (23)

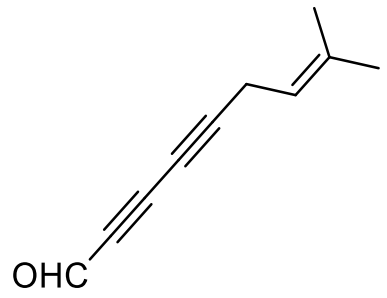

$S 11$

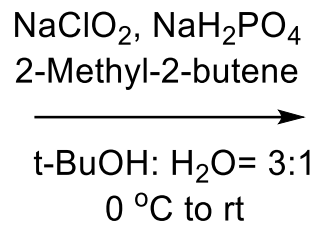

$0^{\circ} \mathrm{C}$ to rt

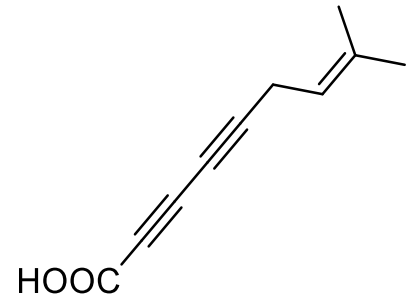

23

To a stirred solution of aldehyde $\mathbf{S 1 1}(500 \mathrm{mg}, 3.42 \mathrm{mmol})$ in $t-\mathrm{BuOH}(6 \mathrm{~mL})$ and $\mathrm{H}_{2} \mathrm{O}(2 \mathrm{~mL})$ were added $\mathrm{NaH}_{2} \mathrm{PO}_{4}(672 \mathrm{mg}, 6.85 \mathrm{mmol})$, 2-methyl-2-butene $(0.73 \mathrm{~mL}, 6.85 \mathrm{mmol})$, and $\mathrm{NaClO}_{2}(581 \mathrm{mg}, 5.14 \mathrm{mmol})$ at $0{ }^{\circ} \mathrm{C}$. The reaction mixture was then allowed to warm to room temperature. After being stirred for $1 \mathrm{~h}$ at room temperature, the reaction mixture was concentrated under reduced pressure, water was added, and the resulting mixture was extracted with ethyl acetate. The combined ethyl acetate layers were washed with brine, dried over anhydrous $\mathrm{Na}_{2} \mathrm{SO}_{4}$, filtered, and concentrated under reduced pressure to provide the crude acid $\mathbf{2 3}$, which was used for the next reaction without further purification.

${ }^{1} \mathbf{H}$ NMR (400 MHz, $\mathrm{CDCl}_{3}$ ): $\delta 10.43$ (br s, 1H, COOH), 5.16 (tqq, $J=6.9,1.5,1.5 \mathrm{~Hz}, 1 \mathrm{H}, \mathrm{C} 7 H$ ), 3.08 (dqq, $\left.J=6.9,1.1,1.1 \mathrm{~Hz}, 2 \mathrm{H}, \mathrm{C} \equiv \mathrm{CCH}_{2} \mathrm{CH}=\mathrm{C}\right), 1.75(\mathrm{dt}, J=1.4,1.4 \mathrm{~Hz}, 3 \mathrm{H}, \mathrm{C} 8 \mathrm{ECH})$, and $1.66\left(\mathrm{dt}, J=1.3,0.9 \mathrm{~Hz}, 3 \mathrm{H}, \mathrm{C}^{2} \mathrm{ZCH} \mathrm{CH}_{3}\right)$. 


\section{$N$-(2-((tert-Butyldiphenylsilyl)oxy)ethyl)- $N$-(3-(dimethylsilyl)prop-2-yn-1-yl)-8-methylnona-7-en-} 2,4-diynamide (24)

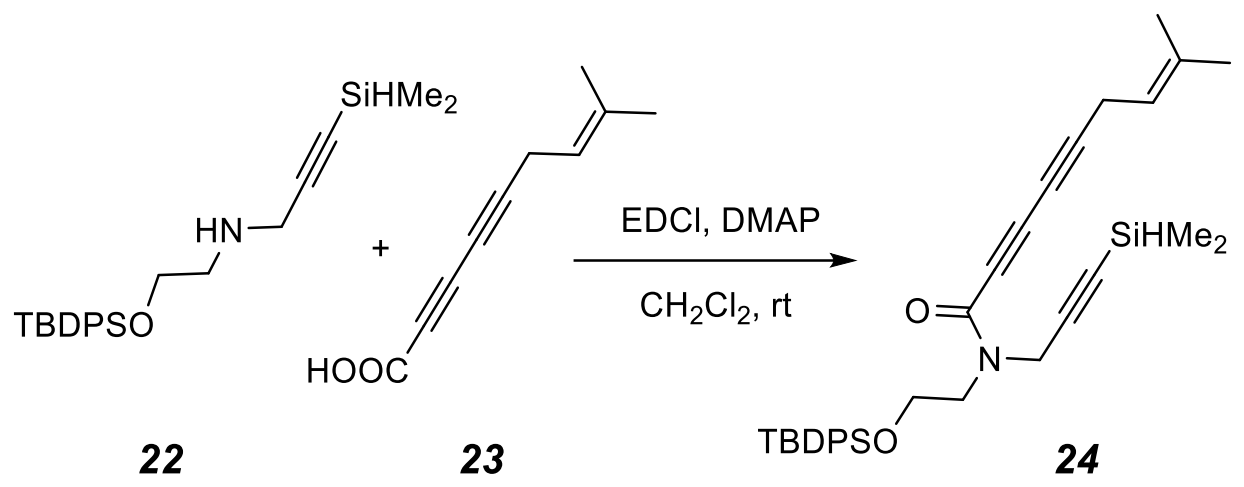

To a stirred solution of the amine $22(800 \mathrm{mg}, 2.02 \mathrm{mmol})$ in $\mathrm{CH}_{2} \mathrm{Cl}_{2}(10 \mathrm{~mL})$ at $0{ }^{\circ} \mathrm{C}$ was added EDCI (465 $\mathrm{mg}, 2.42 \mathrm{mmol}$ ) followed immediately by a solution of the acid $\mathbf{2 3}(394 \mathrm{mg}, 2.42 \mathrm{mmol})$ and DMAP (24.4 $\mathrm{mg}, 0.2 \mathrm{mmol})$ in $\mathrm{CH}_{2} \mathrm{Cl}_{2}(5 \mathrm{~mL})$ dropwise over ca. $5 \mathrm{~min}$. The resulting mixture was stirred at room temperature for $1 \mathrm{~h}$ before it was quenched by the addition of $\mathrm{NH}_{4} \mathrm{Cl}(10 \mathrm{~mL}$, satd aq). The layers were separated and the aqueous layer was extracted with $\mathrm{CH}_{2} \mathrm{Cl}_{2}(3 \times 20 \mathrm{~mL})$. The combined organic layers were washed with brine $(20 \mathrm{~mL})$, dried $\left(\mathrm{Na}_{2} \mathrm{SO}_{4}\right)$, and concentrated in vacuo. Flash column chromatography (silica gel, hexanes:EtOAc 10:1) afforded the amide 24 (764 mg, 70\%) as a light yellow oil.

Two rotamers for the tertiary amide (ca. 2:1) were observed in the NMR spectral data:

${ }^{1} \mathbf{H}$ NMR $\left(400 \mathrm{MHz}, \mathrm{CDCl}_{3}\right): \delta$ 7.67-7.60 (m, 4H, $\left.\mathrm{Ph} H\right), 7.49-7.36(\mathrm{~m}, 6 \mathrm{H}, \mathrm{Ph} H), 5.19-5.10(\mathrm{~m}, 1 \mathrm{H}$, $\left.\mathrm{C}=\mathrm{CHCH}_{2}\right), 4.50$ (s, ca. $0.65 \mathrm{H}, \mathrm{C} \equiv \mathrm{CCH}_{2} \mathrm{~N}$, minor), 4.29 (s, ca.1.35H, $\mathrm{C} \equiv \mathrm{CCH}_{2} \mathrm{~N}$, major), 4.13 [sept, $J=$ $3.7 \mathrm{~Hz}$, ca. $0.33 \mathrm{H}, \mathrm{Si} H\left(\mathrm{CH}_{3}\right)_{2}$, minor], 4.08 [sept, $J=3.8 \mathrm{~Hz}$, ca. $0.67 \mathrm{H}, \mathrm{Si} H\left(\mathrm{CH}_{3}\right)_{2}$, major], 3.89-3.75 (m, $4 \mathrm{H}, \mathrm{OCH}_{2} \mathrm{CH}_{2}$ ), 3.05 (br d, $J=7.0 \mathrm{~Hz}, 0.65 \mathrm{H}, \mathrm{C} \equiv \mathrm{CCH}_{2} \mathrm{CH}=\mathrm{C}$, minor), 3.03 (br d, $J=6.9 \mathrm{~Hz}$, ca. $1.35 \mathrm{H}$, $\mathrm{C} \equiv \mathrm{CCH}_{2} \mathrm{CH}=\mathrm{C}$, major), $1.74\left(\mathrm{~d}, J=1.4 \mathrm{~Hz}, 1 \mathrm{H}, \mathrm{C} 8 \mathrm{ECH}_{3}\right.$, minor), $1.72\left(\mathrm{~d}, J=1.4 \mathrm{~Hz}, 2.0 \mathrm{H}, \mathrm{C} 8 \mathrm{ECH} H_{3}\right.$, major), $1.66\left(\mathrm{~d}, J=1.3 \mathrm{~Hz}, 1 \mathrm{H}, \mathrm{C} 8 \mathrm{ZCH}_{3}\right.$, minor), $1.63\left(\mathrm{~d}, J=1.3 \mathrm{~Hz}, 2 \mathrm{H}, \mathrm{C} 8 \mathrm{ZCH}_{3}\right), 1.06$ [s, 9H, $\left.\mathrm{SiC}\left(\mathrm{CH}_{3}\right)_{3}\right]$, 0.24 [d, $J=3.8 \mathrm{~Hz}$, ca. $2 \mathrm{H}, \mathrm{SiH}\left(\mathrm{CH}_{3}\right)_{2}$, minor], and 0.20 [d, $J=3.8 \mathrm{~Hz}$, ca. $4 \mathrm{H}, \mathrm{SiH}\left(\mathrm{CH}_{3}\right)_{2}$, major].

${ }^{13}$ C NMR (101 MHz, $\left.\mathrm{CDCl}_{3}\right): \delta$ 153.6, 153.3, 136.2, 136.1, 135.71, 135.64, 133.3, 133.1, 129.98, 129.97, 128.0, 127.9, 116.12, 116.07, 101.12, 101.10, 87.4, 86.7, 86.6, 86.4, 76.6, 76.4, 66.6, 66.3, 63.6, 63.5, 62.2, $61.9,49.7,46.2,41.1,35.0,27.0,26.9,25.63,25.61,19.24,19.22,18.81,18.78,17.98,17.96$, and $-3.05(x 2)$.

HRMS (ESI) $m / z:[\mathrm{M}+\mathrm{H}]^{+}$Calcd for $\mathrm{C}_{33} \mathrm{H}_{42} \mathrm{NO}_{2} \mathrm{Si}_{2}{ }^{+}$540.2749; Found 540.2741.

IR (neat): 3254, 3071, 3049, 2960, 2320 (s), 2177 (m), 1734, and $1589 \mathrm{~cm}^{-1}$. 
2-(2-((tert-Butyldiphenylsilyl)oxy)ethyl)-7-(dimethylsilyl)-6-(3-methylbut-2-en-1-yl)-3oxoisoindolin-5-yl acetate (26a)

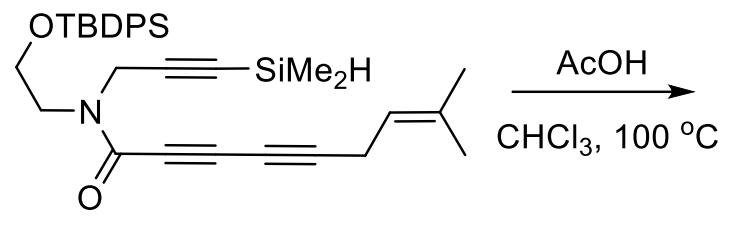

24<smiles>[B-]C([18F])=CCc1c(OC(C)=O)cc2c(c1SC)CN(CC[OH+])C2=O</smiles>

$26 a$

A solution of $24(500 \mathrm{mg}, 0.93 \mathrm{mmol})$ and acetic acid $(0.55 \mathrm{~mL}, 9.3 \mathrm{mmol})$ in chloroform $(10 \mathrm{~mL})$ was heated in a screw-capped culture tube in an oil bath held at $100{ }^{\circ} \mathrm{C}$. After $4 \mathrm{~h}$, the resulting solution was allowed to cool to room temperature and concentrated in vacuo. Flash column chromatography (silica gel, hexanes:EtOAc 5:1) of the residue afforded 26a (472 $\mathrm{mg}, 85 \%)$ as a colorless oil.

${ }^{1} \mathbf{H}$ NMR (400 MHz, $\mathrm{CDCl}_{3}$ ): $\delta$ 7.64-7.28 (m, $11 \mathrm{H}, \mathrm{Ph} H$ and $\mathrm{Ar} H$ ), 4.96 (tqq, $J=4.6,1.4,1.4 \mathrm{~Hz}$, $\left.1 \mathrm{H}, \mathrm{C}=\mathrm{CHCH}_{2}\right), 4.62\left(\mathrm{~s}, 2 \mathrm{H}, \mathrm{ArCH}_{2} \mathrm{~N}\right), 4.55$ [sept, $\left.J=4.0 \mathrm{~Hz}, 1 \mathrm{H}, \mathrm{Si} H\left(\mathrm{CH}_{3}\right)_{2}\right], 3.91(\operatorname{app~t}, J=$ $4.9 \mathrm{~Hz}, 2 \mathrm{H}, \mathrm{OCH}_{2} \mathrm{CH}_{2}$ ), 3.74 (app t, $J=5.0 \mathrm{~Hz}, 2 \mathrm{H}, \mathrm{OCH}_{2} \mathrm{CH}_{2}$ ), 3.46 (dqq, $J=6.1,1.3,1.3 \mathrm{~Hz}$, $\left.2 \mathrm{H}, \mathrm{ArCH}_{2} \mathrm{CH}=\mathrm{C}\right), 2.28\left(\mathrm{~s}, 3 \mathrm{H}, \mathrm{COCH}_{3}\right), 1.75\left(\mathrm{dt}, J=1.2,1.2 \mathrm{~Hz}, 3 \mathrm{H}, \mathrm{C} 5{ }^{\prime} \mathrm{H}_{3}\right), 1.70(\mathrm{dt}, J=1.6$, $\left.1.6 \mathrm{~Hz}, 3 \mathrm{H}, \mathrm{C} 4{ }^{\prime \prime} \mathrm{CH}_{3}\right), 1.06\left[\mathrm{~s}, 9 \mathrm{H}, \mathrm{SiC}\left(\mathrm{CH}_{3}\right)_{3}\right]$, and 0.37 [d, $\left.J=4.0 \mathrm{~Hz}, 6 \mathrm{H}, \mathrm{SiH}\left(\mathrm{CH}_{3}\right)_{2}\right]$.

${ }^{13} \mathrm{C}$ NMR $\left(101 \mathrm{MHz}, \mathrm{CDCl}_{3}\right): \delta 169.6,167.9,149.5,145.7,143.2,135.6,133.2,133.1,132.4$, $131.7,130.0,127.9,122.6,119.3,63.7,54.0,45.1,30.6,27.0,25.7,21.0,19.3,18.4$, and -2.8 .

HRMS (ESI) $m / z:[\mathrm{M}+\mathrm{H}]^{+}$Calcd for $\mathrm{C}_{35} \mathrm{H}_{46} \mathrm{NO}_{4} \mathrm{Si}_{2}{ }^{+}$600.2960; Found 600.2950.

IR (neat): 3073, 2956, 2866, $2136(\mathrm{~m}), 1768$, and $1685 \mathrm{~cm}^{-1}$. 
2-(2-((tert-Butyldiphenylsilyl)oxy)ethyl)-4-(dimethylsilyl)-6-hydroxy-5-(3-methylbut- 2-en1-yl)isoindolin-1-one (26b)

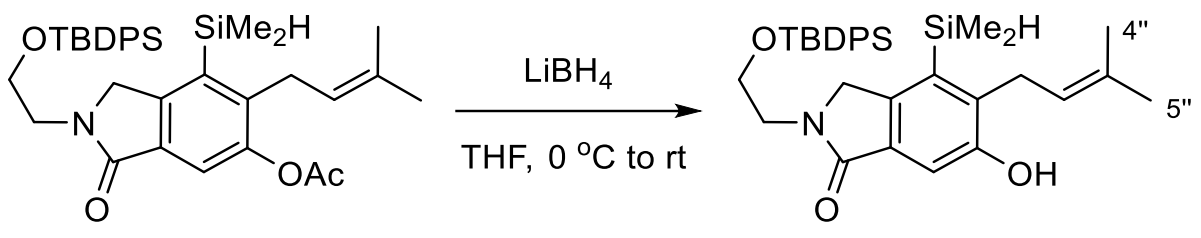

$26 a$

$26 b$

To a stirred solution of the amine $26 \mathbf{a}(200 \mathrm{mg}, 0.33 \mathrm{mmol})$ in THF $(10 \mathrm{~mL})$ at $0{ }^{\circ} \mathrm{C}$ was added $\mathrm{LiBH}_{4}(2.5 \mathrm{M}$ in THF, $0.2 \mathrm{~mL}, 0.4 \mathrm{mmol})$. The resulting mixture was stirred at room temperature for $1 \mathrm{~h}$ before it was quenched by the addition of $\mathrm{NH}_{4} \mathrm{Cl}(10 \mathrm{~mL}$, satd aq). The layers were separated and the aqueous layer was extracted with $\mathrm{CH}_{2} \mathrm{Cl}_{2}(3 \times 20 \mathrm{~mL})$. The combined organic layers were washed with brine $(20 \mathrm{~mL})$, dried $\left(\mathrm{Na}_{2} \mathrm{SO}_{4}\right)$, and concentrated in vacuo. Flash column chromatography (silica gel, hexanes:EtOAc 10:1) of the residue afforded the phenolic lactam 26b (167 mg, 90\%) as a colorless oil.

${ }^{1} \mathbf{H}$ NMR (400 MHz, $\left.\mathrm{CDCl}_{3}\right): \delta 7.67(\mathrm{~m}, 1 \mathrm{H}, \mathrm{Ar} H), 7.60\left(\operatorname{app~d}, J=7 \mathrm{~Hz}, 4 \mathrm{H}, \mathrm{Ph} H_{o}\right), 7.38(\mathrm{tt}, J=$ 7.3, $1.4 \mathrm{~Hz}, 2 \mathrm{H}, \mathrm{Ph} H_{p}$ ), 7.30 (app t, $J=7 \mathrm{~Hz}, 4 \mathrm{H}, \mathrm{Ph} H_{m}$ ), 7.12 (br s, 1H, OH), 5.12 (tqq, $J=6.2$, 1.4, $\left.1.4 \mathrm{~Hz}, 1 \mathrm{H}, \mathrm{C}=\mathrm{CHCH}_{2}\right), 4.57$ (s, $\left.2 \mathrm{H}, \mathrm{ArCH}_{2} \mathrm{~N}\right), 4.54$ [sept, $J=4.0 \mathrm{~Hz}, 1 \mathrm{H}, \mathrm{Si} H\left(\mathrm{CH}_{3}\right)_{2}$ ], 3.92 $\left(\operatorname{app~t}, J=5.1 \mathrm{~Hz}, 2 \mathrm{H}, \mathrm{OCH}_{2} \mathrm{CH}_{2}\right), 3.76\left(\operatorname{app~t}, J=5.0 \mathrm{~Hz}, 2 \mathrm{H}, \mathrm{OCH}_{2} \mathrm{CH}_{2}\right), 3.55$ (dqq, $J=6.2,1.3$, $\left.1.3 \mathrm{~Hz}, 2 \mathrm{H}, \mathrm{ArCH}_{2} \mathrm{CH}=\mathrm{C}\right), 1.79\left(\mathrm{dt}, J=0.9,1.2 \mathrm{~Hz}, 3 \mathrm{H}, \mathrm{C} 5{ }^{\prime} \mathrm{H}_{3}\right), 1.72(\mathrm{dt}, J=1.5,1.5 \mathrm{~Hz}, 3 \mathrm{H}$, $\left.\mathrm{C} 4{ }^{\prime} \mathrm{H}_{3}\right), 1.05\left[\mathrm{~s}, 9 \mathrm{H}, \mathrm{SiC}\left(\mathrm{CH}_{3}\right)_{3}\right]$, and $0.37\left[\mathrm{~d}, J=4.0 \mathrm{~Hz}, 6 \mathrm{H}, \mathrm{SiH}\left(\mathrm{CH}_{3}\right)_{2}\right]$.

${ }^{13} \mathrm{C}$ NMR (101 MHz, $\left.\mathrm{CDCl}_{3}\right): \delta 169.2,155.3,139.7,138.3,135.6,133.6,133.3,131.7,131.3$, $129.9,127.9,122.7,112.0,63.5,54.0,45.2,30.3,27.0,25.9,19.3,18.4$, and -2.7 .

HRMS (ESI) $m / z:[\mathrm{M}+\mathrm{H}]^{+}$Calcd for $\mathrm{C}_{33} \mathrm{H}_{44} \mathrm{NO}_{3} \mathrm{Si}_{2}{ }^{+}$558.2854; Found 558.2836.

IR (neat): 3234, 3073, 3045, 2960, 2930, 2863, 2128 (m), 1664, 1604, and $1582 \mathrm{~cm}^{-1}$. 
2-(2-((tert-Butyldiphenylsilyl)oxy)ethyl)-7-(dimethylsilyl)-6-(3-methylbut-2-en-1-yl)-3oxoisoindolin-5-yl 2-Hydroxy-4-(methoxymethoxy)-6-methylbenzoate (27a)

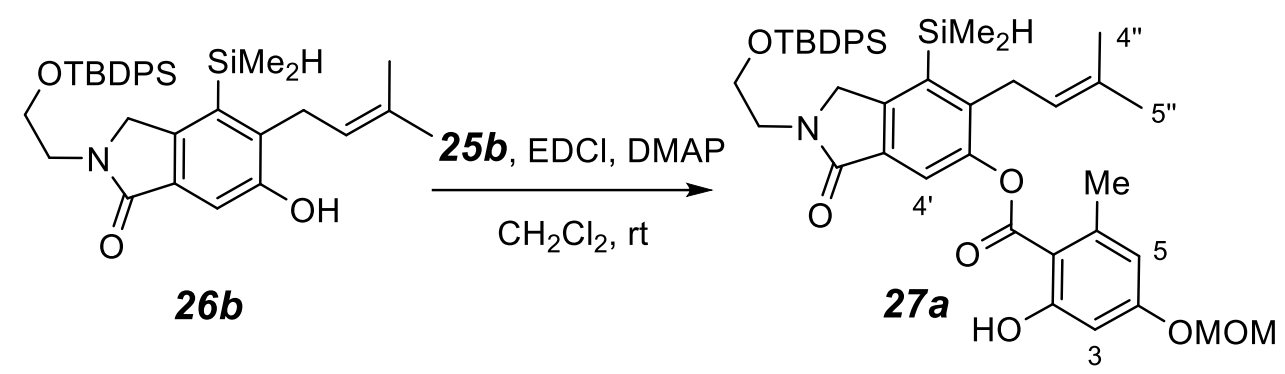

To a stirred solution of the phenol $26 \mathbf{b}(150 \mathrm{mg}, 0.27 \mathrm{mmol})$ in $\mathrm{CH}_{2} \mathrm{Cl}_{2}(10 \mathrm{~mL})$ at $0{ }^{\circ} \mathrm{C}$ was added EDCI (61.8 mg, $0.32 \mathrm{mmol})$ followed immediately by a solution of the acid $\mathbf{2 5} \mathbf{b}^{10}$ (68.4 $\mathrm{mg}, 0.32$ mmol) and DMAP (3 mg, $0.03 \mathrm{mmol})$ in $\mathrm{CH}_{2} \mathrm{Cl}_{2}(5 \mathrm{~mL})$ dropwise over ca. $5 \mathrm{~min}$. The resulting mixture was stirred at room temperature for $1 \mathrm{~h}$ before it was quenched by the addition of $\mathrm{NH}_{4} \mathrm{Cl}$ (10 mL, satd aq). The layers were separated and the aqueous layer was extracted with $\mathrm{CH}_{2} \mathrm{Cl}_{2}$ $(3 \times 20 \mathrm{~mL})$. The combined organic layers were washed with brine $(20 \mathrm{~mL})$, dried $\left(\mathrm{Na}_{2} \mathrm{SO}_{4}\right)$, and concentrated in vacuo. Flash column chromatography (silica gel, hexanes:EtOAc 10:1) afforded the ester $27 \mathbf{a}(150 \mathrm{mg}, 75 \%)$ as a light yellow oil.

${ }^{1}$ H NMR (400 MHz, $\left.\mathrm{CDCl}_{3}\right): \delta 11.36(\mathrm{~s}, 1 \mathrm{H}, \mathrm{ArOH}), 7.61$ (app d, $\left.J=7 \mathrm{~Hz}, 4 \mathrm{H}, \mathrm{Ph} H_{o}\right), 7.57$ (s, 1H, ArH4'), 7.41 (tt, $J=7.4,1.3 \mathrm{~Hz}, 2 \mathrm{H}, \mathrm{Ph} H_{p}$ ), 7.33 (app t, $J=7 \mathrm{~Hz}, 4 \mathrm{H}, \mathrm{Ph} H_{m}$ ), 6.54 (d, $J=2.6$ $\mathrm{Hz}, 1 \mathrm{H}, \mathrm{ArH3}$ ), 6.47 (br d, $J=2.5 \mathrm{~Hz}, 1 \mathrm{H}, \mathrm{ArH5}$ ), 5.22 (s, 2H, OCH $2 \mathrm{O}$ ), 4.95 (tqq, J = 6.0, 1.4, $\left.1.4 \mathrm{~Hz}, 1 \mathrm{H}, \mathrm{C}=\mathrm{CHCH}_{2}\right), 4.67\left(\mathrm{~s}, 2 \mathrm{H}, \mathrm{ArCH}_{2} \mathrm{~N}\right), 4.58$ [sept, $J=4.0 \mathrm{~Hz}, 1 \mathrm{H}, \mathrm{SiH}\left(\mathrm{CH}_{3}\right)_{2}$ ], 3.92 (app $\left.\mathrm{t}, J=4.9 \mathrm{~Hz}, 2 \mathrm{H}, \mathrm{OCH}_{2} \mathrm{CH}_{2}\right), 3.76\left(\operatorname{app~t}, J=5.0 \mathrm{~Hz}, 2 \mathrm{H}, \mathrm{OCH}_{2} \mathrm{CH}_{2}\right), 3.50\left(\mathrm{~s}, 3 \mathrm{H}, \mathrm{OCH}_{3}\right), 3.47$ (nfom, 2H, $\mathrm{ArCH}_{2} \mathrm{CH}=\mathrm{C}$ ), 2.65 (br s, 3H, $\mathrm{ArCH}_{3}$ ), 1.57 (br d, $J=1.4 \mathrm{~Hz}, 3 \mathrm{H}, \mathrm{C} 5$ ' 'H $\mathrm{H}_{3}$ ), 1.44 (br d, $\left.J=1.4 \mathrm{~Hz}, 3 \mathrm{H}, \mathrm{C} 4{ }^{\prime} \mathrm{H}_{3}\right), 1.07$ [s, 9H, $\left.\mathrm{SiC}\left(\mathrm{CH}_{3}\right)_{3}\right]$, and 0.39 [d, $\left.J=4.0 \mathrm{~Hz}, 6 \mathrm{H}, \mathrm{SiH}\left(\mathrm{CH}_{3}\right)_{2}\right]$.

${ }^{13} \mathrm{C}$ NMR (101 MHz, $\left.\mathrm{CDCl}_{3}\right): \delta 170.7,167.7,166.1,162.3,149.1,146.1,143.9,143.4,135.6$, 133.6, 133.2, 133.1, 132.0, 130.0, 127.9, 122.1, 119.3, 112.4, 105.4, 101.7, 94.1, 63.7, 56.5, 54.0, $45.2,30.8,27.0,25.5,24.7,19.3,18.0$, and -2.8 .

HRMS (ESI) $m / z:[\mathrm{M}+\mathrm{H}]^{+}$Calcd for $\mathrm{C}_{43} \mathrm{H}_{54} \mathrm{NO}_{7} \mathrm{Si}_{2}{ }^{+}$752.3433; Found 752.3419.

IR (neat): 3379, 3345, 3302, 3069, 2988, 2886, 2859, 2139 (m), 1689, 1659, 1615, and $1576 \mathrm{~cm}^{-}$ 1 . 
2-(2-((tert-Butyldiphenylsilyl)oxy)ethyl)-7-(dimethylsilyl)-6-(3-methylbut-2-en-1-yl)-3oxoisoindolin-5-yl 2-Hydroxy-4-(methoxymethoxy)-6-methylbenzoate (27a)

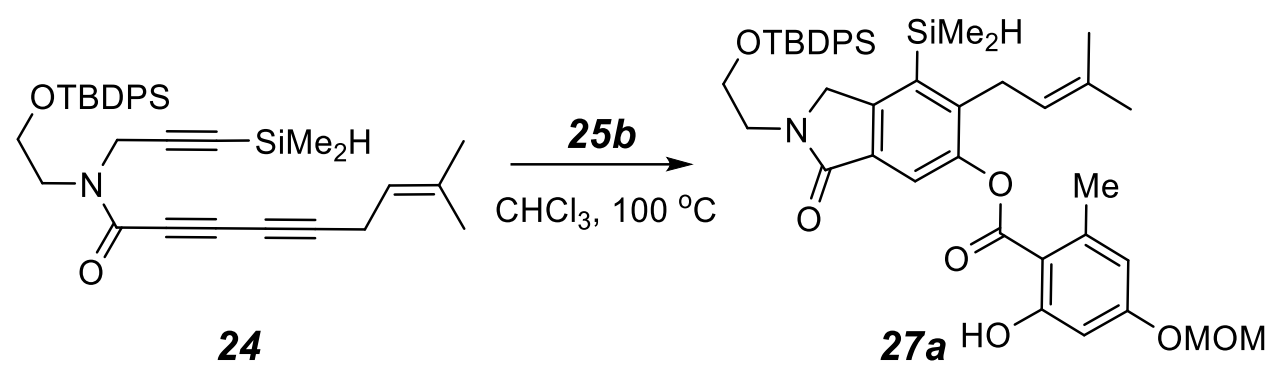

A solution of the triyne $24(95 \mathrm{mg}, 0.18 \mathrm{mmol})$ and acid $\mathbf{2 5 b}(76 \mathrm{mg}, 0.36 \mathrm{mmol})$ in chloroform $(5 \mathrm{~mL})$ was heated in a screw-capped culture tube in an oil bath held at $100{ }^{\circ} \mathrm{C}$. After $4 \mathrm{~h}$, the slightly yellow-colored solution was allowed to cool to room temperature and concentrated in vacuo. Flash column chromatography (silica gel, hexanes:EtOAc 5:1) afforded 27a (35 mg, 30\%) as a light yellow oil. This sample had essentially the same tlc and ${ }^{1} \mathrm{H}$ NMR spectral data as the sample prepared from esterification of the phenol. 
2-(2-((tert-Butyldiphenylsilyl)oxy)ethyl)-7-(hydroxydimethylsilyl)-6-(3-methylbut-2-en-1yl)-3-oxoisoindolin-5-yl 2-Hydroxy-4-(methoxymethoxy)-6-methylbenzoate (S12)

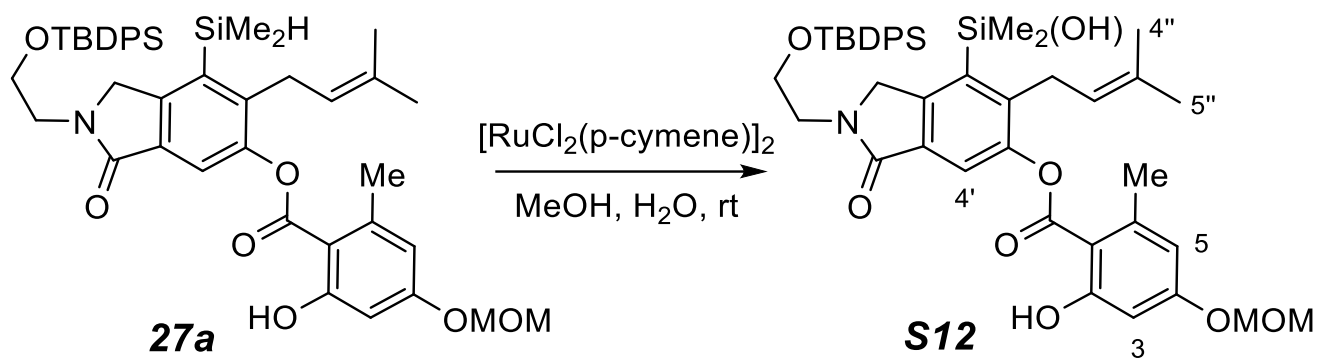

To a stirred solution of 27a $(80 \mathrm{mg}, 0.11 \mathrm{mmol})$ in $\mathrm{MeOH}(3 \mathrm{~mL})$ and $\mathrm{H}_{2} \mathrm{O}(3 \mathrm{~mL})$ was added $\left[\mathrm{RuCl}_{2}(p \text {-cymene })\right]_{2}(4.0 \mathrm{mg}, 0.006 \mathrm{mmol})$. The resulting mixture was stirred at room temperature for 30 min before it was quenched by the addition of $\mathrm{NaHCO}_{3}(10 \mathrm{~mL}$, satd aq). The layers were separated and the aqueous layer was extracted with $\mathrm{CH}_{2} \mathrm{Cl}_{2}(3 \times 10 \mathrm{~mL})$. The combined organic layers were washed with brine $(15 \mathrm{~mL})$, dried $\left(\mathrm{Na}_{2} \mathrm{SO}_{4}\right)$, and concentrated in vacuo. Flash column chromatography (silica gel, hexanes:EtOAc 3:1) of the residue afforded the silanol S12 (65 mg, $80 \%$ ) as a colorless oil.

${ }^{1} \mathbf{H}$ NMR (400 MHz, $\left.\mathrm{CDCl}_{3}\right): \delta\left(400 \mathrm{MHz}, \mathrm{CDCl}_{3}\right): \delta 11.37$ (s, 1H, $\left.\mathrm{ArOH}\right), 7.60$ (app d, $J=7 \mathrm{~Hz}$, 4H, $\mathrm{Ph} H_{o}$ ), 7.57 (s, 1H, ArH4'), 7.40 (tt, $J=7.4,1.4 \mathrm{~Hz}, 2 \mathrm{H}, \mathrm{Ph} H_{p}$ ), 7.33 (app t, $J=7 \mathrm{~Hz}, 4 \mathrm{H}$, $\left.\mathrm{Ph} H_{m}\right), 6.54(\mathrm{~d}, J=2.5 \mathrm{~Hz}, 1 \mathrm{H}, \mathrm{ArH3}), 6.47$ (br d, $\left.J=2.5 \mathrm{~Hz}, 1 \mathrm{H}, \mathrm{ArH5}\right), 5.22\left(\mathrm{~s}, 2 \mathrm{H}, \mathrm{OCH}_{2} \mathrm{O}\right)$, 4.95 (tqq, $\left.J=6.0,1.4,1.4 \mathrm{~Hz}, 1 \mathrm{H}, \mathrm{C}=\mathrm{CHCH}_{2}\right), 4.73\left(\mathrm{~s}, 2 \mathrm{H}, \mathrm{ArCH}_{2} \mathrm{~N}\right), 3.93(\operatorname{app~t}, J=5.0 \mathrm{~Hz}, 2 \mathrm{H}$, $\mathrm{OCH}_{2} \mathrm{CH}_{2}$ ), 3.73 (app t, $\left.J=5.0 \mathrm{~Hz}, 2 \mathrm{H}, \mathrm{OCH}_{2} \mathrm{CH}_{2}\right), 3.50\left(\mathrm{~s}, 3 \mathrm{H}, \mathrm{OCH}_{3}\right), 3.48-3.44(\mathrm{~m}, 2 \mathrm{H}$, $\mathrm{ArCH}_{2} \mathrm{CH}=\mathrm{C}$ ), 2.64 (s, 3H, $\left.\mathrm{ArCH}_{3}\right), 1.56$ (dt, $\left.J=1.6,1.6 \mathrm{~Hz}, 3 \mathrm{H}, \mathrm{C} 5{ }^{\prime}{ }^{\prime} \mathrm{H}_{3}\right), 1.42$ (br d, $J=1.3 \mathrm{~Hz}$, $\left.3 \mathrm{H}, \mathrm{C} 4{ }^{\prime} \mathrm{H}_{3}\right), 1.06$ [s, 9H, $\left.\mathrm{SiC}\left(\mathrm{CH}_{3}\right)_{3}\right]$, and $0.45\left[\mathrm{~d}, \mathrm{~J}=4.0 \mathrm{~Hz}, 6 \mathrm{H}, \mathrm{HOSi}\left(\mathrm{CH}_{3}\right)_{2}\right]$.

${ }^{13} \mathrm{C}$ NMR (101 MHz, $\left.\mathrm{CDCl}_{3}\right): \delta 170.7,167.5,166.1,162.3,149.2,145.7,143.9,142.4,135.6$, $135.2,133.5,133.4,132.3,130.0,127.90,122.3,119.4,112.4,105.7,101.7,94.1,63.5,56.5,54.6$, $45.1,30.7,27.0,25.4,24.7,19.4,18.0$, and 3.1.

HRMS (ESI) $m / z:[\mathrm{M}+\mathrm{H}]^{+}$Calcd for $\mathrm{C}_{43} \mathrm{H}_{54} \mathrm{NO}_{8} \mathrm{Si}_{2}{ }^{+}$768.3382; Found 768.3363.

IR (neat): 3070 (br), 3052, 2957, 1761, 1660, 1608, and $1554 \mathrm{~cm}^{-1}$. 
2-(2-((tert-Butyldiphenylsilyl)oxy)ethyl)-7-hydroxy-6-(3-methylbut-2-en-1-yl)-3-oxoisoindolin-5-yl 2-Hydroxy-4-(methoxymethoxy)-6-methylbenzoate (29)

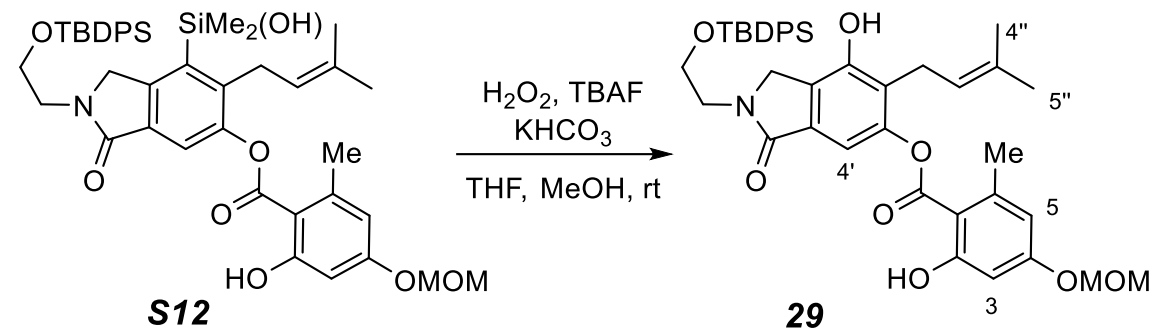

To a stirred solution of $\mathbf{S 1 2}(20 \mathrm{mg}, 0.03 \mathrm{mmol})$ in $\mathrm{MeOH}(2 \mathrm{~mL})$ and THF (2 $\mathrm{mL})$ was added $\mathrm{KHCO}_{3}(3 \mathrm{mg}, 0.06 \mathrm{mmol}), \mathrm{H}_{2} \mathrm{O}_{2}\left(30 \mathrm{wt} . \%\right.$ in $\left.\mathrm{H}_{2} \mathrm{O}, 36 \mu \mathrm{L}, 0.3 \mathrm{mmol}\right)$, and TBAF (1.0 M in THF, $6 \mu \mathrm{L}, 0.006 \mathrm{mmol})$. The resulting mixture was stirred at room temperature for $2 \mathrm{~h}$ before it was quenched by the addition of $\mathrm{NaHCO}_{3}(10 \mathrm{~mL}$, satd aq). The layers were separated and the aqueous layer was extracted with $\mathrm{CH}_{2} \mathrm{Cl}_{2}(3 \times 10 \mathrm{~mL})$. The combined organic layers were washed with brine $(20 \mathrm{~mL})$, dried $\left(\mathrm{Na}_{2} \mathrm{SO}_{4}\right)$, and concentrated in vacuo. Flash column chromatography (silica gel, hexanes:EtOAc 4:1) of the residue afforded the TBDPS-MOM ether (29) (12 mg, 65\%) as a colorless oil.

The NMR spectral data for this material compared favorably with those reported earlier for this substance. ${ }^{10}$

${ }^{1} \mathbf{H}$ NMR (500 MHz, CDCl $): \delta 11.34(\mathrm{~s}, 1 \mathrm{H}, \mathrm{ArOH}), 7.61\left(\operatorname{app~d}, J=7 \mathrm{~Hz}, 4 \mathrm{H}, \mathrm{Ph} H_{o}\right), 7.40$ (tt, $J$ $\left.=7.4,1.2 \mathrm{~Hz}, 2 \mathrm{H}, \mathrm{Ph} H_{p}\right), 7.33\left(\operatorname{app~t}, J=7 \mathrm{~Hz}, 4 \mathrm{H}, \mathrm{Ph} H_{m}\right), 7.21$ (s, 1H,ArH4'), 6.55 (d, J = 2.6 $\mathrm{Hz}, 1 \mathrm{H}, \mathrm{ArH3}$ ), 6.49 (br d, $J=2.5 \mathrm{~Hz}, 1 \mathrm{H}, \mathrm{ArH5}$ ), 5.24 (tqq, $J=7.0,1.3,1.3 \mathrm{~Hz}, 1 \mathrm{H}, \mathrm{C}=\mathrm{CHCH}_{2}$ ), $5.21\left(\mathrm{~s}, 2 \mathrm{H}, \mathrm{OCH}_{2} \mathrm{O}\right), 4.45\left(\mathrm{~s}, 2 \mathrm{H}, \mathrm{ArCH}_{2} \mathrm{~N}\right), 3.90\left(\mathrm{t}, J=5.1 \mathrm{~Hz}, 2 \mathrm{H}, \mathrm{OCH}_{2} \mathrm{CH}_{2}\right), 3.73(\mathrm{t}, J=5.1$ $\left.\mathrm{Hz}, 2 \mathrm{H}, \mathrm{OCH}_{2} \mathrm{CH}_{2}\right), 3.49$ (s, 3H, $\left.\mathrm{OCH}_{3}\right), 3.36$ (br d, $\left.J=7.0 \mathrm{~Hz}, 2 \mathrm{H}, \mathrm{ArCH}_{2} \mathrm{CH}=\mathrm{C}\right), 2.68(\mathrm{~s}, 3 \mathrm{H}$, $\left.\mathrm{ArCH}_{3}\right), 1.76\left(\mathrm{~s}, 6 \mathrm{H}, \mathrm{C} 5{ }^{\prime \prime} \mathrm{H}_{3}\right.$ and $\left.\mathrm{C} 4{ }^{\prime}{ }^{\prime} H_{3}\right)$, and 1.05 [s, 9H, $\left.\mathrm{SiC}\left(\mathrm{CH}_{3}\right)_{3}\right]$.

${ }^{13} \mathrm{C}$ NMR $\left(125 \mathrm{MHz}, \mathrm{CDCl}_{3}\right): \delta$ 170.4, 167.8, 166.1, 162.4, 150.8, 148.8, 143.7, 137.3, 135.5, 133.1, 132.8, 129.7, 127.7, 126.6, 122.5, 119.8, 112.5, 109.8, 105.1, 101.7, 93.9, 62.9, 56.4, 49.3, $45.2,26.8,25.7,24.7,24.4,19.1$, and 18.0.

HRMS (ESI) $m / z:[\mathrm{M}+\mathrm{H}]^{+}$Calcd for $\mathrm{C}_{41} \mathrm{H}_{48} \mathrm{NO}_{8} \mathrm{Si}^{+} 710.3144$; Found 710.3123.

IR (neat): 3071 (br), 3050, 2956, 1761, 1660, and $1602 \mathrm{~cm}^{-1}$. 


\section{4-((tert-Butyldimethylsilyl)oxy)-2-hydroxy-6-methylbenzaldehyde (S13)}<smiles>Cc1cc(O)cc(O)c1C=O</smiles>

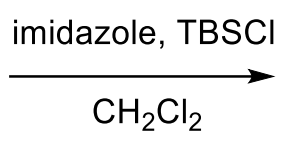<smiles>Cc1cc(O[Sb])cc(O)c1C=O</smiles>

S13

To a stirred solution of the 2,4-dihydroxy-6-methylbenzaldehyde ${ }^{11}$ (650 mg, $\left.4.28 \mathrm{mmol}\right)$ in $\mathrm{CH}_{2} \mathrm{Cl}_{2}(10 \mathrm{~mL})$ were added imidazole $(436 \mathrm{mg}, 6.41 \mathrm{mmol})$ and $t$-butyldimethylchlorosilane $(770$ $\mathrm{mg}, 5.13 \mathrm{mmol}$ ). The resulting mixture was stirred at room temperature for $1 \mathrm{~h}$ before it was quenched by the addition of $\mathrm{NH}_{4} \mathrm{Cl}(10 \mathrm{~mL}$, satd aq). The layers were separated and the aqueous layer was extracted with $\mathrm{CH}_{2} \mathrm{Cl}_{2}(3 \times 20 \mathrm{~mL})$. The combined organic layers were washed with brine $(20 \mathrm{~mL})$, dried $\left(\mathrm{Na}_{2} \mathrm{SO}_{4}\right)$, and concentrated in vacuo. Flash column chromatography (silica gel, hexanes:EtOAc 10:1) of the residue afforded $\mathbf{S 1 3}$ (1000 mg, 90\%) as a colorless oil.

${ }^{1} \mathbf{H}$ NMR (500 MHz, $\left.\mathrm{CDCl}_{3}\right): \delta 12.29$ (s, 1H, $\left.\mathrm{ArOH}\right), 10.10$ (s, 1H, $\left.\mathrm{ArCHO}\right), 6.22(\mathrm{~d}, J=2.4 \mathrm{~Hz}$, $1 \mathrm{H}, \mathrm{ArH3}), 6.21(\mathrm{dq}, J=2.5,0.8 \mathrm{~Hz}, 1 \mathrm{H}, \mathrm{ArH5}), 2.51$ (s, 3H, $\mathrm{ArCH}$ ), 0.98 [s, 9H, C(CH3)3], and $0.25\left[\mathrm{~s}, 6 \mathrm{H}, \mathrm{Si}\left(\mathrm{CH}_{3}\right)_{2}\right]$.

${ }^{13} \mathrm{C} \mathrm{NMR}\left(125 \mathrm{MHz}, \mathrm{CDCl}_{3}\right): \delta 193.2,166.1,163.9,144.2,115.3,114.1,105.9,25.7,18.38,18.35$, and -4.19 .

HRMS (ESI) $m / z$ : $[\mathrm{M}+\mathrm{H}]^{+}$Calcd for $\mathrm{C}_{14} \mathrm{H}_{23} \mathrm{O}_{3} \mathrm{Si}^{+}$267.1411; Found 267.1401.

IR (neat): 2958, 2930, 2861, 1631, and $1567 \mathrm{~cm}^{-1}$. 
4-((tert-Butyldimethylsilyl)oxy)-2-hydroxy-6-methylbenzoic acid (25c)<smiles>Cc1cc(O[Sb])cc(O)c1C=O</smiles>

S13

$$
\underset{\mathrm{H}_{2} \mathrm{O} / \mathrm{THF} / \mathrm{DMSO}}{\stackrel{\mathrm{NH}_{2} \mathrm{SO}_{3} \mathrm{H}, \mathrm{NaClO}_{2}}{\longrightarrow}}
$$<smiles>Cc1cc(O[Sb])cc(O)c1C(=O)O</smiles>

$25 c$

To a stirred solution of $\mathbf{S 1 3}(500 \mathrm{mg}, 1.88 \mathrm{mmol})$ in a cosolvent mixture of $\mathrm{H}_{2} \mathrm{O}(5 \mathrm{~mL})$, THF (2.5 $\mathrm{mL})$, and DMSO $(0.5 \mathrm{~mL})$ were added sulfamic acid $(638 \mathrm{mg}, 6.58 \mathrm{mmol})$ and $\mathrm{NaClO}_{2}(687 \mathrm{mg}$, $6.11 \mathrm{mmol})$. The resulting mixture was stirred at room temperature for $1 \mathrm{~h}$ before it was quenched by the addition of $\mathrm{NH}_{4} \mathrm{Cl}(10 \mathrm{~mL}$, satd aq). The layers were separated and the aqueous layer was extracted with ethyl acetate $(3 \times 10 \mathrm{~mL})$. The combined organic layers were washed with brine $(20$ $\mathrm{mL}$ ), dried $\left(\mathrm{Na}_{2} \mathrm{SO}_{4}\right)$, and concentrated in vacuo. Flash column chromatography (silica gel, hexanes:EtOAc 5:1) of the residue afforded the acid 25c (461 mg, 87\%) as a white crystalline powder.

${ }^{1}$ H NMR (500 MHz, $\left.\mathrm{CDCl}_{3}\right): \delta 11.32(\mathrm{~s}, 1 \mathrm{H}, \mathrm{ArOH})$, ca. $11.3\left(\mathrm{v}\right.$ br s, $\left.1 \mathrm{H}, \mathrm{CO}_{2} H\right), 6.31(\mathrm{~d}, J=2.5$ $\mathrm{Hz}, 1 \mathrm{H}, \mathrm{ArH3}), 6.27(\mathrm{dq}, J=2.5,0.9 \mathrm{~Hz}, 1 \mathrm{H}, \mathrm{ArH5}), 2.56(\mathrm{~s}, 3 \mathrm{H}, \mathrm{ArCH}), 0.98$ [s, 9H, C(CH3)3], and $0.25\left[\mathrm{~s}, 6 \mathrm{H}, \mathrm{Si}\left(\mathrm{CH}_{3}\right)_{2}\right]$.

${ }^{13} \mathrm{C}$ NMR (125 MHz, $\left.\mathrm{CDCl}_{3}\right): \delta 176.3,166.1,161.9,145.0,116.5,105.9,104.8,25.6,24.3,18.2$, and -4.34 .

HRMS (ESI) $m / z:[\mathrm{M}+\mathrm{H}]^{+}$Calcd for $\mathrm{C}_{14} \mathrm{H}_{23} \mathrm{O}_{4} \mathrm{Si}^{+}$283.1360; Found 283.1350.

IR (neat): 3200-3000 (br), 2951, 2927, 2857, 1636, 1613, and $1572 \mathrm{~cm}^{-1}$.

mp: $153-156^{\circ} \mathrm{C}$. 
2-(2-((tert-Butyldiphenylsilyl)oxy)ethyl)-7-(dimethylsilyl)-6-(3-methylbut-2-en-1-yl)-3oxoisoindolin-5-yl 4-((tert-Butyldimethylsilyl)oxy)-2-hydroxy-6-methylbenzoate (27b)

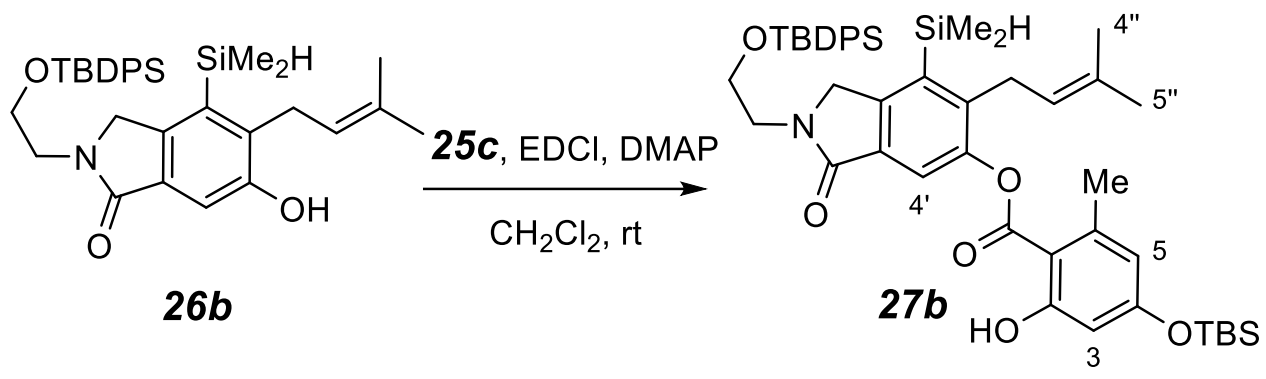

To a stirred solution of the phenol $26 \mathbf{b}(150 \mathrm{mg}, 0.27 \mathrm{mmol})$ in $\mathrm{CH}_{2} \mathrm{Cl}_{2}(10 \mathrm{~mL})$ at $0{ }^{\circ} \mathrm{C}$ was added EDCI $(61.8 \mathrm{mg}, 0.32 \mathrm{mmol})$ followed immediately by a solution of the acid $25 \mathrm{c}(90 \mathrm{mg}, 0.32$ mmol) and DMAP ( $3 \mathrm{mg}, 0.03 \mathrm{mmol})$ in $\mathrm{CH}_{2} \mathrm{Cl}_{2}(5 \mathrm{~mL})$ dropwise over ca. $5 \mathrm{~min}$. The resulting mixture was stirred at room temperature for $1 \mathrm{~h}$ and $\mathrm{NH}_{4} \mathrm{Cl}(10 \mathrm{~mL}$, satd aq) was added. The layers were separated and the aqueous layer was extracted with $\mathrm{CH}_{2} \mathrm{Cl}_{2}(3 \times 10 \mathrm{~mL})$. The combined organic layers were washed with brine $(20 \mathrm{~mL})$, dried $\left(\mathrm{Na}_{2} \mathrm{SO}_{4}\right)$, and concentrated in vacuo. Flash column chromatography (silica gel, hexanes:EtOAc 10:1) afforded the ester $\mathbf{2 7 b}$ (175 $\mathrm{mg}, \mathbf{7 8 \%}$ ) as a white crystalline solid.

${ }^{1} \mathbf{H}$ NMR (500 MHz, CDCl 3$): \delta 11.33$ (s, 1H, ArOH), 7.62 (2 overlapping app d, $J$ ca. $7 \mathrm{~Hz}, 4 \mathrm{H}$, $\mathrm{Ph} H_{o}$ ), 7.57 (s, $1 \mathrm{H}, \mathrm{Ar} H 4$ '), 7.41 (two perfectly overlapped tt, $J=7.4,1.3 \mathrm{~Hz}, 2 \mathrm{H}, \mathrm{Ph} H_{p}$ ), 7.33 (2 overlapping app d, $J$ ca. $\left.7 \mathrm{~Hz}, 4 \mathrm{H}, \mathrm{Ph} H_{m}\right), 6.34$ (d, $J=2.5 \mathrm{~Hz}, 1 \mathrm{H}, \mathrm{ArH3}$ ), 6.31 (br d, $J=2.5 \mathrm{~Hz}$, $1 \mathrm{H}, \mathrm{ArH5}$ ), 4.95 (tqq, $\left.J=6.0,1.4,1.4 \mathrm{~Hz}, 1 \mathrm{H}, \mathrm{C}=\mathrm{CHCH}_{2}\right), 4.67$ (s, 2H, $\mathrm{ArCH}_{2} \mathrm{~N}$ ), 4.58 [sept, $J=$ $4.0 \mathrm{~Hz}, 1 \mathrm{H}, \mathrm{SiH}\left(\mathrm{CH}_{3}\right)_{2}$ ], $3.93\left(\operatorname{app~t}, J=4.9 \mathrm{~Hz}, 2 \mathrm{H}, \mathrm{OCH}_{2} \mathrm{CH}_{2}\right), 3.77$ (app t, $J=5.0 \mathrm{~Hz}, 2 \mathrm{H}$, $\mathrm{OCH}_{2} \mathrm{CH}_{2}$ ), 3.49 (nfom, 2H, $\mathrm{ArCH}_{2} \mathrm{CH}=\mathrm{C}$ ), 2.63 (br s, 3H, $\mathrm{ArCH}$ ), 1.56 (br d, J = 1.4 Hz, 3H, C5 ' 'H3), 1.42 (br d, $\left.J=1.4 \mathrm{~Hz}, 3 \mathrm{H}, \mathrm{C} 4{ }^{\prime} \mathrm{H}_{3}\right), 1.07$ [s, 9H, $\left.\mathrm{SiC}\left(\mathrm{CH}_{3}\right)_{3}\right], 1.00$ [s, 9H, $\left.\mathrm{SiC}\left(\mathrm{CH}_{3}\right)_{3}\right]$, $0.39\left[\mathrm{~d}, J=4.0 \mathrm{~Hz}, 6 \mathrm{H}, \mathrm{SiH}\left(\mathrm{CH}_{3}\right)_{2}\right]$, and 0.27 [s, $\left.6 \mathrm{H}, \mathrm{Si}\left(\mathrm{CH}_{3}\right)_{2}\right]$.

${ }^{13} \mathrm{C}$ NMR (125 MHz, $\left.\mathrm{CDCl}_{3}\right): \delta 170.8,167.7,166.0,161.6,149.1,146.1,143.8,143.4,135.6$, $133.5,133.2$, 133.1 131.9, 130.0, 127.9, 122.0, 119.4, 116.6, 106.1, 105.5, 63.7, 54.0, 45.1, 30.9, $27.0,25.7,25.5,24.6,19.3,18.4,17.9,-2.6$, and -4.2 .

HRMS (ESI) $m / z:[\mathrm{M}+\mathrm{H}]^{+}$Calcd for $\mathrm{C}_{47} \mathrm{H}_{64} \mathrm{NO}_{6} \mathrm{Si}_{3}{ }^{+}$822.4036; Found 822.4018.

IR (neat): 3074 (br), 3055, 2955, 2930, 2861, 2130, 1694, 1663, 1614, and $1569 \mathrm{~cm}^{-1}$.

mp: $56-58^{\circ} \mathrm{C}$. 
2-(2-((tert-Butyldiphenylsilyl)oxy)ethyl)-7-(hydroxydimethylsilyl)-6-(3-methylbut-2-en-1yl)-3-oxoisoindolin-5-yl 4-((tert-butyldimethylsilyl)oxy)-2-hydroxy-6-methylbenzoate (28)

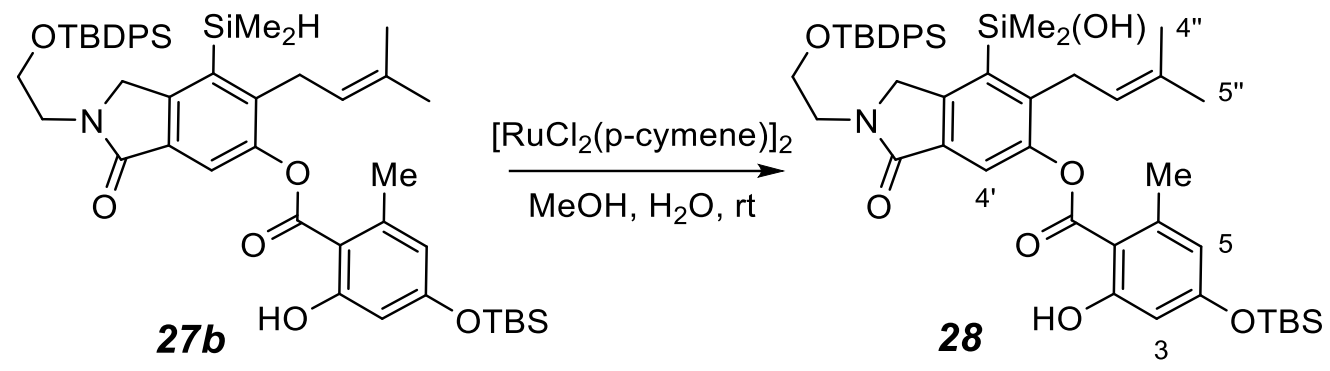

To a stirred solution of $\mathbf{2 7 b}(80 \mathrm{mg}, 0.097 \mathrm{mmol})$ in $\mathrm{MeOH}(3 \mathrm{~mL})$ and $\mathrm{H}_{2} \mathrm{O}(3 \mathrm{~mL})$ was added $\left[\mathrm{RuCl}_{2}(p \text {-cymene })\right]_{2}(3 \mathrm{mg}, 0.005 \mathrm{mmol})$. The resulting mixture was stirred at room temperature for $30 \mathrm{~min}$ before it was quenched by the addition of $\mathrm{NaHCO}_{3}(10 \mathrm{~mL}$, satd aq). The layers were separated and the aqueous layer was extracted with $\mathrm{CH}_{2} \mathrm{Cl}_{2}(3 \times 10 \mathrm{~mL})$. The combined organic layers were washed with brine $(15 \mathrm{~mL})$, dried $\left(\mathrm{Na}_{2} \mathrm{SO}_{4}\right)$, and concentrated in vacuo. Flash column chromatography (silica gel, hexanes:EtOAc 3:1) of the residue afforded the silanol 28 (70 $\mathrm{mg}$, $85 \%$ ) as a white crystalline solid.

${ }^{1}$ H NMR (500 MHz, $\left.\mathrm{CDCl}_{3}\right): \delta 11.33$ (s, 1H, ArOH), 7.60 (2 overlapping app d, $J$ ca. $7 \mathrm{~Hz}, 4 \mathrm{H}$, $\mathrm{Ph} H_{o}$ ), 7.57 (s, 1H, ArH4'), 7.40 (two perfectly overlapped tt, $J=7.4,1.4 \mathrm{~Hz}, 2 \mathrm{H}, \mathrm{Ph} H_{p}$ ), 7.33 (2 overlapping app t, $J$ ca. $\left.7 \mathrm{~Hz}, 4 \mathrm{H}, \mathrm{Ph} H_{m}\right), 6.34$ (d, $\left.J=2.5 \mathrm{~Hz}, 1 \mathrm{H}, \mathrm{ArH3}\right), 6.30$ (br d, $J=2.5 \mathrm{~Hz}$, $1 \mathrm{H}, \mathrm{ArH5}), 4.94\left(\mathrm{tqq}, J=6.0,1.4,1.4 \mathrm{~Hz}, 1 \mathrm{H}, \mathrm{C}=\mathrm{CHCH}_{2}\right), 4.74\left(\mathrm{~s}, 2 \mathrm{H}, \mathrm{ArCH}_{2} \mathrm{~N}\right), 3.93(\operatorname{app~t}, J$ $\left.=5.0 \mathrm{~Hz}, 2 \mathrm{H}, \mathrm{OCH}_{2} \mathrm{CH}_{2}\right), 3.73\left(\operatorname{app} \mathrm{t}, J=5.0 \mathrm{~Hz}, 2 \mathrm{H}, \mathrm{OCH}_{2} \mathrm{CH}_{2}\right), 3.47-3.44(\mathrm{~m}, 2 \mathrm{H}$, $\left.\mathrm{ArCH}_{2} \mathrm{CH}=\mathrm{C}\right), 2.61$ (s, 3H, $\left.\mathrm{ArCH}_{3}\right), 1.54$ (dt, $J=1.6,1.6 \mathrm{~Hz}, 3 \mathrm{H}, \mathrm{C} 5$ ' ' $\left.\mathrm{H}_{3}\right), 1.40$ (br d, $J=1.3 \mathrm{~Hz}$, $\left.3 \mathrm{H}, \mathrm{C} 4{ }^{\prime \prime} \mathrm{H}_{3}\right), 1.06$ [s, 9H, $\left.\mathrm{SiC}\left(\mathrm{CH}_{3}\right)_{3}\right], 1.00$ [s, 9H, $\left.\mathrm{SiC}\left(\mathrm{CH}_{3}\right)_{3}\right], 0.45$ [s, 6H, $\left.\mathrm{HOSi}\left(\mathrm{CH}_{3}\right)_{2}\right]$, and 0.26 [s, 6H, $\left.\mathrm{Si}\left(\mathrm{CH}_{3}\right)_{2}\right]$.

${ }^{13} \mathrm{C}$ NMR (125 MHz, $\left.\mathrm{CDCl}_{3}\right): \delta$ 170.8, 167.5, 166.0, 161.6, 149.2, 145.7, 143.79, 142.4, 135.6, 135.6, 135.2, 133.5, 132.3, 130.0, 127.9, 122.2, 119.4, 116.6, 106.1, 105.6, 63.5, 54.6, 45.1, 30.8, $27.0,25.7,25.4,24.6,19.5,18.4,17.9,3.1$, and -4.18 .

HRMS (ESI) $m / z:[\mathrm{M}+\mathrm{H}]^{+}$Calcd for $\mathrm{C}_{41} \mathrm{H}_{48} \mathrm{NO}_{8} \mathrm{Si}^{+}$838.3985; Found 838.3968.

IR (neat): 3071 (br), 3052, 2958, 2930, 2858, 1663, and $1615 \mathrm{~cm}^{-1}$. mp: $53-56{ }^{\circ} \mathrm{C}$. 


\section{Sterenin A (3)}

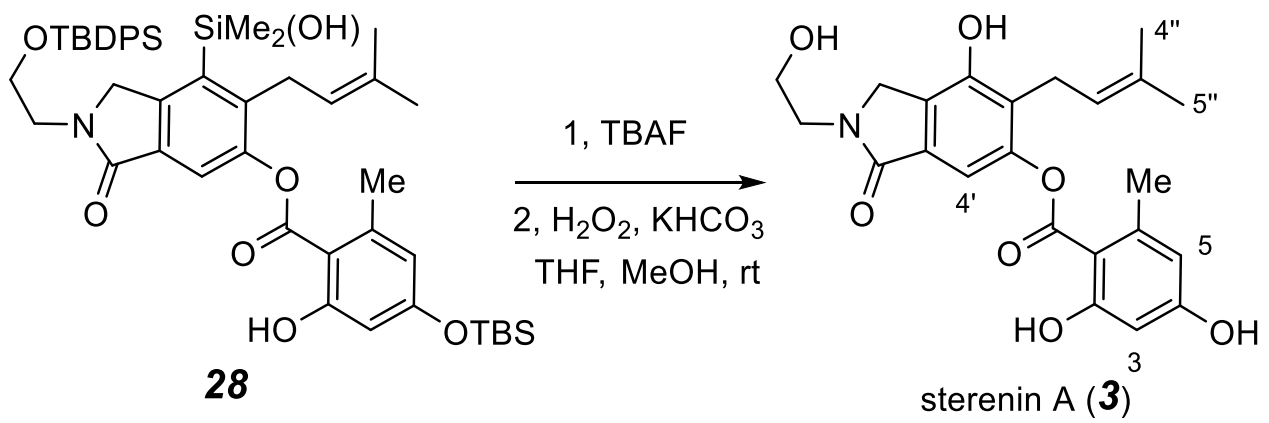

To a stirred solution of 28 (25 mg, $0.03 \mathrm{mmol})$ in THF (1.5 mL) was added TBAF (1.0 M in THF, $1200 \mu \mathrm{L}, 0.06 \mathrm{mmol})$. The resulting mixture was stirred at room temperature for $30 \mathrm{~min}$. $\mathrm{MeOH}$ ( $1 \mathrm{~mL}$ ), $\mathrm{KHCO}_{3}\left(3 \mathrm{mg}, 0.03 \mathrm{mmol}\right.$ ), and $\mathrm{H}_{2} \mathrm{O}_{2}$ (30 wt. $\%$ in $\mathrm{H}_{2} \mathrm{O}, 36 \mu \mathrm{L}, 0.3 \mathrm{mmol}$ ) were added. The resulting mixture was stirred at room temperature for $4 \mathrm{~h}$, and $\mathrm{NaHCO}_{3}(5 \mathrm{~mL}$, satd aq) was added. The layers were separated and the aqueous layer was extracted with ethyl acetate (3x10 $\mathrm{mL})$. The combined organic layers were washed with brine $(10 \mathrm{~mL})$, dried $\left(\mathrm{Na}_{2} \mathrm{SO}_{4}\right)$, and concentrated in vacuo. Flash column chromatography (silica gel, hexanes:EtOAc 1:10) of the residue afforded sterenin $\mathrm{A}(\mathbf{3})(10 \mathrm{mg}, 80 \%)$ as a white powder.

${ }^{1} \mathbf{H}$ NMR (500 MHz, CD $\left.3 \mathrm{OD}\right): \delta 7.07$ (s, 1H, ArH4'), 6.30 (dq, $\left.J=2.5,0.9 \mathrm{~Hz}, 1 \mathrm{H}, \mathrm{ArH5}\right), 6.22$ $(\mathrm{dq}, J=2.5,0.5 \mathrm{~Hz}, 1 \mathrm{H}, \mathrm{ArH3}), 5.03\left(\mathrm{tqq}, J=6.0,1.4,1.4 \mathrm{~Hz}, 1 \mathrm{H}, \mathrm{C}=\mathrm{CHCH}_{2}\right), 4.58(\mathrm{~s}, 2 \mathrm{H}$, $\mathrm{ArCH}_{2} \mathrm{~N}$ ), $3.83\left(\operatorname{app~t}, J=5.0 \mathrm{~Hz}, 2 \mathrm{H}, \mathrm{OCH}_{2} \mathrm{CH}_{2}\right), 3.74\left(\operatorname{app~t}, J=5.0 \mathrm{~Hz}, 2 \mathrm{H}, \mathrm{OCH}_{2} \mathrm{CH}_{2}\right), 3.38$ $\left(\mathrm{dqq}, J=6.8,1.2,1.2 \mathrm{~Hz}, 2 \mathrm{H}, \mathrm{ArCH}_{2} \mathrm{CH}=\mathrm{C}\right), 2.60\left(\mathrm{dd}, J=0.9,0.5 \mathrm{~Hz}, 3 \mathrm{H}, \mathrm{ArCH}_{3}\right), 1.56(\mathrm{dt}, J=$ 1.6, $\left.1.6 \mathrm{~Hz}, 3 \mathrm{H}, \mathrm{C} 5{ }^{\prime \prime} H_{3}\right)$, and $1.50\left(\mathrm{brd}, J=1.2 \mathrm{~Hz}, 3 \mathrm{H}, \mathrm{C} 4{ }^{\prime \prime} H_{3}\right)$.

${ }^{13} \mathrm{C}$ NMR (125 MHz, CD $\left.3 \mathrm{OD}\right): \delta 171.8,170.7,167.1,164.9,152.2,151.4,145.1,133.3,132.8$, $127.2,127.1,122.7,113.1,110.1,105.3,102.0,61.3,50.9,46.5,25.9,24.72,24.69$, and 17.9.

HRMS (ESI) $m / z:[\mathrm{M}+\mathrm{H}]^{+}$Calcd for $\mathrm{C}_{23} \mathrm{H}_{26} \mathrm{NO}_{7}{ }^{+}$428.1704; Found 428.1696.

IR (neat): 3350, 2964, 2931, 2854, 1652, and $1613 \mathrm{~cm}^{-1}$.

mp: $161.5-164{ }^{\circ} \mathrm{C}\left(\right.$ lit. $\left.^{10} 162-164{ }^{\circ} \mathrm{C}\right)$. 
IIIa. Tabulated NMR spectral data for comparison of reported vs. this work

Isohericerin (1)

\begin{tabular}{|c|c|}
\hline $\begin{array}{l}\text { M. Miyazawa et al. } 2012 \\
\left(500 / 125 \mathrm{MHz}, \mathrm{CDCl}_{3}\right)^{5}\end{array}$ & $\begin{array}{c}\text { C. Zhu, J. Zhang, T. Hoye } \\
\text { (this report, } 500 / 125 \mathrm{MHz}, \mathrm{CDCl}_{3} \text { ) }\end{array}$ \\
\hline$\delta_{H}(J$ in $\mathrm{Hz})$ & $\delta_{H}(J$ in $\mathrm{Hz})$ \\
\hline $7.26 \mathrm{dd}(7.2,7.2)$ & $7.26 \mathrm{nfom}$ \\
\hline $7.22 \mathrm{dd}(7.2,1.7)$ & 7.22 nfom \\
\hline $7.19 \mathrm{dd}(7.2,1.7)$ & $7.19 \mathrm{tt}(7.1,1.4)$ \\
\hline $6.95 \mathrm{~s}$ & $6.96 \mathrm{~s}$ \\
\hline $6.52 \mathrm{~s}$ & $6.31 \mathrm{br} \mathrm{s}(\mathrm{ArOH})$ \\
\hline $5.24 \mathrm{brt}(6.8)$ & $5.24 \mathrm{dqt}(7.1,1.3,1.3)$ \\
\hline $5.03 \mathrm{brt}(6.8)$ & $5.03 \mathrm{dqq}(6.8,1.4,1.4)$ \\
\hline 3.84 br t (7.2) & 3.84 nfot \\
\hline $3.83 \mathrm{~s}$ & $3.83 \mathrm{~s}$ \\
\hline $3.49 \mathrm{~d}(6.8)$ & $3.49 \mathrm{dqt}(7.2,1.0,1.0)$ \\
\hline $2.97 \mathrm{t}(7.2)$ & 2.97 nfot \\
\hline $2.10 \mathrm{~m}$ & $2.10 \mathrm{br} d t(7.0,7.0)$ \\
\hline $2.05 \mathrm{~m}$ & $2.06 \mathrm{brt}(7.0)$ \\
\hline $1.80 \mathrm{~s}$ & $1.81 \mathrm{dt}(1.1,1.1)$ \\
\hline $1.66 \mathrm{~s}$ & $1.66 \mathrm{dt}(1.4,1.4)$ \\
\hline $1.58 \mathrm{~s}$ & $1.58 \mathrm{br} \mathrm{d}(1.4)$ \\
\hline
\end{tabular}

\begin{tabular}{|c|c|c|}
\hline M. Miyazawa et al. ${ }^{5}$ & K. R. Lee et al. $2012^{12}$ & $\begin{array}{l}\text { C. Zhu, J. Zhang, T. Hoye } \\
\text { (this report) }\end{array}$ \\
\hline$\delta_{c}$ & $\delta_{c}$ & $\delta_{c}$ \\
\hline 169.0 & 169.3 & 169.1 \\
\hline 158.4 & 158.5 & 158.6 \\
\hline 150.4 & 150.6 & 150.7 \\
\hline 139.1 & 138.8 & 139.5 \\
\hline 138.7 & 138.8 & 138.9 \\
\hline 132.1 & 132.1 & 132.30 \\
\hline 132.0 & 132.0 & 132.29 \\
\hline 128.6 & 128.8 & 128.8 \\
\hline 128.6 & 128.7 & 128.7 \\
\hline 126.4 & 126.7 & 126.6 \\
\hline 123.7 & 124.0 & 123.8 \\
\hline 121.1 & 121.5 & 121.4 \\
\hline no second peak reported & 121.3 & 121.3 \\
\hline 118.1 & 119.4 & 118.6 \\
\hline 97.5 & 97.8 & 97.7 \\
\hline 56.0 & 56.1 & 56.2 \\
\hline 48.2 & 48.5 & 48.3 \\
\hline 44.2 & 44.2 & 44.4 \\
\hline 39.6 & 39.9 & 39.8 \\
\hline 34.8 & 35.0 & 35.0 \\
\hline 26.3 & 26.5 & 26.4 \\
\hline 25.7 & 25.8 & 25.8 \\
\hline 22.8 & 23.0 & 22.9 \\
\hline 22.8 & no second peak reported & no second peak observed \\
\hline 17.7 & 17.8 & 17.8 \\
\hline 16.1 & 16.3 & 16.3 \\
\hline
\end{tabular}




\begin{tabular}{|c|c|}
\hline $\begin{array}{c}\text { M. Kikuchi et al. }{ }^{13} \\
\left(400 / 100 \mathrm{MHz}, \mathrm{CDCl}_{3}\right)\end{array}$ & $\begin{array}{c}\text { C. Zhu, J. Zhang, T. Hoye } \\
\text { (this report, 500/125 MHz, } \mathrm{CDCl}_{3} \text { ) }\end{array}$ \\
\hline$\delta_{H}(J$ in $\mathrm{Hz})$ & $\delta_{H}(J$ in $\mathrm{Hz})$ \\
\hline \multirow{3}{*}{$7.32-7.20 \mathrm{~m}$} & $7.31-7.28 \mathrm{~m}$ \\
\hline & $7.25-7.21 \mathrm{~m}$ \\
\hline & $7.20 \mathrm{tt},(7.1,1.4)$ \\
\hline $6.90 \mathrm{~s}$ & $6.90 \mathrm{~s}$ \\
\hline $6.05 \mathrm{brs}$ & $6.05 \mathrm{qq}(1.2,1.2)$ \\
\hline $4.17 \mathrm{~d}(16.8)$ & $4.16 \mathrm{~d}(17)$ \\
\hline $4.11 \mathrm{~d}(16.8)$ & $4.12 \mathrm{~d}(16.8)$ \\
\hline $3.87 \mathrm{~s}$ & $3.87 \mathrm{~s}$ \\
\hline $3.84 t(7.6)+3.83 t(7.6)$ & $3.84 \mathrm{nfot}$ \\
\hline $2.98 \mathrm{t}(7.6)$ & $2.98 \mathrm{nfot}$ \\
\hline $2.76 \mathrm{~d}(14.1)$ & $2.76 \mathrm{~d}(14.1)$ \\
\hline $2.69 \mathrm{dd}(6.8,6.6)+2.69 \mathrm{dd}(7.1,6.6)$ & 2.73-2.67 nfom \\
\hline $2.64 \mathrm{~d}(14.1)$ & $2.64 \mathrm{~d}(14.4)$ \\
\hline $2.14 \mathrm{~d}(1.2)$ & $2.15 \mathrm{~d}(1.2)$ \\
\hline $2.02 \mathrm{ddd}(13.9,6.6,6.6)$ & $2.02 \mathrm{dt}(14,6.6)$ \\
\hline $1.90 \mathrm{ddd}(13.9,7.1,6.8)$ & $1.90 \mathrm{dt}(14,6.9)$ \\
\hline $1.85 \mathrm{~d}(1.2)$ & $1.85 \mathrm{~d}(1.2)$ \\
\hline $1.41 \mathrm{~s}$ & $1.41 \mathrm{~s}$ \\
\hline$\delta c$ & $\delta c$ \\
\hline 198.1 & 198.2 \\
\hline 168.9 & 169.1 \\
\hline 158.5 & 158.6 \\
\hline 155.6 & 155.8 \\
\hline 148.6 & 148.7 \\
\hline 138.8 & 138.9 \\
\hline 132.2 & 132.4 \\
\hline 128.7 & 128.9 \\
\hline 128.6 & 128.8 \\
\hline 126.5 & 126.6 \\
\hline 125.0 & 125.2 \\
\hline 121.9 & 122.0 \\
\hline 113.5 & 113.7 \\
\hline 96.2 & 96.3 \\
\hline 75.9 & 76.1 \\
\hline 55.9 & 56.0 \\
\hline 52.3 & 52.4 \\
\hline 48.0 & 48.1 \\
\hline 44.4 & 44.3 \\
\hline 34.9 & 35.1 \\
\hline 30.6 & 30.8 \\
\hline 27.8 & 28.0 \\
\hline 24.8 & 24.9 \\
\hline 20.8 & 20.9 \\
\hline 17.4 & 17.5 \\
\hline
\end{tabular}


IIIc. Tabulated NMR spectral data for comparison of reported vs. this work

Sterenin A (3)

\begin{tabular}{|c|c|}
\hline $\begin{array}{c}\text { T. Takatsu et al. }{ }^{14} \\
\left(500 / 125 \mathrm{MHz}, \mathrm{CD}_{3} \mathrm{OD}\right)\end{array}$ & $\begin{array}{c}\text { C. Zhu, J. Zhang, T. Hoye } \\
\text { (this report, } 500 / 125 \mathrm{MHz}, \mathrm{CD}_{3} \mathrm{OD} \text { ) }\end{array}$ \\
\hline$\delta_{H}(J$ in $H z)$ & $\delta_{H}(J$ in $H z)$ \\
\hline $7.08 \mathrm{~s}$ & $7.07 \mathrm{~s}$ \\
\hline $6.31 \mathrm{brs}$ & $6.30 \mathrm{dq}(2.5,0.9)$ \\
\hline $6.23 \mathrm{br} \mathrm{s}$ & $6.22 \mathrm{dq}(2.5,0.5)$ \\
\hline $5.03 \mathrm{~m}$ & 5.03 tqq $(6.0,1.4,1.4)$ \\
\hline $4.58 \mathrm{~s}$ & $4.58 \mathrm{~s}$ \\
\hline 3.83 br t (5.1) & 3.83 app t (5.0) \\
\hline $3.75 \mathrm{t}(5.1)$ & 3.74 app t (5.0) \\
\hline $3.37 \mathrm{~m}$ & $3.38 \mathrm{dqq}(6.8,1.2,1.2)$ \\
\hline $2.60 \mathrm{~s}$ & $2.60 \mathrm{dd}(0.9,0.5)$ \\
\hline $1.56 \mathrm{~s}$ & $1.56 \mathrm{dt}(1.6,1.6)$ \\
\hline $1.50 \mathrm{~s}$ & $1.50 \mathrm{brd}(1.2)$ \\
\hline
\end{tabular}

\begin{tabular}{|c|c|}
\hline$\delta_{c}$ & $\delta_{c}$ \\
\hline 171.8 & 171.8 \\
\hline 170.7 & 170.7 \\
\hline 167.1 & 167.1 \\
\hline 164.9 & 164.9 \\
\hline 152.4 & 152.2 \\
\hline 151.3 & 151.4 \\
\hline 145.1 & 145.1 \\
\hline 133.3 & 133.3 \\
\hline 132.7 & 132.8 \\
\hline 127.2 & 127.2 \\
\hline 127.1 & 127.1 \\
\hline 122.6 & 122.7 \\
\hline 113.1 & 113.1 \\
\hline 110.1 & 110.1 \\
\hline 105.3 & 105.3 \\
\hline 102.0 & 102.0 \\
\hline 61.3 & 61.3 \\
\hline 50.9 & 50.9 \\
\hline 46.5 & 46.5 \\
\hline 25.9 & 25.9 \\
\hline \multirow{2}{*}{24.7} & 24.72 \\
\hline & 24.69 \\
\hline 17.9 & 17.9 \\
\hline
\end{tabular}




\section{Computational methods}

DFT computations were performed with the Gaussian 09 software package. ${ }^{15}$ The geometries were optimized with the M06-2X functional; ${ }^{16}$ the basis set was double- $\zeta$ split-valence $6-311+\mathrm{G}(\mathrm{d}$, p). The SMD continuum solvation model ${ }^{17}$ with methanol as solvent was applied during both the frequency calculation and the geometry optimization. Harmonic vibrational frequency calculations were performed at $298 \mathrm{~K}$ and used for the thermal correction of enthalpies. The value for the "Sum of electronic and thermal Free Energies=" was used to as the free energy (G) of the transition state structures $\left(\mathrm{G}_{\mathrm{TS}}\right)$ as well as of the reactants and products. The optimized transition state structure geometry contained only one imaginary frequency.

\section{Geometry for the model benzyne (9)}

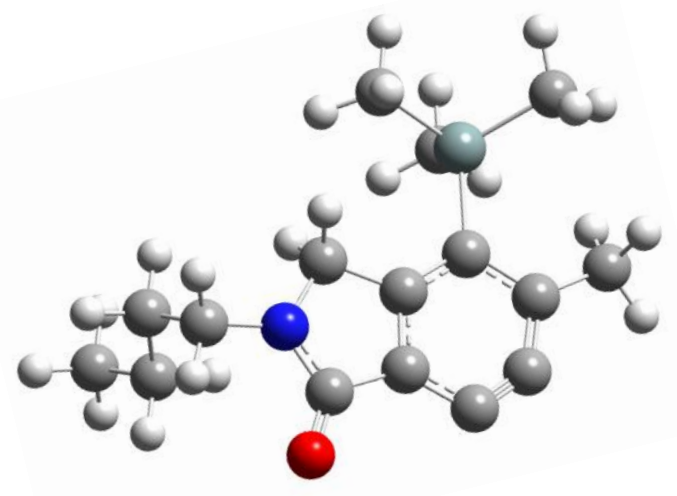

\begin{tabular}{|c|c|c|c|c|c|}
\hline \multirow{2}{*}{$\begin{array}{l}\text { Center } \\
\text { Number }\end{array}$} & \multirow{2}{*}{$\begin{array}{l}\text { Atomic } \\
\text { Number }\end{array}$} & \multirow{2}{*}{$\begin{array}{c}\text { Atomic } \\
\text { Type }\end{array}$} & \multicolumn{3}{|c|}{ Coordinates (Angstroms) } \\
\hline & & & $\mathrm{X}$ & $\mathrm{Y}$ & \\
\hline 1 & 6 & 0 & -2.349145 & 1.540209 & 0.593846 \\
\hline 2 & 6 & 0 & -1.517848 & 2.637377 & 0.658696 \\
\hline 3 & 6 & 0 & -0.328194 & 2.872955 & 0.387048 \\
\hline 4 & 6 & 0 & 0.431175 & 1.833503 & -0.129765 \\
\hline 5 & 6 & 0 & -0.261529 & 0.620045 & -0.291426 \\
\hline 6 & 6 & 0 & -1.619686 & 0.438497 & 0.042508 \\
\hline 7 & 6 & 0 & 1.851069 & 1.678159 & -0.531804 \\
\hline 8 & 8 & 0 & 2.735176 & 2.523645 & -0.503355 \\
\hline 9 & 7 & 0 & 1.970165 & 0.389375 & -0.938864 \\
\hline 10 & 6 & 0 & -3.783067 & 1.517090 & 1.037842 \\
\hline 11 & 1 & 0 & -4.453514 & 1.623204 & 0.181770 \\
\hline 12 & 1 & 0 & -4.031438 & 0.585787 & 1.546572 \\
\hline
\end{tabular}




\begin{tabular}{|c|c|c|c|c|c|}
\hline 13 & 1 & 0 & -3.973048 & 2.343234 & 1.721062 \\
\hline 14 & 6 & 0 & 3.218283 & -0.216622 & -1.368101 \\
\hline 15 & 1 & 0 & 3.939011 & 0.597556 & -1.462106 \\
\hline 16 & 1 & 0 & 3.072898 & -0.659438 & -2.357963 \\
\hline 17 & 6 & 0 & 3.729921 & -1.266454 & -0.381353 \\
\hline 18 & 1 & 0 & 4.688990 & -1.637268 & -0.757165 \\
\hline 19 & 1 & 0 & 3.052883 & -2.128107 & -0.365151 \\
\hline 20 & 6 & 0 & 3.904114 & -0.719703 & 1.033133 \\
\hline 21 & 1 & 0 & 4.563452 & 0.154511 & 1.001037 \\
\hline 22 & 1 & 0 & 2.937723 & -0.364218 & 1.407393 \\
\hline 23 & 6 & 0 & 4.470337 & -1.766065 & 1.989461 \\
\hline 24 & 1 & 0 & 3.811751 & -2.637113 & 2.044194 \\
\hline 25 & 1 & 0 & 5.451265 & -2.110640 & 1.651967 \\
\hline 26 & 1 & 0 & 4.582737 & -1.364954 & 2.998483 \\
\hline 27 & 6 & 0 & 0.741163 & -0.381816 & -0.821576 \\
\hline 28 & 1 & 0 & 0.877798 & -1.214576 & -0.123040 \\
\hline 29 & 1 & 0 & 0.459256 & -0.789690 & -1.795713 \\
\hline 30 & 14 & 0 & -2.510767 & -1.250583 & -0.153624 \\
\hline 31 & 6 & 0 & -1.604203 & -2.403419 & -1.328166 \\
\hline 32 & 1 & 0 & -1.478913 & -1.966835 & -2.321931 \\
\hline 33 & 1 & 0 & -0.633054 & -2.738580 & -0.962264 \\
\hline 34 & 1 & 0 & -2.234917 & -3.291299 & -1.438199 \\
\hline 35 & 6 & 0 & -4.229404 & -1.013709 & -0.882983 \\
\hline 36 & 1 & 0 & -4.553082 & -1.966528 & -1.311425 \\
\hline 37 & 1 & 0 & -4.974457 & -0.711179 & -0.146699 \\
\hline 38 & 1 & 0 & -4.220287 & -0.273861 & -1.687945 \\
\hline 39 & 6 & 0 & -2.588015 & -2.060495 & 1.538088 \\
\hline 40 & 1 & 0 & -3.096424 & -3.026057 & 1.465599 \\
\hline 41 & 1 & 0 & -1.578263 & -2.237130 & 1.918312 \\
\hline 42 & 1 & 0 & -3.122014 & -1.450760 & 2.270138 \\
\hline
\end{tabular}




\section{References for the Supporting Information}

1 (a) Hoye, T. R.; Baire, B.; Niu, D.; Willoughby, P. H.; Woods, B. P. The hexadehydro-Diels-Alder reaction. Nature 2012, 490, 208-212. (b) Wang, K-P.; Yun, S. Y.; Mamidipalli, P.; Lee, D. Chem. Sci. 2013, 4, 3205-3211. (c) Niu, D.; Willoughby, P. H.; Woods, B. P.; Baire, B. Alkane desaturation by concerted double hydrogen atom transfer to benzyne. Nature 2013, 501, 531-534. (d) Niu, D.; Hoye, T. R. The aromatic ene reaction. Nature Chem. 2014, 6, 34-40. (e) Niu, D.; Wang, T.; Woods, B. P.; Hoye, T. R. Dichlorination of (hexadehydro-Diels-Alder generated) benzynes and a protocol for interrogating the kinetic order of bimolecular reactions aryne trapping reactions. Org. Lett. 2014, 16, 254-257. (f) Lee, N-K.; Yun, S. Y.; Mamidipalli, P.; Salzman, R. M.; Lee, D.; Zhou, T.; Xia, Y. Hydroarylation of arynes catalyzed by silver for biaryl synthesis. J. Am. Chem. Soc. 2014, 113, 4363-4368. (g) Woods, B. P.; Baire, B.; Hoye, T. R. Rates of hexadehydro-Diels-Alder (HDDA) cyclizations: Impact of the linker structure. Org. Lett. 2014, 16, 4578-4581. (h) Karmakar, R.; Yun, S. Y.; Chen, J.; Xia, Y.; Lee, D. Benzannulation of triynes to generate functionalized arenes by spontaneous incorporation of nucleophiles. Angew. Chem. Int. Ed. 2015, 54, 6582-6586. (i) Watanabe, T.; Curran, D. P.; Taniguchi, T. Hydroboration of arynes formed by hexadehydro-Diels-Alder cyclizations with N-heterocyclic carbene boranes. Org. Lett. 2015, 17, 3450-3453. (j) Chen, J.; Palani, V.; Hoye, T. R. Reactions of HDDA-derived benzynes with sulfides: Mechanism, and threecomponent reactions. J. Am. Chem. Soc. 2016, 138, 4318-4321. (k) Palani, V.; Chen, J.; Hoye, T. R. Reactions of HDDA-derived benzynes with thioamides: Synthesis of dihydrobenzothiazino-heterocyclics. Org. Lett. 2016, 18, 6312-6315. (1) Gupta, S.; Xie, P.; Xia, Y.; Lee, D. Reactivity and selectivity in the intermolecular Alder-ene reactions of arynes with functionalized alkenes. Org. Lett. 2017, 19, 5162-5165. (m) Ross, S. P.; Hoye, T. R. Multiheterocyclic motifs via three-component reactions of benzynes, cyclic amines, and protic nucleophiles. Org. Lett. 2018, 20, 100-103. (n) Wang, Y.; Zheng, L.; Hoye, T. R. Sulfonamide trapping reactions of thermally generated benzynes. Org. Lett. 2018, 20, 7145-7148. (o) Shen, H.; Xiao, X.; Haj, M.; Willoughby, P. H.; Hoye, T. R. BF 3 -Promoted, carbene-like, C-H insertion reactions of benzynes. J. Am. Chem. Soc. 2018, 140, 1561615620. (p) Gupta, S.; Lin, Y.; Xia, Y.; Wink, D. J.; Lee, D. Alder-ene reactions driven by high steric strain and bond angle distortion to form benzocyclobutenes. Chem. Sci. 2019, 10, 2212-2217. (q) Shen, H.; Xiao, X.; Hoye, T. R. Benzyne cascade reactions via benzoxetenonium ions and their rearrangements to $o$-quinone methides. Org. Lett. 2019, 21, 1672-1675. (r) Ghorai, S.; Lee, D. Synthesis of imides, imidates, amidines, and amides by intercepting the aryne-isocyanide adduct with weak nucleophiles. Org. Lett. 2019, 21, 7390-7393. (s) Ghorai, S.; Lin, Y.; Xia, Y.; Wink, D. J.; Lee, D. Silver-catalyzed annulation of arynes with nitriles for synthesis diverse quinazolines. Org. Lett. 2020, 22, 626-630. (t) Ghorai, S.; Lin, Y.; Xia, Y.; Wink, D. J.; Lee, D. Silver-catalyzed selective multicomponent coupling reactions of arynes with nitriles and isonitriles. Org. Lett. 2020, 22, 642-647.

2 Hoye, T. R.; Hanson, P. R.; Vyvyan, J. R. A practical guide to first-order multiplet analysis in ${ }^{1} \mathrm{H}$ NMR spectroscopy. J. Org. Chem. 1994, 59, 4096-4103.

3 Hoye, T. R.; Zhao, H. A method for easily determining coupling constant values: An addendum to "A practical guide to first-order multiplet analysis in ${ }^{1} \mathrm{H}$ NMR spectroscopy" J. Org. Chem. 2002, 67, 4014-4016.

$4 \quad$ Karaev, S. F.; Kuliev, R. M.; Guseinov, Sh. O.; Askerov, M. E.; Movsumzade, M. M. Synthesis and reactions of dimethylbenzylsilylpropargyl alcohol. Zhurnal Obshchei Khimii 1982, 52, 1160-1163.

5 Miyazawa, M.; Takahashi, T.; Horibe, I.; Ishikawa, R. Two new aromatic compounds and a new D-arabinitol ester from the mushroom Hericium erinaceum. Tetrahedron 2012, 68, 2007-2010.

6 Ma, T-K.; Elliott, D. C.; Reid, S.; White, A. J. P.; Parsons, P. J.; Barrett, A. G. M. Meroterpenoid synthesis via sequential polyketide aromatization and cationic polyene cyclization: Total syntheses of (+)-hongoquercin $\mathrm{A}$ and B and related meroterpenoids. J. Org. Chem. 2018, 83, 13276-13286. 
7 Cano, M. J.; Bouanou, H.; Tapia, R.; Alvarez, E.; Alvarez-Manzaneda, R.; Chahboun, R.; Alvarez-Manzaneda, E. NIS- $\mathrm{PPh}_{3}$ : A selective reagent for the spiroannulation of $o$-allyl phenols. Total synthesis of corallidictyal D. J. Org. Chem. 2013, 78, 9196-9204.

8 Nieman, J. A.; Nair, S. K.; Heasley, S. E.; Schultz, B. L.; Zerth, H. M.; Nugent, R. A.; Chen, K.; Stephanski, K. J.; Hopkins, T. A.; Knechtel, M. L.; Oien, N. L.; Wieber, J. L.; Wathen, M. W. Modifications of C-2 on the pyrroloquinoline template aimed at the development of potent herpesvirus antivirals with improved aqueous solubility. Bioorganic \& Medicinal Chemistry Letters 2010, 20, 3039-3042.

9 Gupta, S.; Lin, Y.; Xia, Y.; Winka, D. J.; Lee, D. Alder-ene reactions driven by high steric strain and bond angle distortion to form benzocyclobutenes. Chem. Sci. 2019, 10, 2212-2217.

10 Shinozuka, T.; Yamamoto, Y.; Hasegawa, T.; Saito, K.; Naito, S. First total synthesis of sterenins A, C and D. Tetrahedron Lett. 2008, 49, 1619-1622.

11 Xie, L.; Takeuchi, Y.; Cosentino, L. M.; McPhail, A. T.; Lee, K. H. Anti-AIDS agents. 42. Synthesis and anti-HIV activity of disubstituted (3'R,4'R)-3',4'-di-O-(S)-camphanoyl-(+)-cis-khellactone analogues. J. Med. Chem. 2001, 44, 664-671.

12 Kim, K. H.; Noh, H. J.; Choi, S. U.; Lee, K. R. Isohericenone, a new cytotoxic isoindolinone alkaloid from Hericium erinaceum. J. Antibiot. 2012, 65, 575-577.

13 Yaoita, Y.; Danbara, K.; Kikuchi, M. Two new aromatic compounds from Hericium erinaceum (Bull.: Fr.) Pers. Chem. Pharm. Bull. 2005, 53, 1202-1203.

14 Ito-Kobayashi, M.; Aoyagi, A.; Tanaka, I.; Muramatsu, Y.; Umetani, M.; Takatsu, T. Sterenin A, B, C and D, novel 11b-hydroxysteroid dehydrogenase type 1 inhibitors from Stereum sp. SANK 21205. J. Antibiot. 2008, 61, 128135.

15 Frisch, M. J.; Trucks, G. W.; Schlegel, H. B.; Scuseria, G. E.; Robb, M. A.; Cheeseman, J. R.; Scalmani, G.; Barone, V.; Mennucci, B.; Petersson, G. A.; Nakatsuji, H.; Caricato, M.; Li, X.; Hratchian, H. P.; Izmaylov, A. F.; Bloino, J.; Zheng, G.; Sonnenberg, J. L.; Hada, M.; Ehara, M.; Toyota, K.; Fukuda, R.; Hasegawa, J.; Ishida, M.; Nakajima, T.; Honda, Y.; Kitao, O.; Nakai, H.; Vreven, T.; Montgomery, J. A.; Peralta, Jr.; J. E.; Ogliaro, F.; Bearpark, M.; Heyd, J. J.; Brothers, E.; Kudin, K. N.; Staroverov, V. N.; Keith, T.; Kobayashi, R.; Normand, J.; Raghavachari, K.; Rendell, A.; Burant, J. C.; Iyengar, S. S.; Tomasi, J.; Cossi, M.; Rega, N.; Millam, J. M.; Klene, M.; Knox, J. E.; Cross, J. B.; Bakken, V.; Adamo, C.; Jaramillo, J.; Gomperts, R.; Stratmann, R. E.; Yazyev, O.; Austin, A. J.; Cammi, R.; Pomelli, C.; Ochterski, J. W.; Martin, R. L.; Morokuma, K.; Zakrzewski, V. G.; Voth, G. A.; Salvador, P.; Dannenberg, J. J.; Dapprich, S.; Daniels, A. D.; Farkas, O.; Foresman, J. B.; Ortiz, J. V.; Cioslowski, J.; Fox, D. J. Gaussian 09, Revision C.01, Gaussian Inc.: Wallingford, CT (2010).

16 Zhao, Y.; Truhlar, D. G. The M06 suite of density functionals for main group thermochemistry, thermochemical kinetics, noncovalent interactions, excited states, and transition elements: Two new functionals and systematic testing of four M06-class functionals and 12 other functionals. Theor. Chem. Acc. 2008, 120, 215-241.

17 Marenich, A. V.; Cramer, C. J.; Truhlar, D. G. Universal solvation model based on solute electron density and on a continuum model of the solvent defined by the bulk dielectric constant and atomic surface tensions. J. Phys. Chem. B, 2009, 113, 6378-6396.

\section{Copies of ${ }^{1} \mathrm{H}$ and ${ }^{13} \mathrm{C}$ NMR spectra}




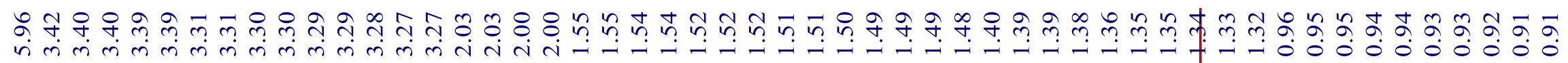

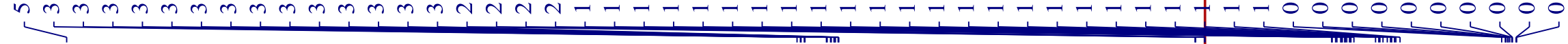

\section{$\mathrm{S} 1 / \mathrm{H}$}
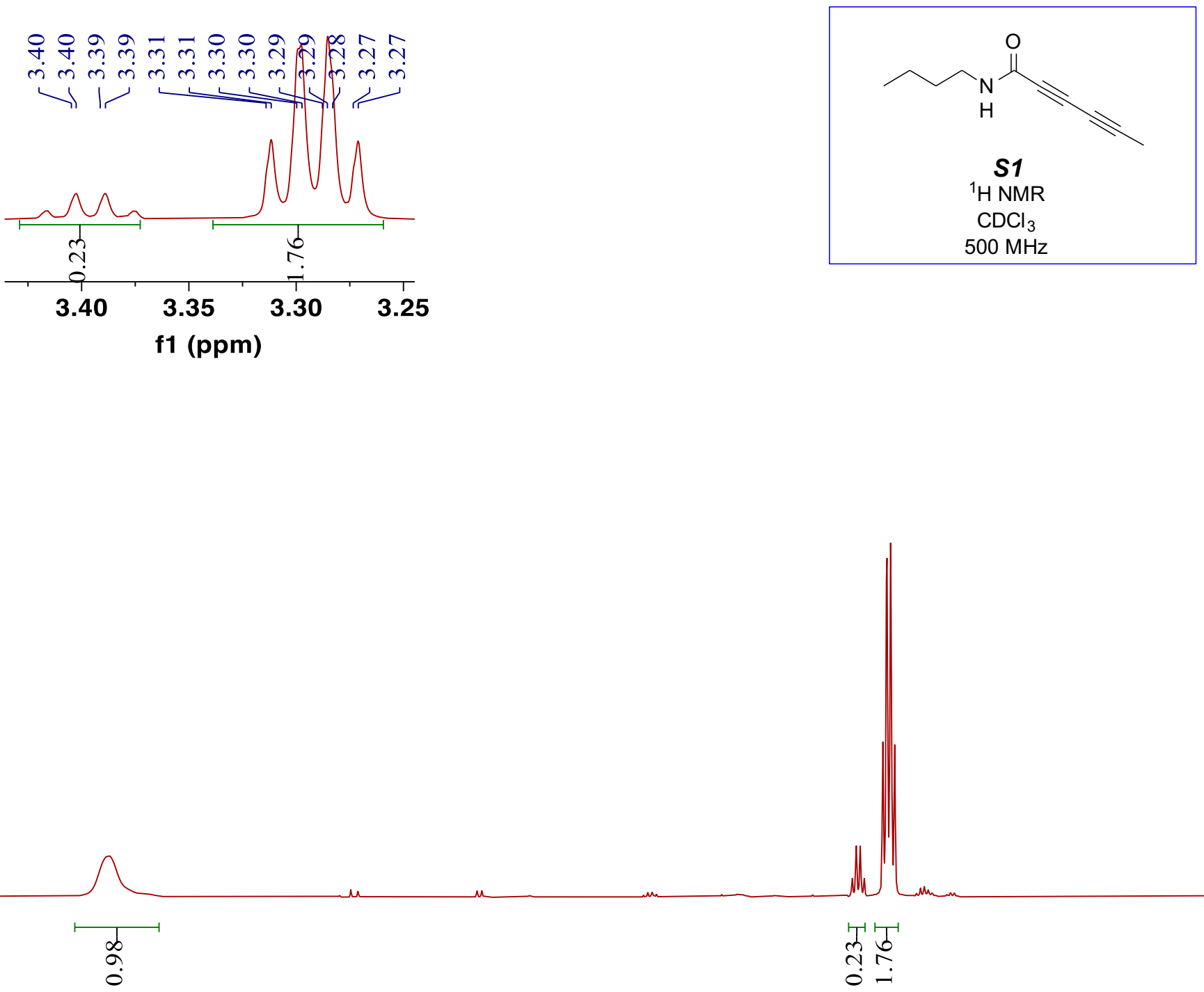

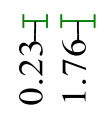

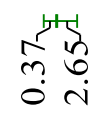

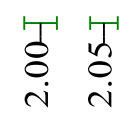

它 

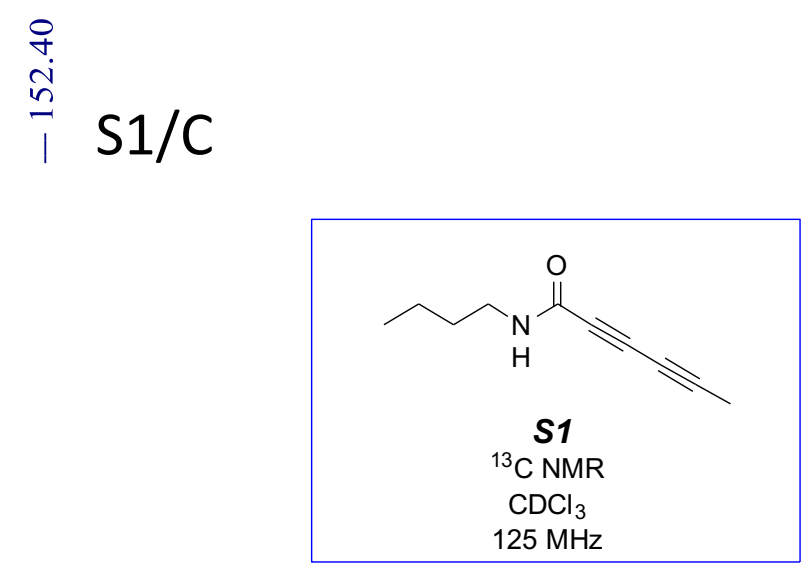

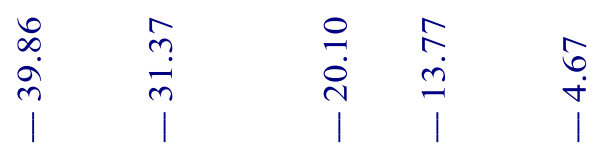
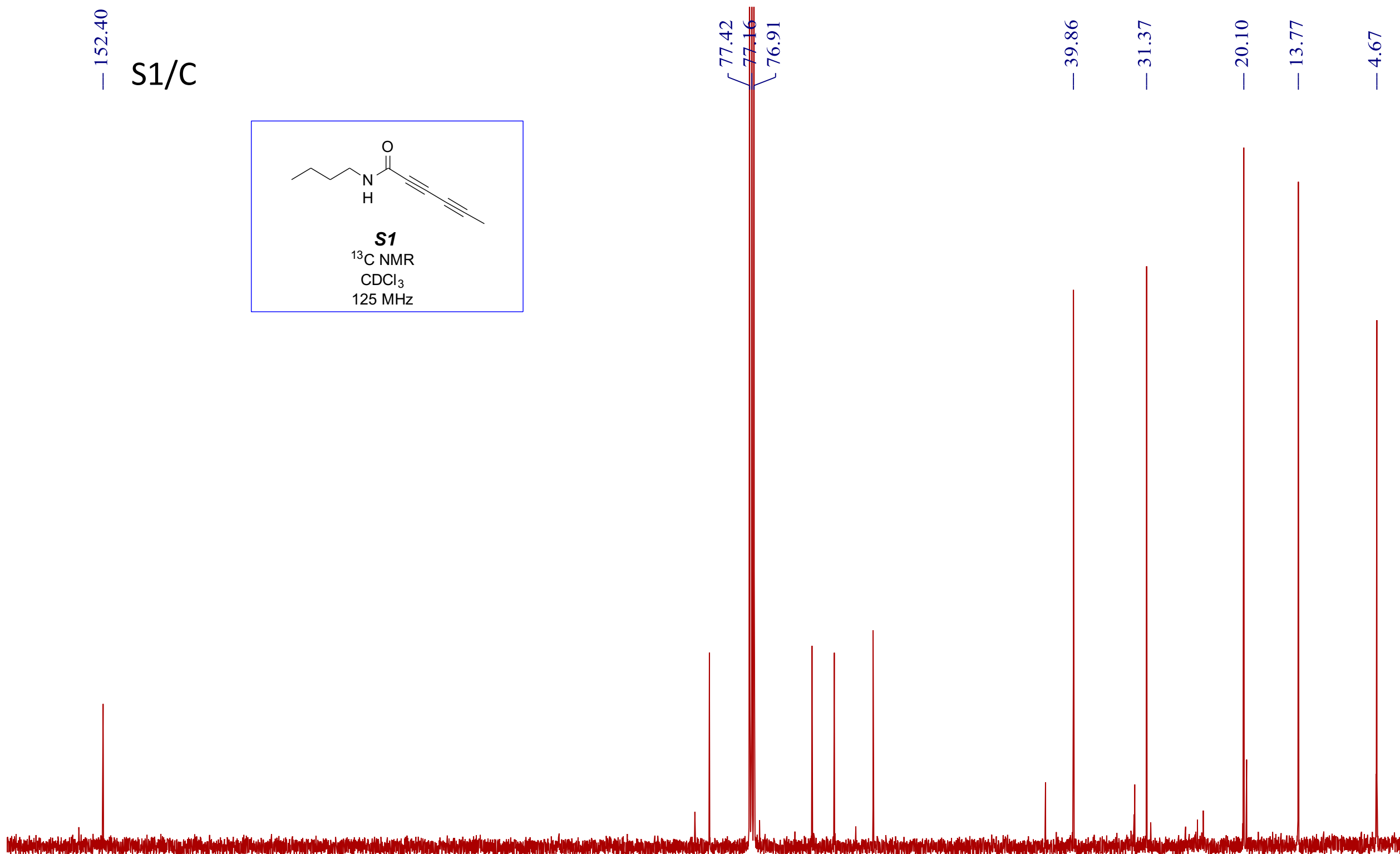
mir

$10 \mathrm{a} / \mathrm{H}$

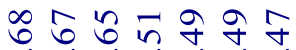

$\dot{m} m m \dot{m} m$

$\rightarrow 1$

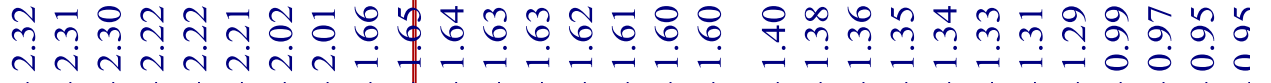
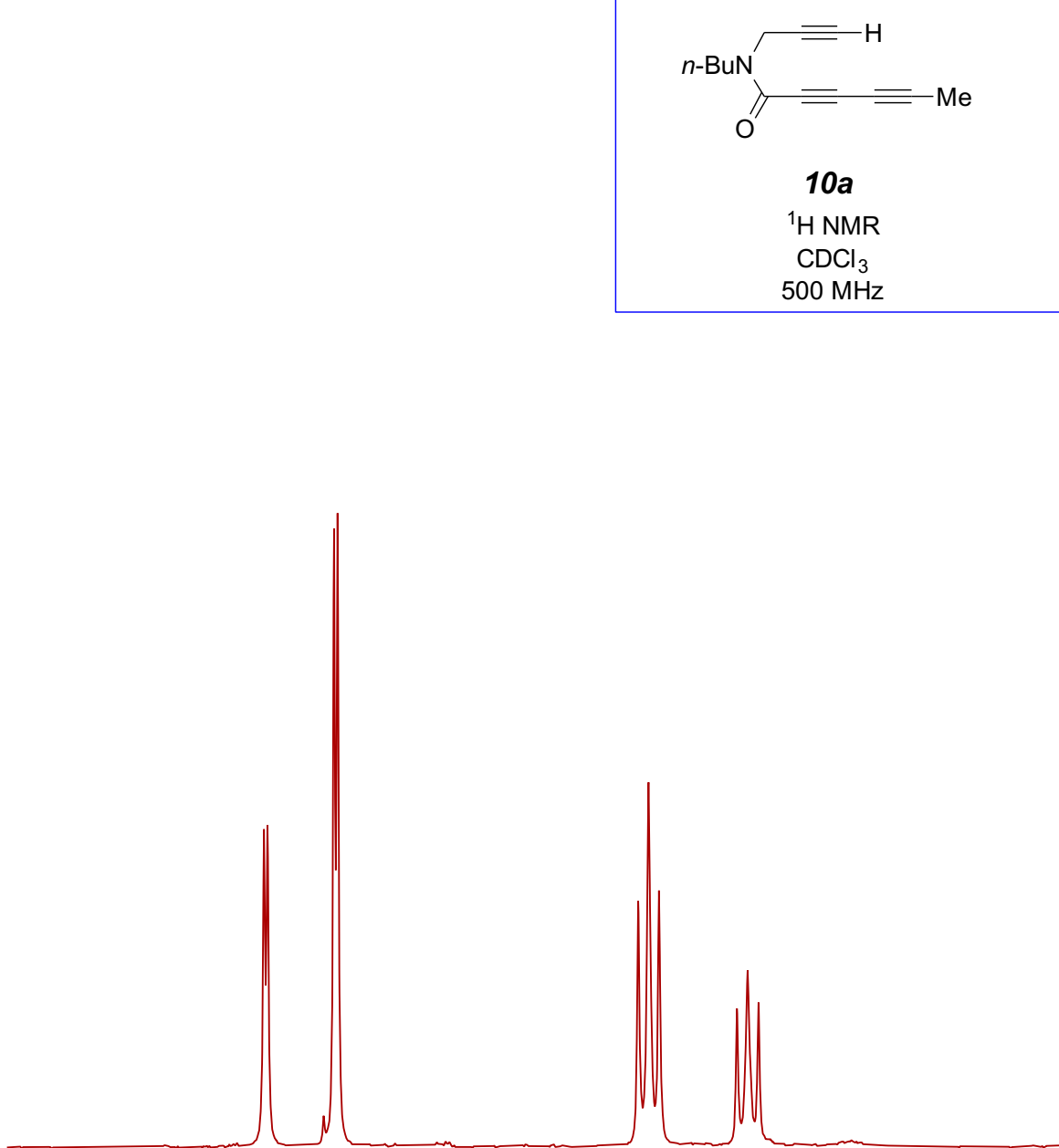

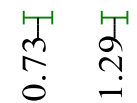

$\stackrel{n}{n} \stackrel{n}{n} \stackrel{0}{0}$

$\begin{array}{cc}\uparrow & 1 \\ \text { ñ } & \infty \\ 0 & 0\end{array}$

官
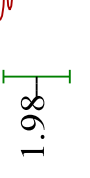

2.9 f1 (ppm) 
S59 of S139

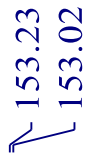

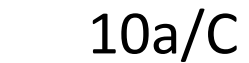

$\bar{\infty} \overrightarrow{0}$

लं

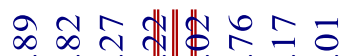

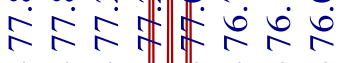

11

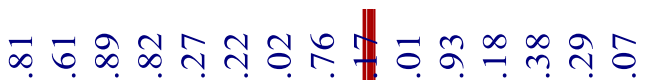

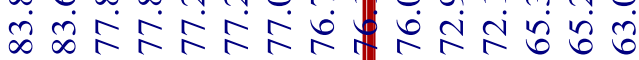

\& $n$

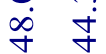

ㅇำ $\ln ^{2}$

m mं

ㄴำㄴำ

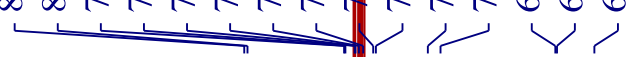

| 1

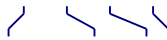

iㅇ

准

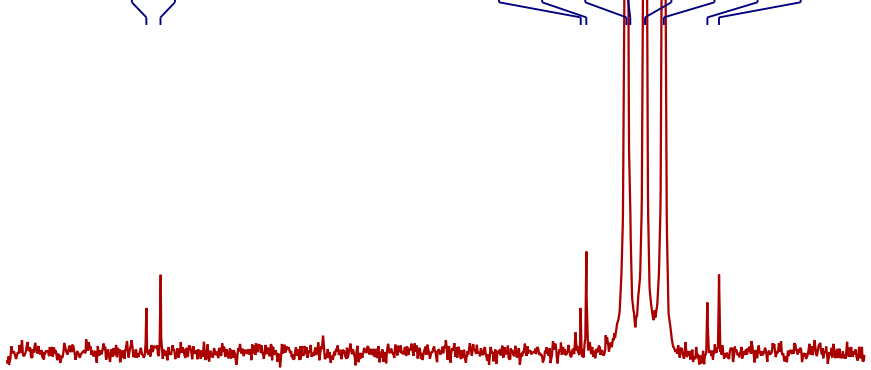

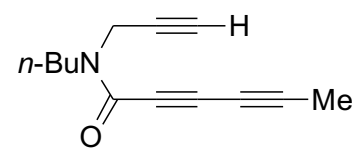

$10 a$

${ }^{13} \mathrm{C}$ NMR

$\mathrm{CDCl}_{3}$ $125 \mathrm{MHz}$

\section{|}

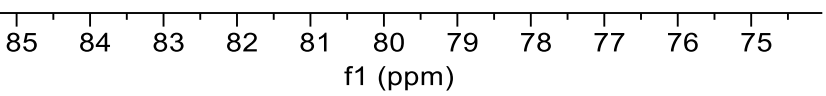

$\begin{array}{lllllllllllllllllllllllllllllll}55 & 150 & 145 & 140 & 135 & 130 & 125 & 120 & 115 & 110 & 105 & 100 & 95 & 90 & 85 & 80 & 75 & 70 & 65 & 60 & 55 & 50 & 45 & 40 & 35 & 30 & 25 & 20 & 15 & 10 & 5\end{array}$ 
Zhu, Zhang, \& Hoye

Supporting Information

$\mathrm{S} 60$ of $\mathrm{S} 139$

กิ ขุ

inj

$11 a / H$

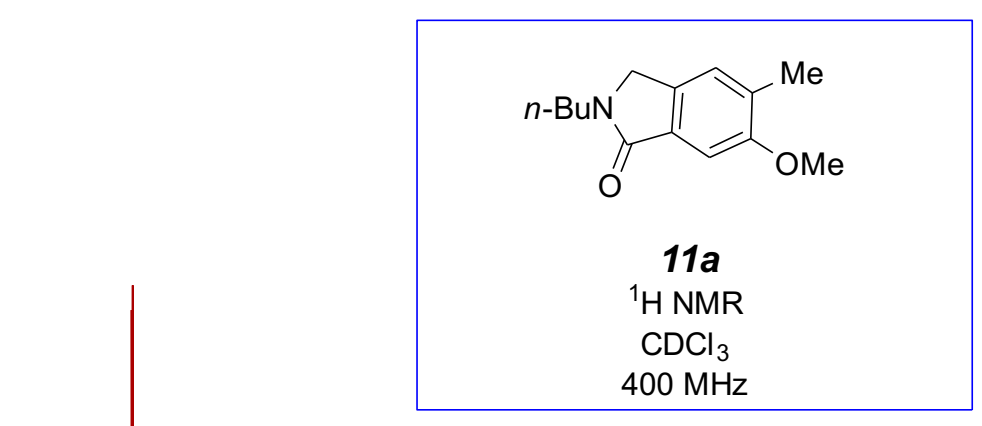

†

क రీ

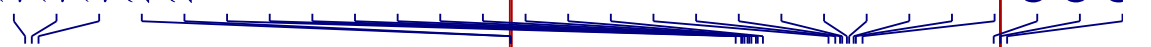

†

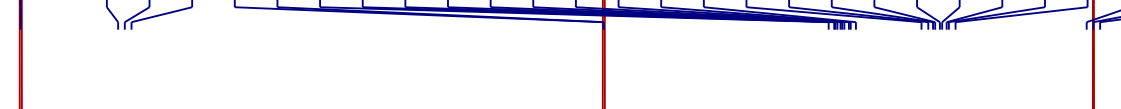

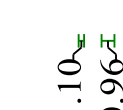

일

7.5

7.0

6.5

6.0

4.5

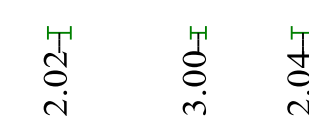

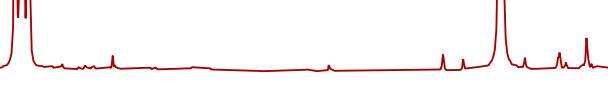

旿




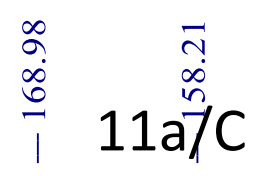

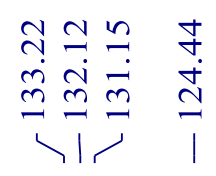

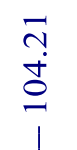

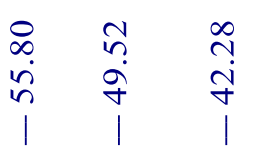

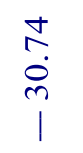

ำ ส้

ํㅗㅇ
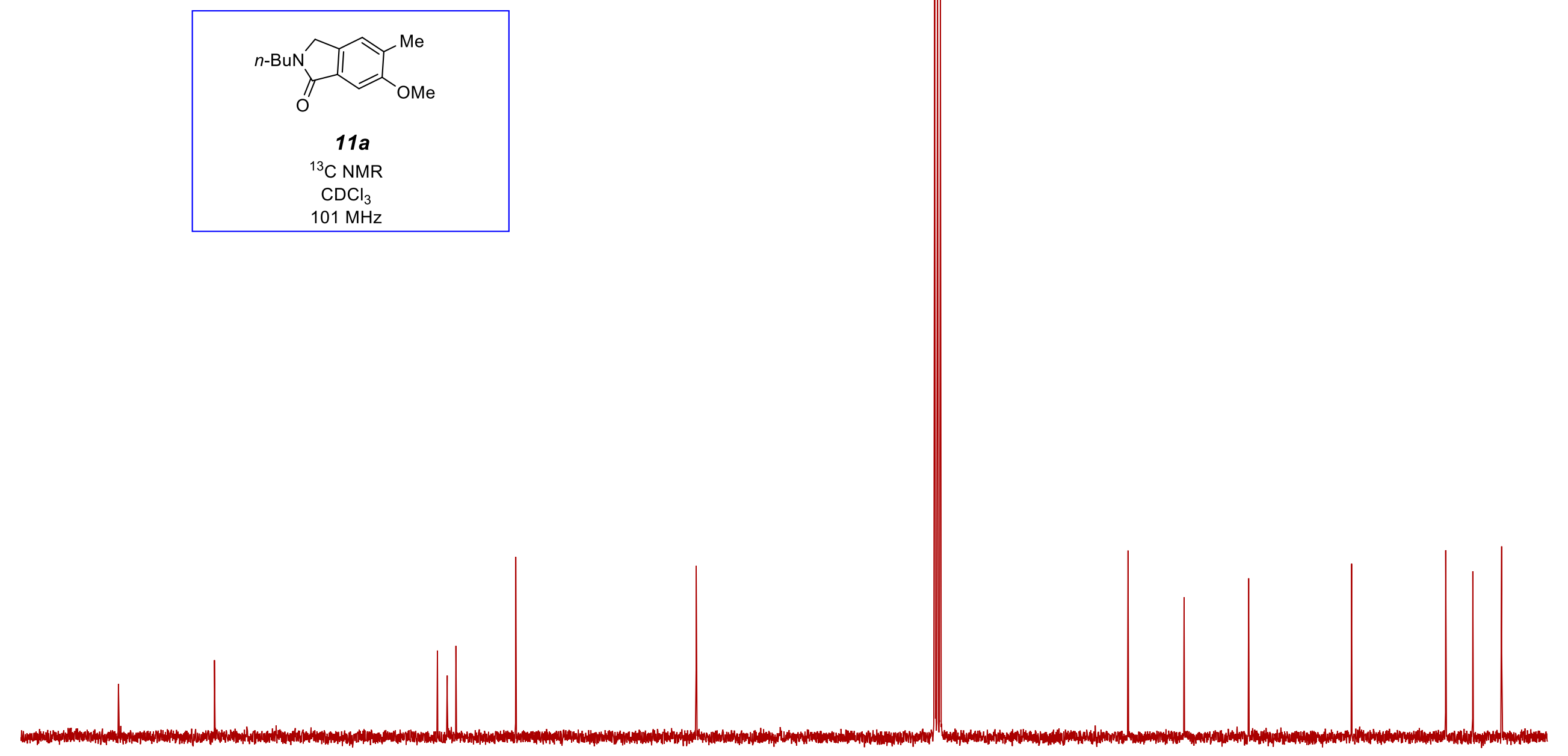


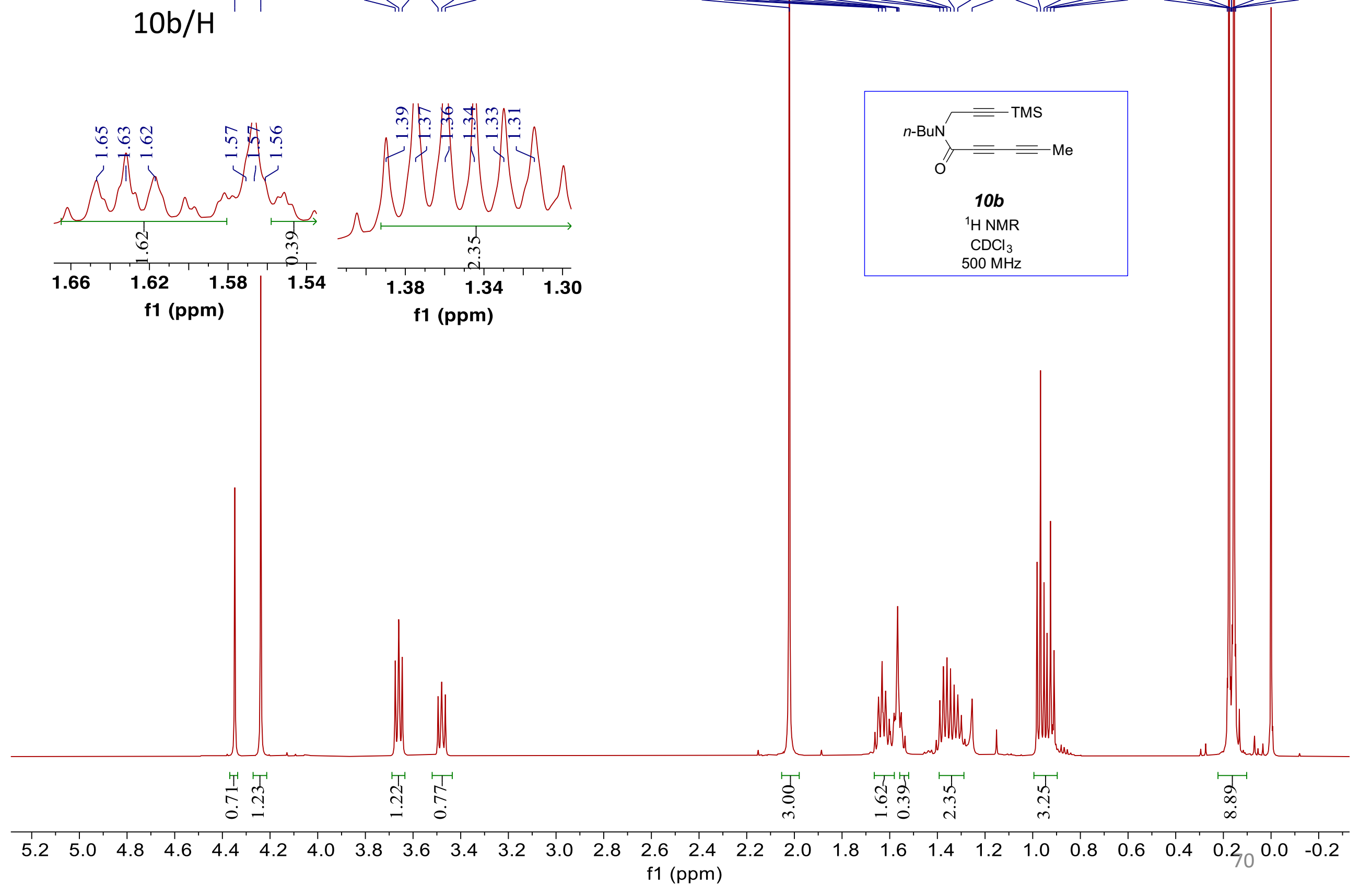

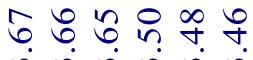

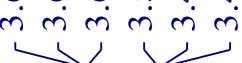
$10 \mathrm{~b} / \mathrm{H}$

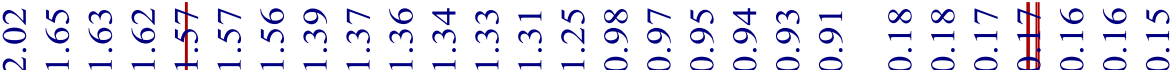

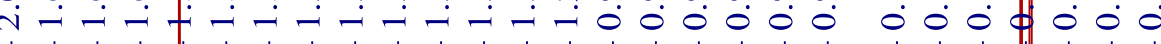

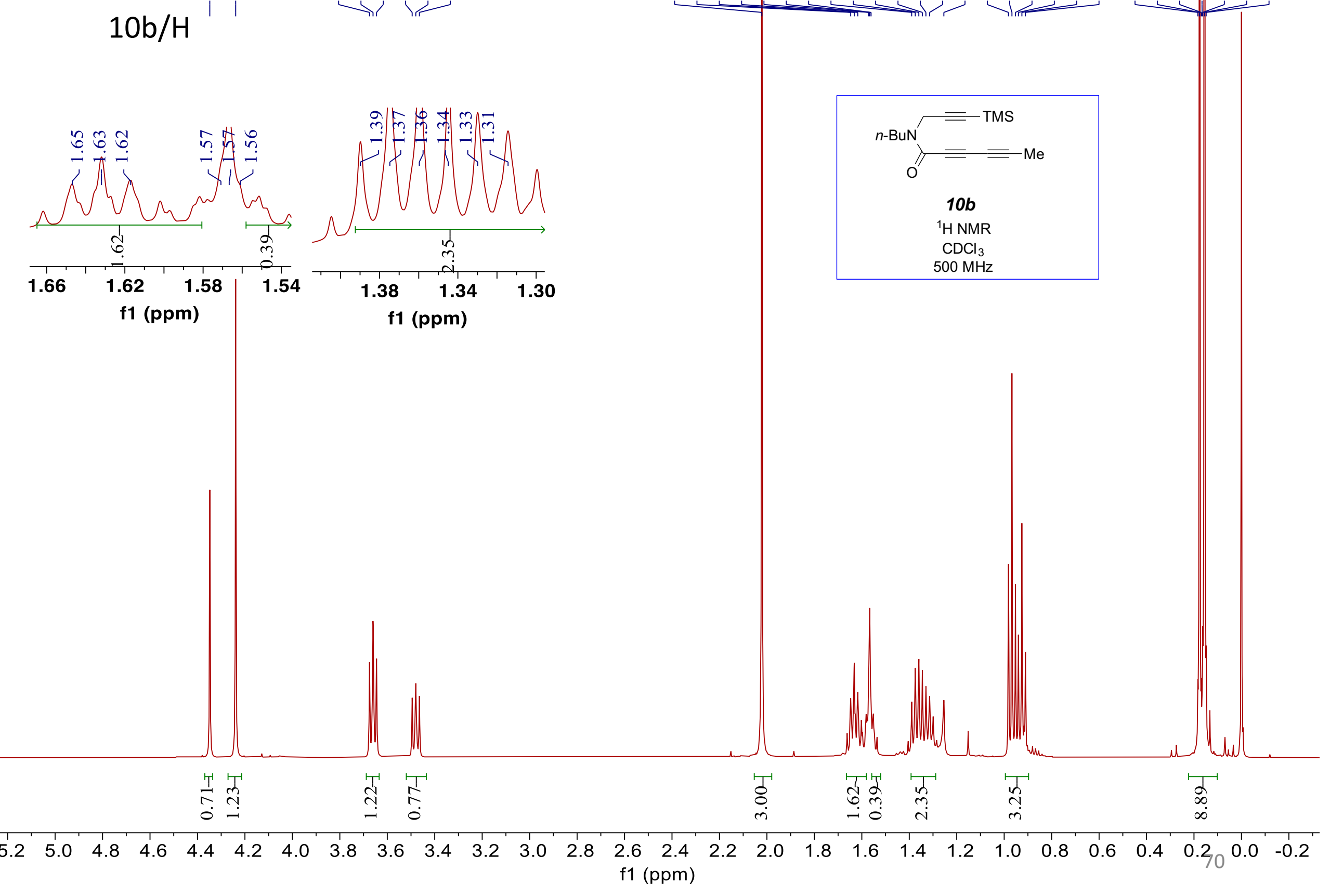




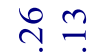

ñ

先 $10 \mathrm{~b} / \mathrm{C}$

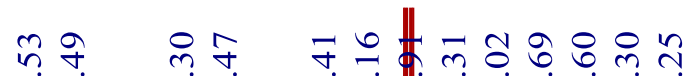

ำ

ลे ลे

$a \infty$

$\hat{\imath}$

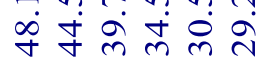

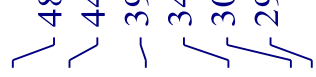

ปัँ オே

กั่

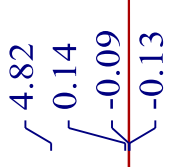




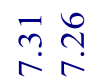

$11 \mathrm{~b} / \mathrm{H}$

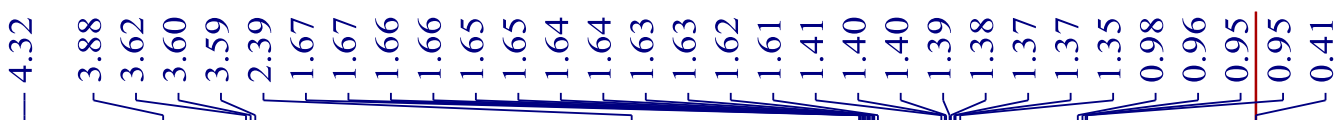

$11 b$
${ }^{1} \mathrm{NMR}_{3}$
$\mathrm{CDCl}_{3}$
$500 \mathrm{MHz}$

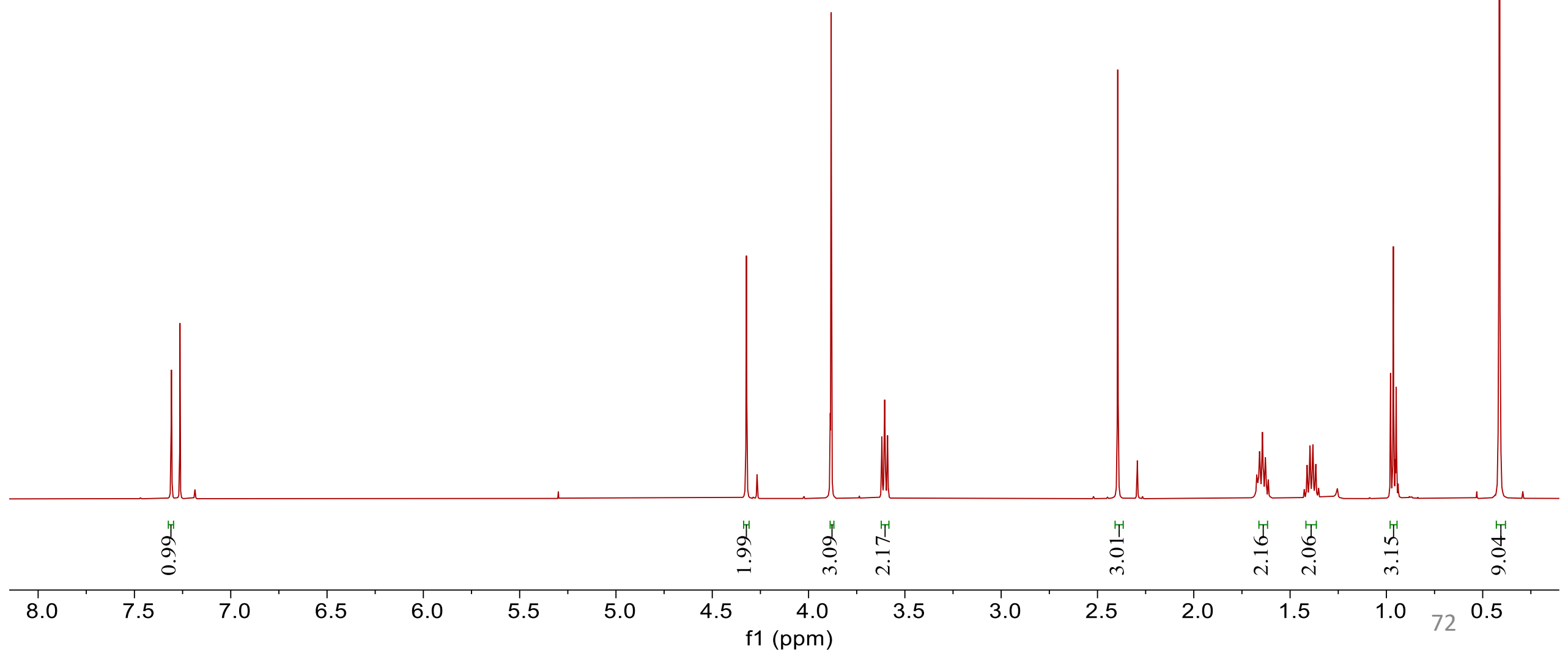




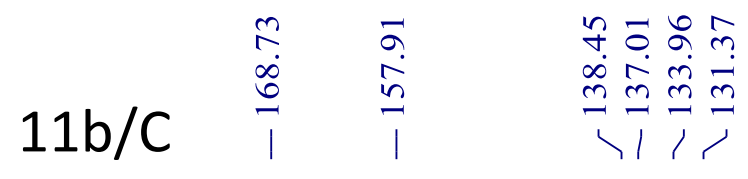

$\underset{\substack{n \\ \mathfrak{o} \\ 1}}{1}$

극

in i

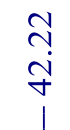

$\stackrel{\infty}{\infty}$

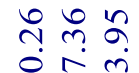

I I

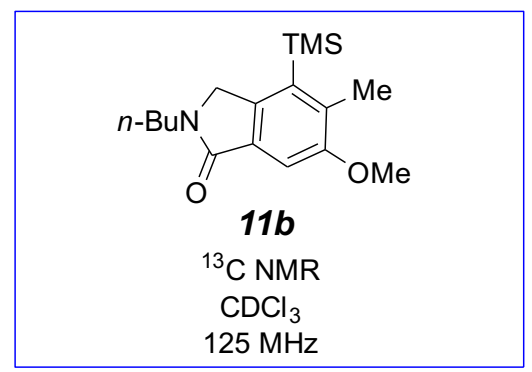

\begin{tabular}{lllllllllllllllllllllll}
\hline 10 & 200 & 190 & 180 & 170 & 160 & 150 & 140 & 130 & 120 & 110 & $\begin{array}{c}100 \\
\mathrm{f} 1(\mathrm{ppm})\end{array}$ & 90 & 80 & 70 & 60 & 50 & 40 & 30 & 20 & 10 & 0 & -
\end{tabular}




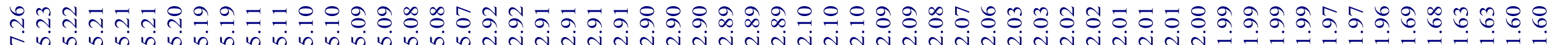
$15 / \mathrm{H}$

तิ तิ

in
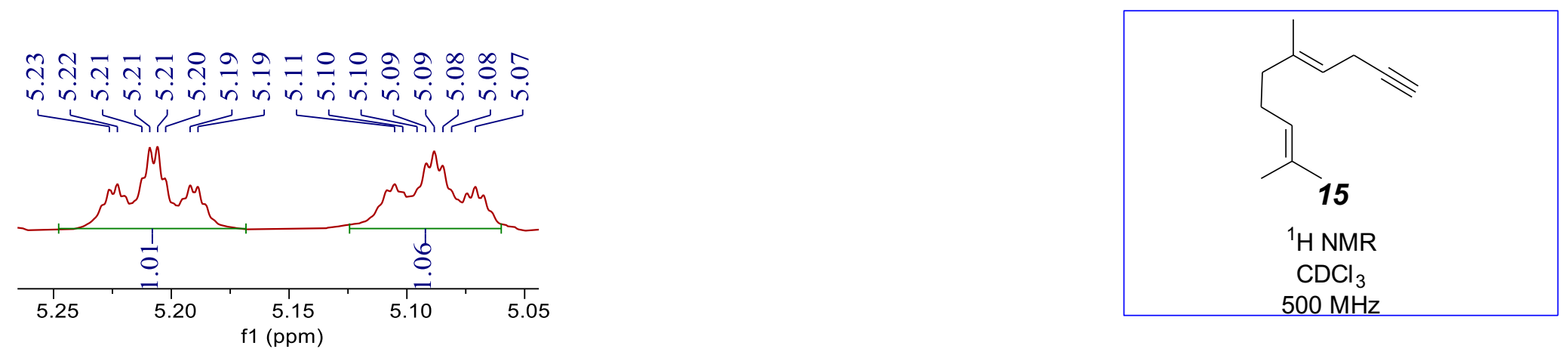

IIIII

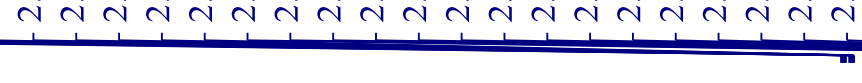




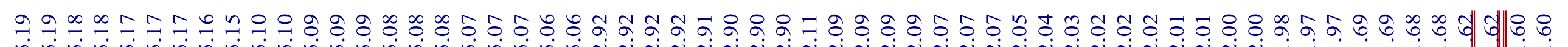

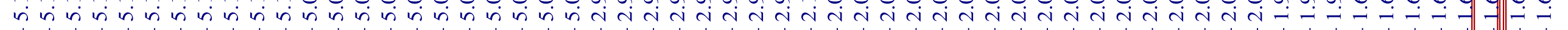
$\mathrm{S} 2 \mathrm{H}$

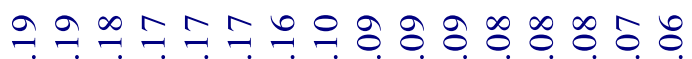
in in in
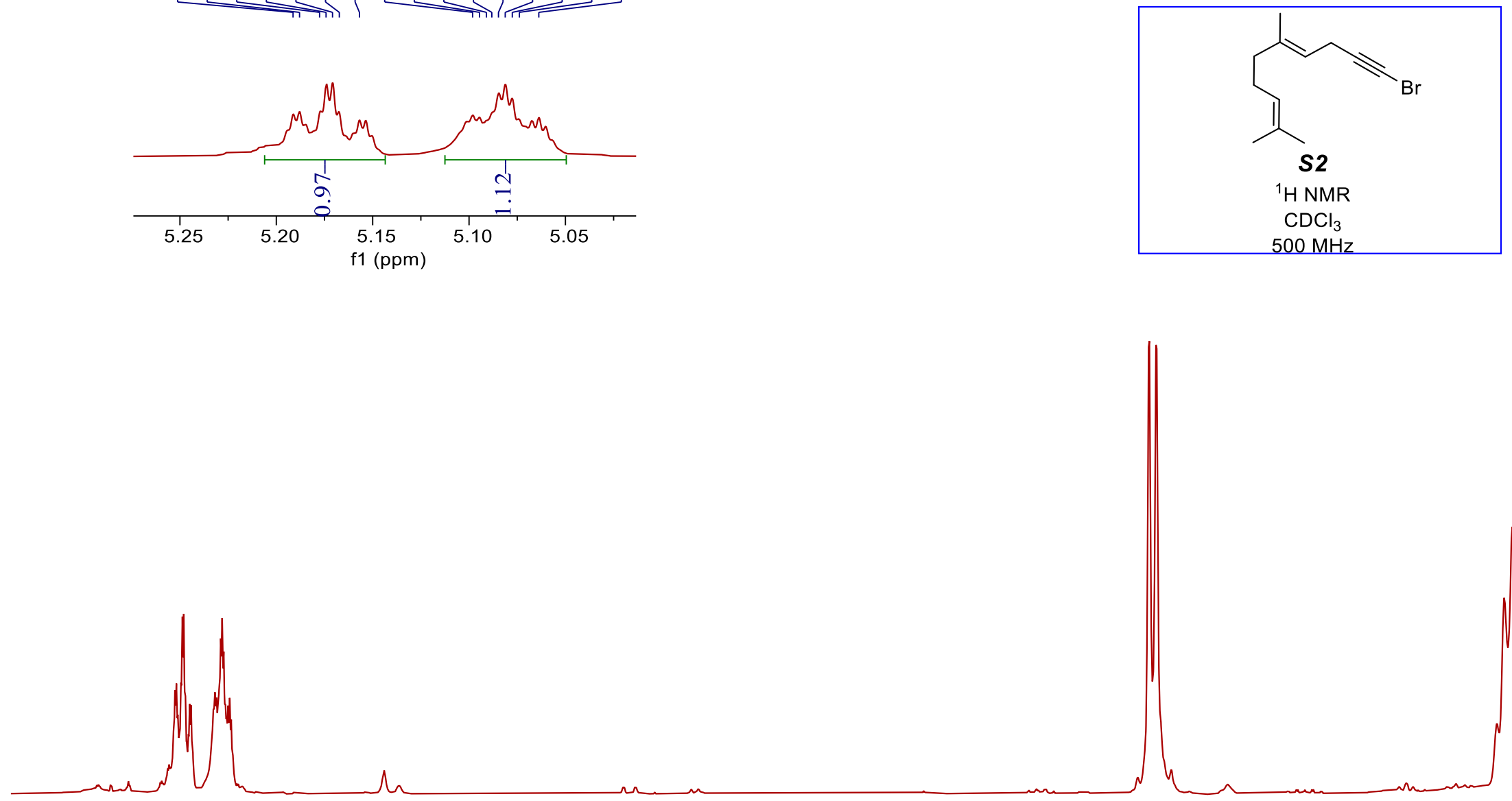

ํํำ
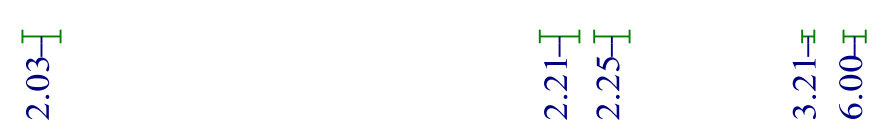

(1,

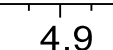

4.7

$4.5 \quad 4.3$

4.1

3.9

3.7

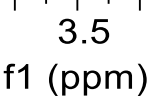

$\begin{array}{lllll}3.3 & 3.1 & 2.9 & 2.7\end{array}$

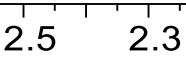

2.1

$1.9761 .7 \quad 1.5$ 

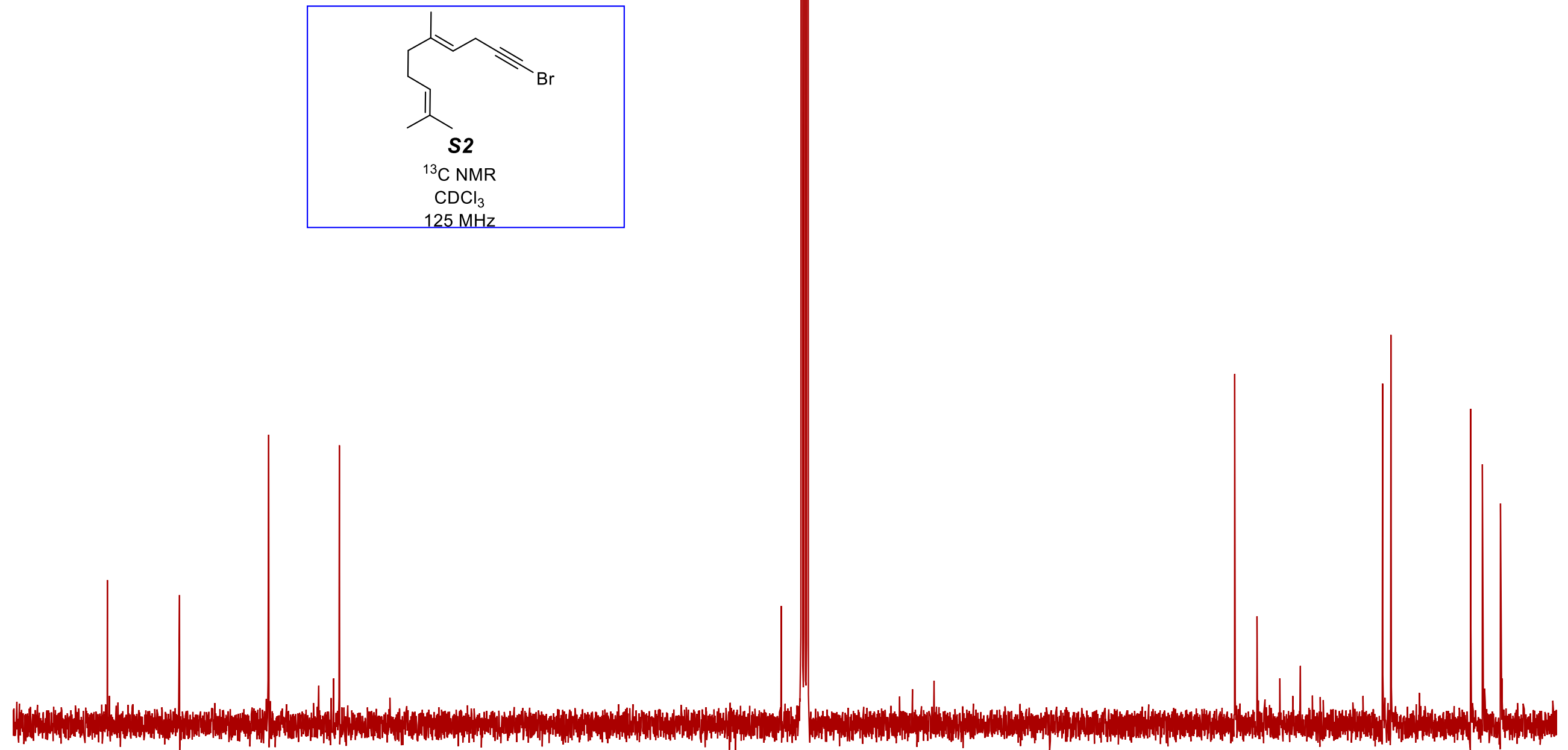

$$
145
$$$$
130125
$$$$
120
$$$$
115
$$$$
\begin{array}{lll}
110 & 105 & 100
\end{array}
$$ 


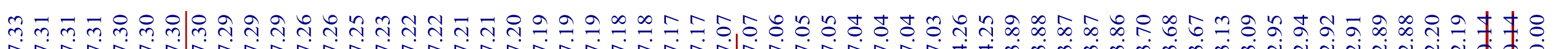

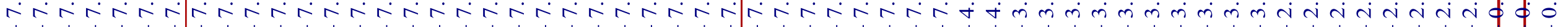

S3/1
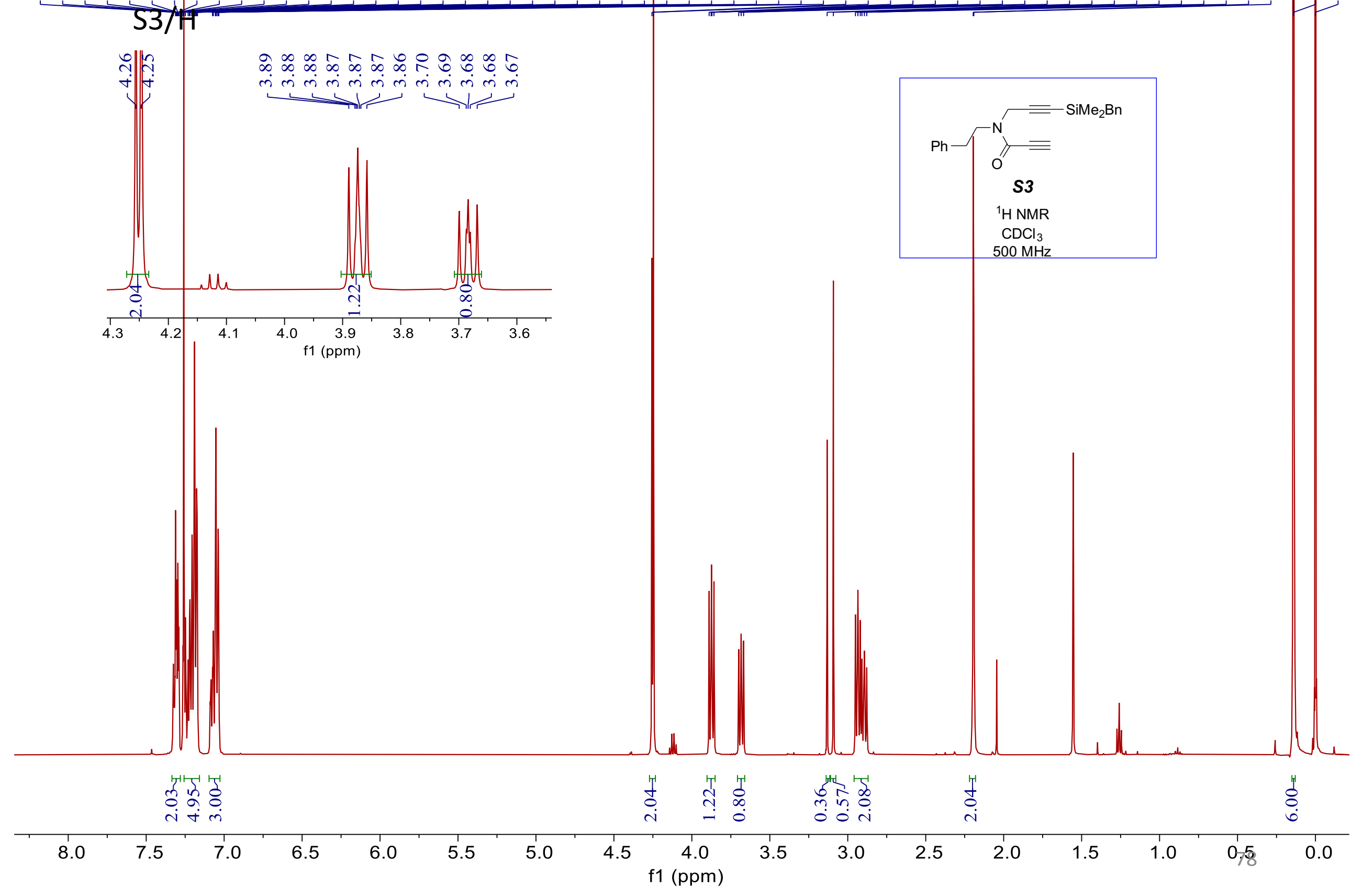


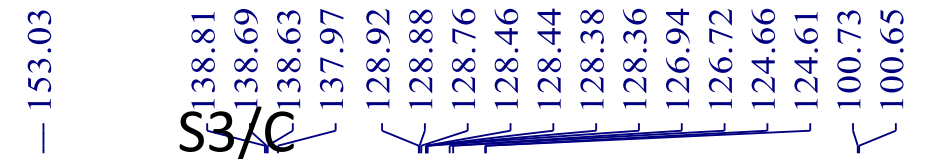

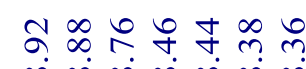

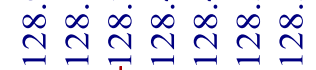

$\pi)$

$\stackrel{\dot{0}}{\stackrel{0}{N}}$

으.

iा

İ

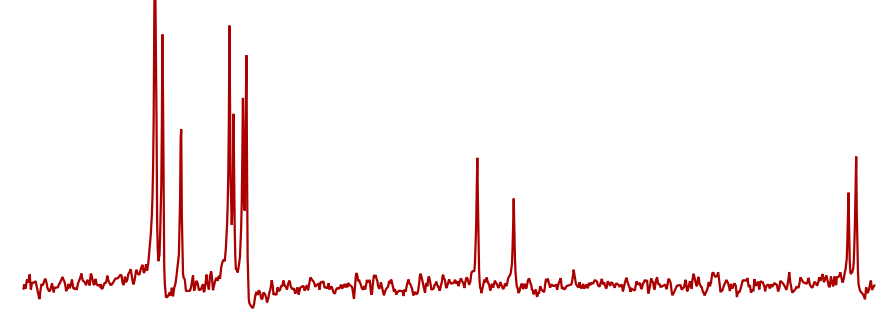

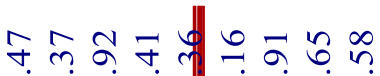

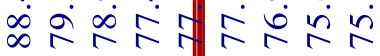

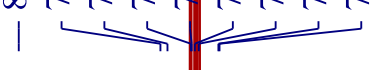

1

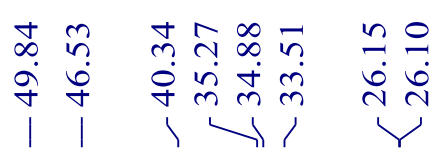

능

पm $\mathrm{m}$ त

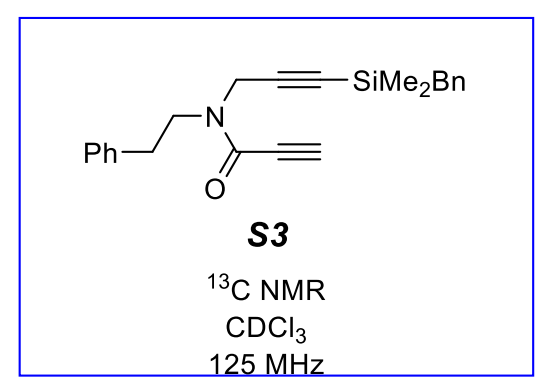

$125 \mathrm{MHz}$
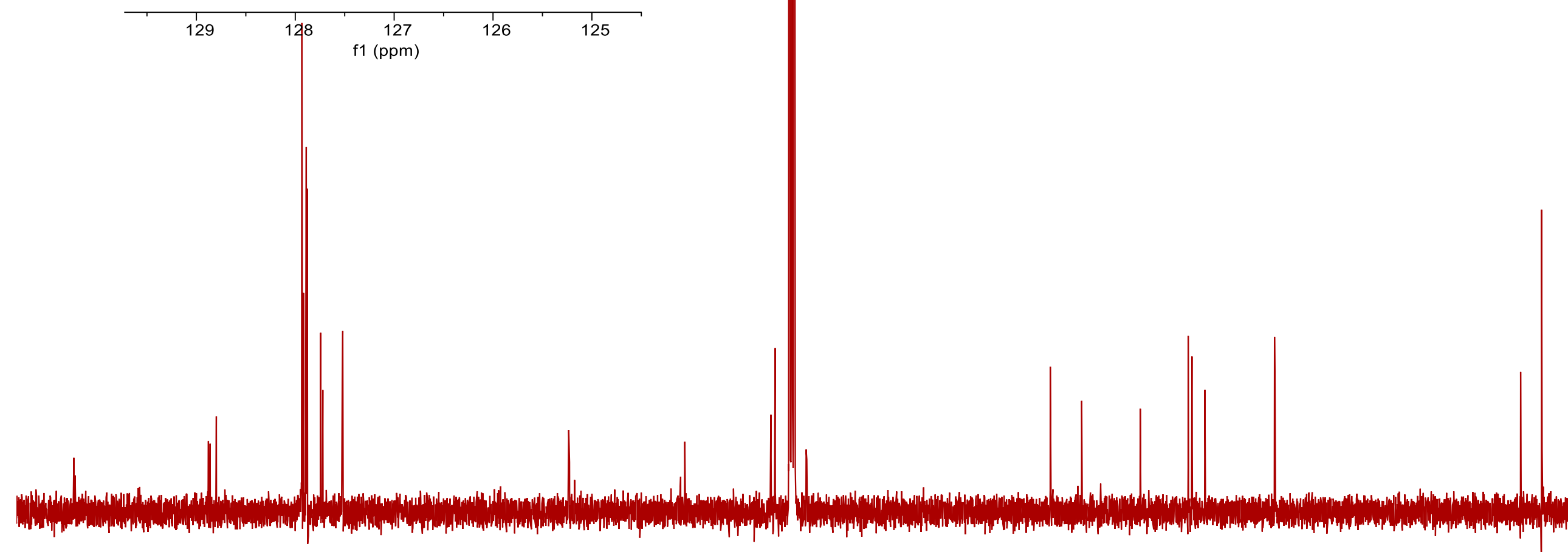

70

60

50

40

30

20

109




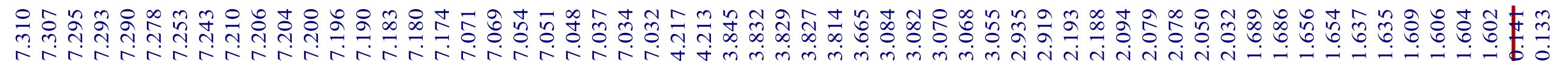
S4/H
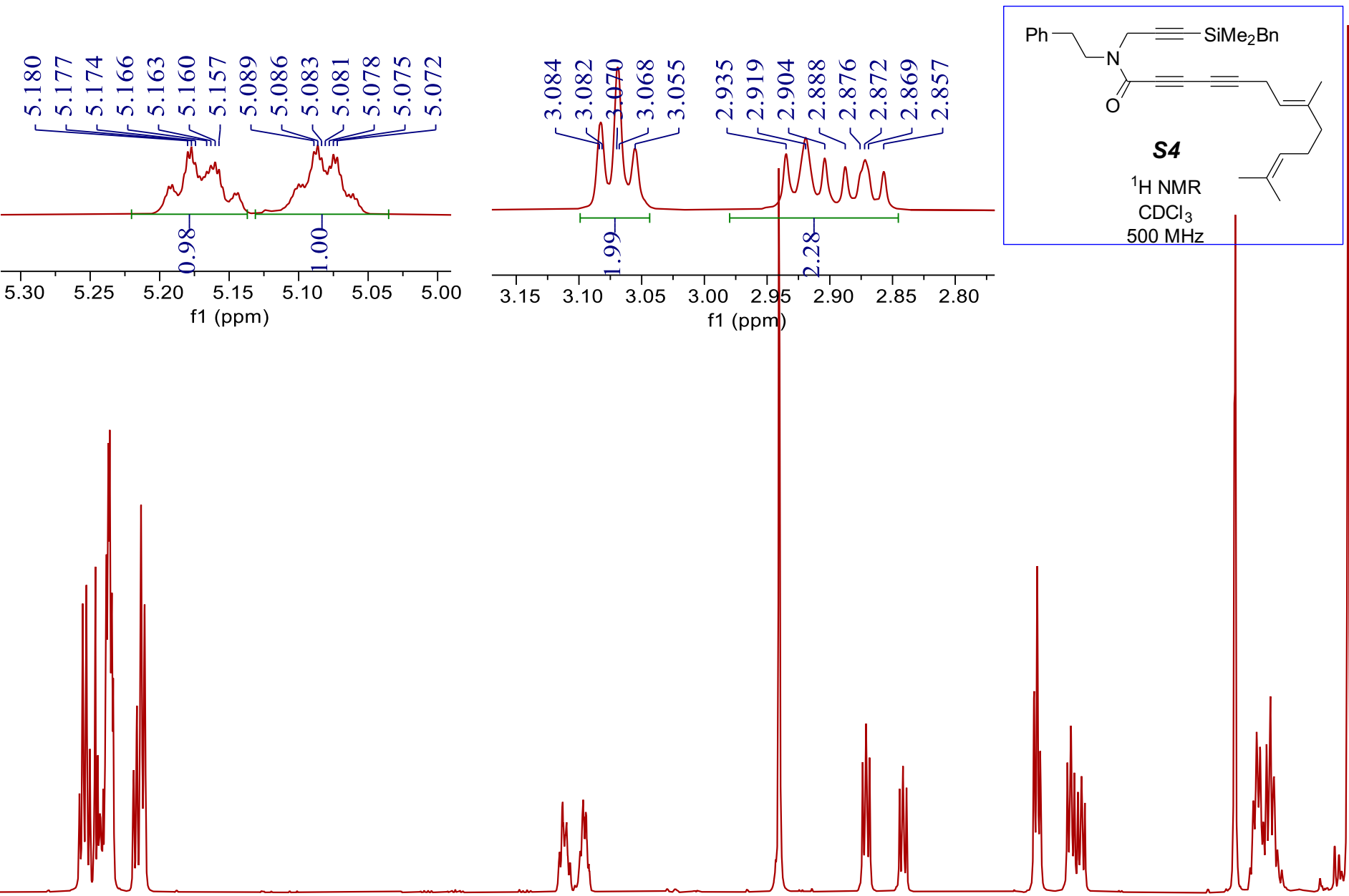

\section{客空军}

它'

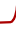

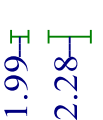

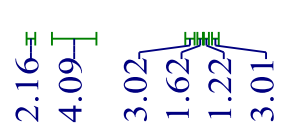

$7.5 \quad 7.0$

6.5

$6.0 \quad 5.5$

5.0

4.5

4.0

3.5

1

2.5

2.0

f1 (ppm) 
ते

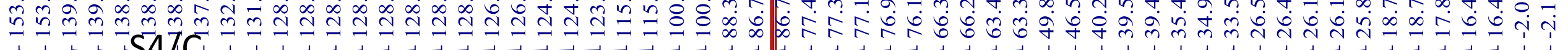
r
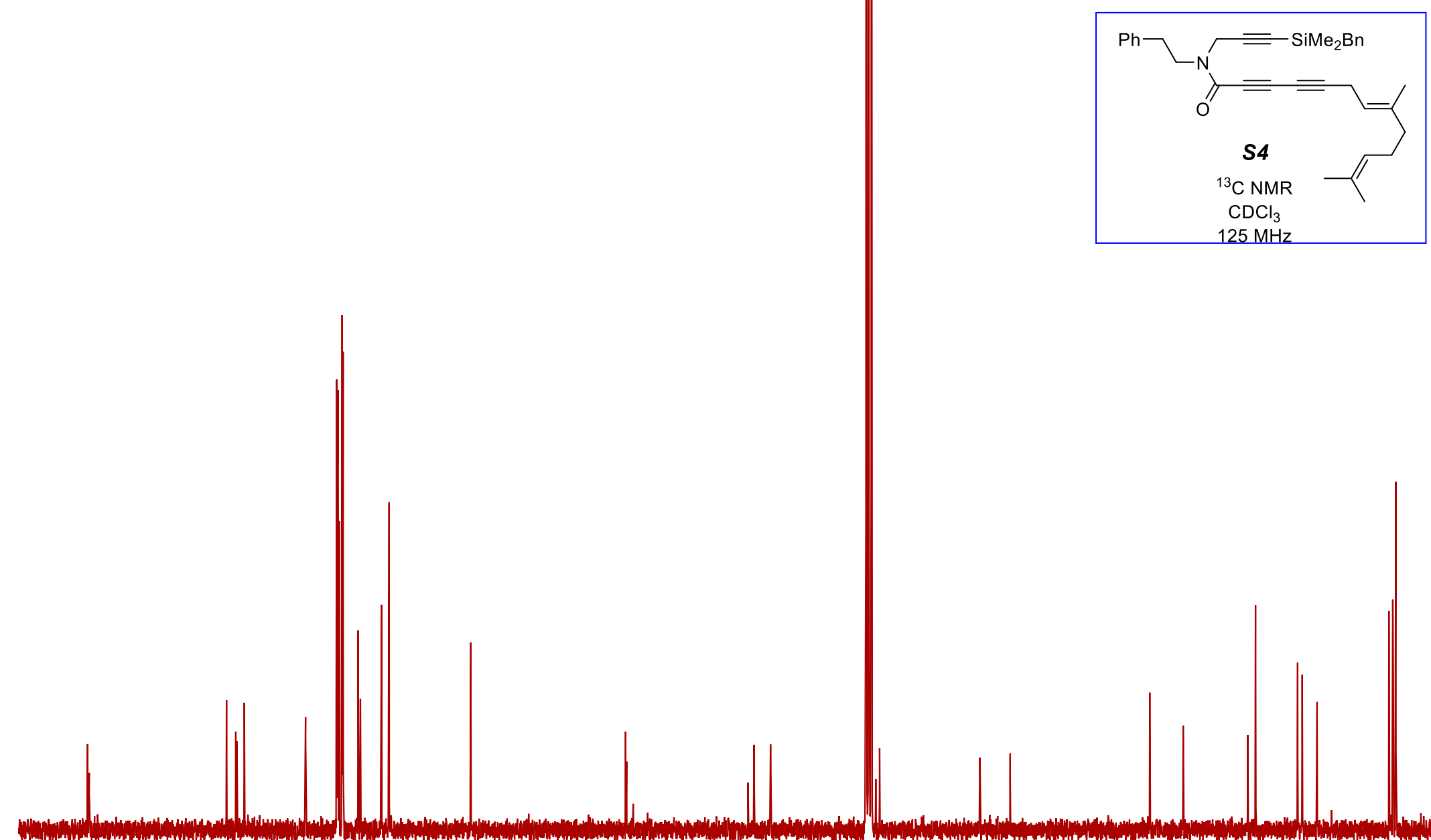


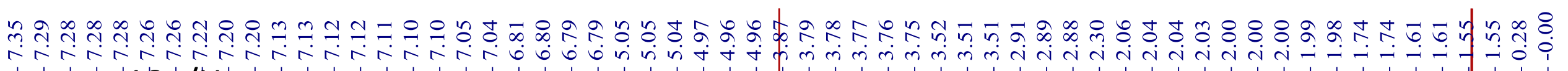

1001
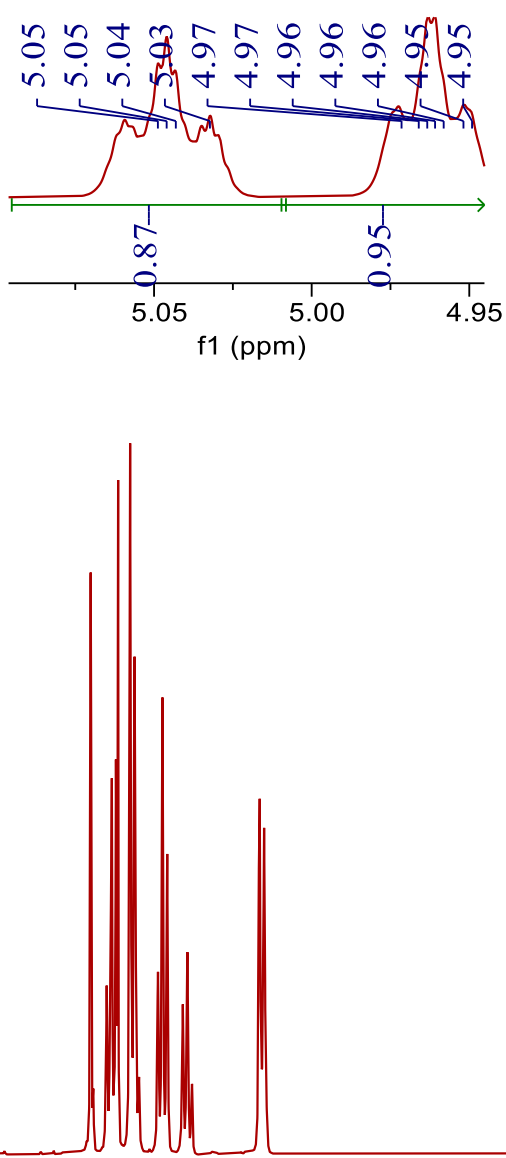

की
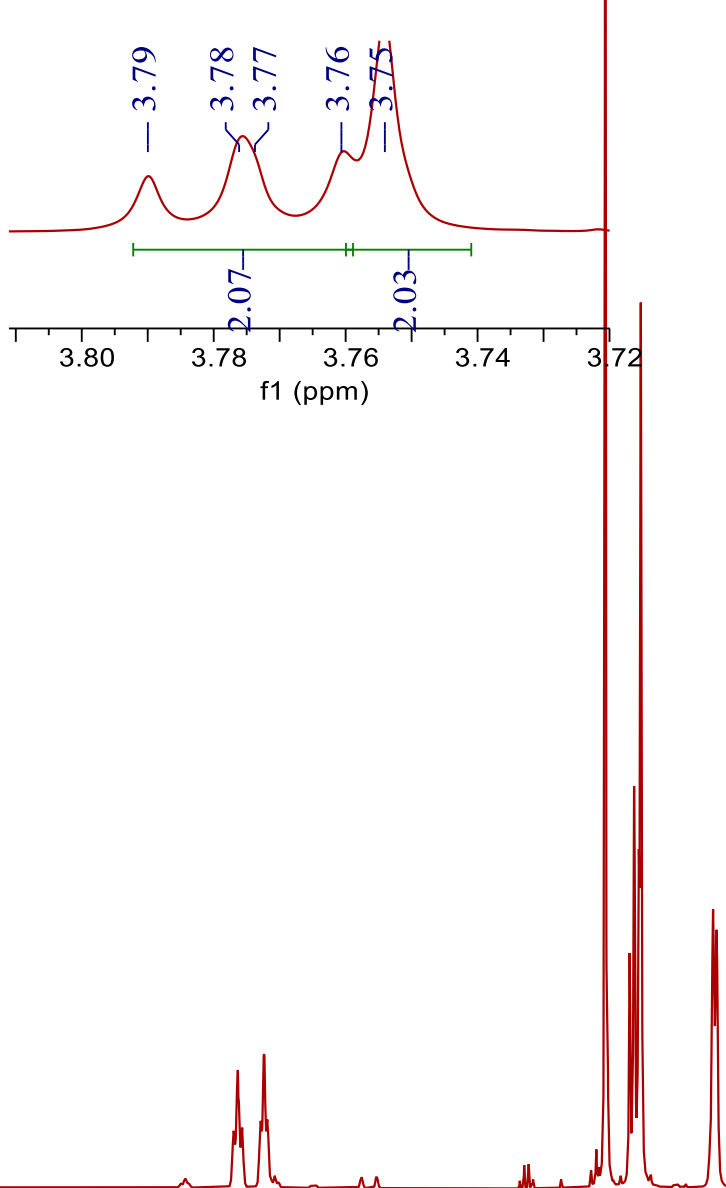

๙ึ.

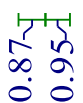

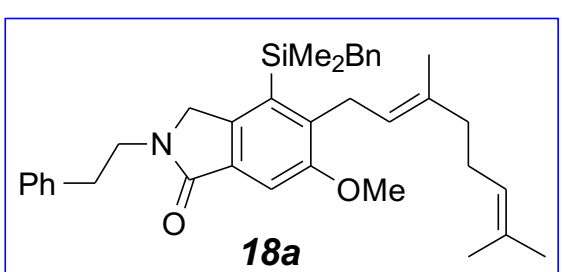

${ }^{1} \mathrm{H}$ NMR

$\mathrm{CDCl}_{3}$

$500 \mathrm{MHz}$

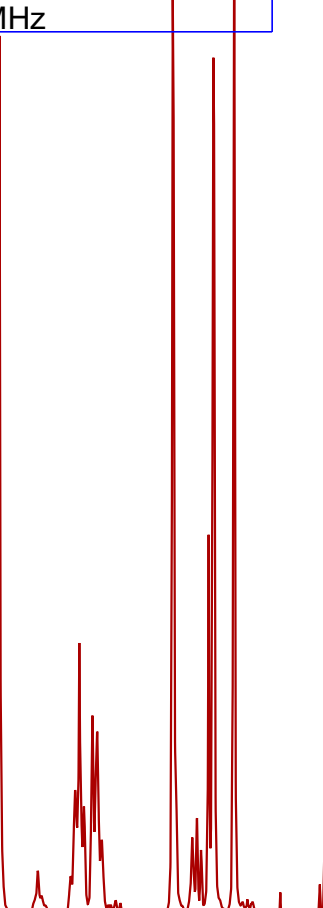

ํ.

$\begin{array}{lllll}7.5 & 7.0 & 6.5 & 6.0 & 5.5\end{array}$

$5.0 \quad 4.5$

2.0

1.5

0,52

0.0 


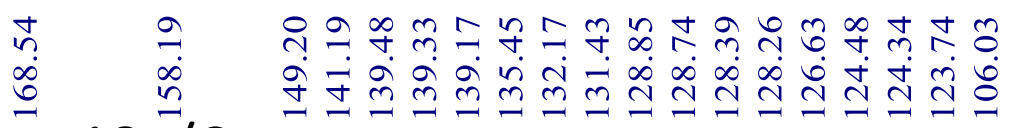
$18 \mathrm{a} / \mathrm{C}$

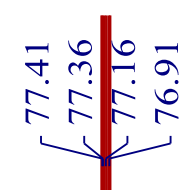

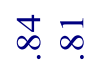

in in

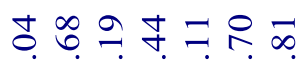
Ұं कें

$\infty$

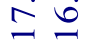

$1>$

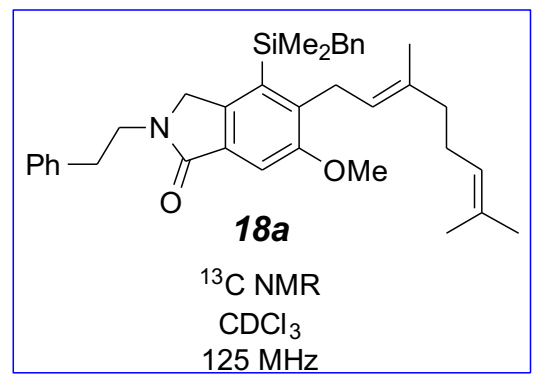

\begin{tabular}{|c|c|c|c|c|c|c|c|c|c|c|c|c|c|c|c|c|c|c|}
\hline 180 & 170 & 160 & 150 & 140 & 130 & 120 & 110 & 100 & $\begin{array}{r}90 \\
\mathrm{f} 1(\mathrm{ppn}\end{array}$ & 80 & 70 & 60 & 50 & 40 & 30 & 20 & $83^{10}$ & 0 \\
\hline
\end{tabular}




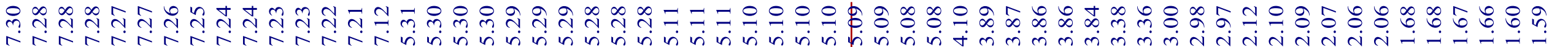

\section{9/H}

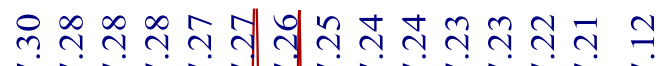
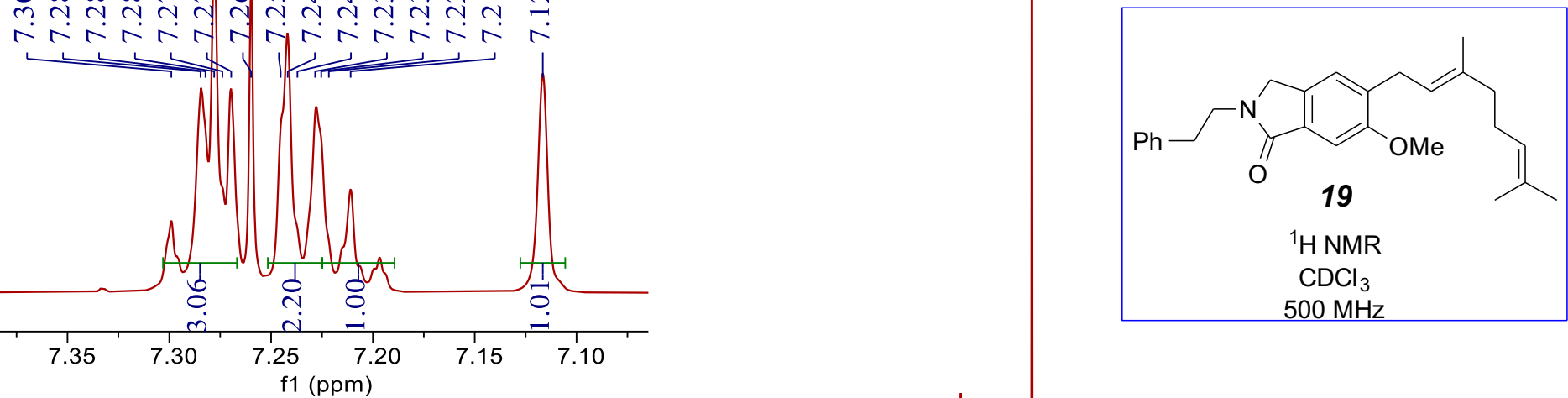

f1 (ppm)

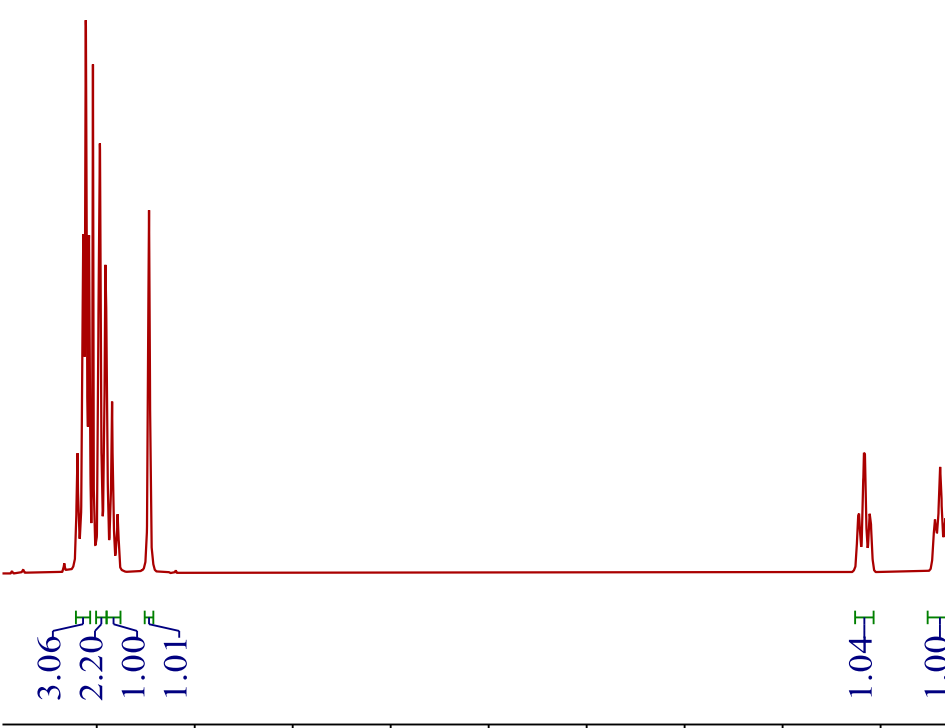

7.0

6.5

6.0

5.5

5.0

f1 (ppm)

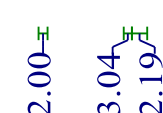

4.0

3.5

3.5

5


$\diamond \vec{\sim} \infty \underset{\infty}{\infty}$

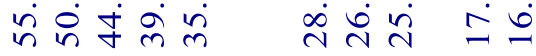

ஸి
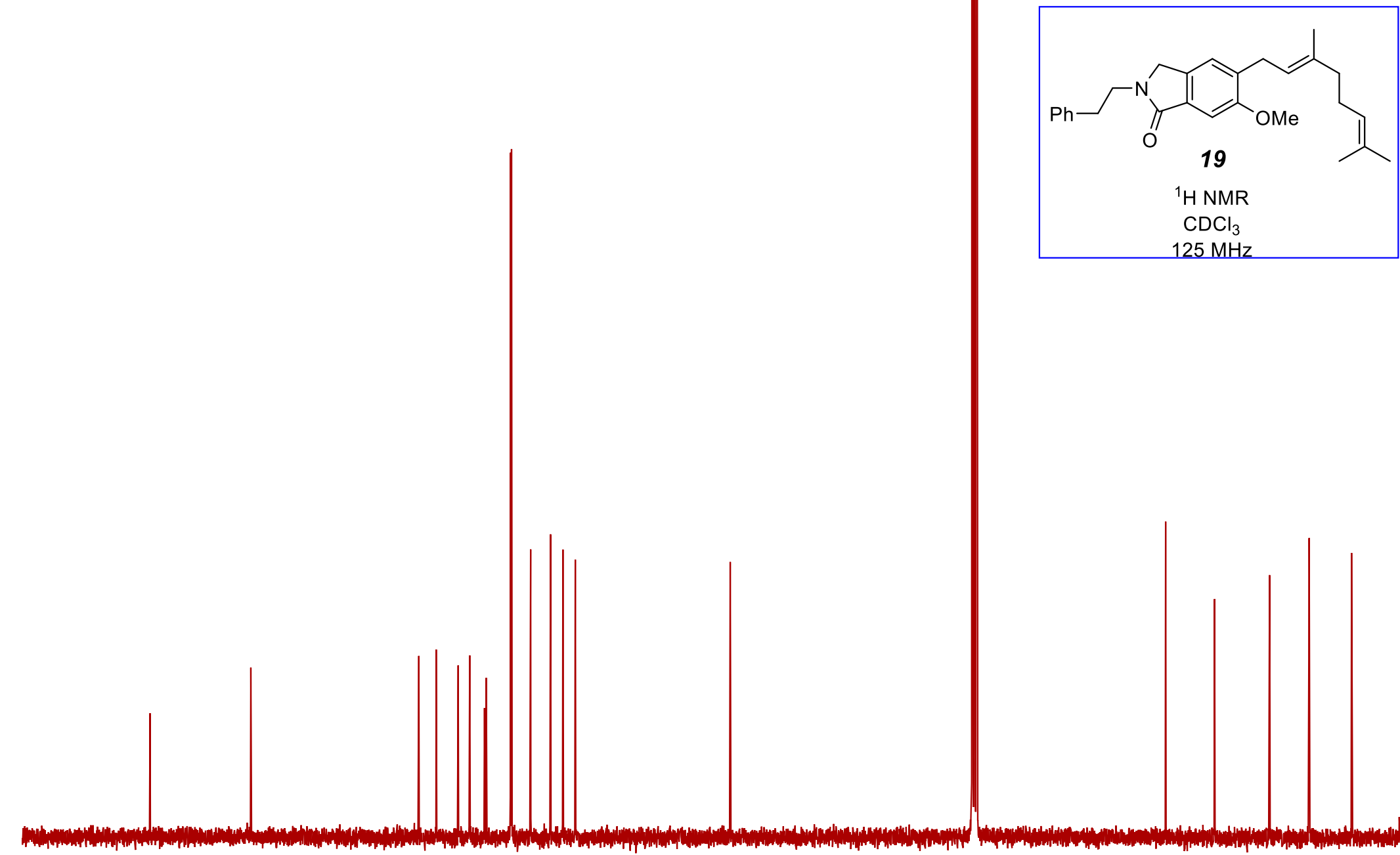


\section{$\mathrm{S} 5 / \mathrm{H} \stackrel{+}{\text { iे }}$}

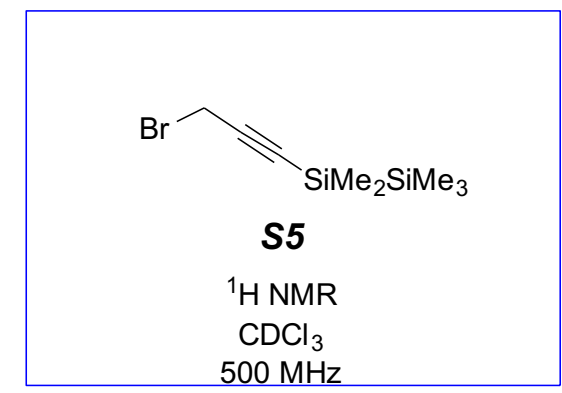

กิ $\frac{m}{0}$

$\begin{array}{ll}0 & 0 \\ 1 & 1\end{array}$

$500 \mathrm{MHz}$

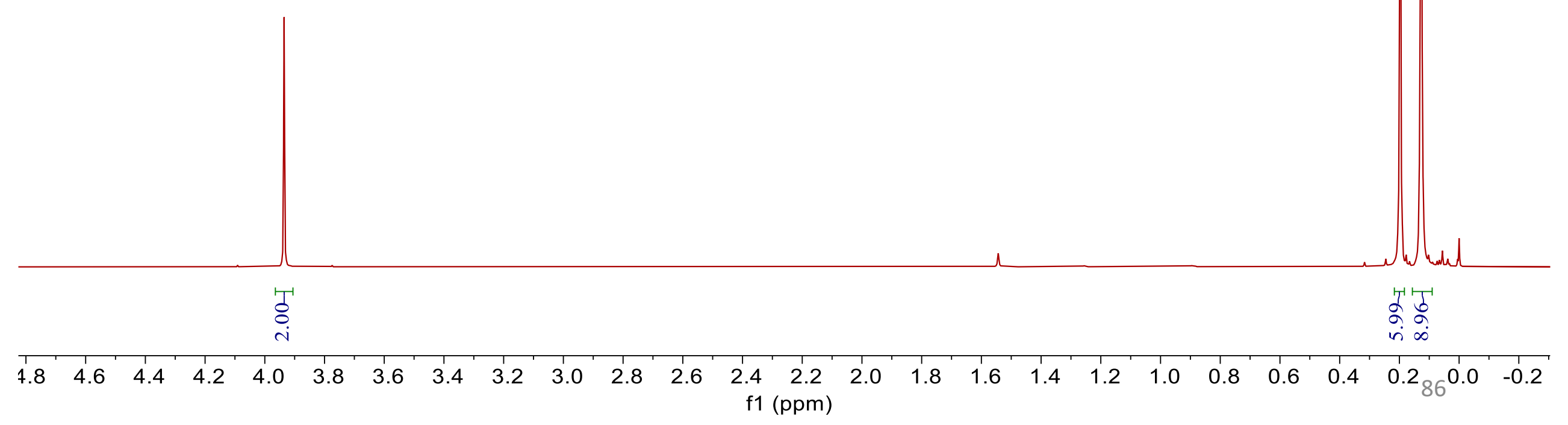




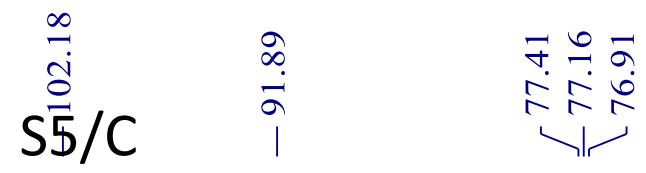
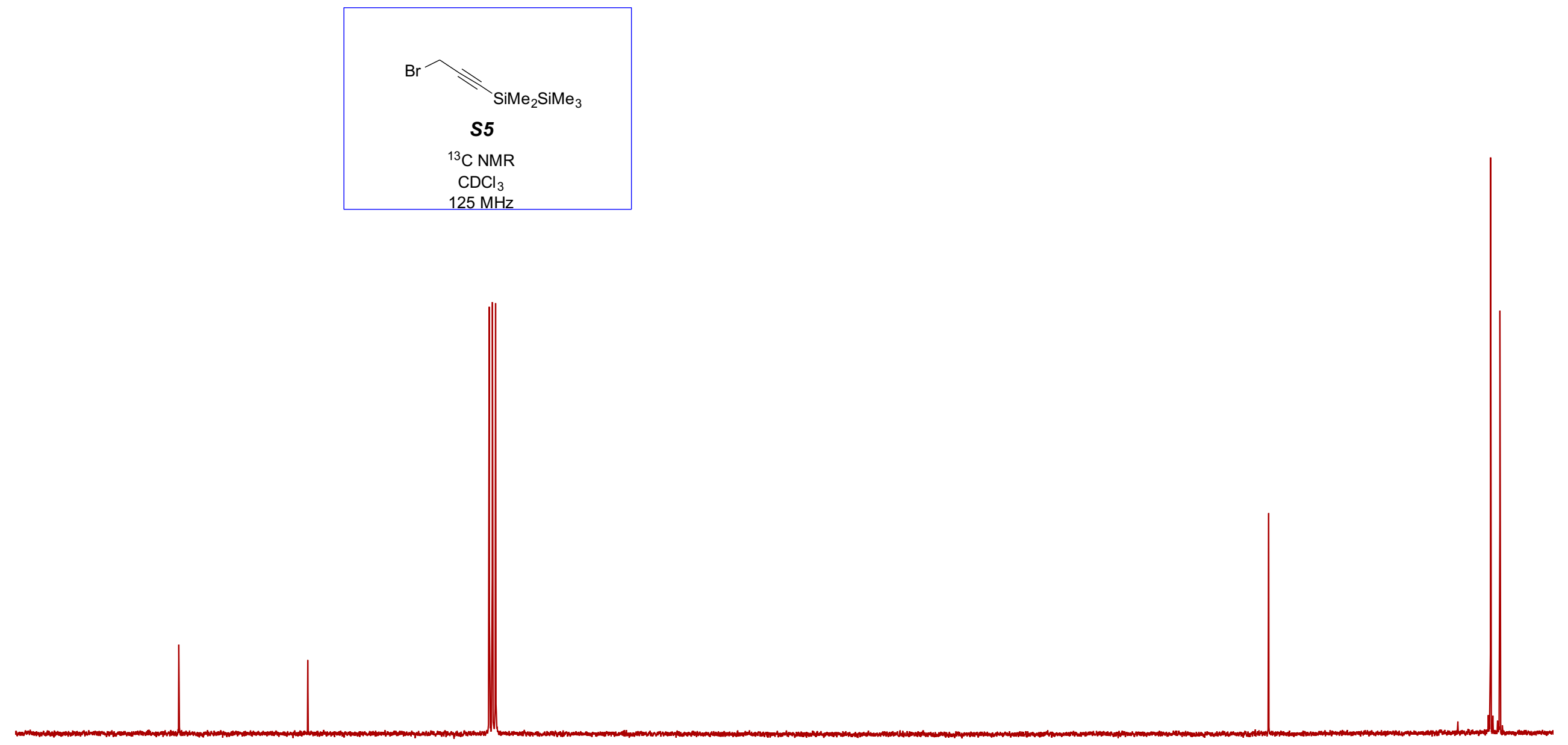

\begin{tabular}{|c|c|c|c|c|c|c|c|c|c|c|c|c|c|c|c|c|c|c|c|c|c|c|c|}
\hline 15 & 110 & 105 & 100 & 95 & 90 & 85 & 80 & 75 & 70 & 65 & 60 & $\begin{array}{r}55 \\
f 1(p p\end{array}$ & $\begin{array}{l}50 \\
\mathrm{n})\end{array}$ & 45 & 40 & 35 & 30 & 25 & 20 & 15 & 10 & ${ }^{5} 87^{0}$ & -5 \\
\hline
\end{tabular}




\section{$\mathrm{S} 6 / \mathrm{H}$}

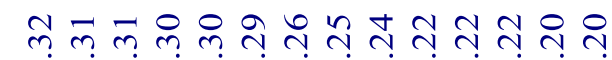

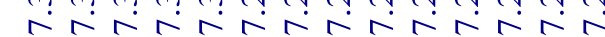
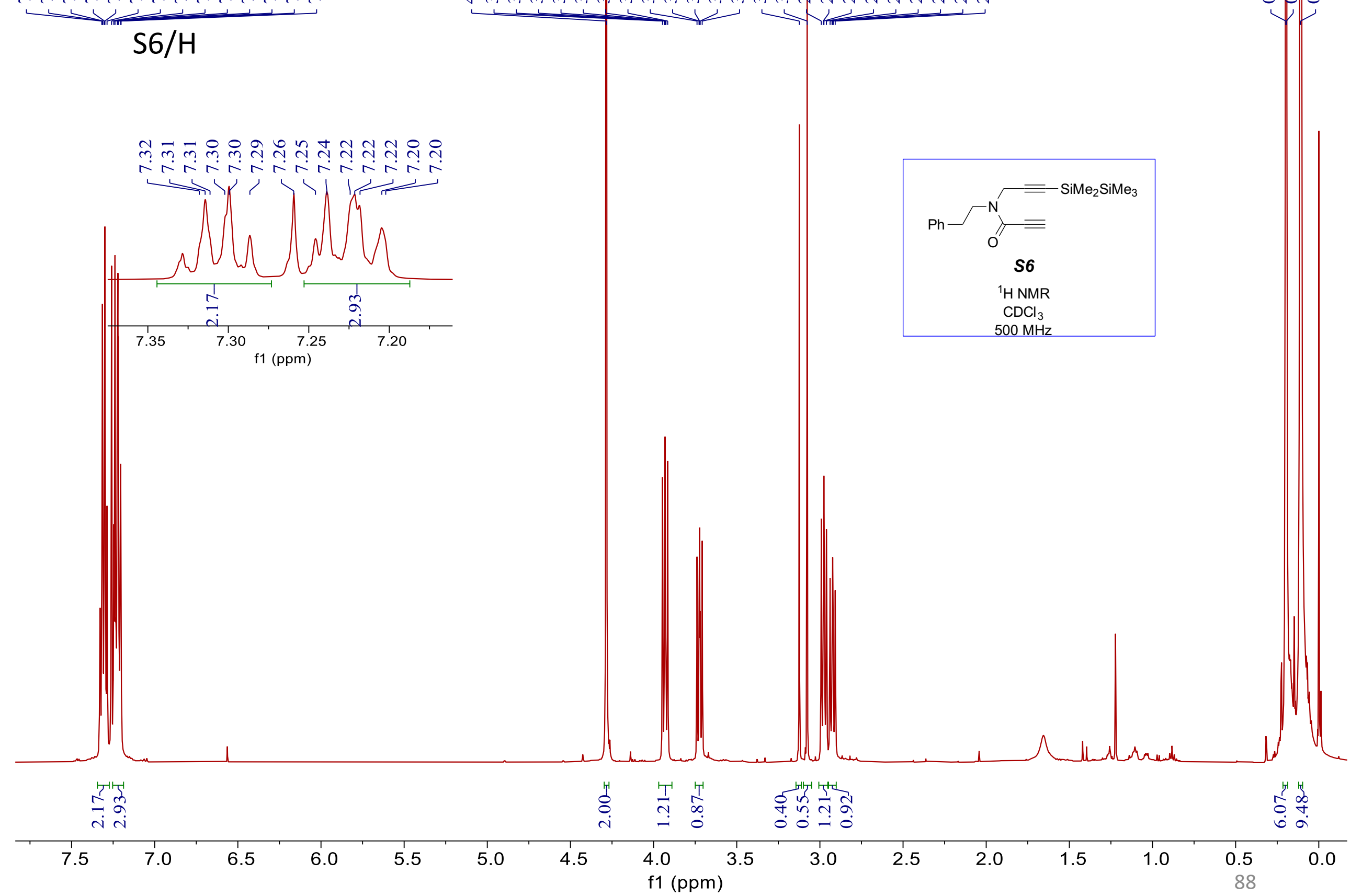

f1 (ppm)

$S 80$ of $S 139$ 


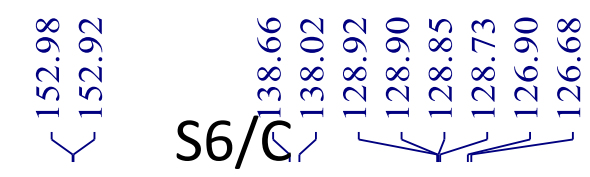

$$
\text { ํㅗำ }
$$

구 ㅇำ

के $\dot{+} \dot{\sim} \dot{m}$

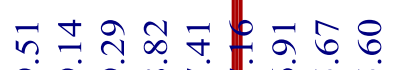

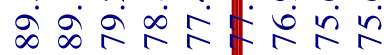

। । लिए।
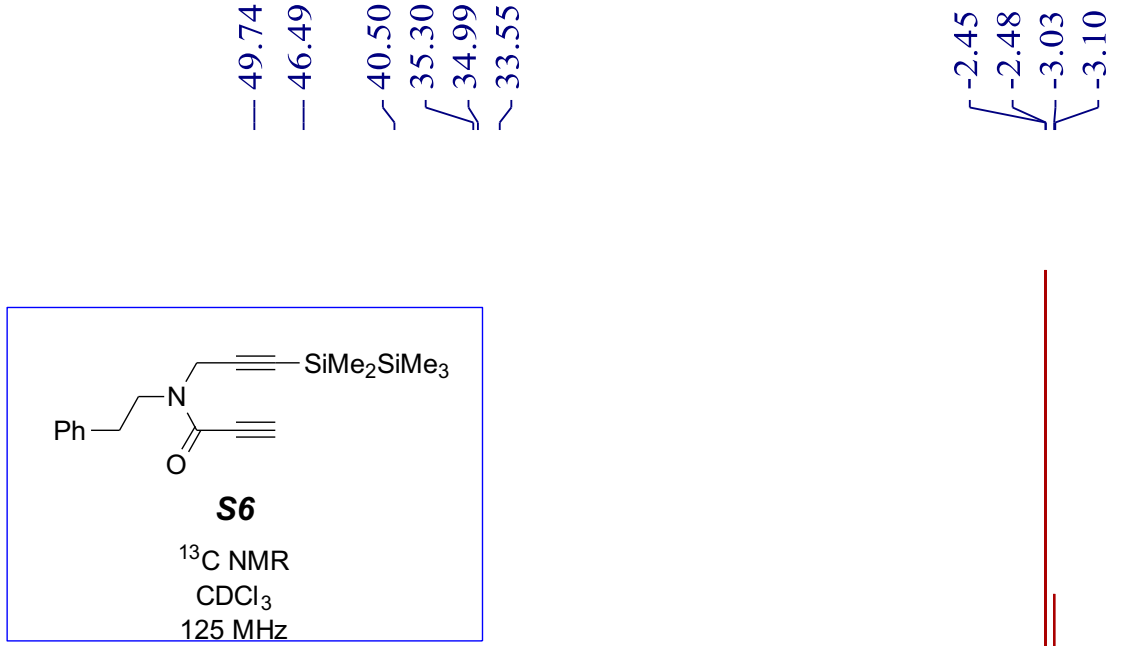

129

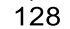

f1 (ppm)

å

它

i $\alpha={ }^{\infty}$

ㄱ. 든
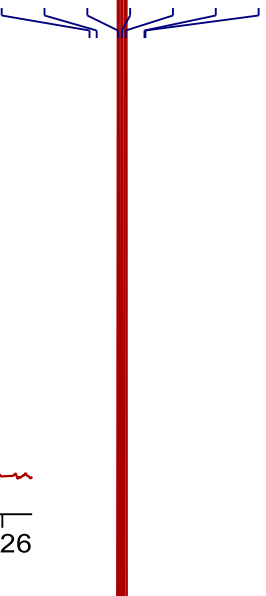

$\longrightarrow$ 


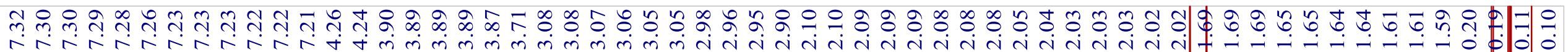
S7/H

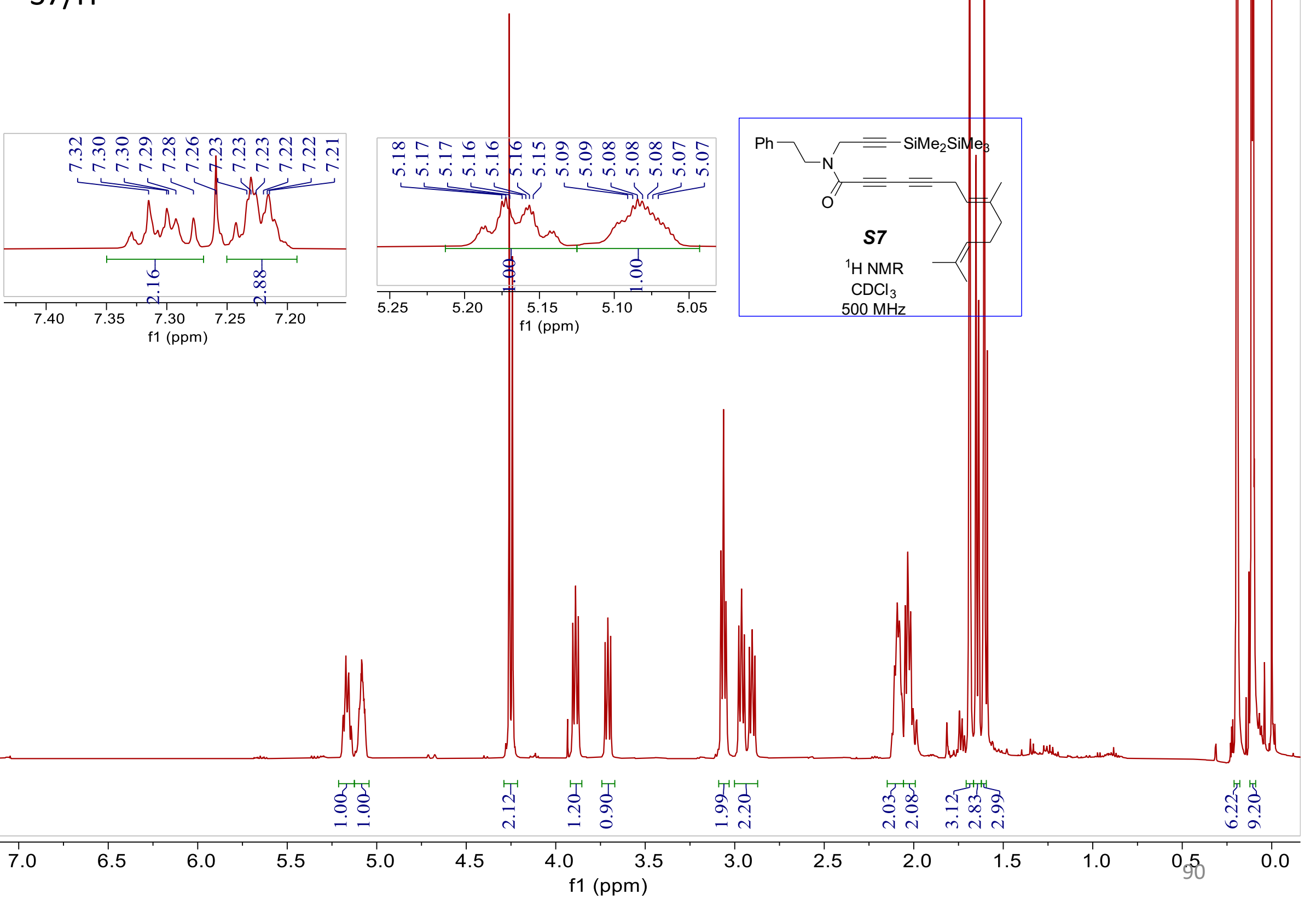

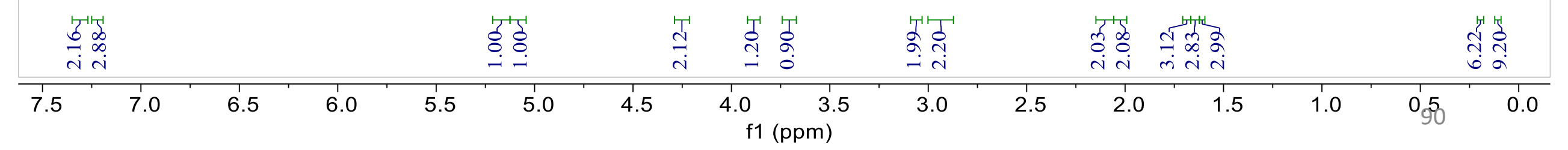




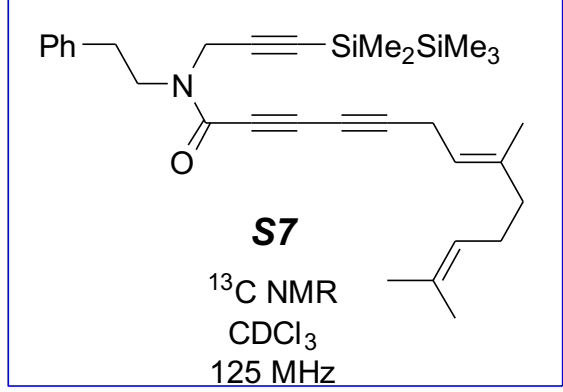




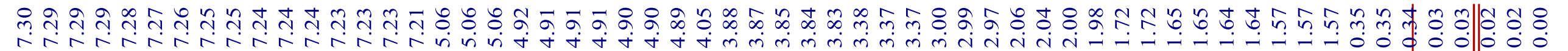

\section{8b/H}

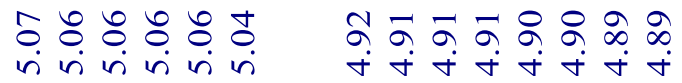

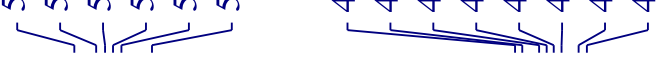
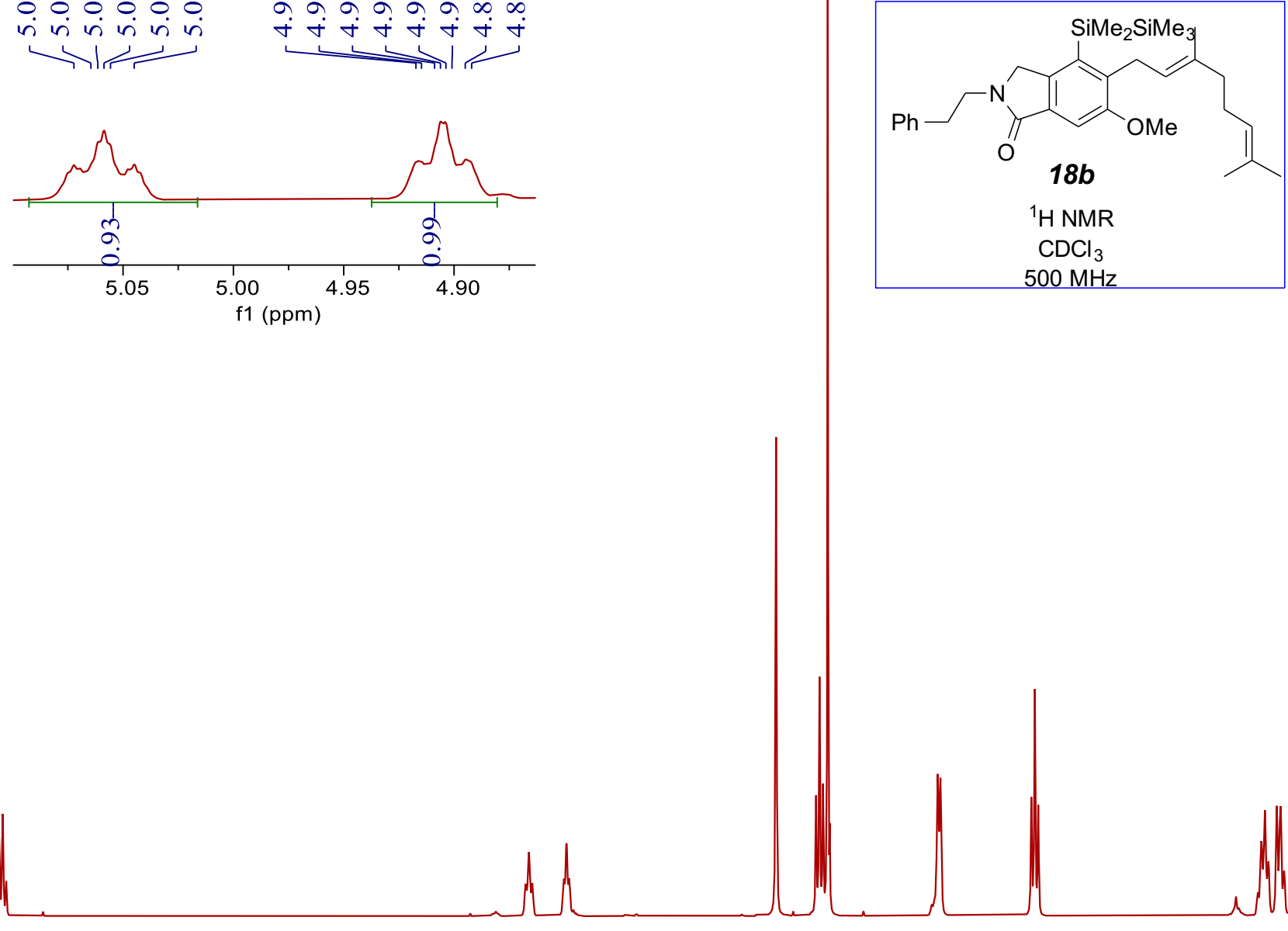

N
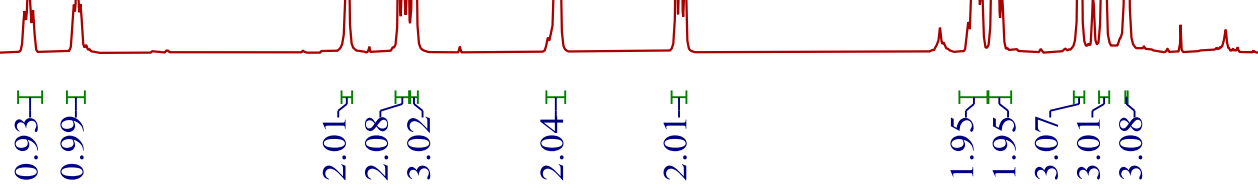

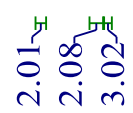

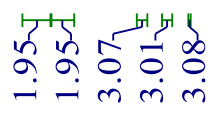

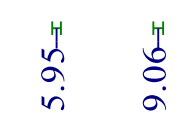

7.5

7.0

6.5

6.0

5.5

$5.0 \quad 4.5$

4.0

f1 (ppm)

$3.0 \quad 2.5$

20

1.5

92 


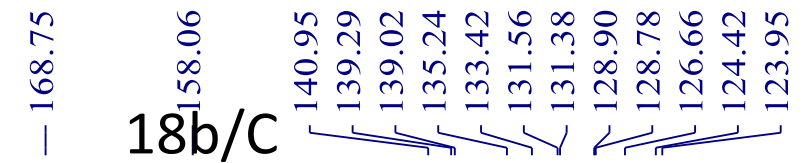

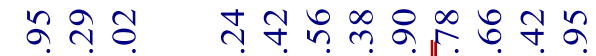

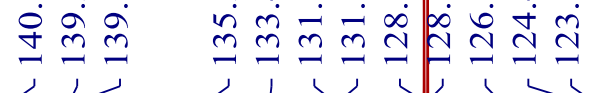

11
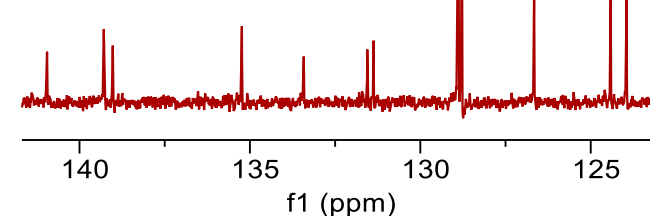

$\stackrel{n}{r}$

in
귕ำ 유요

मेंñ

$\infty 6$

$\stackrel{0}{5}$

11
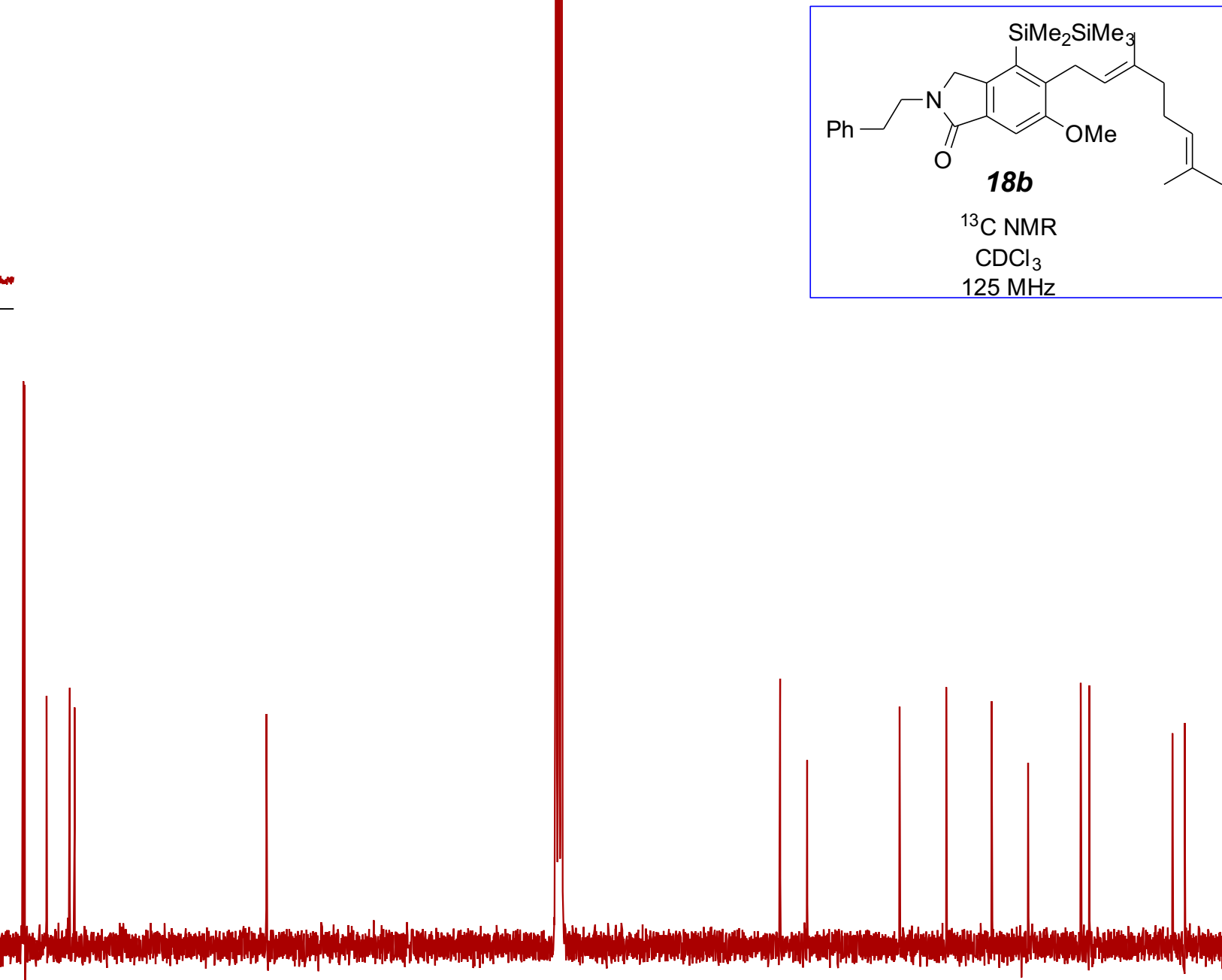


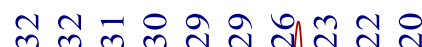

minnsinis
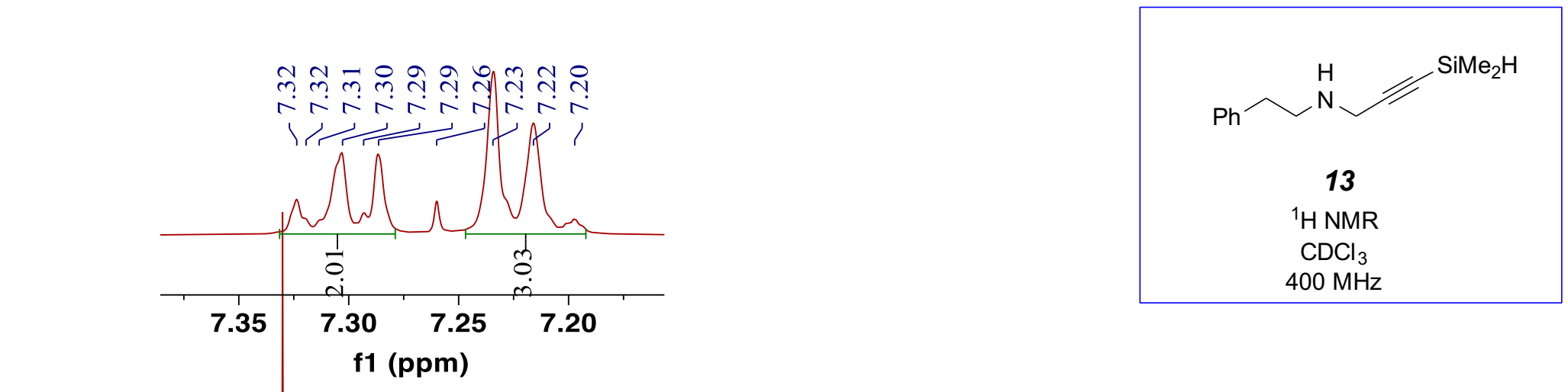

${ }^{1} \mathrm{H} \mathrm{NMR}$
$\mathrm{CDCl}_{3}$
$400 \mathrm{MHz}$

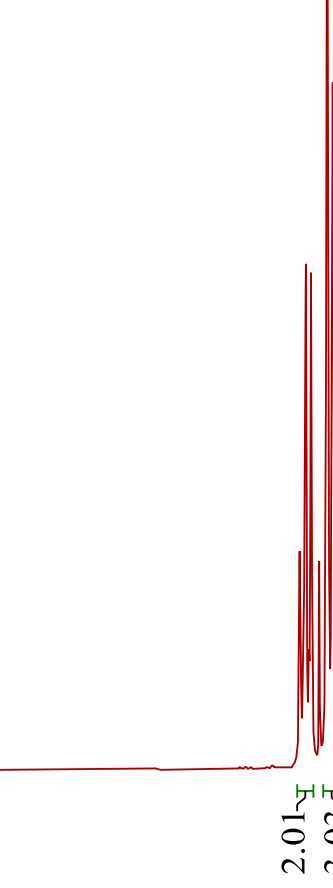

8.0

7.5

7.0

6.5

6.0

f1 (ppm)
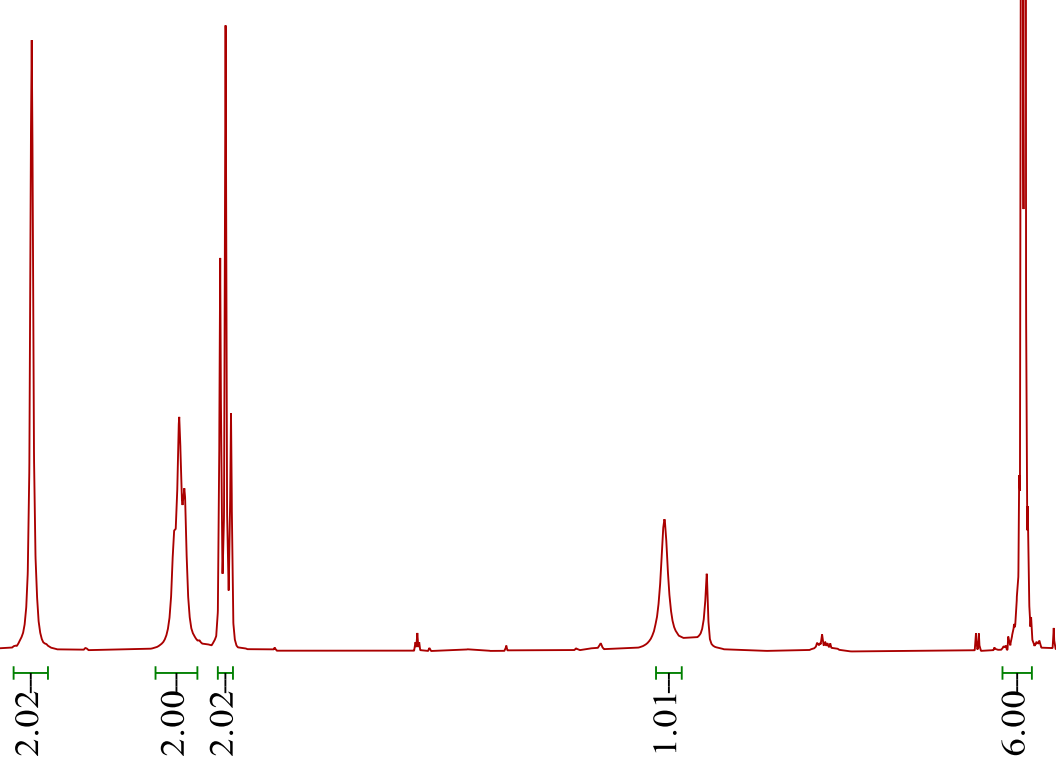

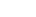

1

2.5

2.0

1.5

$1.0 \quad 940.5$ 


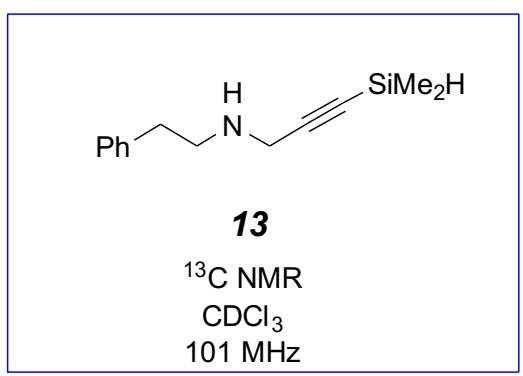

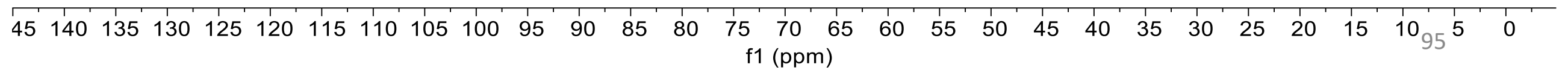




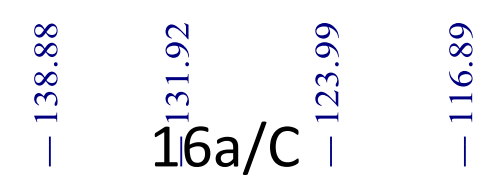
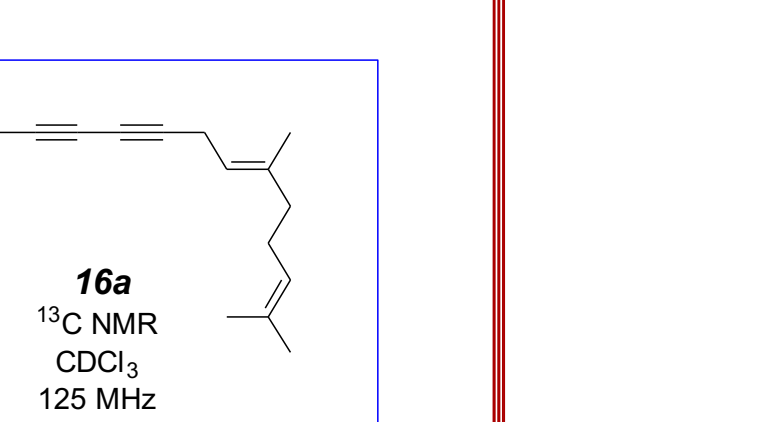


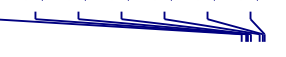

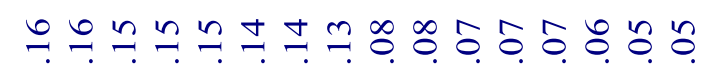
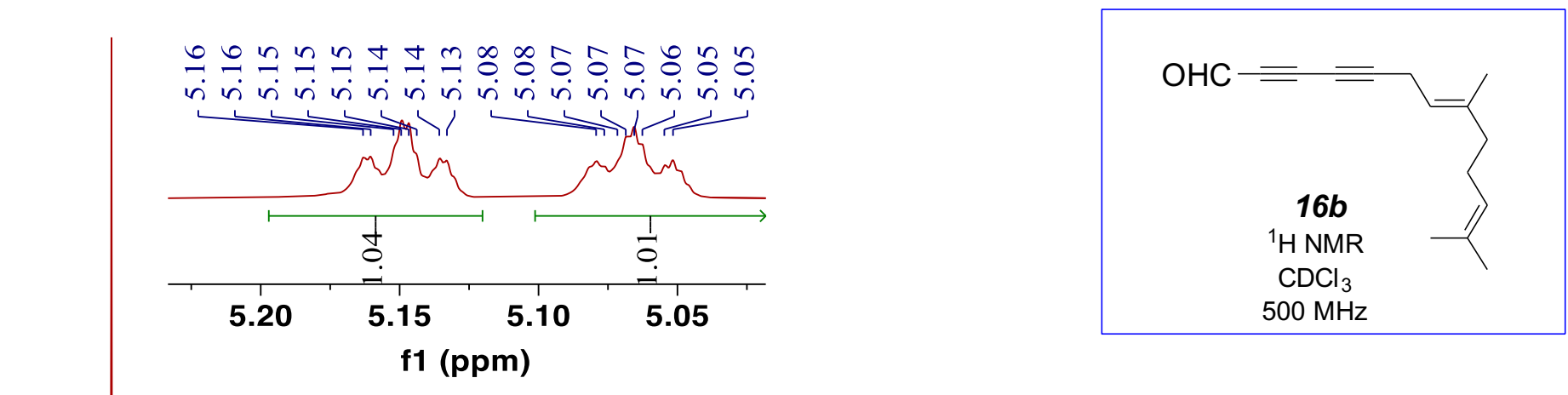

f1 (ppm)
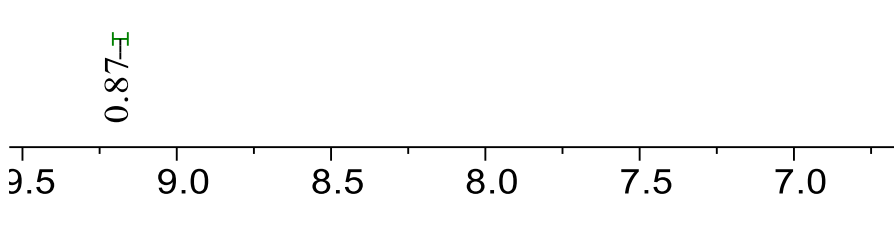

\section{坐产}

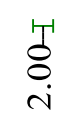

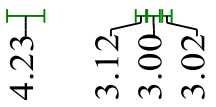
f1 (ppm)

$\begin{array}{lllllll}4.5 & 4.0 & 3.5 & 3.0 & 2.5 & 2.98 & 1.5\end{array}$




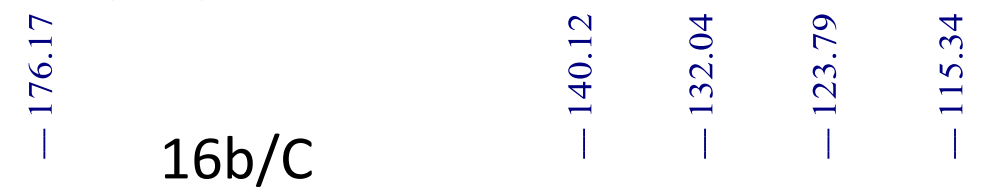

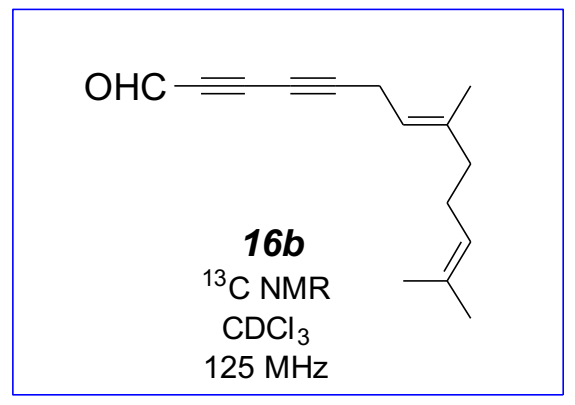

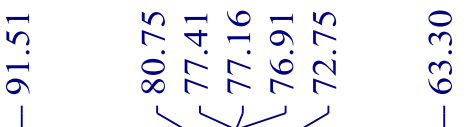


드느는
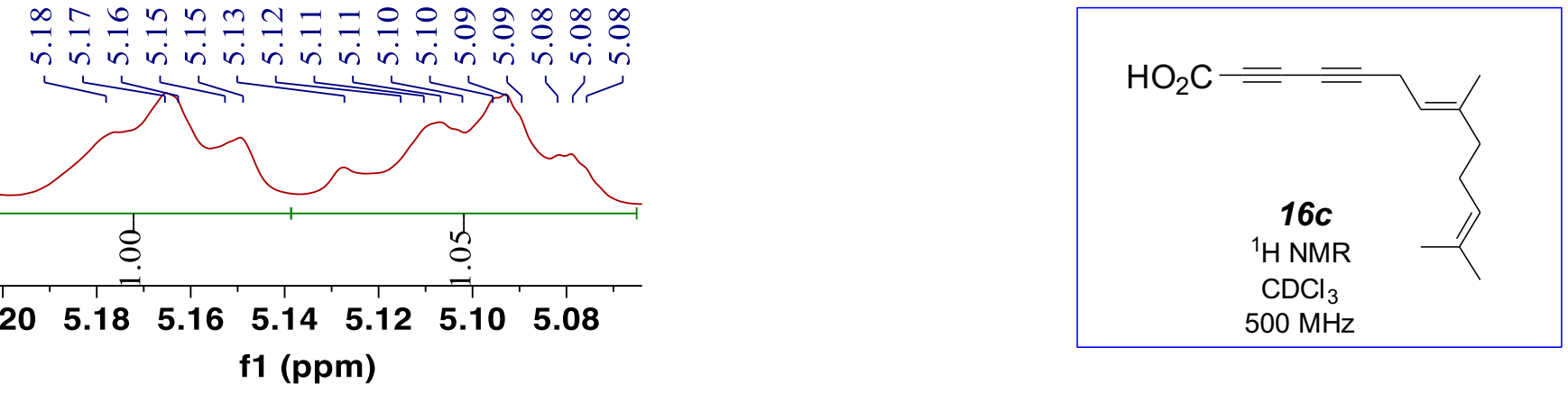

f1 (ppm)

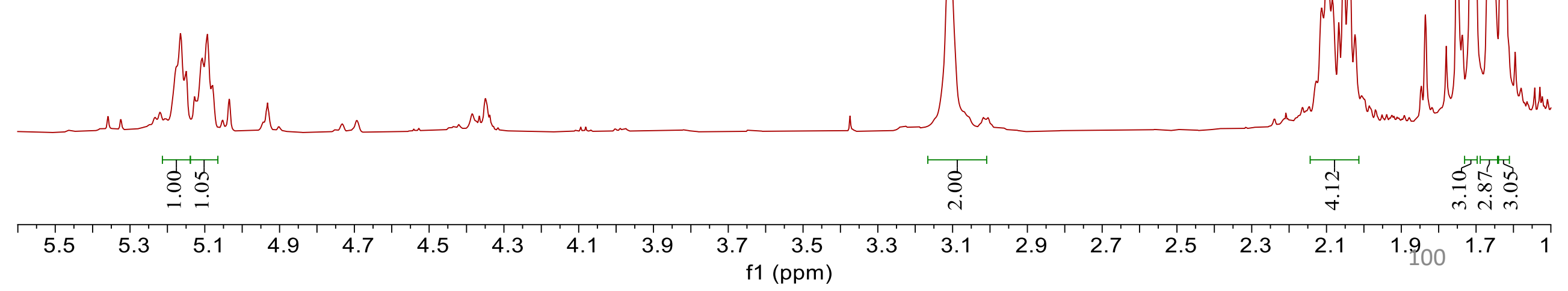




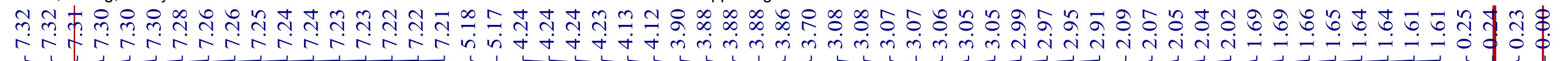

\section{$17 / \mathrm{H}$}

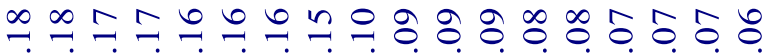

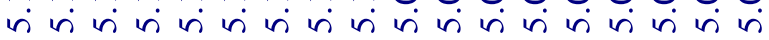
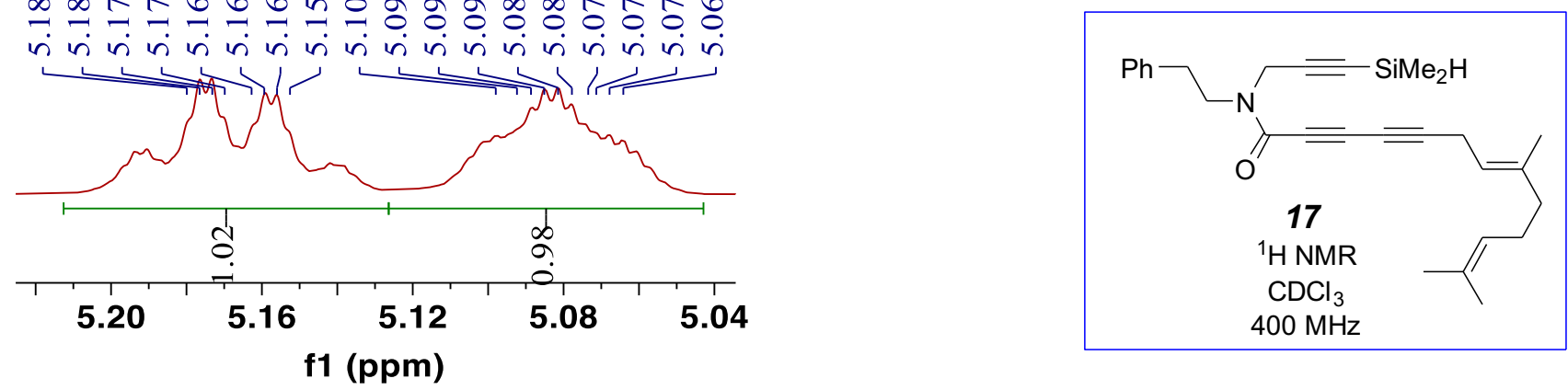

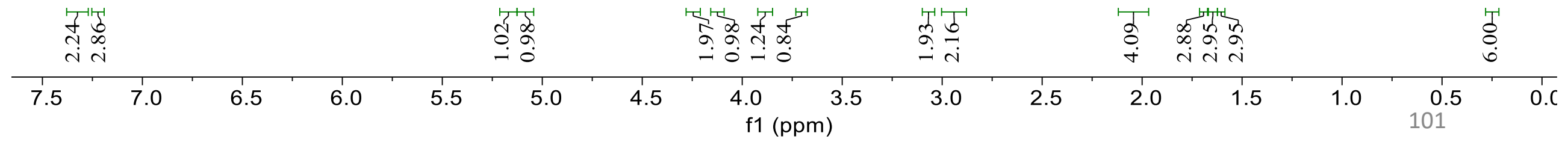




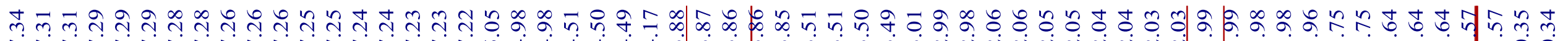
$18 \mathrm{c} / \mathrm{H}$

मे rum
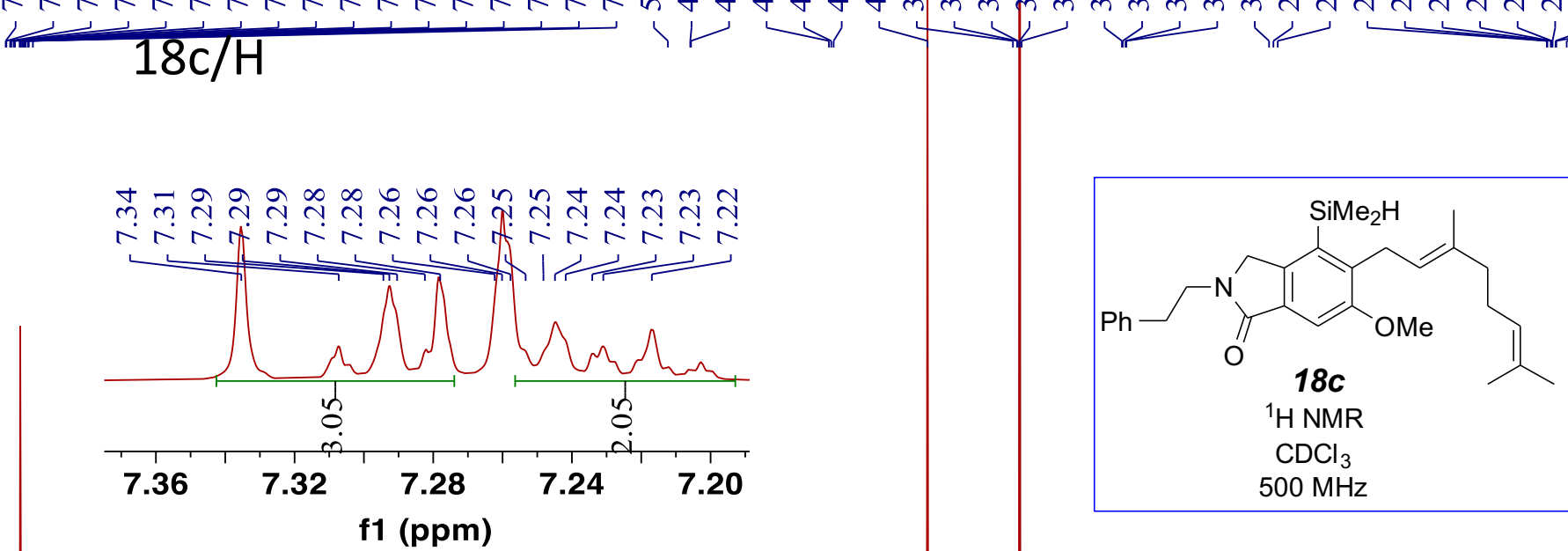
f1 (ppm)
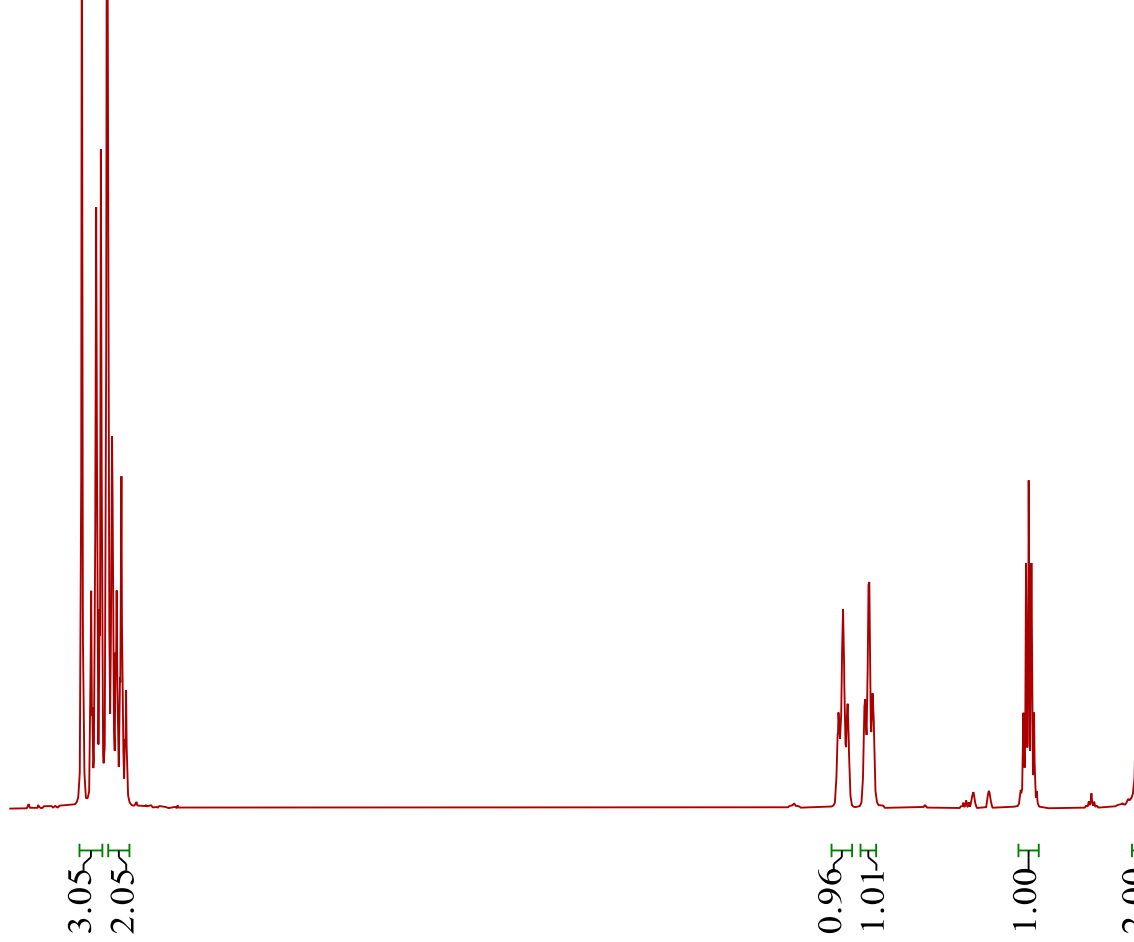

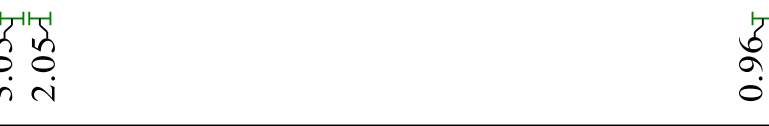

年管

को




\section{$18 c / C$}

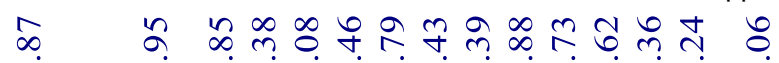

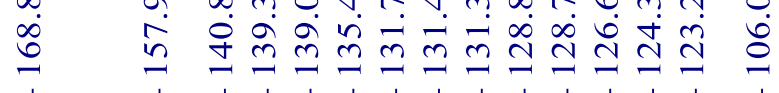

$\infty \infty \quad n \geqslant 0 \infty$

in

| 1

$\infty n$

슝ำ

$\infty$

$\longrightarrow$ ।

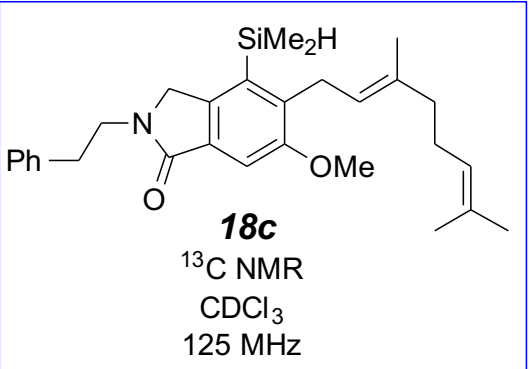

$125 \mathrm{MHz}$

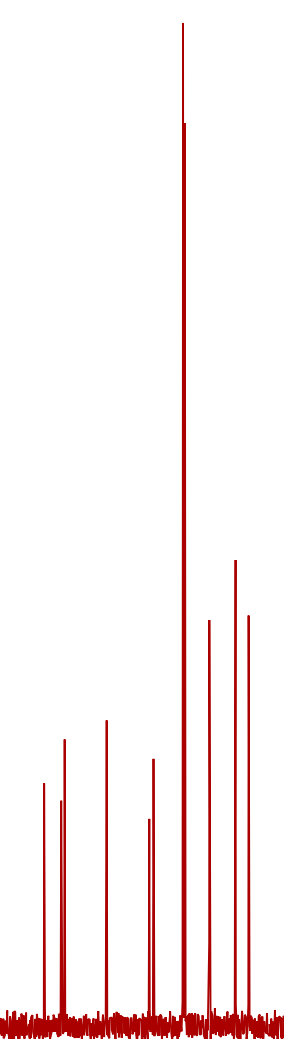

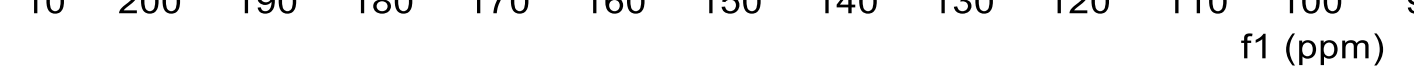

80

70

60

50

40

30

20

10




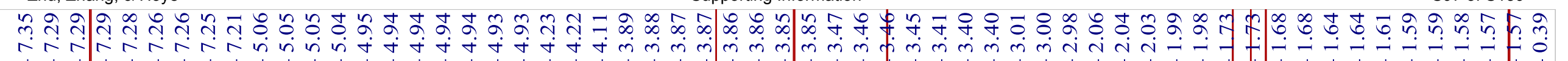

\section{$18 \mathrm{~d} / \mathrm{H}$}
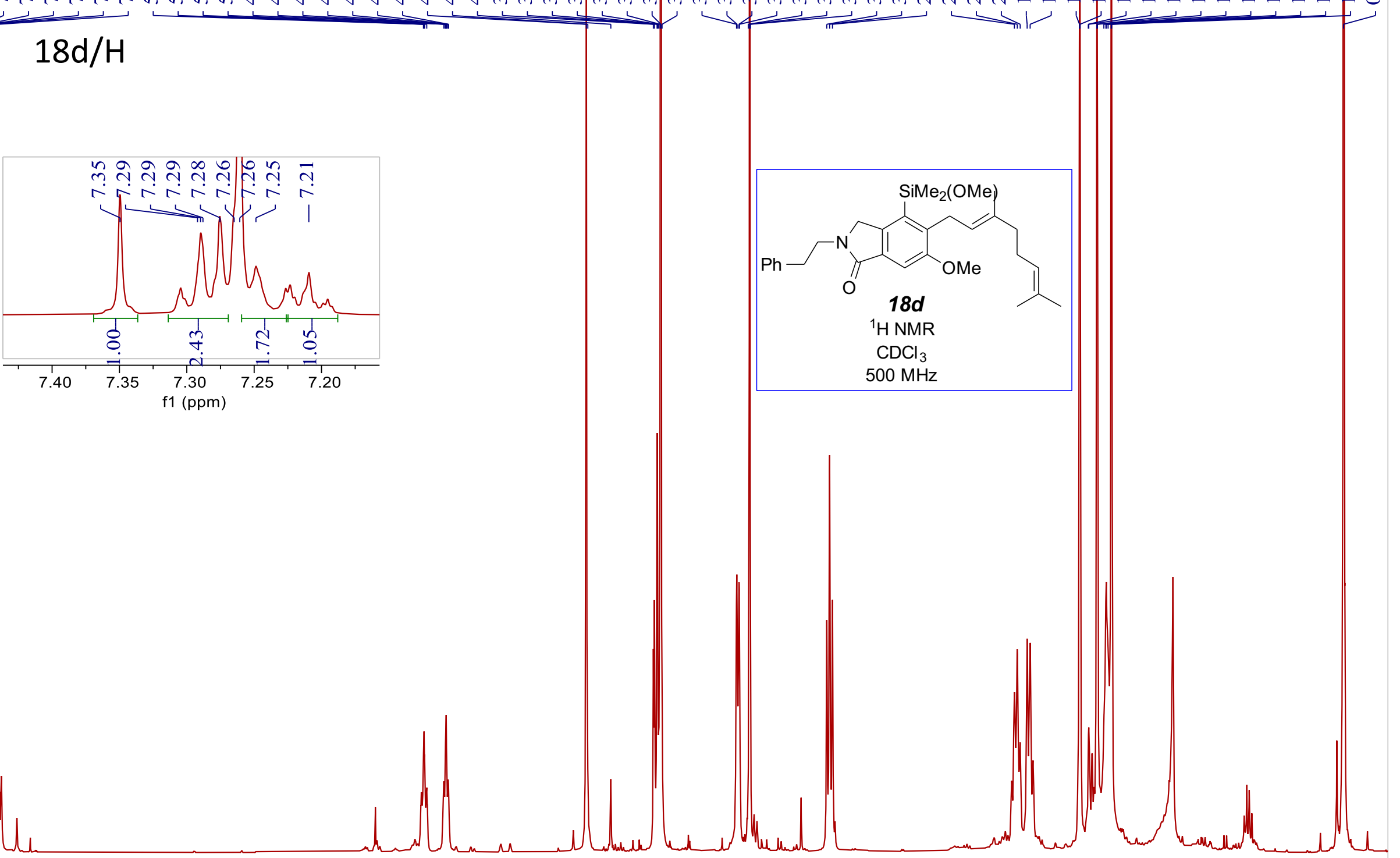

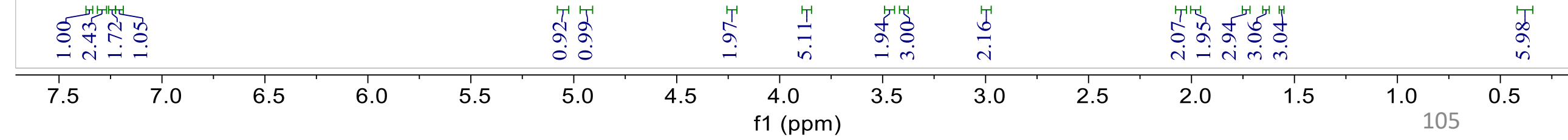


\begin{tabular}{l}
\multirow{2}{*}{$\infty$} \\
$\cdots$
\end{tabular}

in

l1 1
ำกั่ํํ में $<1<1$

$\vec{\infty}+$

$\stackrel{1}{ }$
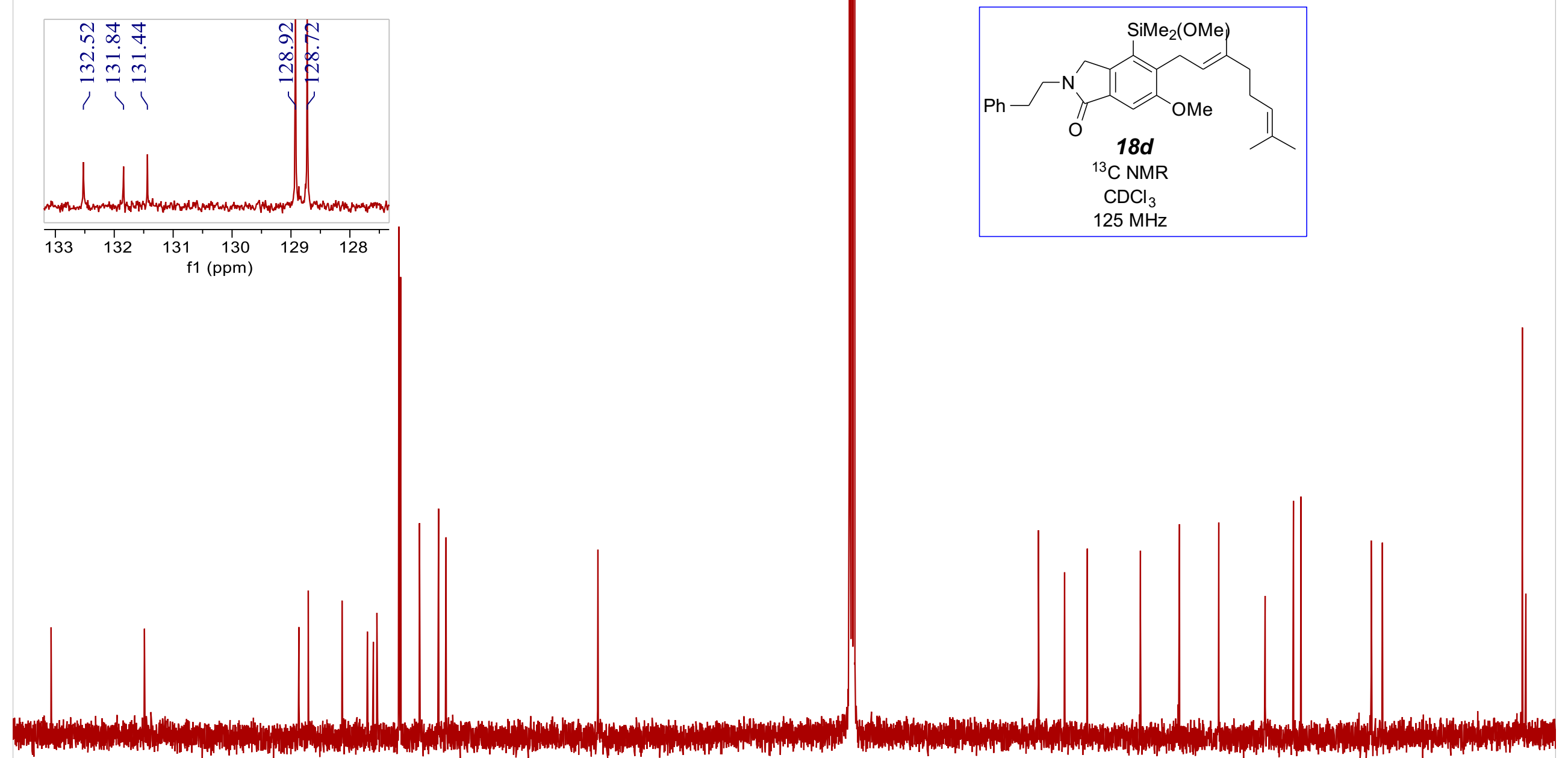

\begin{tabular}{|c|c|c|c|c|c|c|c|c|c|c|c|c|c|c|c|c|c|}
\hline 170 & 160 & 150 & 140 & 130 & 120 & 110 & 100 & $\begin{array}{l}90 \\
\mathrm{f} 1\end{array}$ & $\begin{array}{l}80 \\
80\end{array}$ & 70 & 60 & 50 & 40 & 30 & 20 & 106 & 0 \\
\hline
\end{tabular}




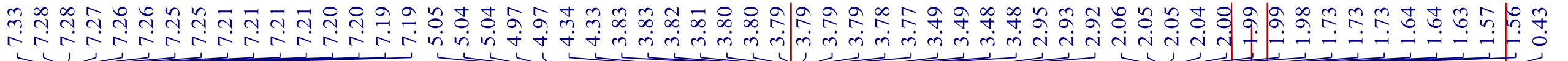

\section{$18 \mathrm{e} / \mathrm{H}$}

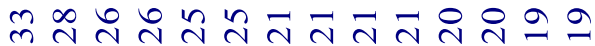

ํำ
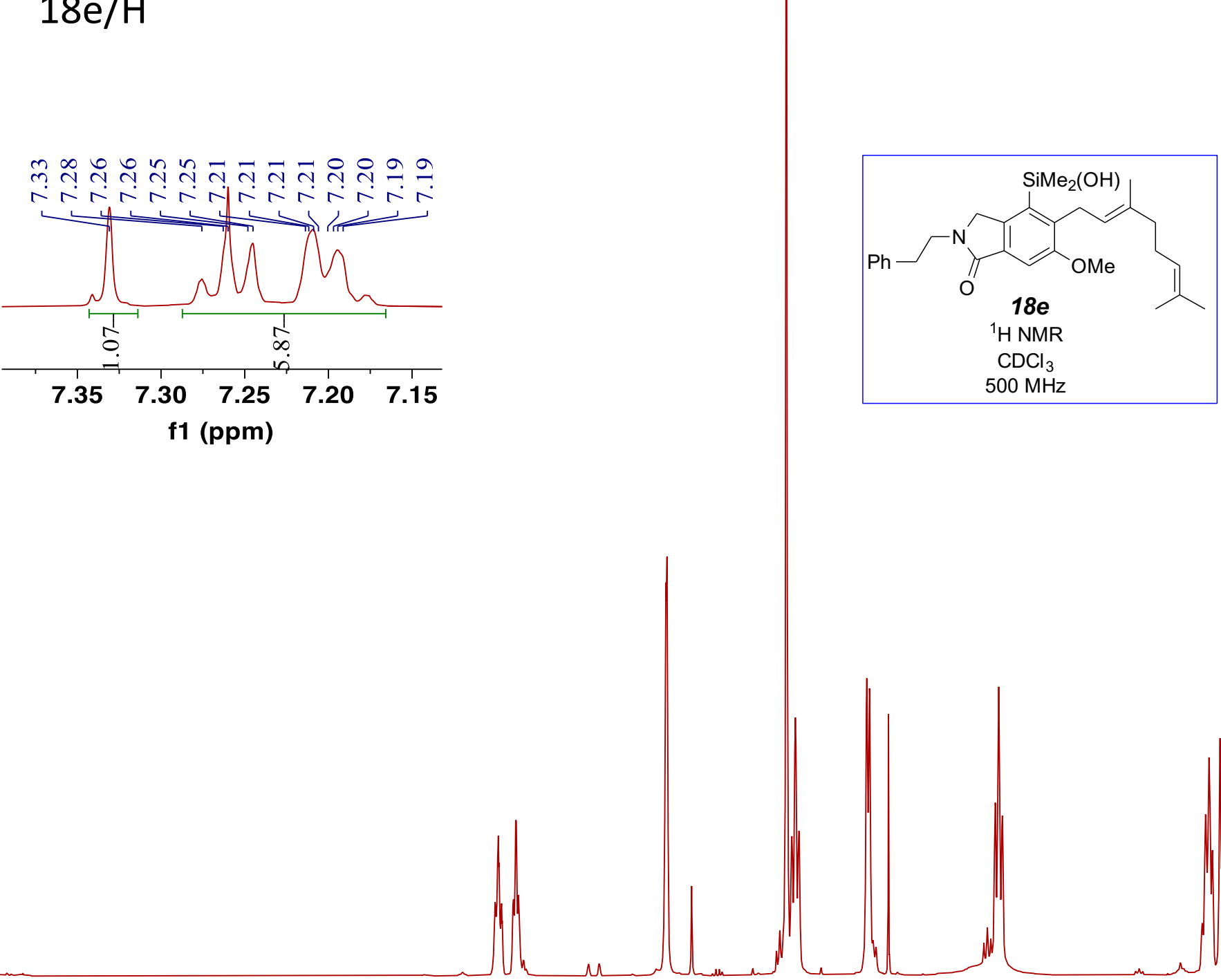

1

\section{坐售}
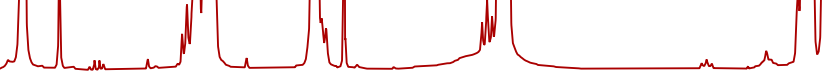

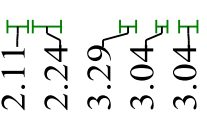

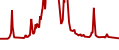

\begin{tabular}{|c|c|c|c|c|c|c|c|}
\hline & & & & & 年管 & $\begin{array}{l}T \\
\stackrel{T}{0} \\
\text { i }\end{array}$ & 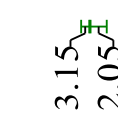 \\
\hline 7.5 & 7.0 & 6.5 & 6.0 & 5.5 & 5.0 & 4.5 & $\begin{array}{l}4.0 \\
1(p p\end{array}$ \\
\hline
\end{tabular}

Th
o
i
3.5

$\stackrel{\substack{4 \\ \text { त }}}{3}$

$3.0 \quad 2.5$

2.0

1.5

$1.0_{107}$

0.5 

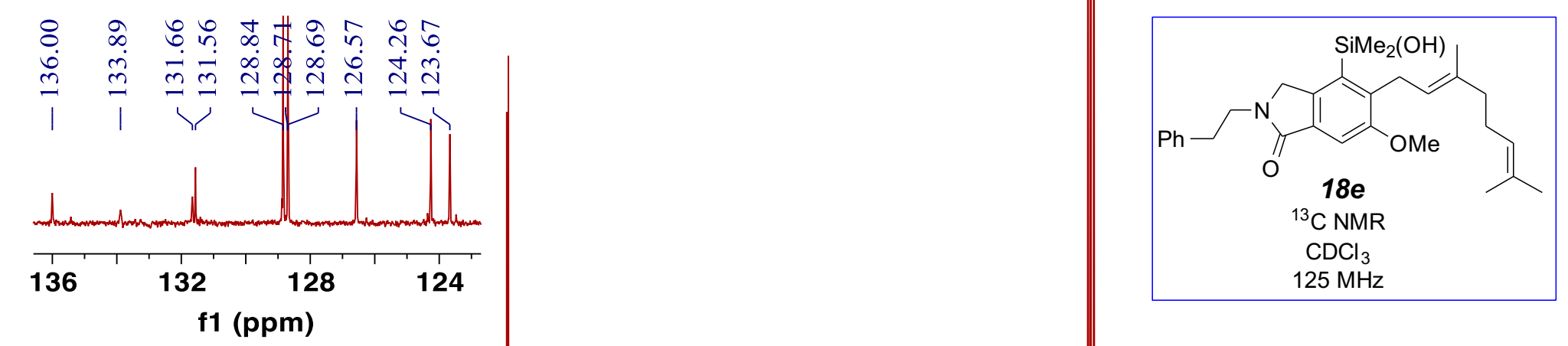

f1 (ppm)

T-




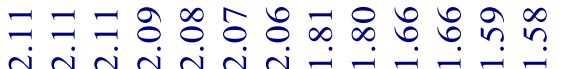
Isohericerin (1)/H

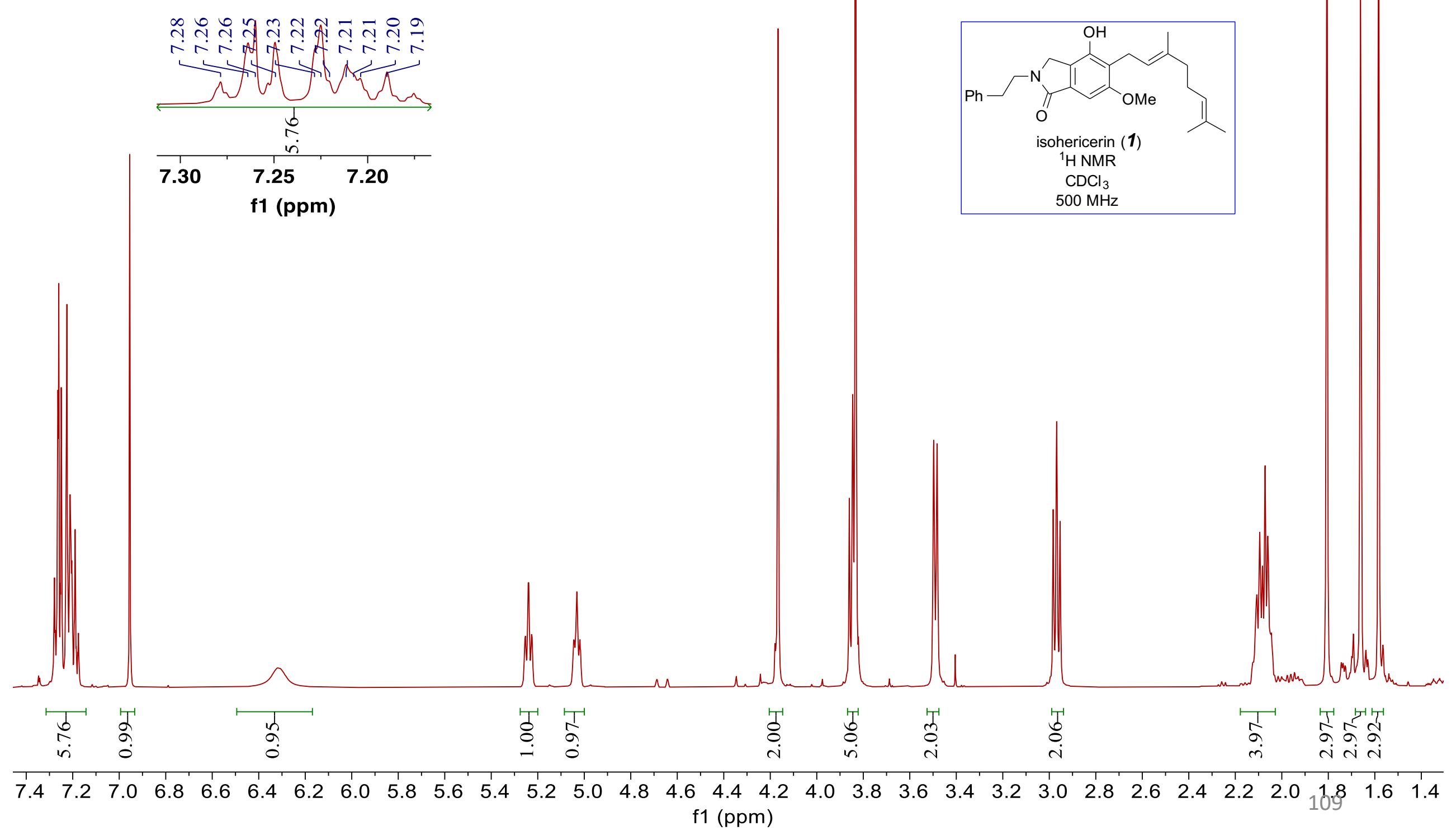




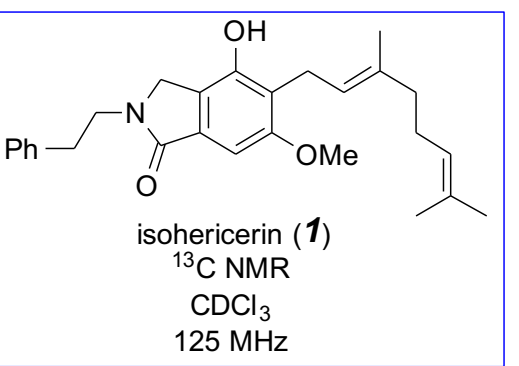

구유 요

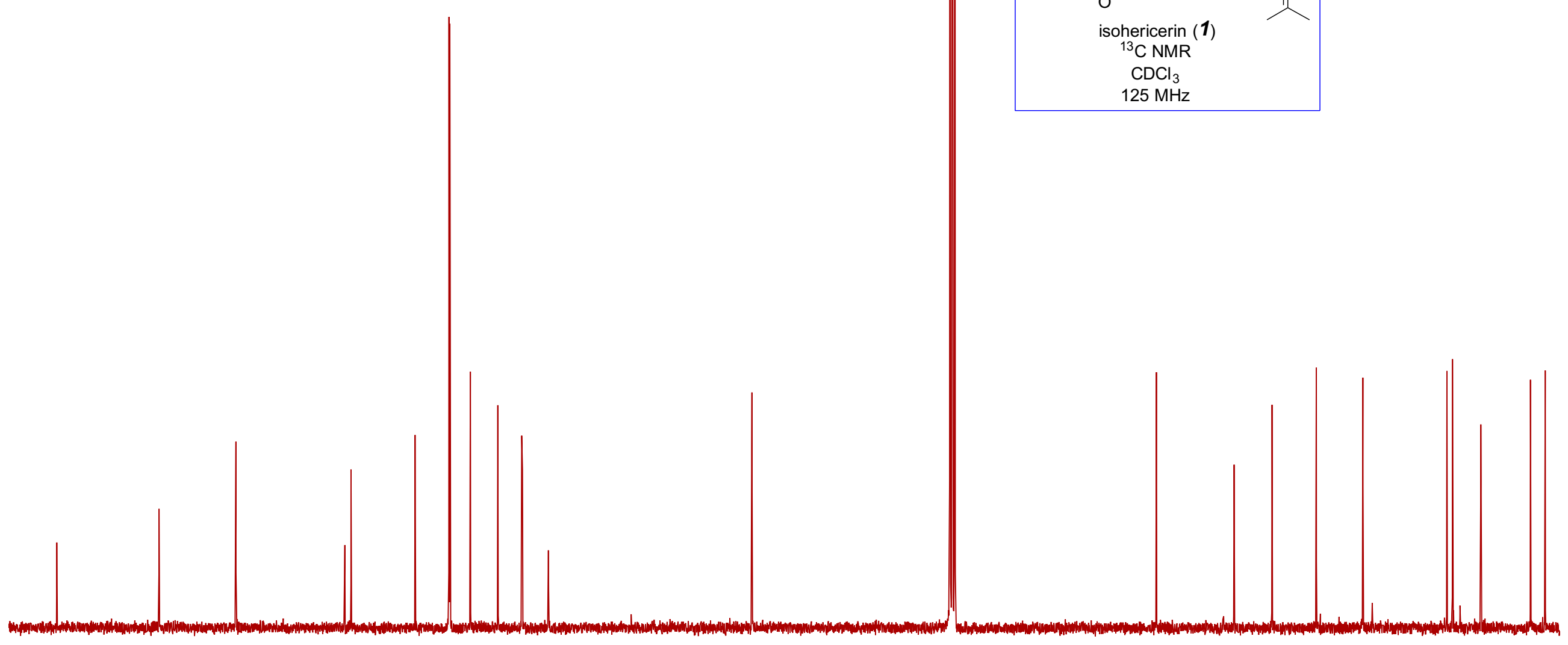

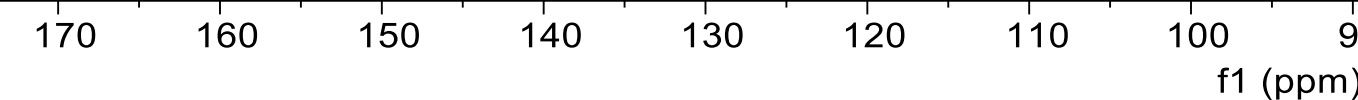

90

80

70

60

50

40

390

20 


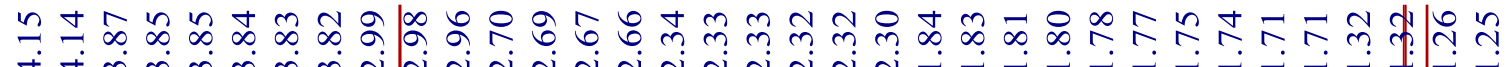

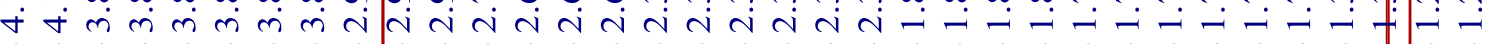

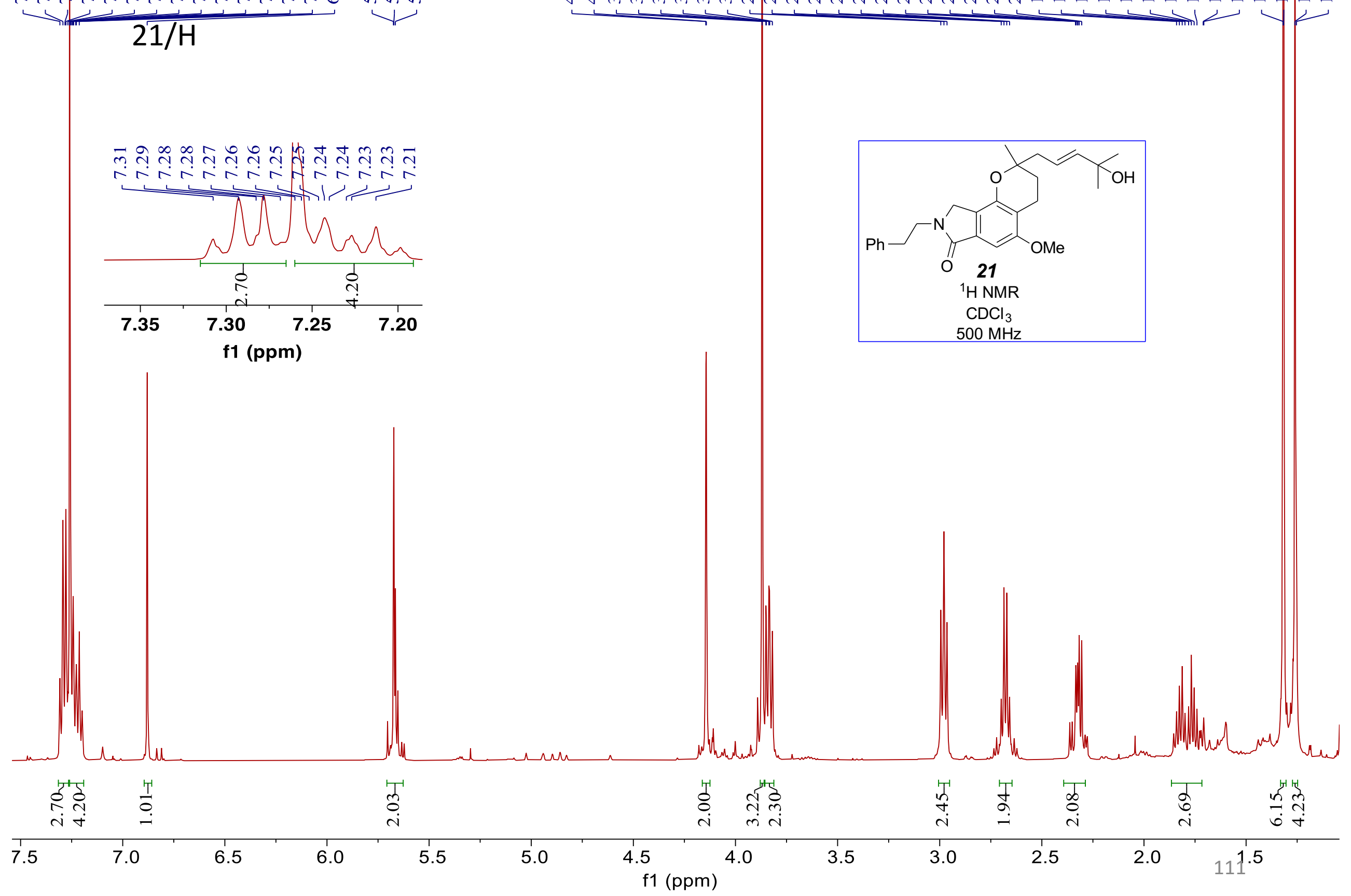



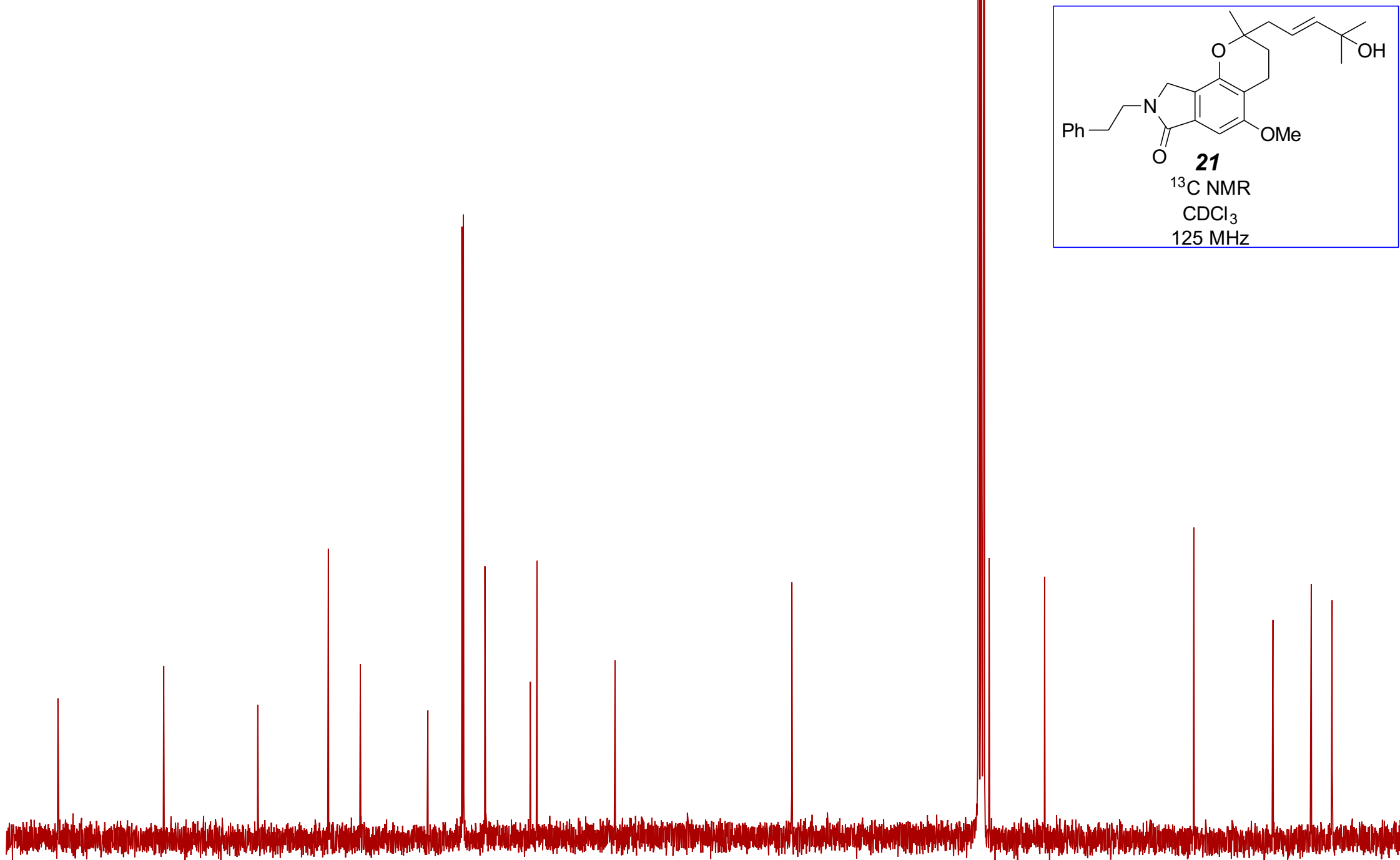
$125 \mathrm{MHz}$

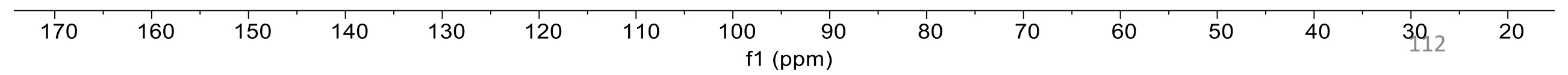




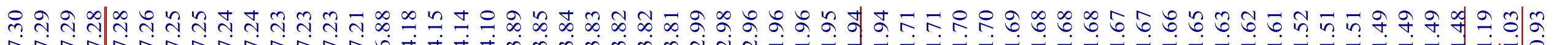
r r r

\section{trans-S8/H}

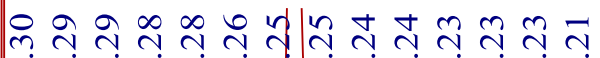

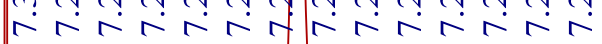

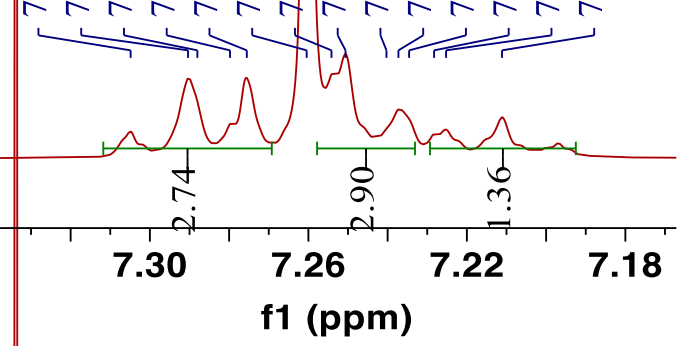

Ma
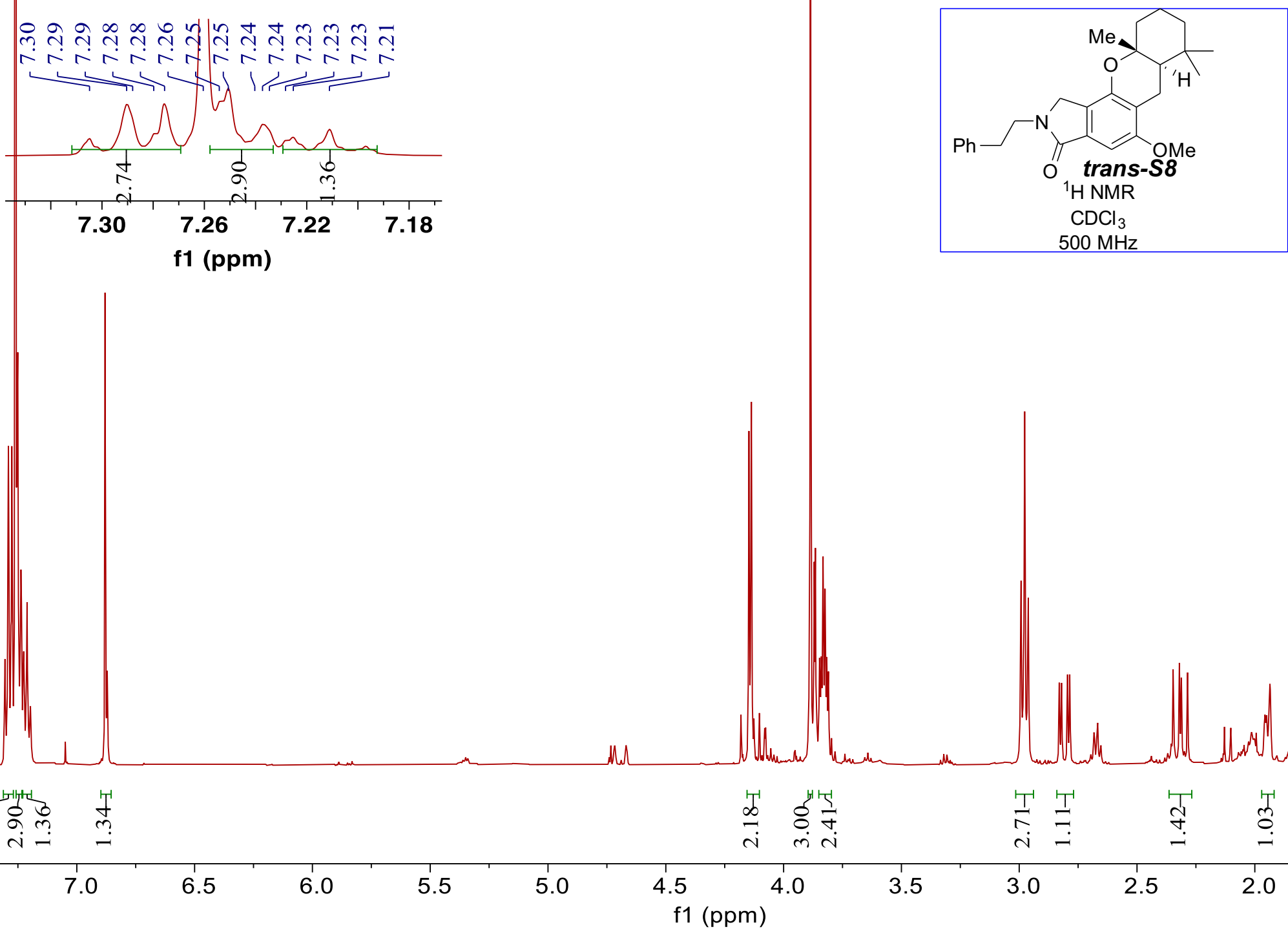

$3.5 \quad 3.0$

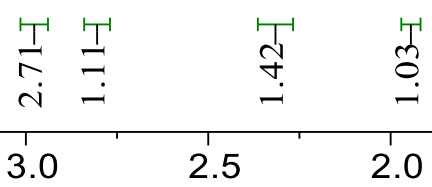

क्षे

$\stackrel{n}{2} \stackrel{n}{2}$

के

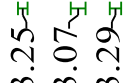

7.5

6.5

6.0

5.5

5.0 


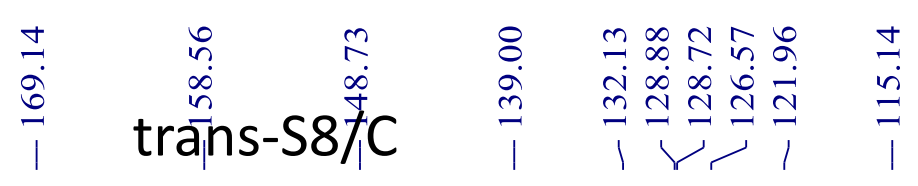

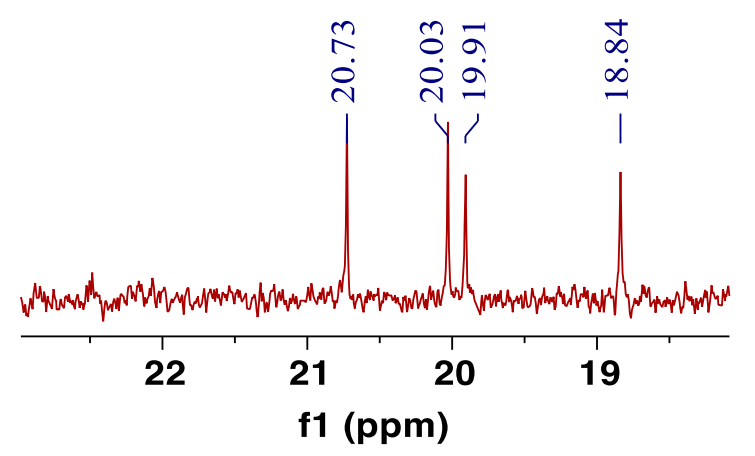

の

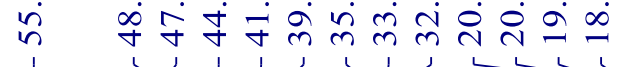

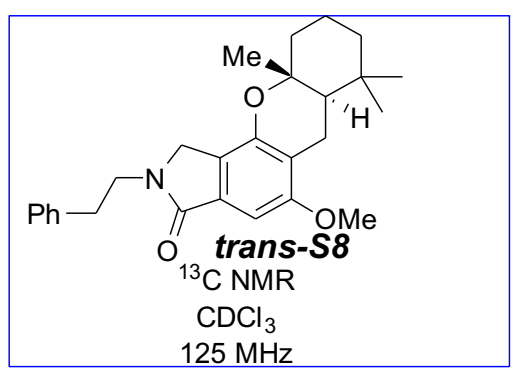

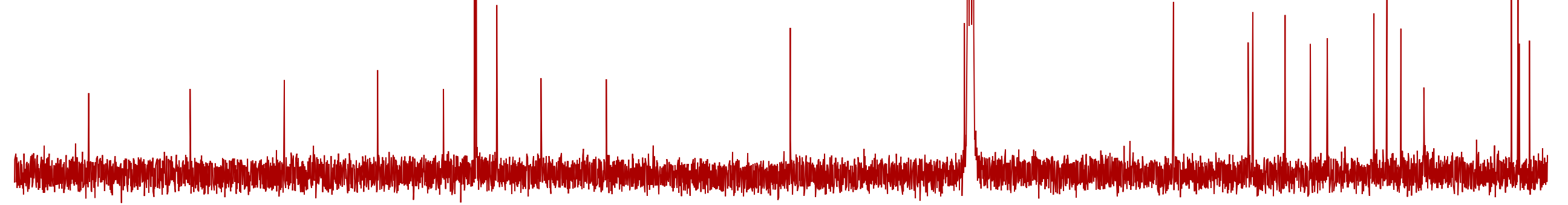




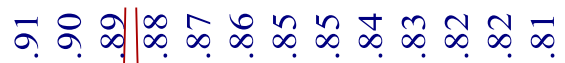

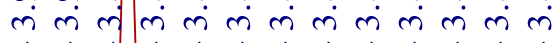

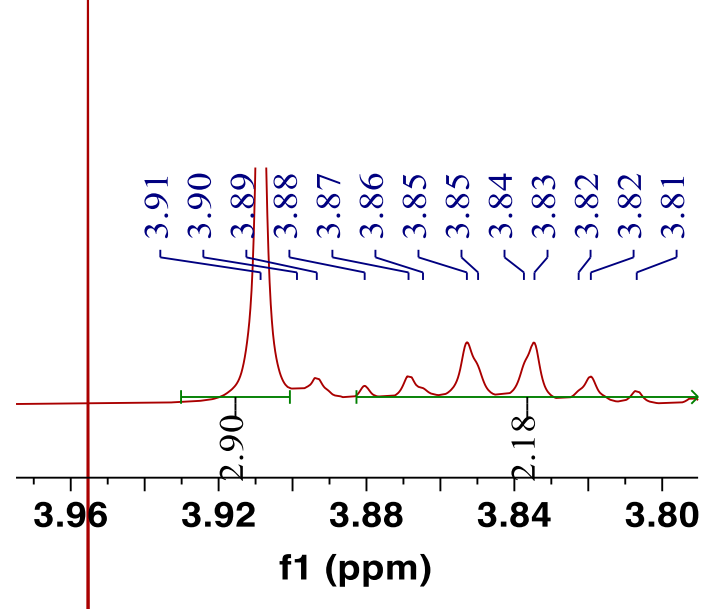

辛究

7.0

6.5

6.0

5.5

5.0

4.5

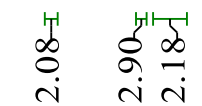

4.0

f1 (ppm)

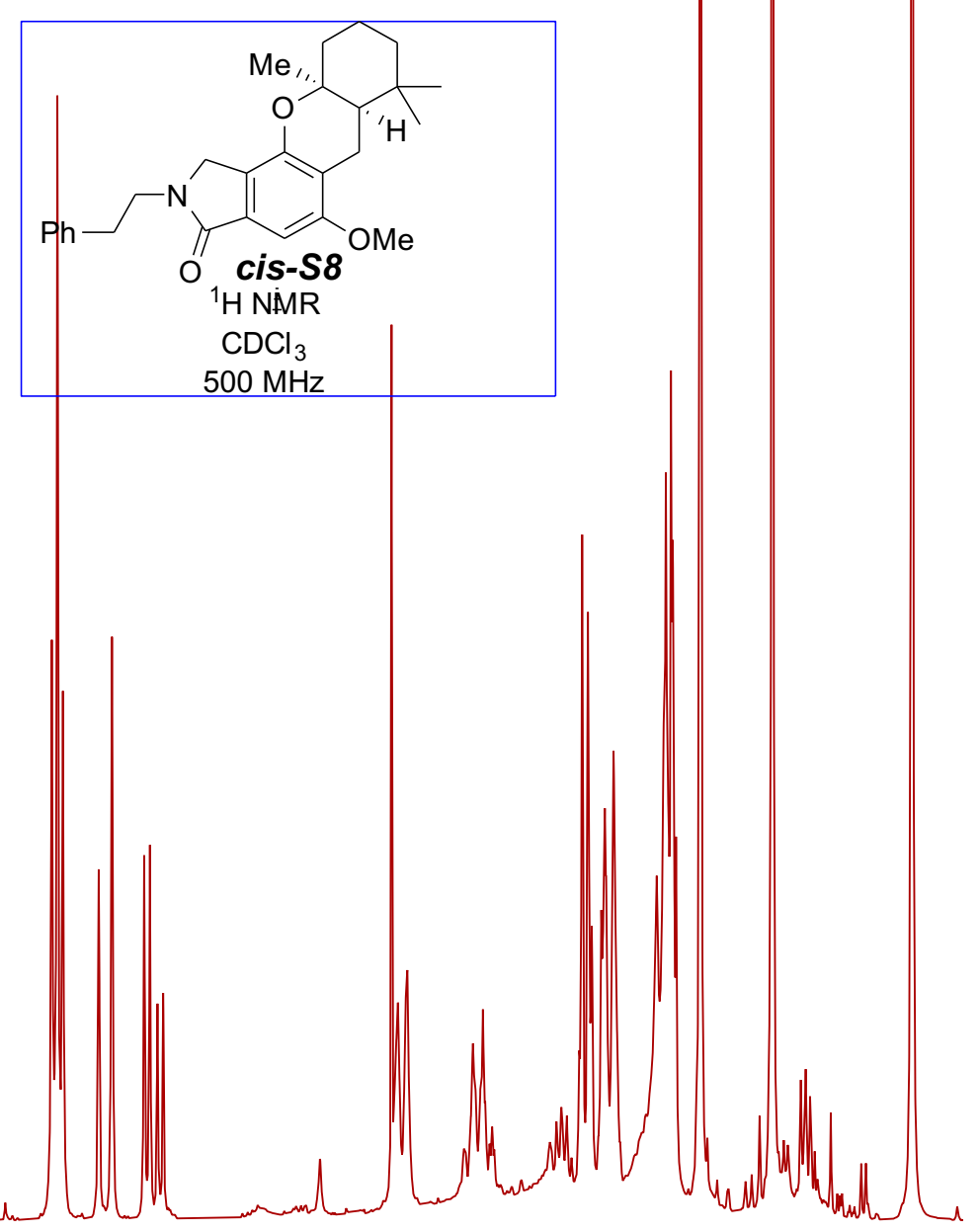

\begin{tabular}{|c|c|c|c|c|c|}
\hline 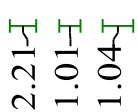 & & $\stackrel{g}{m} \stackrel{9}{9}$ & 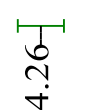 & 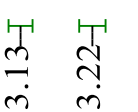 & $\begin{array}{l}T \\
\text { ลे } \\
\text { d. }\end{array}$ \\
\hline 3.0 & 2.5 & 2.0 & 1.5 & $115^{\circ}$ & 0. \\
\hline
\end{tabular}




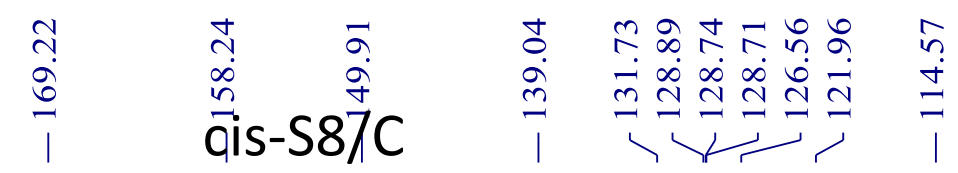

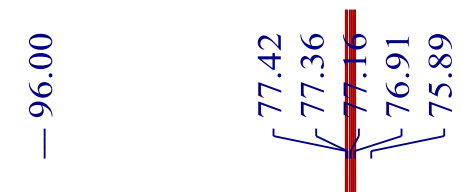

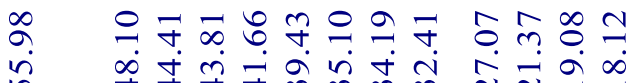

n

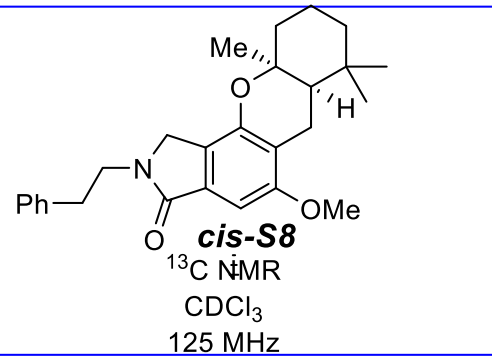




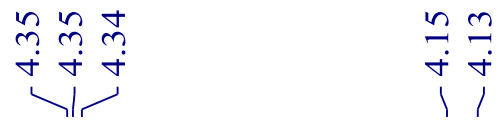

erinacerin $\mathrm{A}(2) / \mathrm{H}$

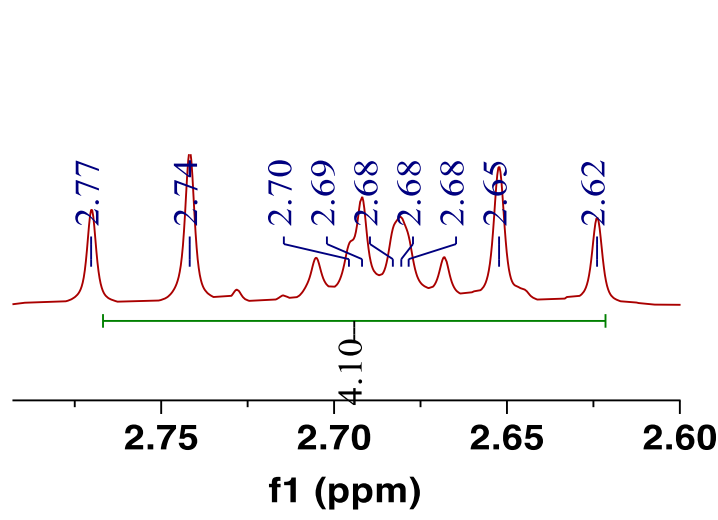

\section{erinacerin $A(2) / H$}

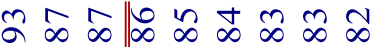

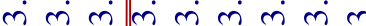

$1+\infty$
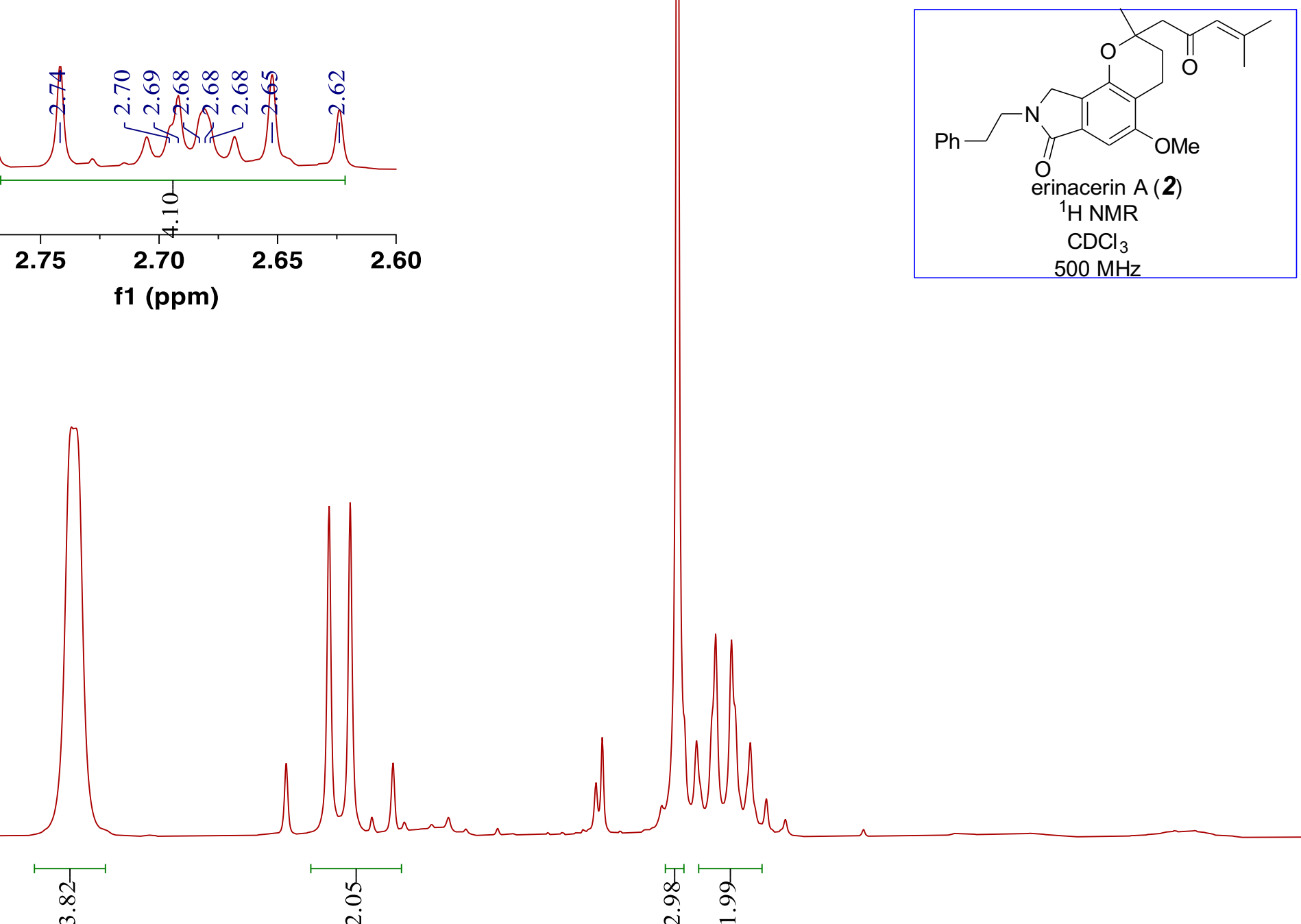

m
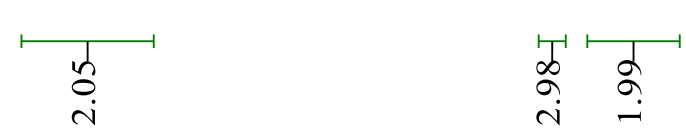

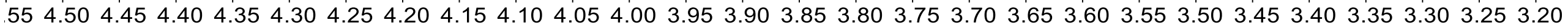
f1 (ppm) 


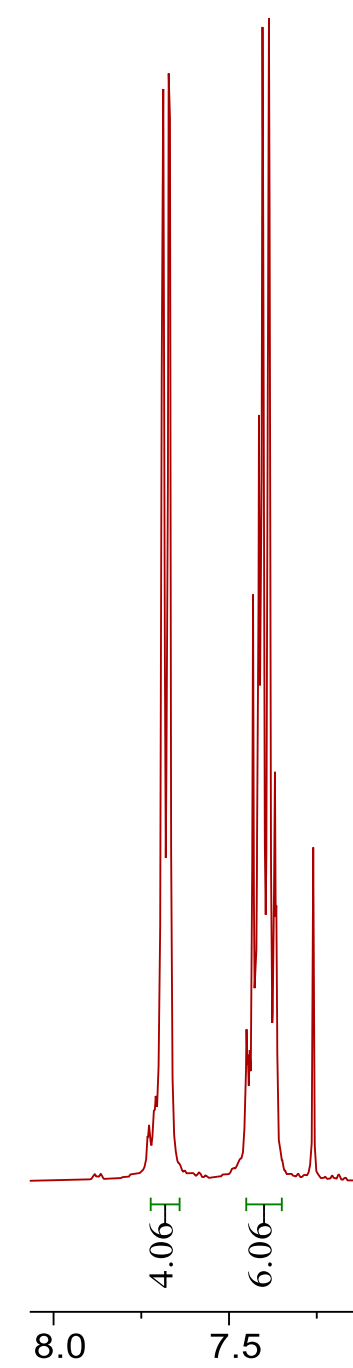

$\begin{array}{lll}7.0 & 6.5 & 6\end{array}$

5.5
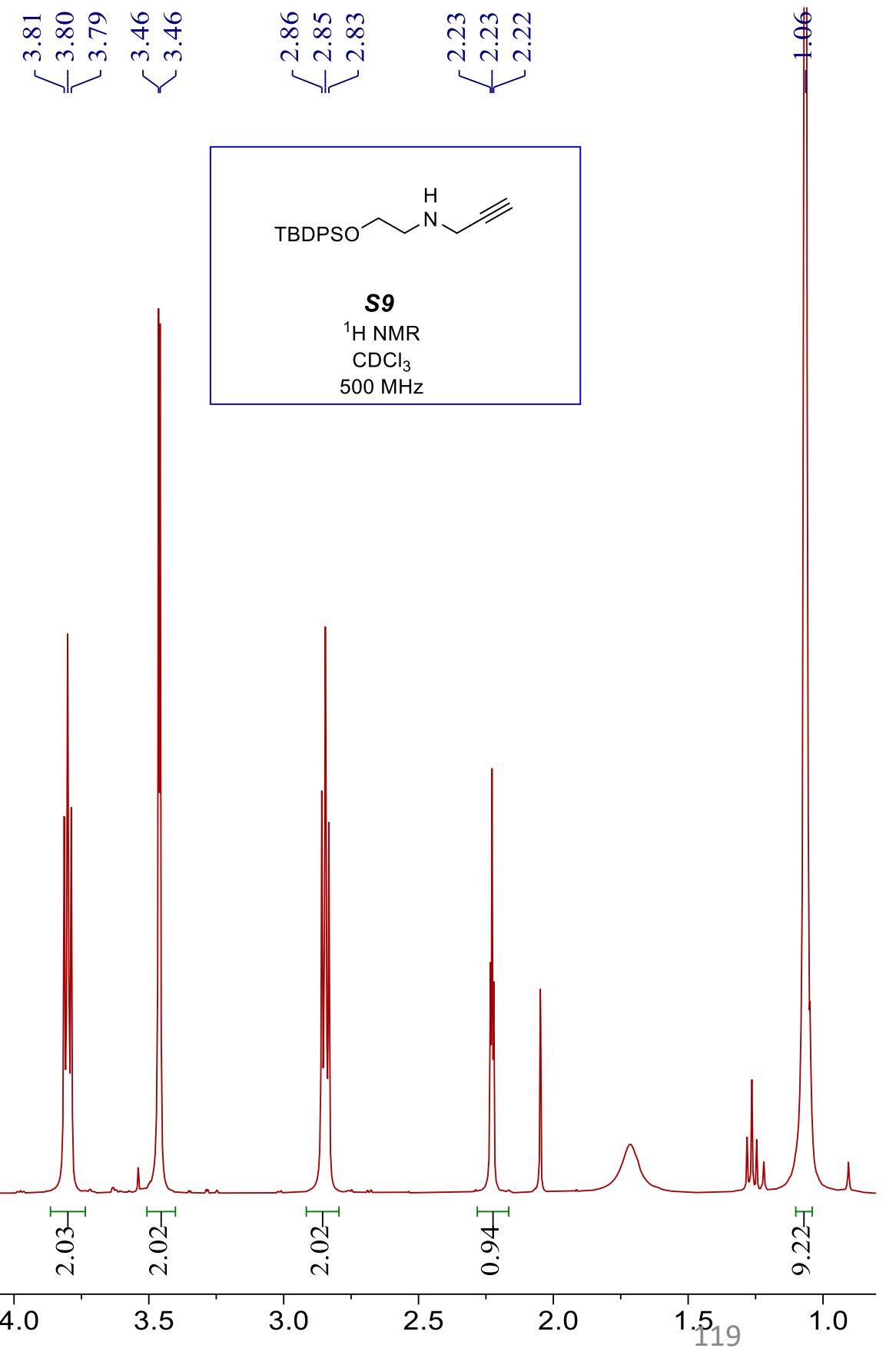
$\mathrm{S} 112$ of $\mathrm{S} 139$

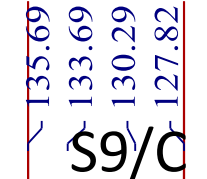

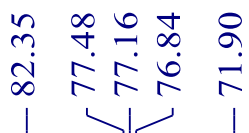

2
$\infty$
1
1

ọ

$\begin{array}{ll}\infty & \wp \\ \stackrel{0}{0} & \infty \\ & \end{array}$
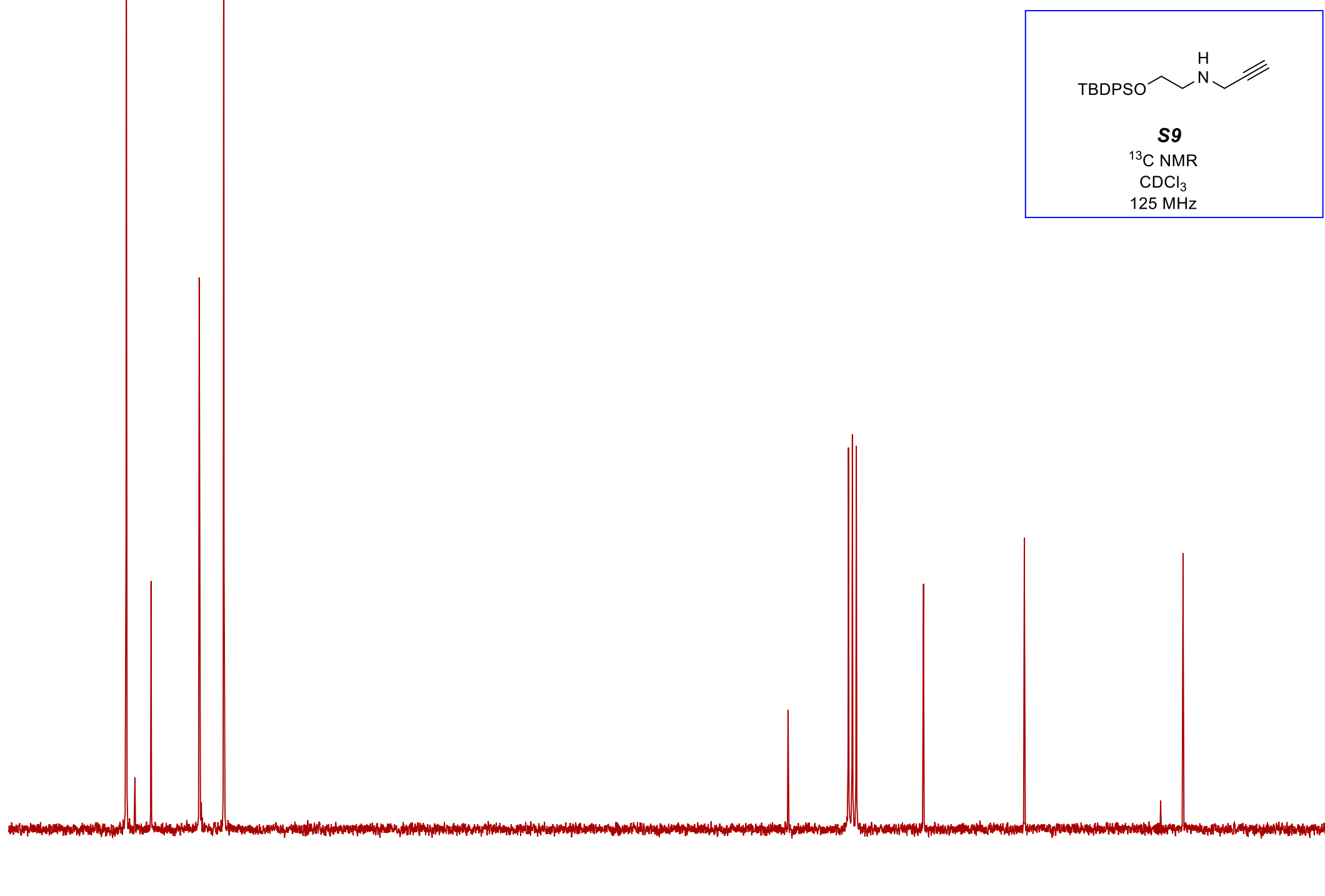

$$
45
$$

$\begin{array}{lll}140 & 135 & 130\end{array}$

$125 \quad 12$

$\begin{array}{llll}115 & 110 & 105 & 100\end{array}$

95

90

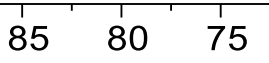

f1 (ppm)

65

60

55

50
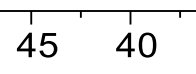

35

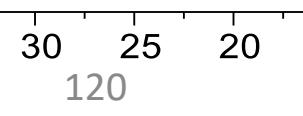



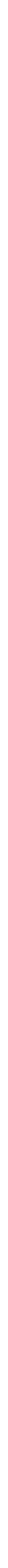


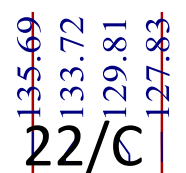

$\stackrel{n}{0}$
$\stackrel{0}{1}$

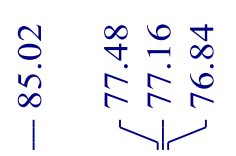

in

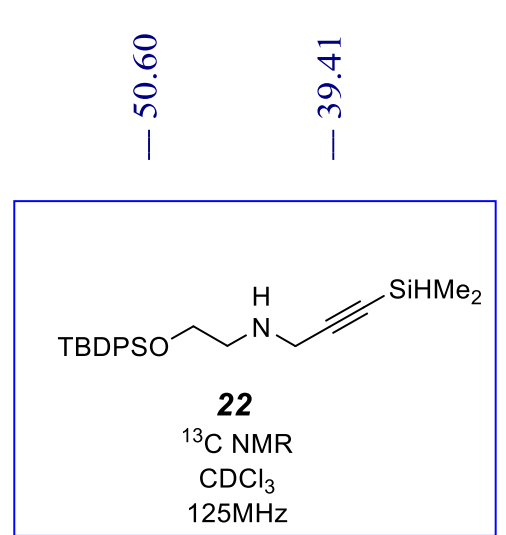

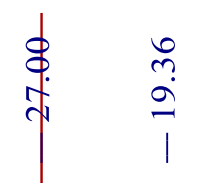



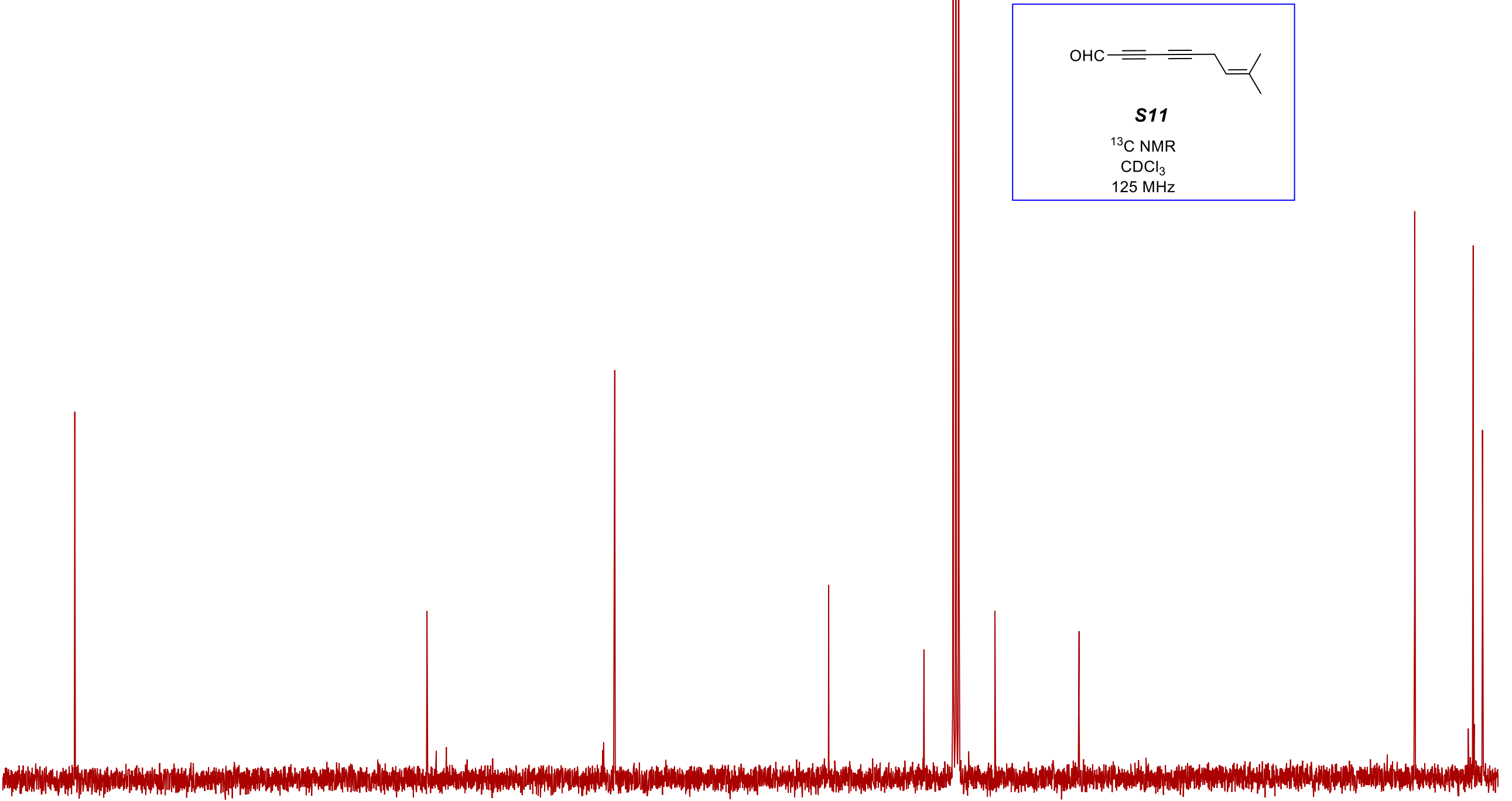

\begin{tabular}{|c|c|c|c|c|c|c|c|c|c|c|c|c|c|c|c|c|}
\hline 180 & 170 & 160 & 150 & 140 & 130 & 120 & 110 & $\begin{array}{c}100 \\
\mathrm{f} 1(\mathrm{ppm})\end{array}$ & 90 & 80 & 70 & 60 & 50 & 40 & 139 & 20 \\
\hline
\end{tabular}



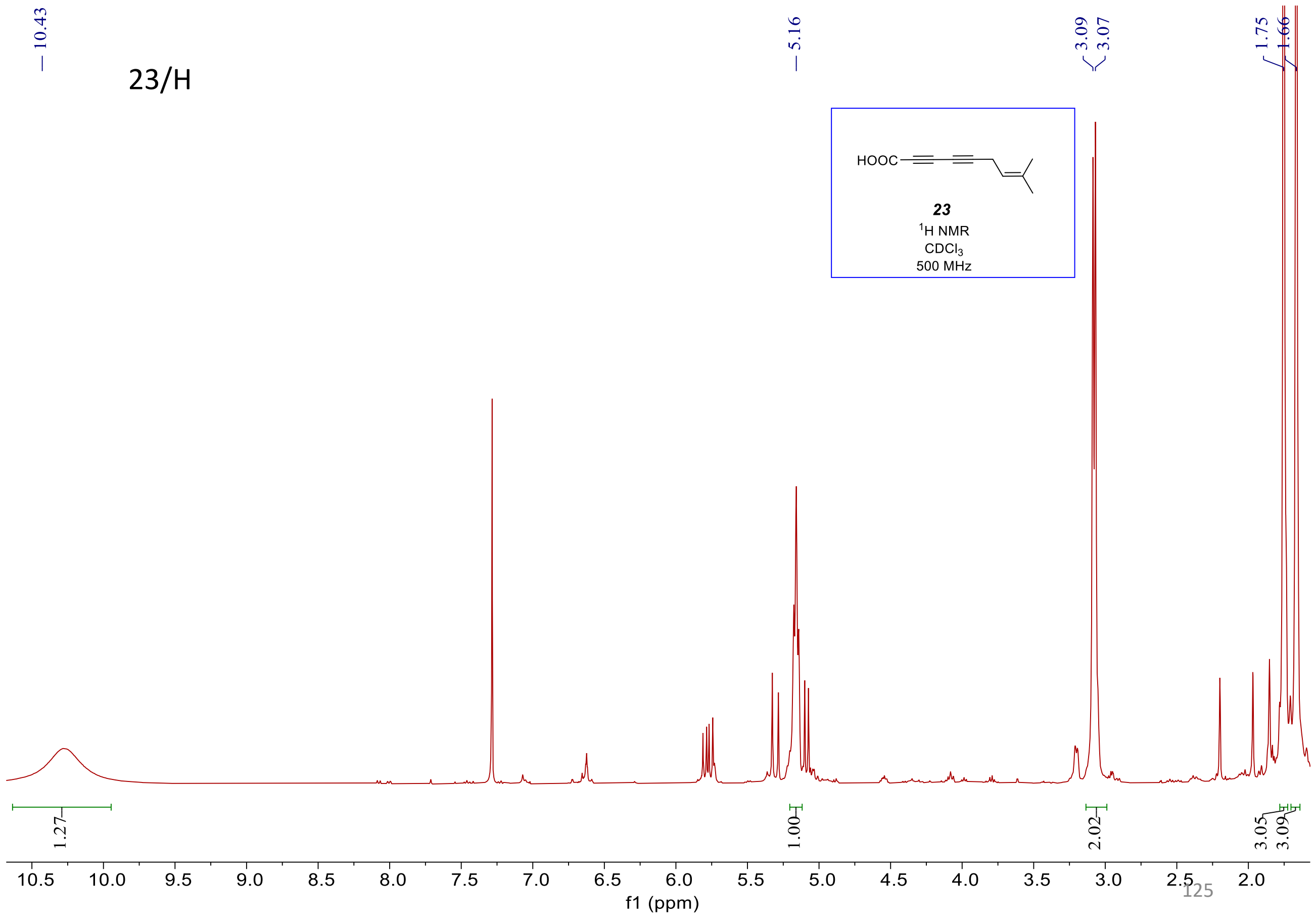


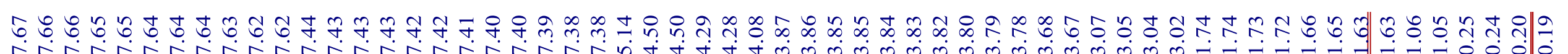

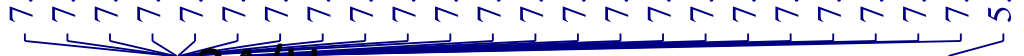

\section{समाम}

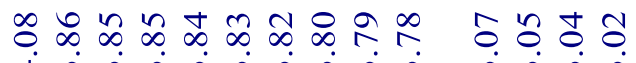

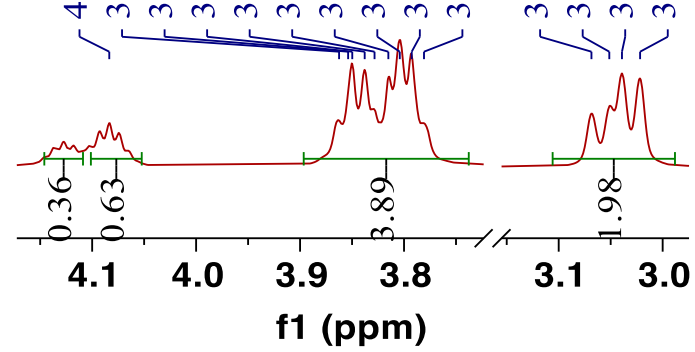

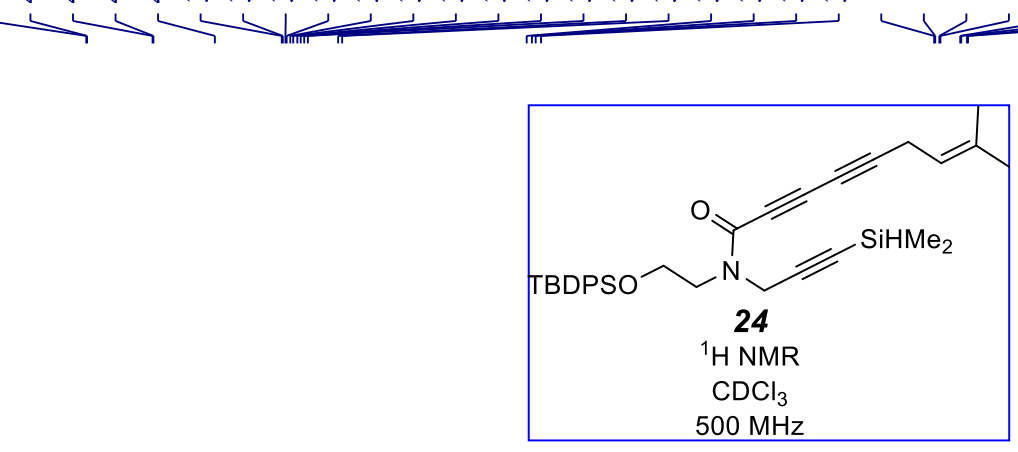
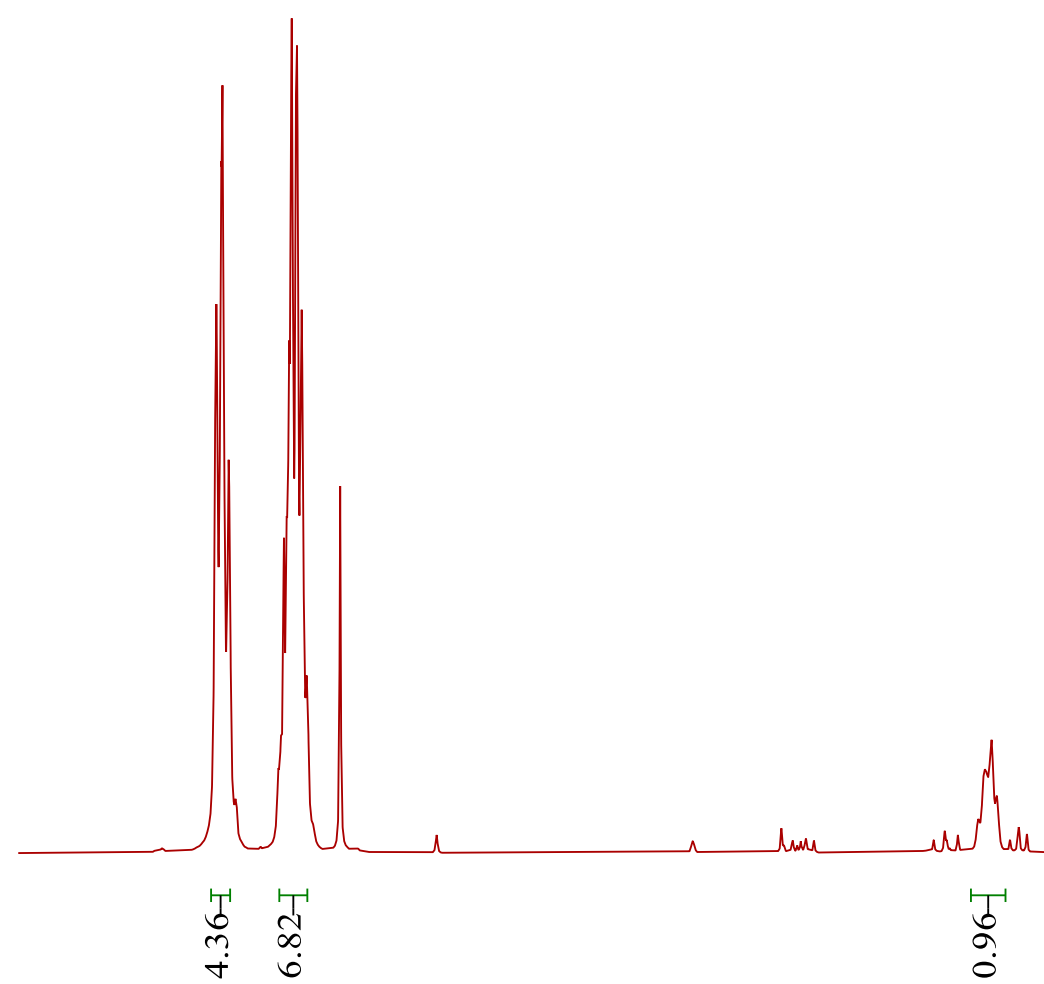
7.5

7.0

6.5

$6.0 \quad 5.5$

5.0

$$
\mathrm{f} 1(\mathrm{ppm})
$$

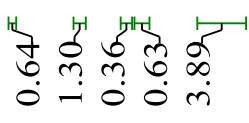

4.0

3.5

3.5

2.5

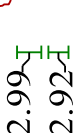

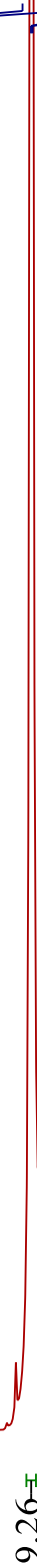

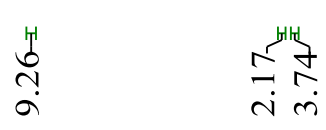

(ppm) $1.0126^{0.5}$ 
กิ ñn $\sqrt{2}$ 24र्टे
글

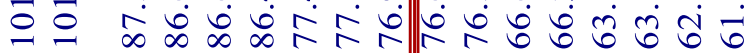

넉 守守 में

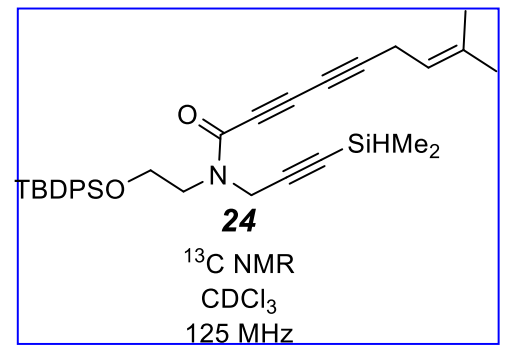

|

$150 \quad 140 \quad 130$

130

120

110

100

90

80
$\mathrm{f} 1(\mathrm{ppm})$

70

60

50

40

30

20

10

0 


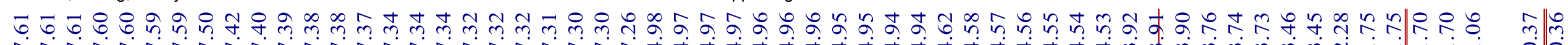

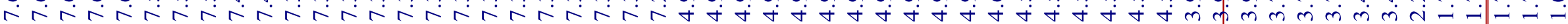
$26 a / H$
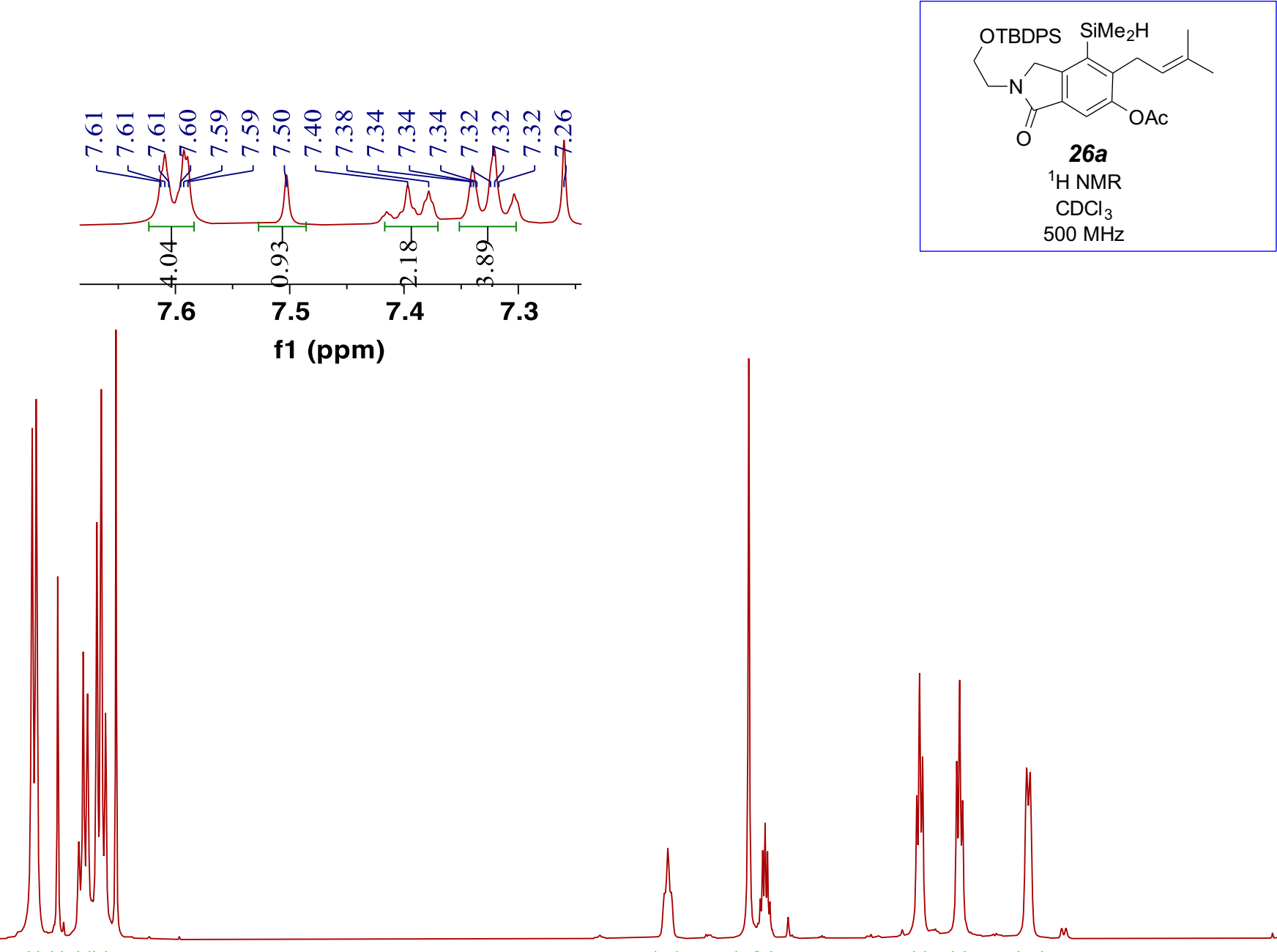

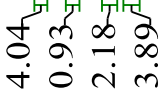

7.5

7.0

6.5

5.5

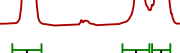

蒾
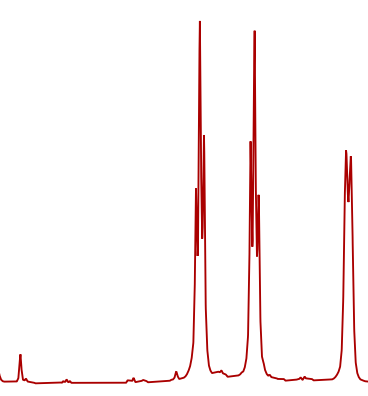

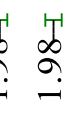

4.5

f1 (ppm)

$3.5 \quad 3.0 \quad 2.5$

à

ลूँ 

in $\infty$
จิ

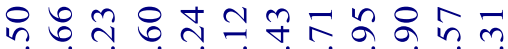

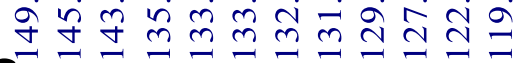
26a/C

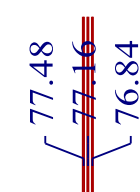

ธำ

किं कि

$\stackrel{n}{\vec{n}}$

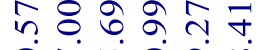

세요

$<<1<1<$
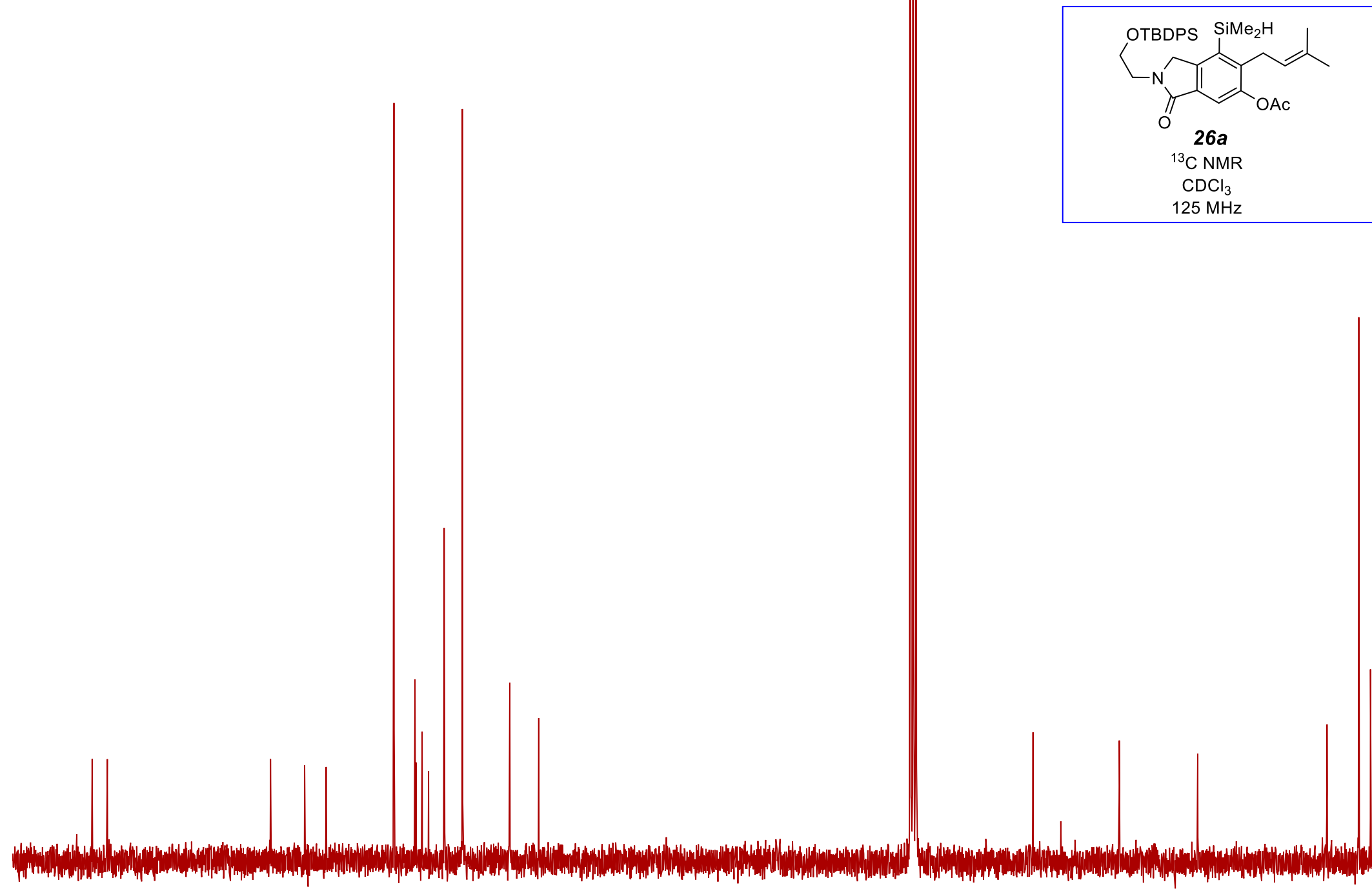


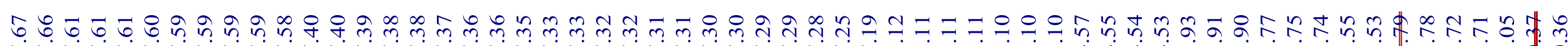

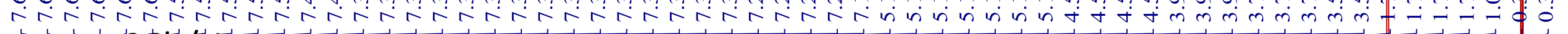
201010

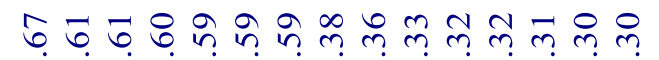
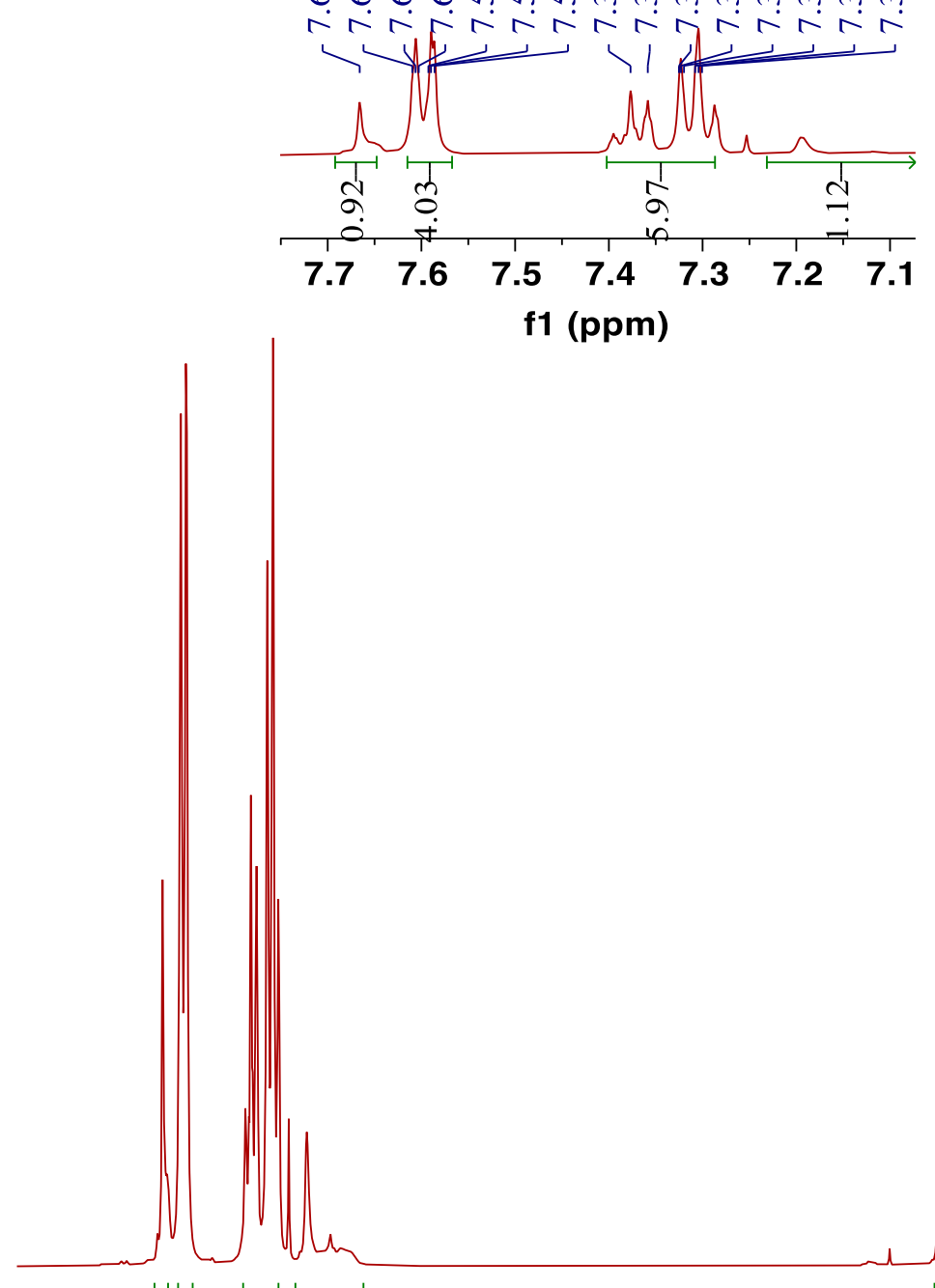

स्स तूํำ
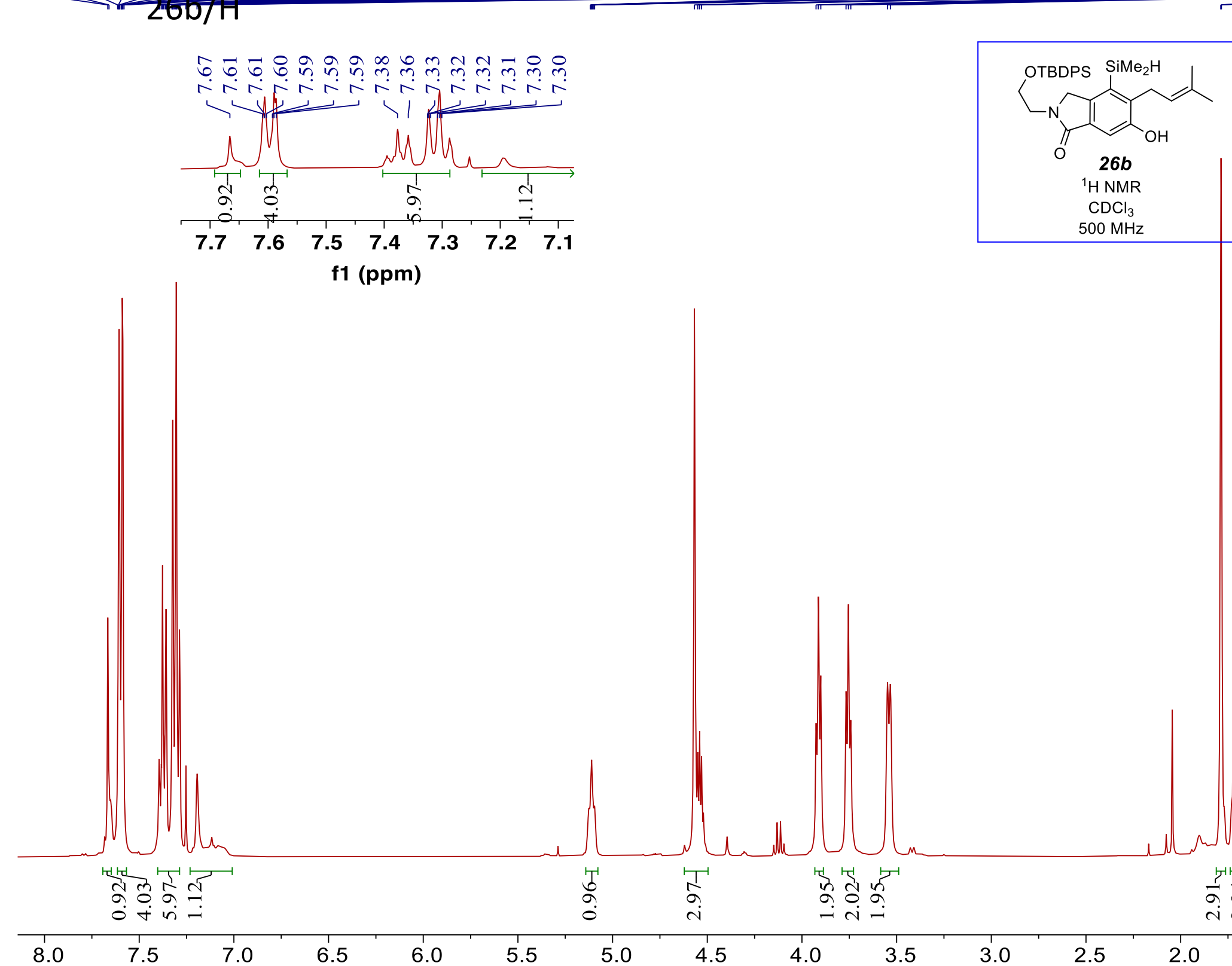

6.5
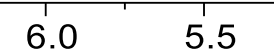

$5.0 \quad 4.5$

f1 (ppm)
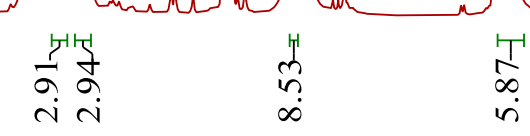

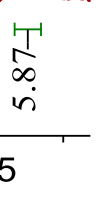



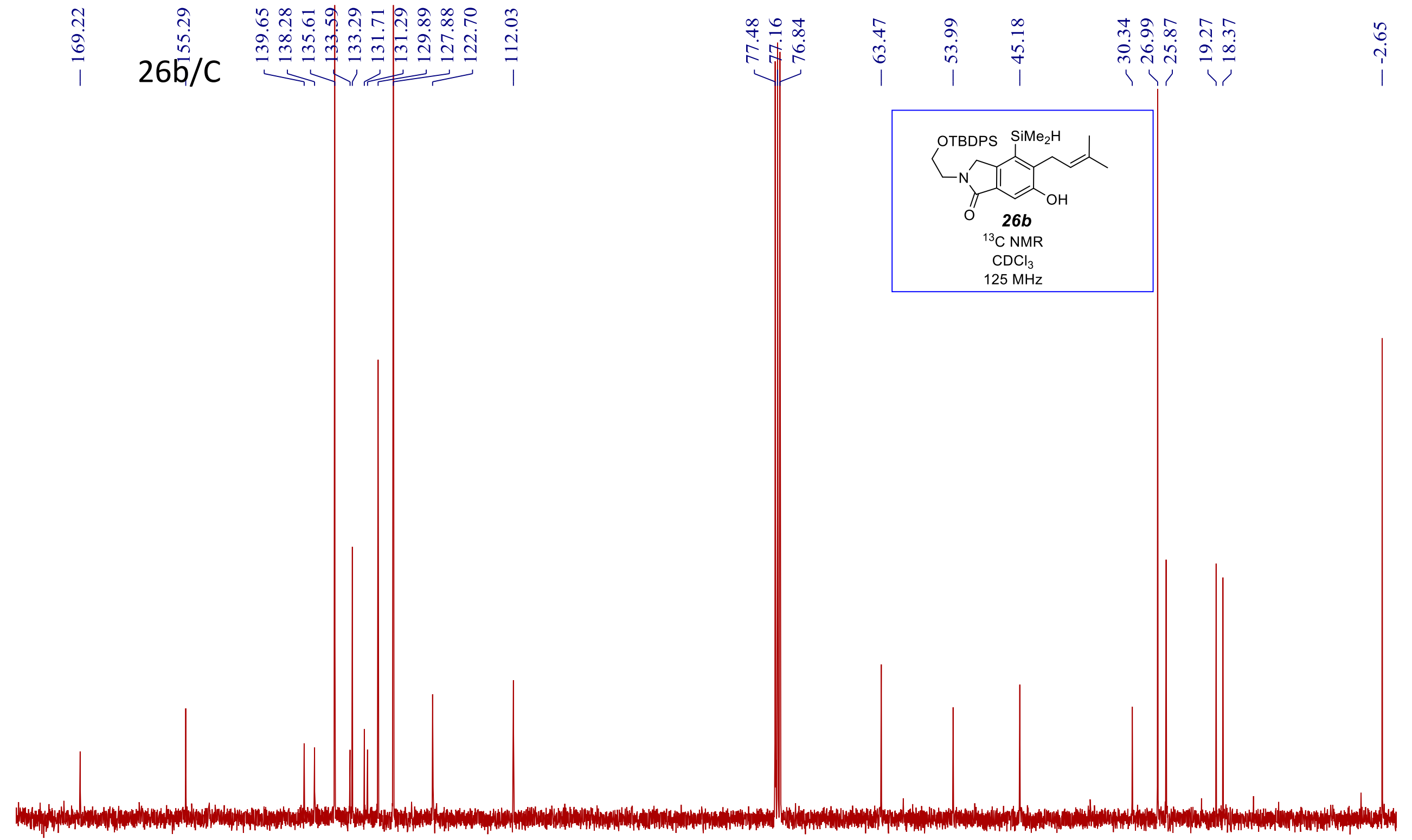


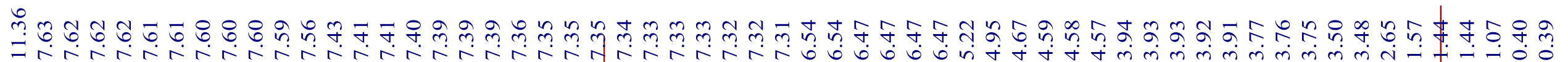
$27 \mathrm{a} / \mathrm{H}$

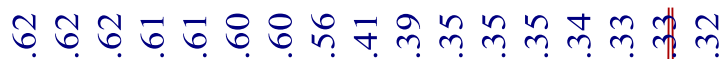

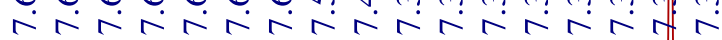
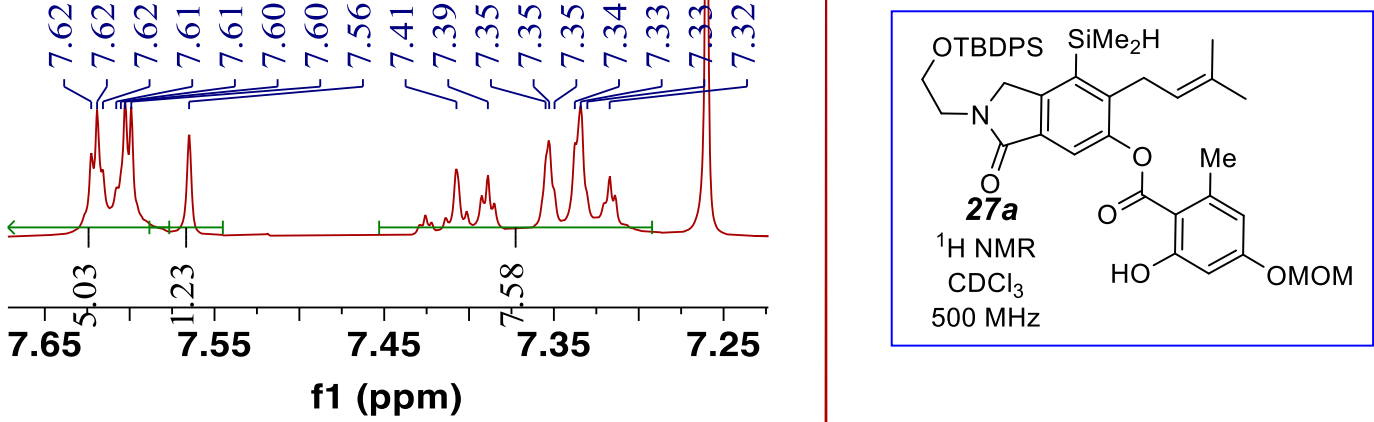

f1 (ppm)

\section{5} $500 \mathrm{MHz}$
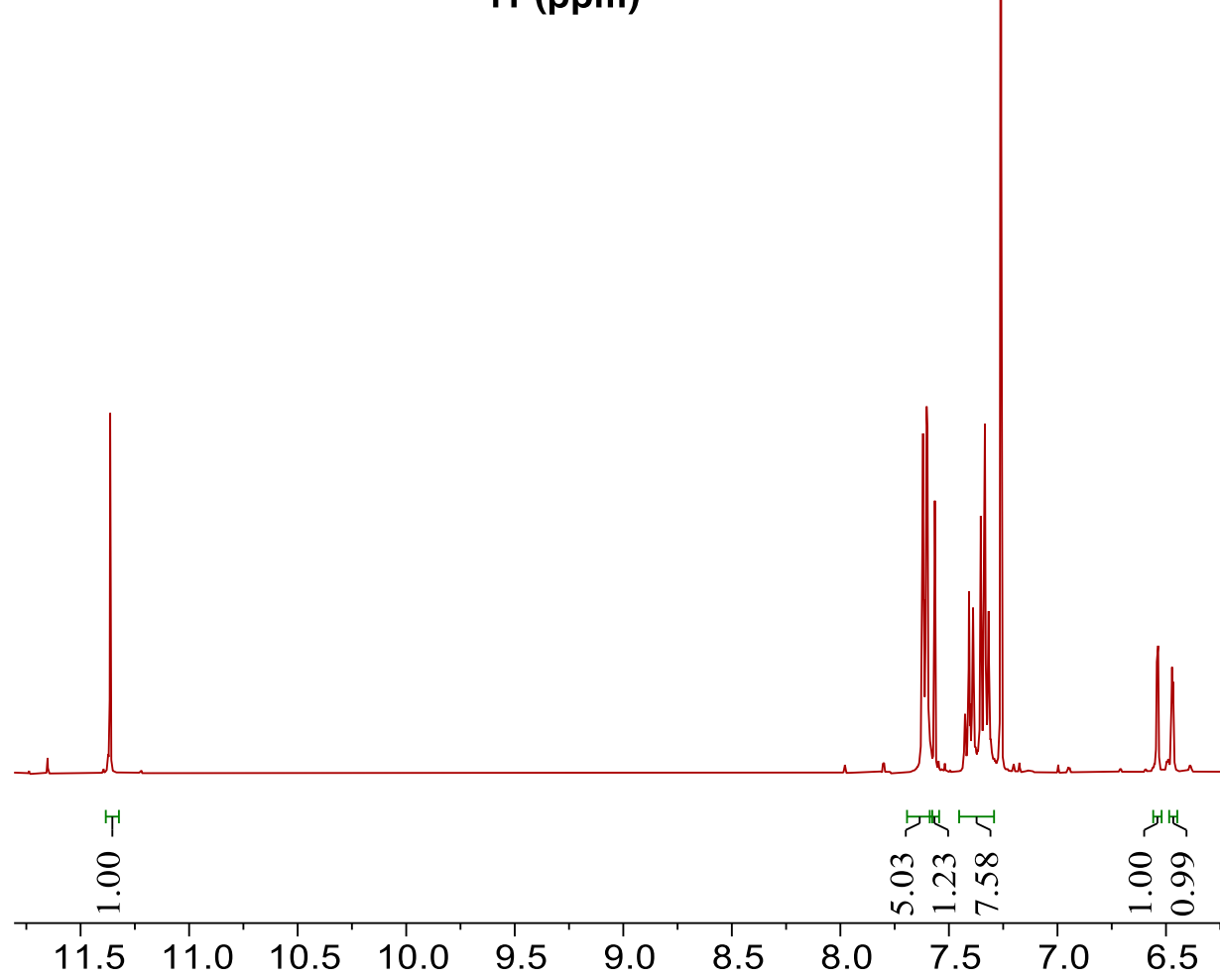

กิ่

8 बे

7.0
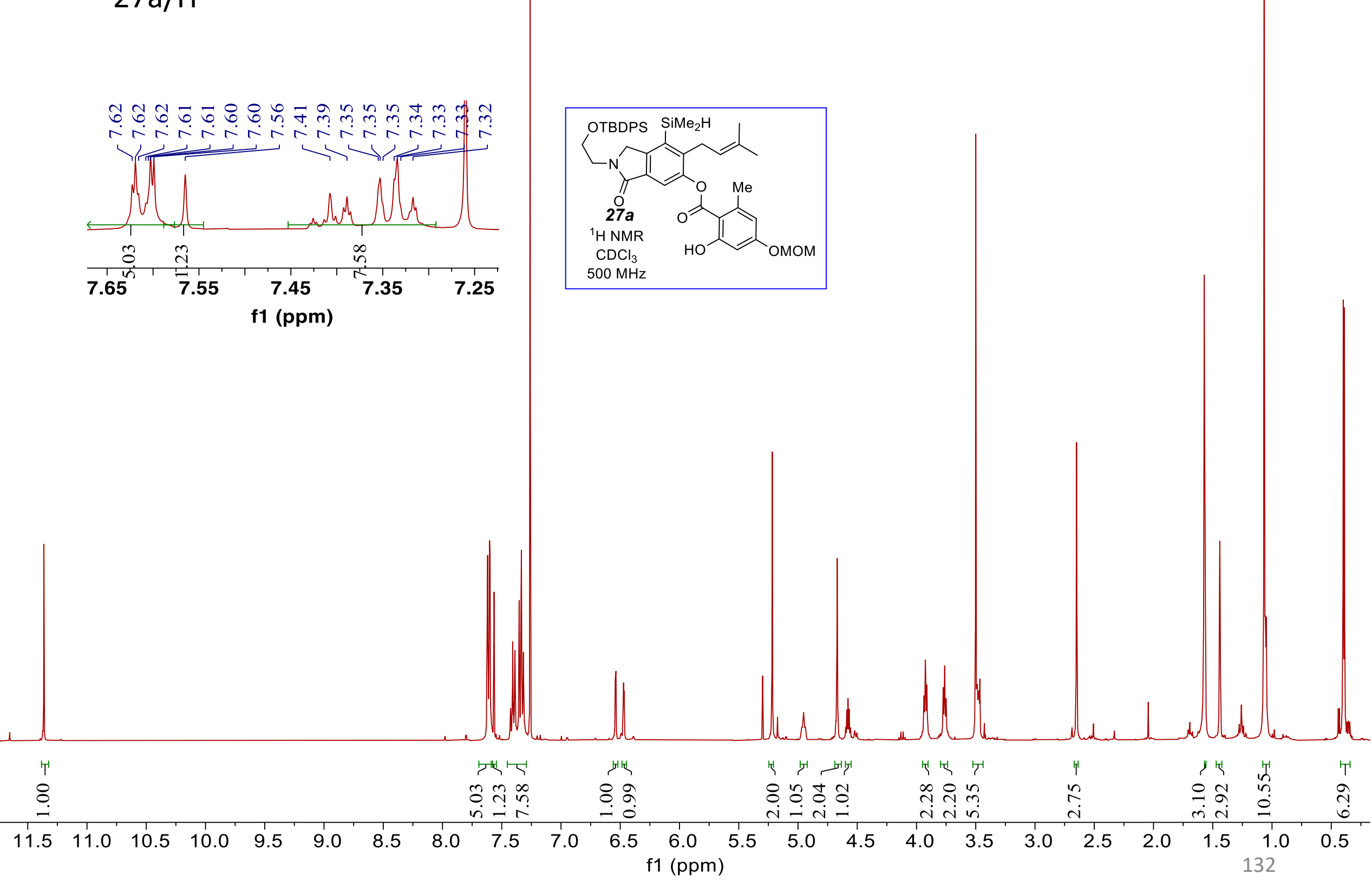

定




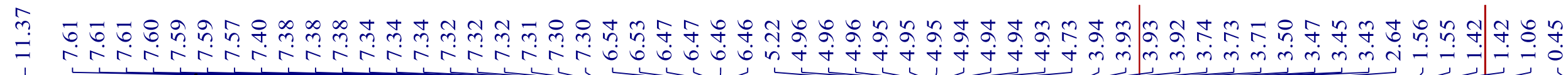

చִ

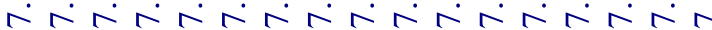

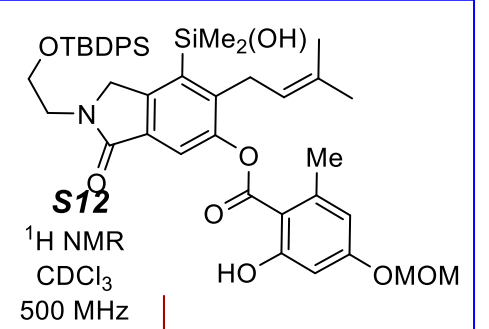

$500 \mathrm{MHz}$
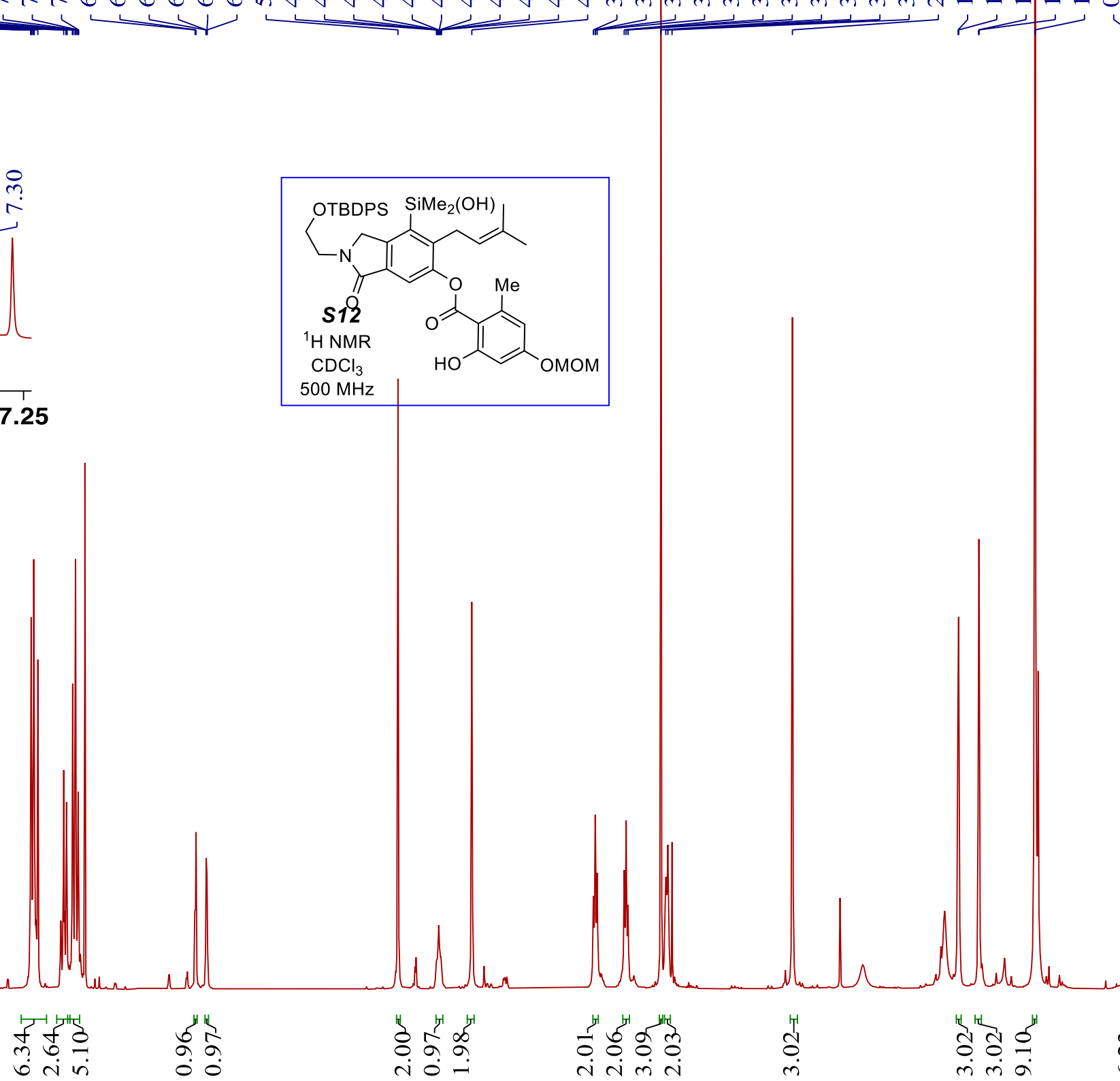

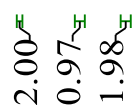

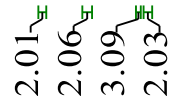

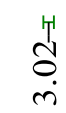




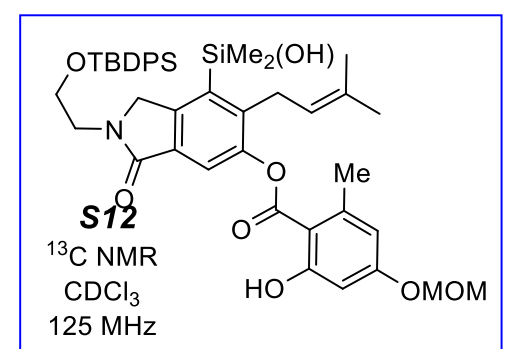


กิ่ สุิ ๆ.

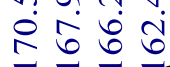

$11, \frac{1}{1}, 29 / C$

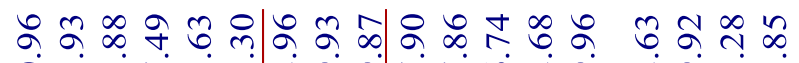

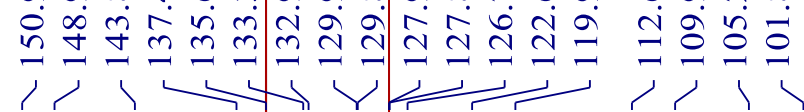

\section{ô}

$\begin{array}{ll}a & n \\ 0 & n \\ 0 & n \\ 1 & 1\end{array}$

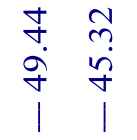

$\overrightarrow{0} \infty \infty \infty$ ํ. กิ $\mathrm{S}^{1}$

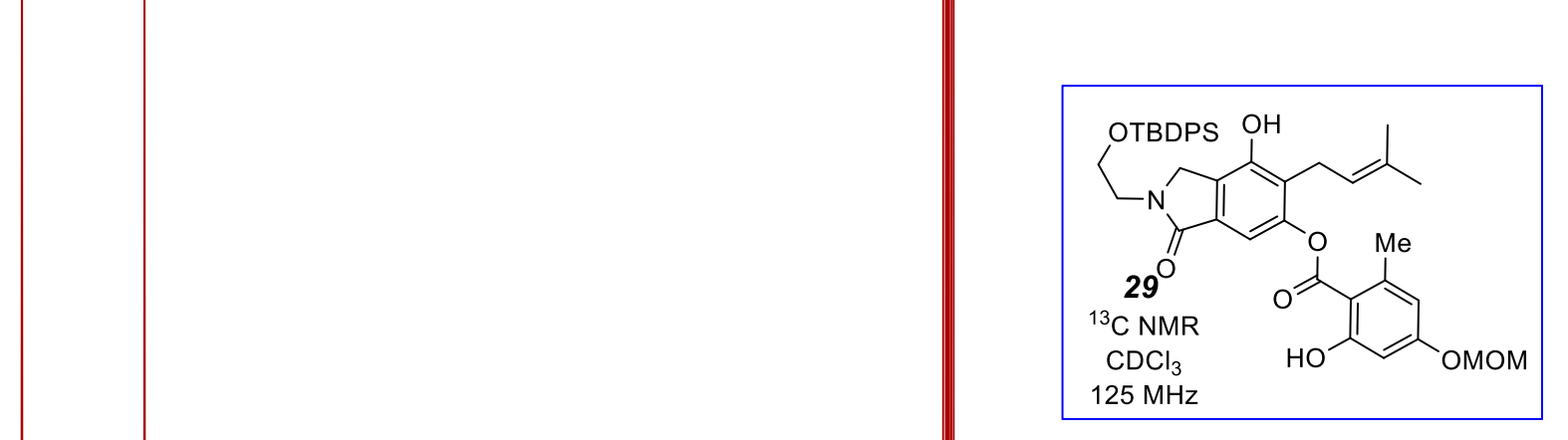



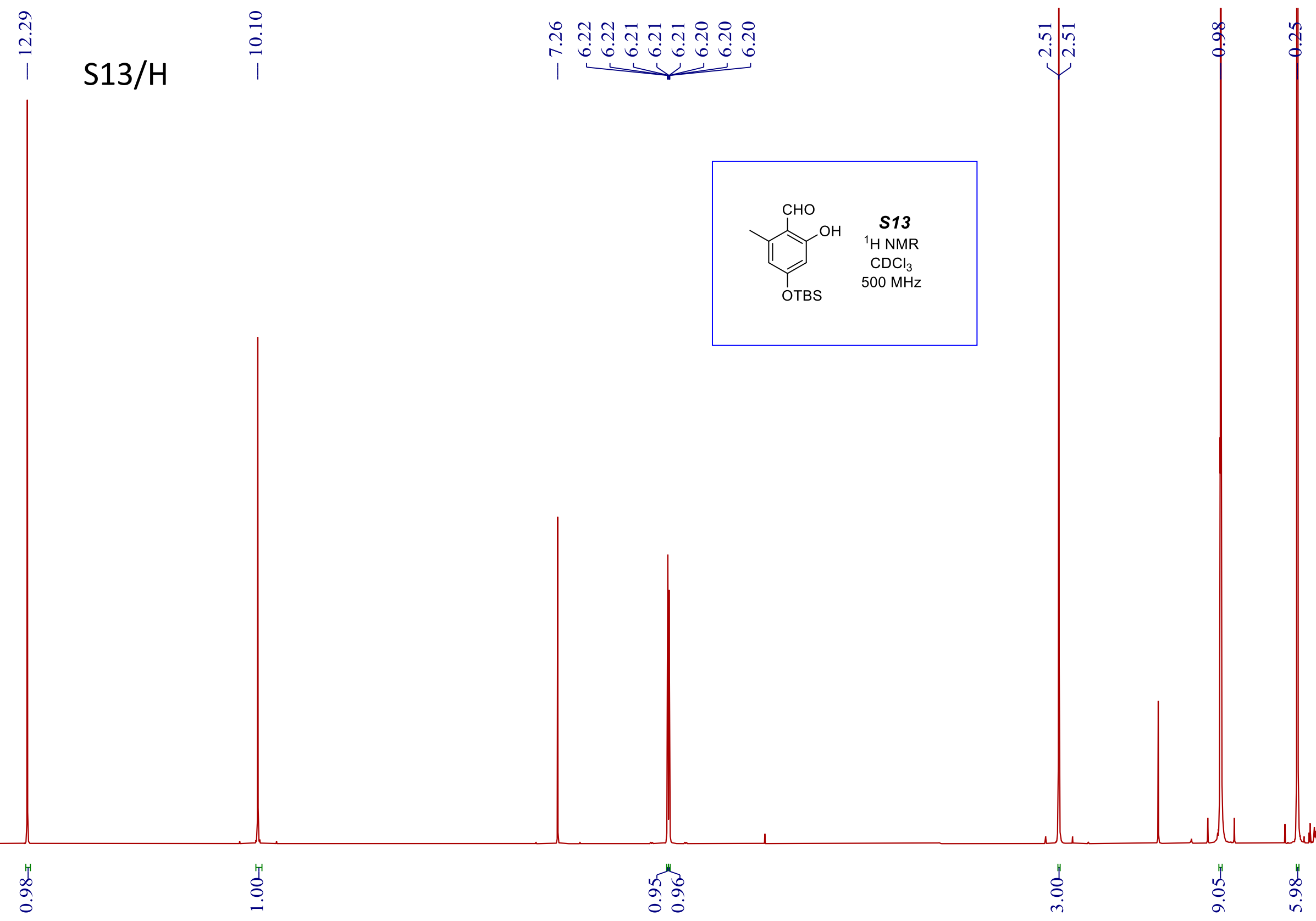

$\begin{array}{llllllllllllllllllllllllllll}12.5 & 12.0 & 11.5 & 11.0 & 10.5 & 10.0 & 9.5 & 9.0 & 8.5 & 8.0 & 7.5 & 7.0 & 6.5 & 6.0 & 5.5 & 5.0 & 4.5 & 4.0 & 3.5 & 3.0 & 2.5 & 2.0 & 1.5 & 1.0 & 138.5 & 0.0\end{array}$ 


S13/C

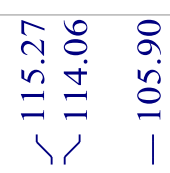

in m

1) $\infty$

$\sqrt{1}$
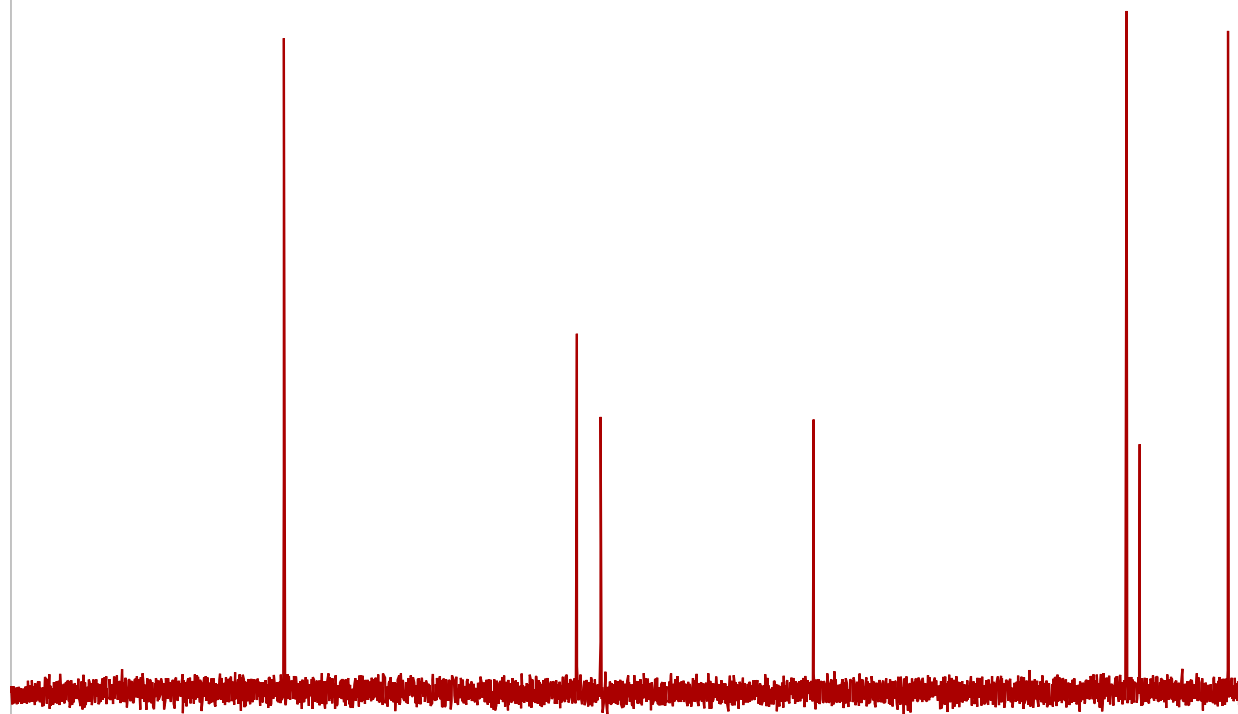

210200

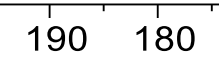

$170 \quad 160$

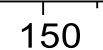

$140 \quad 130$

120

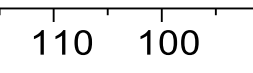

f1 (ppm)
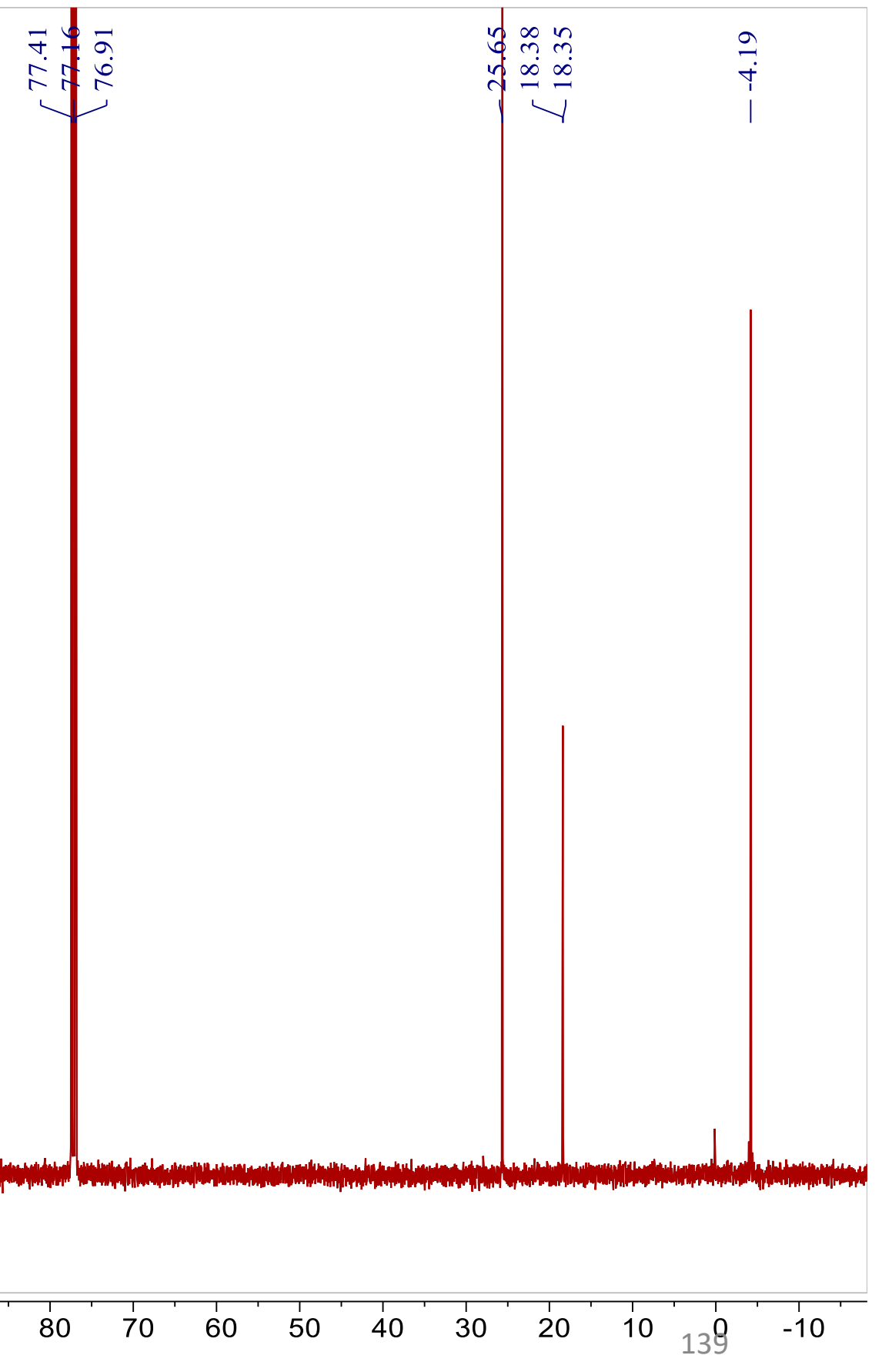


\section{$25 \mathrm{c} / \mathrm{H}$}
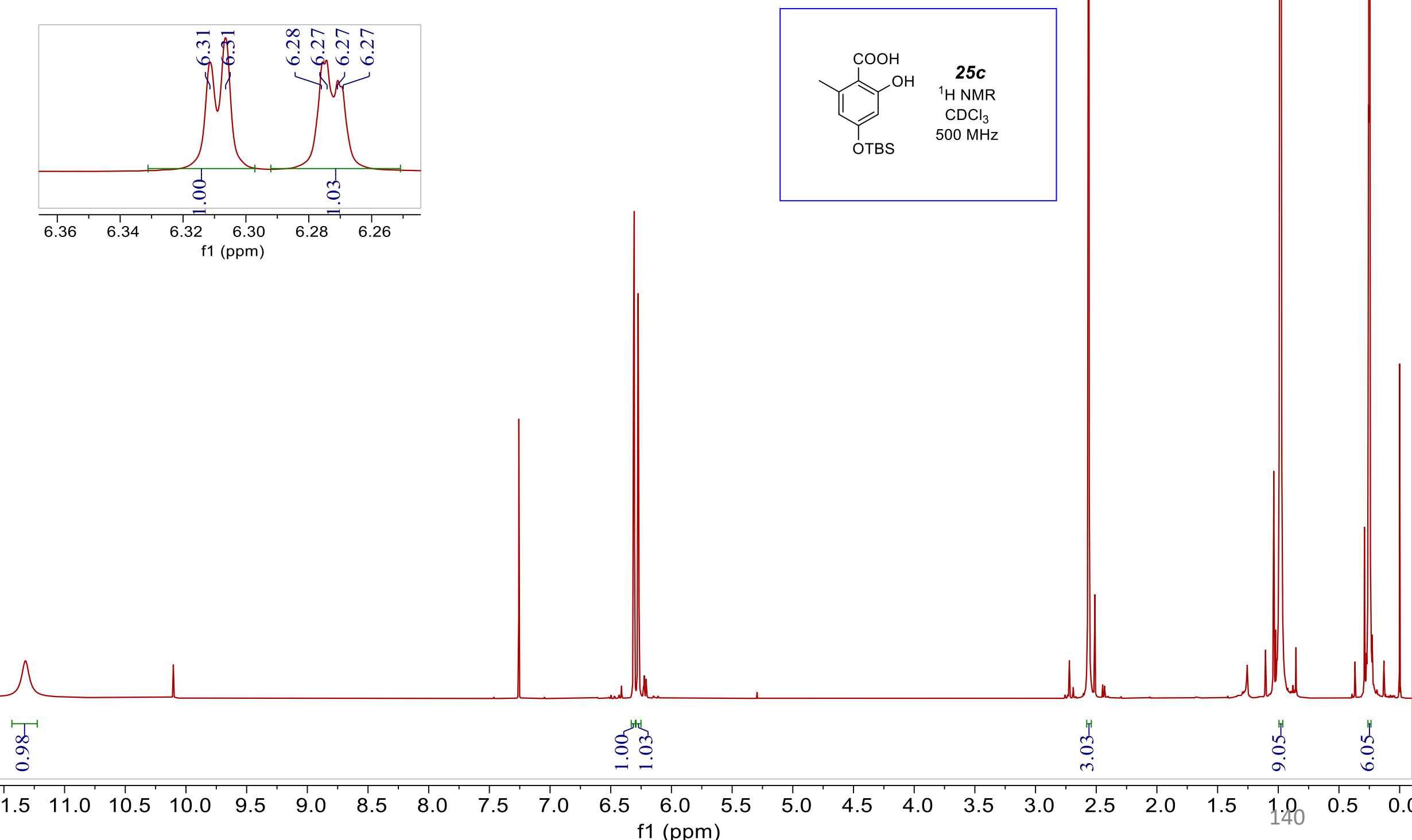
 \\ Supporting Information}

$\begin{array}{ll}\infty & 0 \\ 0 & 0 \\ 0 & 0 \\ 1 & 0 \\ 1 & 1\end{array}$

do n

ปั

숭요

计
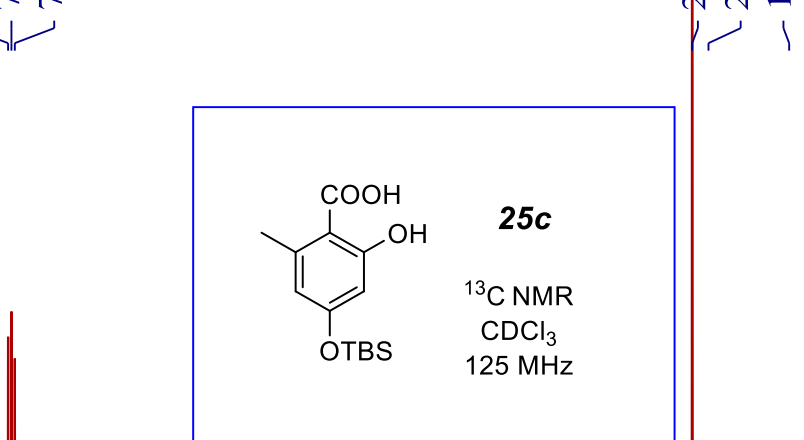

$25 c$

${ }^{13} \mathrm{C}$ NMR

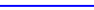


तुర

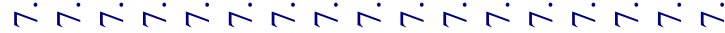

—

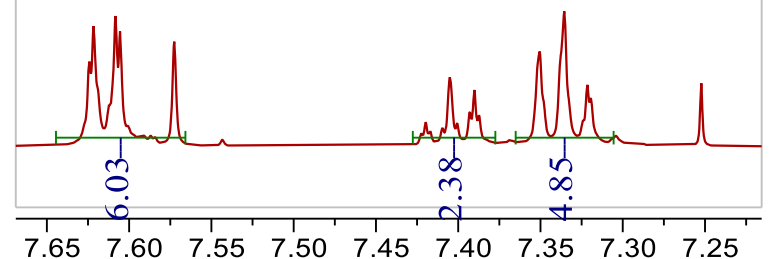

$\begin{array}{lllllllllll}7.65 & 7.60 & 7.55 & 7.50 & 7.45 & 7.40 & 7.35 & 7.30 & 7.25\end{array}$ f1 (ppm)
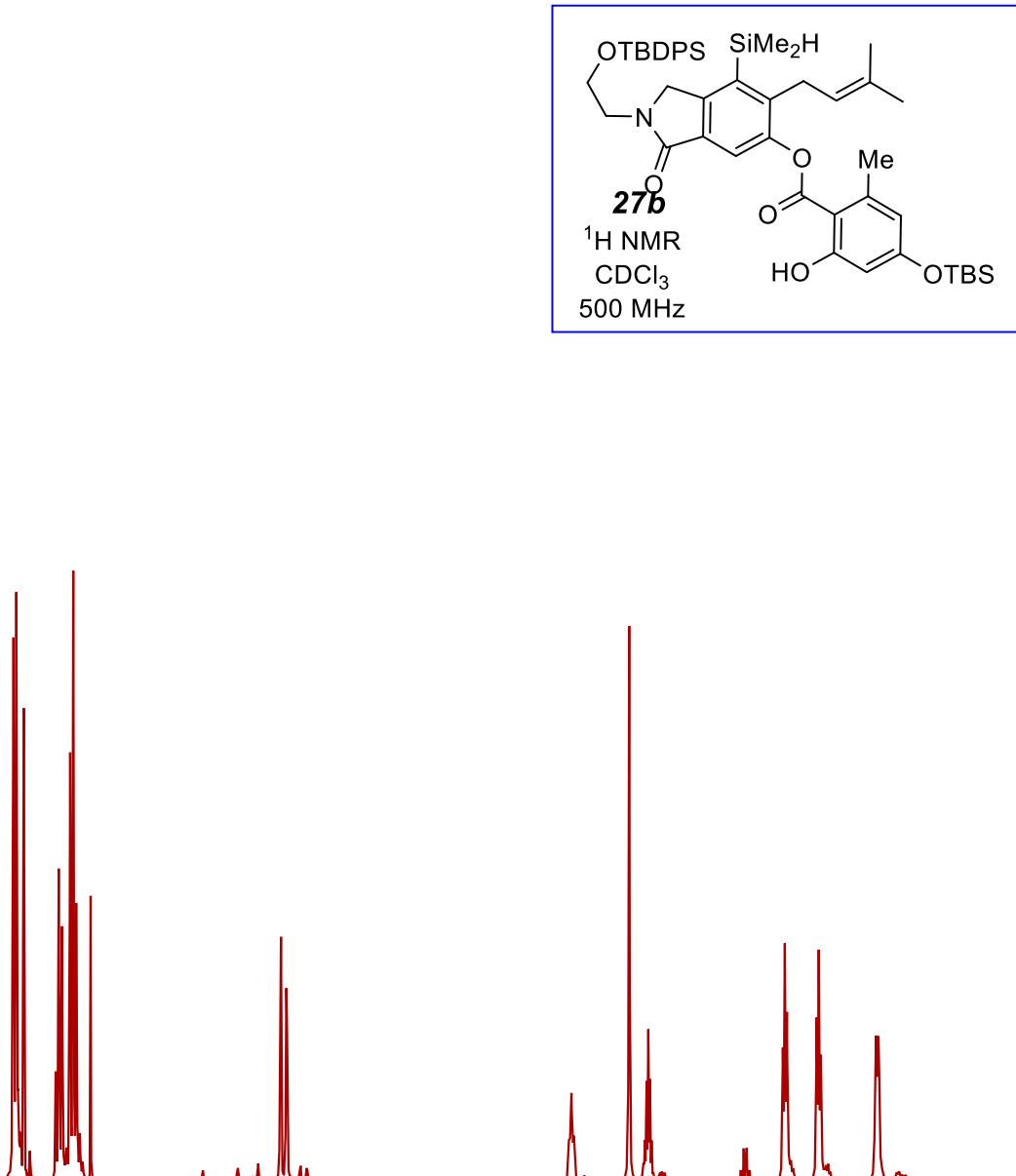

1

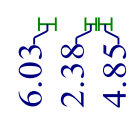

ह઼ํ.

年

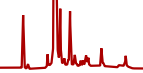

ô. $\begin{array}{cc}6.5 & 6.0 \\ \mathrm{f} 1 & (\mathrm{ppm})\end{array}$ 


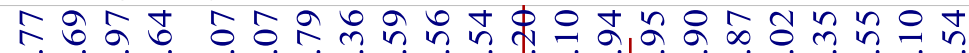

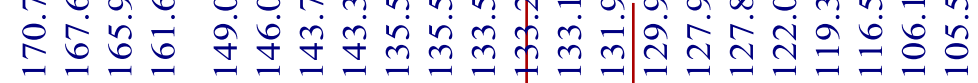

Supporting Information

S135 of S139

1) $27 \mathrm{~b} / \mathrm{C}$

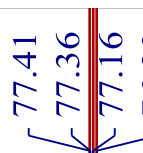

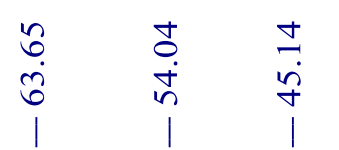

के

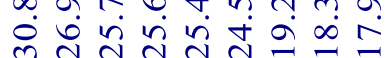

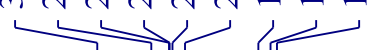

n

ì
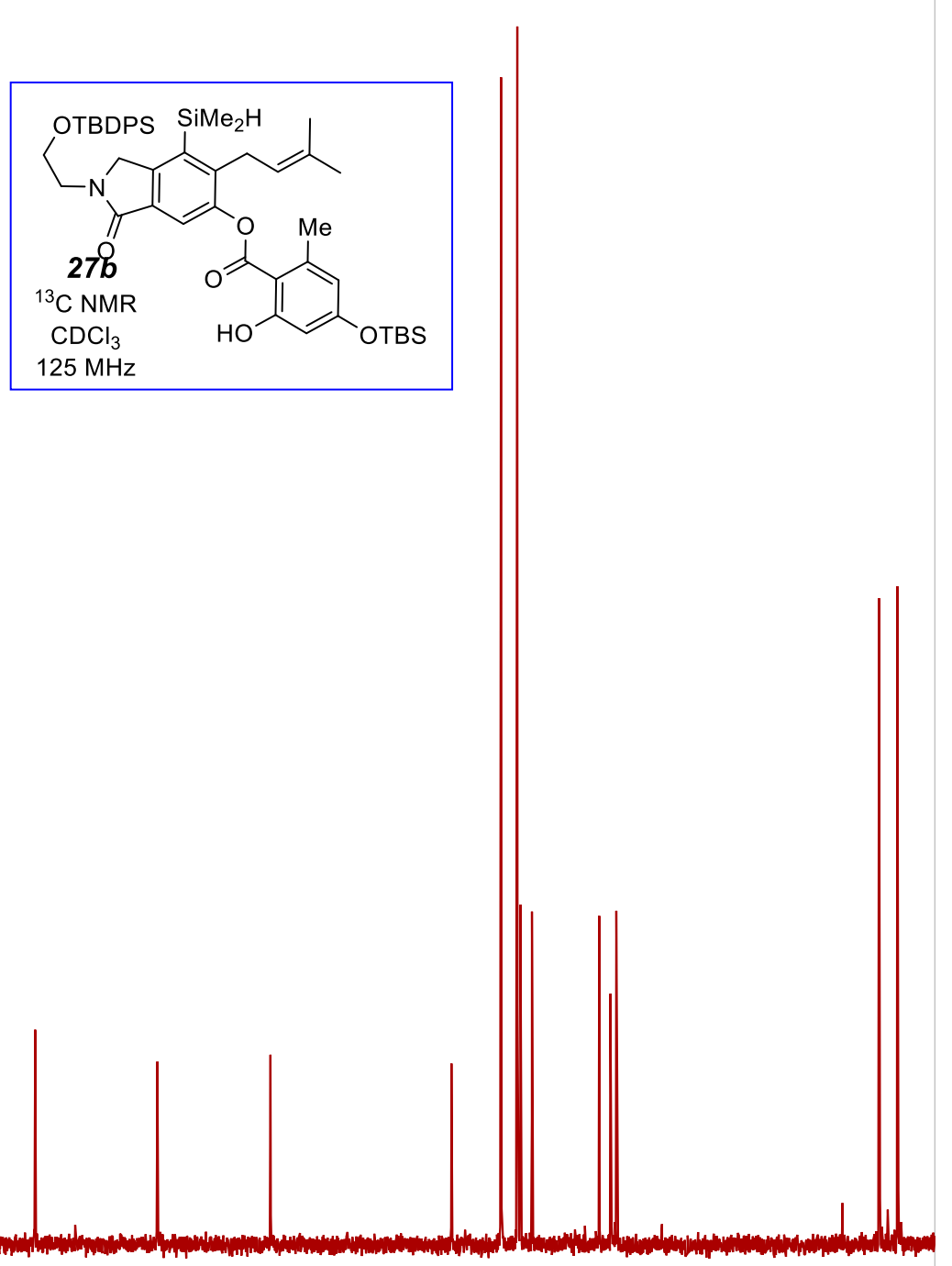

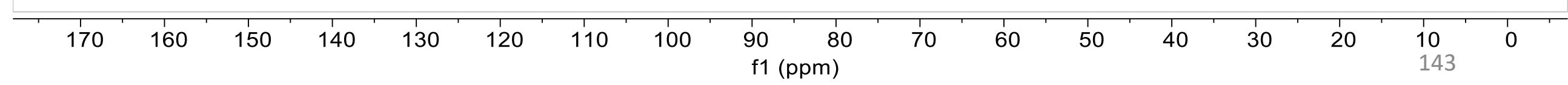


尚 $28 / \mathrm{H}$
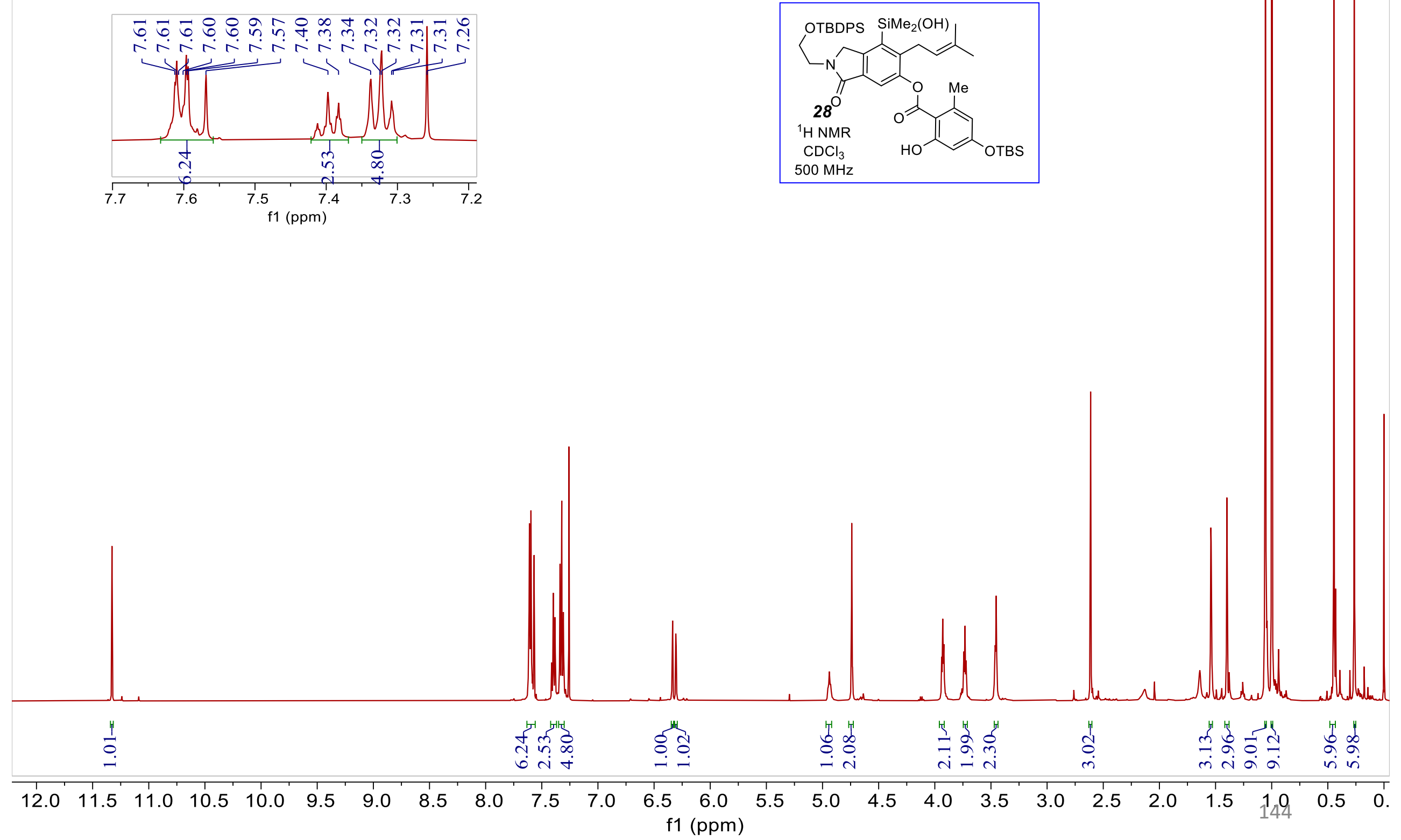


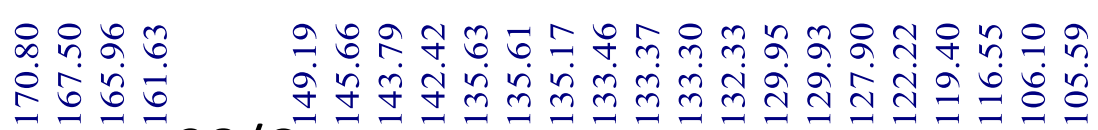

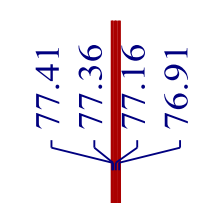

กิ

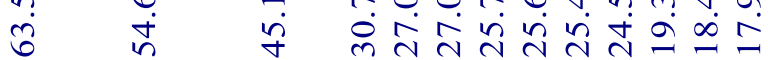
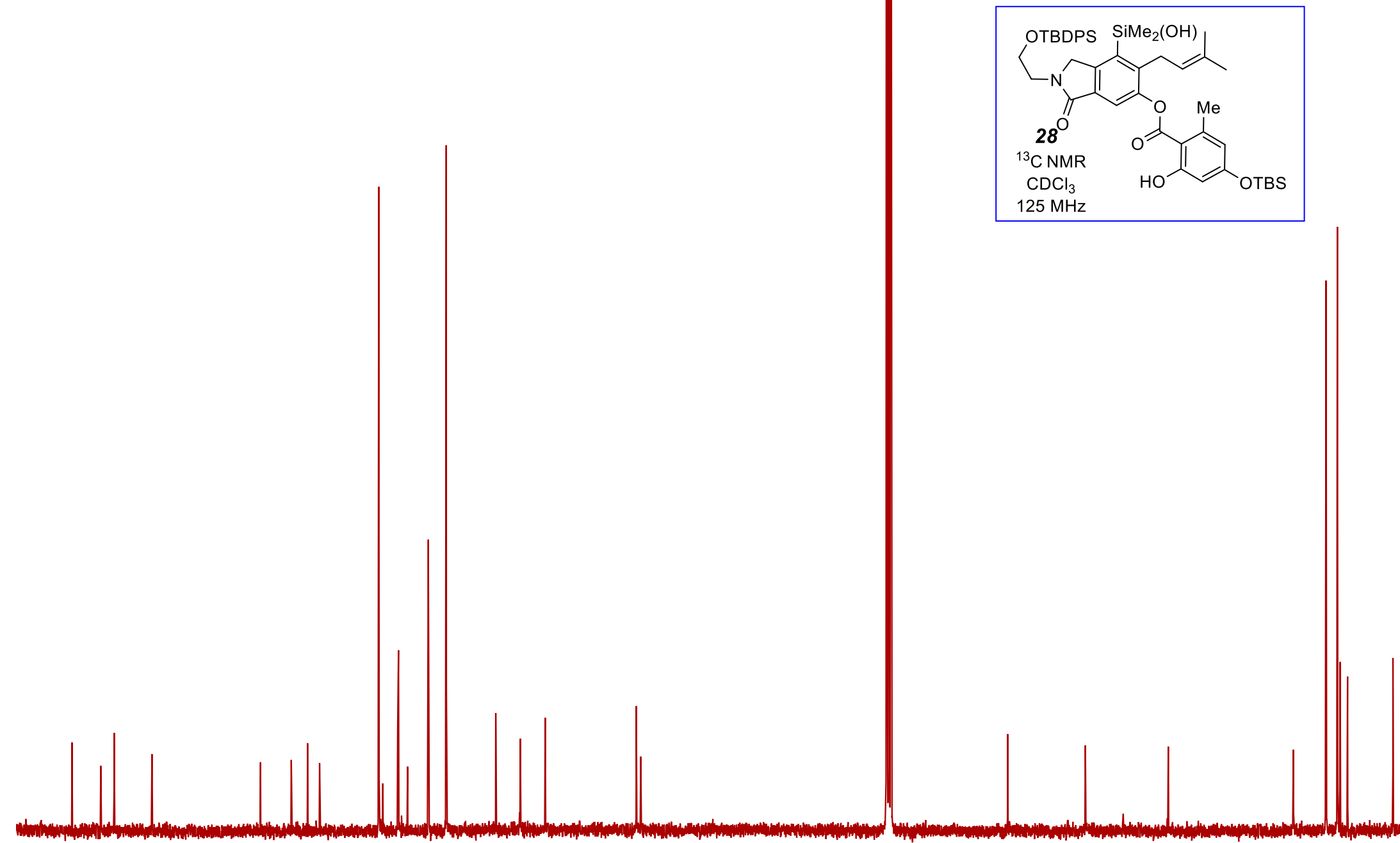

\begin{tabular}{|c|c|c|c|c|c|c|c|c|c|c|c|c|c|c|c|c|c|c|}
\hline 170 & 160 & 150 & 140 & 130 & 120 & 110 & 100 & 90 & $\begin{array}{c}80 \\
80\end{array}$ & 70 & 60 & 50 & 40 & 30 & 20 & $10_{145}$ & 0 & -1 \\
\hline
\end{tabular}




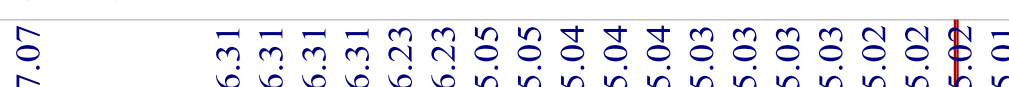

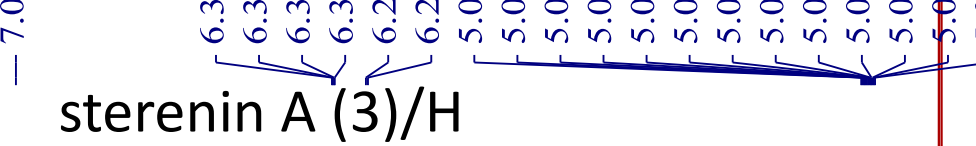

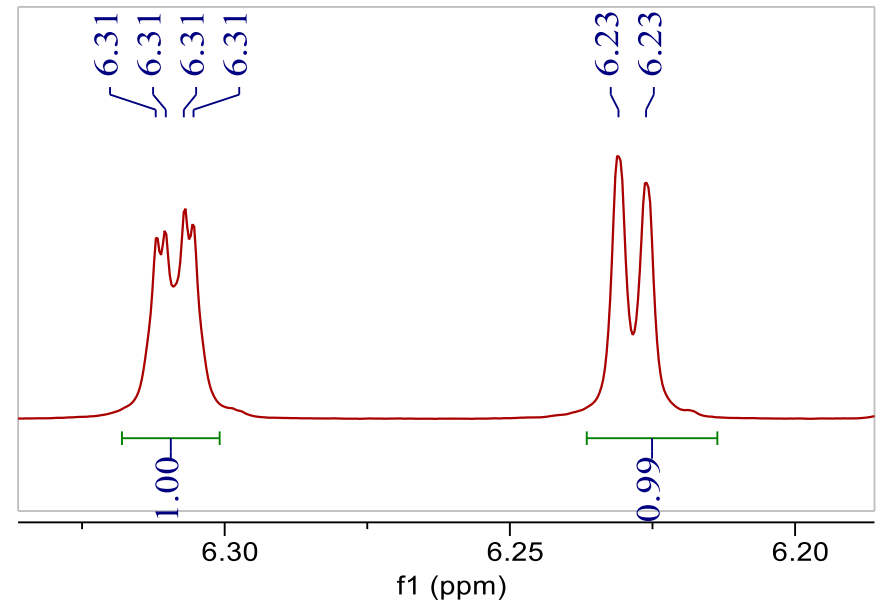

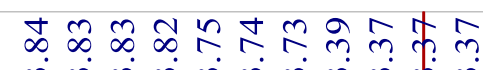

in

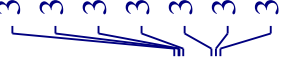

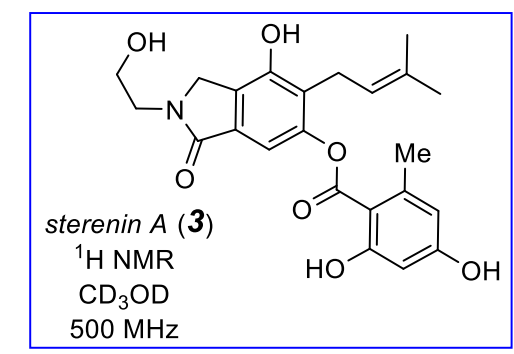
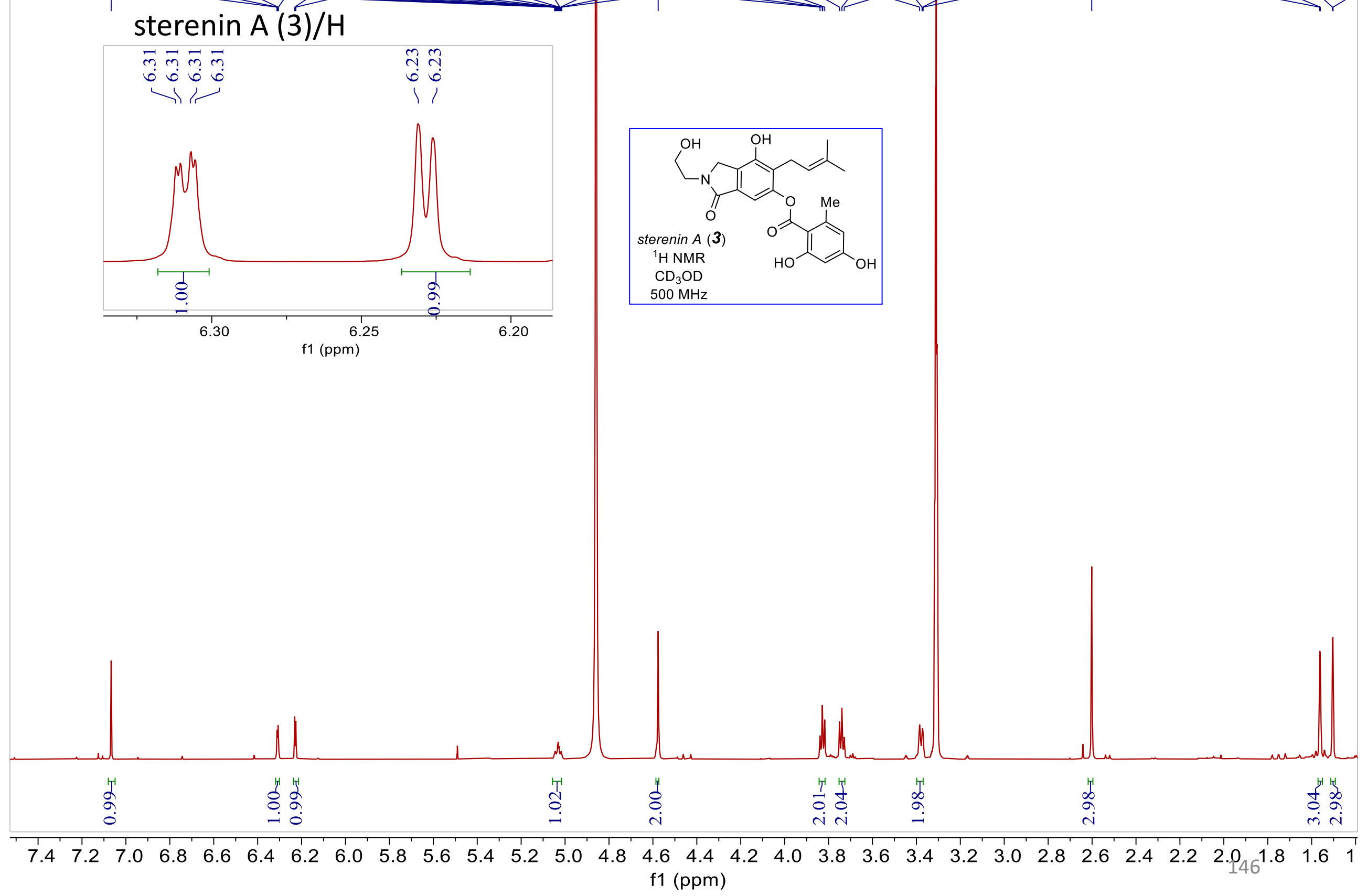
Znu, Zhang, \& Hoye

정ㅇㅇ

도웡

군

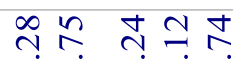

$\circ+\infty$ Supporting Information

$S 139$ of $S 139$

$\infty \approx \hat{\imath})$

ป்ं sterenin $A(3) / C$
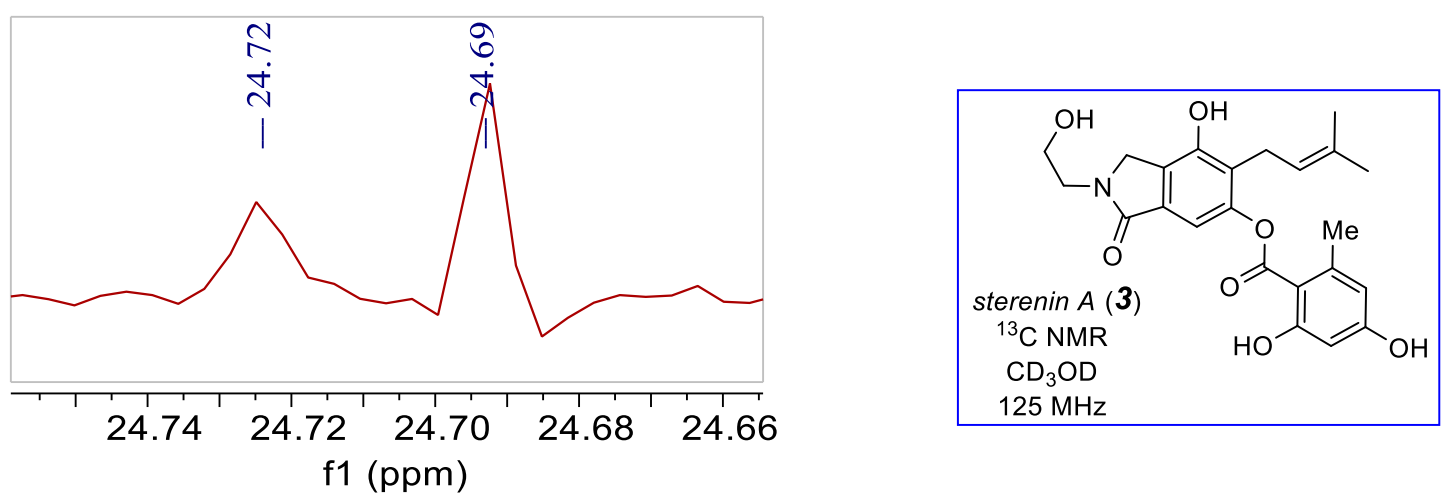

f1 (ppm)
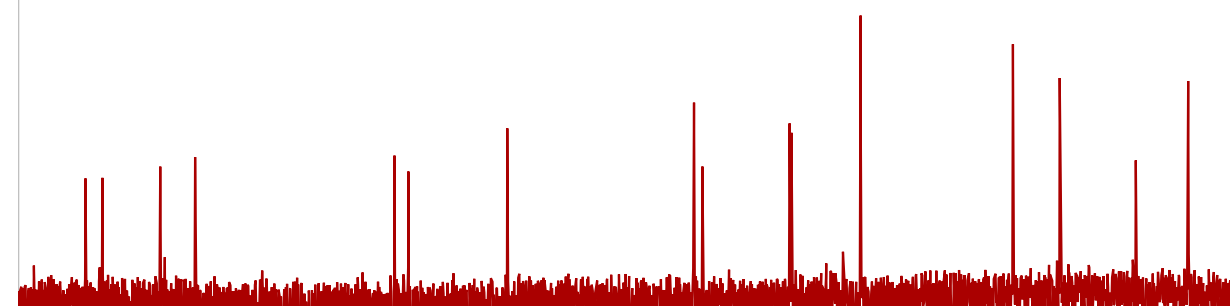